\title{
Ovarian cancer cachexia
}

Citation for published version (APA):

Ubachs, J. (2021). Ovarian cancer cachexia: a focus on body composition. [Doctoral Thesis, Maastricht University]. Gildeprint Drukkerijen. https://doi.org/10.26481/dis.20211217ju

Document status and date:

Published: 01/01/2021

DOI:

10.26481/dis.20211217ju

Document Version:

Publisher's PDF, also known as Version of record

\section{Please check the document version of this publication:}

- A submitted manuscript is the version of the article upon submission and before peer-review. There can be important differences between the submitted version and the official published version of record.

People interested in the research are advised to contact the author for the final version of the publication, or visit the DOI to the publisher's website.

- The final author version and the galley proof are versions of the publication after peer review.

- The final published version features the final layout of the paper including the volume, issue and page numbers.

Link to publication

\footnotetext{
General rights rights.

- You may freely distribute the URL identifying the publication in the public portal. please follow below link for the End User Agreement:

www.umlib.nl/taverne-license

Take down policy

If you believe that this document breaches copyright please contact us at:

repository@maastrichtuniversity.nl

providing details and we will investigate your claim.
}

Copyright and moral rights for the publications made accessible in the public portal are retained by the authors and/or other copyright owners and it is a condition of accessing publications that users recognise and abide by the legal requirements associated with these

- Users may download and print one copy of any publication from the public portal for the purpose of private study or research.

- You may not further distribute the material or use it for any profit-making activity or commercial gain

If the publication is distributed under the terms of Article $25 \mathrm{fa}$ of the Dutch Copyright Act, indicated by the "Taverne" license above, 


\section{Ovarian}

cancer

cachexia

a focus on body composition

J. Ubachs 



\section{Ovarian cancer cachexia}

\section{A focus on body composition}

Jorne Ubachs 
C Copyright Jorne Ubachs, Maastricht 2021

ISBN: 978-94-6419-353-4

Ontwerp: Tom Kölker; tomkolker.nl

Printing: Gildeprint

All rights reserved. No part of this publication may be reproduced, distributed, or transmitted in any form or by any means, including photocopying, recording, or other electronic or other mechanical methods, without the prior written permission from the author. 


\title{
Ovarian cancer cachexia
}

\author{
A focus on body composition
}

\section{PROEFSCHRIFT}

ter verkrijging van de graad van doctor aan de Universiteit Maastricht, op gezag van de Rector Magnificus, Prof. dr. Rianne M. Letschert volgens het besluit van het College van Decanen, in het openbaar te verdedigen op vrijdag 17 december 2021 om 10.00 uur

door

Jorne Ubachs 


\section{Promotores}

Prof. dr. R.F.P.M. Kruitwagen

Prof. dr. S.W.M. Olde Damink

Prof. dr. T. Van Gorp

\section{Copromotor}

Dr. S.S. Rensen

\section{Beoordelingscommissie}

Prof. dr. J. Wildberger (voorzitter)

Prof. dr. C. Dejong

Prof. dr. K. Van de Vijver (Universiteit Gent, België)

Prof. dr. H. Nijman (Universitair Medisch Centrum Groningen)

Dr. R. Lalisang 


\section{Table of contents}

Chapter 1 General introduction

Chapter 2 Sarcopenia and ovarian cancer survival: A systematic review and meta-analysis

Chapter 3 Psoas muscle area is not representative of total skeletal muscle area in the assessment of sarcopenia in ovarian cancer

Chapter 4 The influence of sarcopenia on survival and surgical complications in ovarian cancer patients undergoing primary debulking surgery

Chapter 5 No influence of sarcopenia on survival of ovarian cancer patients in a prospective validation study

Chapter 6 Ovarian cancer ascites induces skeletal muscle wasting in vitro and reflects sarcopenia in patients

Chapter 7 General Discussion

Chapter 8 Summary

Chapter 9 Impact Paragraph

Chapter 10 Epilogue

Dankwoord

About the author

List of publications

List of abbreviations 

General introduction 


\section{Introduction}

Ovarian cancer is the most lethal gynecological malignancy, claiming 185.000 lives annually.[1] Around 1300 women in the Netherlands are diagnosed with ovarian cancer each year and the annual death rate is approximately 1000.[2] Early stage ovarian cancer can go undetected for a long period of time since symptoms like abdominal distension, bloating, nausea, fatigue, altered micturition, and obstipation [3] do not always develop before disease has progressed to an advanced stage. Consequently, the poor survival rates of patients with ovarian cancer can be largely attributed to the fact that the majority (70\%) of newly diagnosed patients present themselves with advanced stage disease (FIGO (International Federation of Gynecology and Obstetrics) stage IIB-IVB). The five-year survival rates of early stage (FIGO IIIA) and advanced stage ovarian cancer are $75-100 \%$ versus $20-60 \%$, respectively.[4] In case of advanced stage ovarian cancer, optimal treatment currently consists of primary cytoreductive surgery followed by adjuvant chemotherapy, usually consisting of a combination of taxane and platinum based chemotherapy. If primary cytoreductive surgery is considered not feasible, neoadjuvant chemotherapy is given to reduce tumor load and interval cytoreductive surgery is performed after 3 cycles of chemotherapy.[5] Bevacizumab, a humanized monoclonal antibody aimed at vascular endothelial growth factor, was added to standard upfront therapy with the rationale to increase tumor perfusion and increase chemosensitivity when given alongside cytotoxic agents.[6] Over the last few years, studies with oral poly ADP ribose polymerase (PARP) inhibitors have been successful. PARP-inhibitors can prevent repair of single-stranded DNA damage, leading to breaks in double-stranded DNA after replication. These double stranded DNA breaks cannot be repaired in cells with deficiencies in the homologous repair pathway, which is encountered in BRCA mutated tumors. This leads to increased cell death in tumors.[7] PARP inhibitors are given after a response to platinum based treatment. One of the most recent developments in the treatment of epithelial ovarian cancer was the addition of intraperitoneal hyperthermic chemotherapy during interval cytoreductive surgery, which prolonged both recurrence free survival and overall survival in patients with stage III epithelial ovarian cancer. [8] Despite these recent developments leading to improvements in 5-year overall survival, they reflect a more prolonged disease control rather than a better chance of cure.[9]

\section{Definition of cancer cachexia}

There is an ongoing quest for new therapeutic targets for cancer treatment. In this context, recently, the metabolic phenotype of the host has gained considerable attention. Already in 1982, an 'adverse metabolic phenotype' was shown to be associated with worse outcome in cancer patients.[10] This adverse metabolic phenotype is also called 'cancer cachexia'. Cancer patients who have experienced weight loss of $>5 \%$ over the past 6 months are considered cachectic. Patients are also considered cachectic if weight loss $>2 \%$ was experienced in combination with a $\mathrm{BMI}$ of $<20$ or in combination with a low appendicular skeletal muscle index (SMI) consistent with sarcopenia.[11] Body weight loss is usually one of the most prominent symptoms of cancer cachexia. It results from a negative protein and energy balance driven by abnormal metabolism and/or reduced food intake.[11] Diagnosing cachexia in ovarian cancer patients can be challenging since weight loss is often masked by accumulation of ascites. Therefore, in ovarian cancer patients it might be more useful to assess skeletal 
muscle quantity and quality (myosteatosis) instead of weight loss. Sarcopenia can be identified by objectifying muscle quantity and quality with routinely used imaging techniques.

\section{Challenges and symptoms of cachexia}

Next to the masking of body weight loss by ascites, assessing body weight loss can also be challenging since it is usually patient reported and therefore subject to body image perception. Men tend to overestimate their length whereas women tend to underestimate their body weight. Subjects satisfied with their body weight have been found to be more accurate in reporting BMI.[12] Alterations in body weight due to cancer cachexia also lead to the undermining of identity and self-esteem.[13] In addition, very little is known about the actual habitual dietary patterns of cancer patients, and the relationship between dietary intake, physical activity, and cachexia. Anorexia in cachectic cancer patients has both a psychological and somatic origin, but can also lead to psychosocial (anorexia, depression, psychological distress) effects. [11, 13, 14] Patients can experience distress about the inability to eat or feel pressured or guilty with regard to food intake and weight loss.[15] Often patients also report chemosensory disturbances to food (taste and smell) and experience early satiety and nausea due to decreased upper gastrointestinal tract motility. Furthermore, central regulation of appetite is altered and several hormones (ghrelin, leptin) and neuropeptides (NPY) are implicated in suppression of nutritional intake and maintaining high metabolic demand.[16]

Developing a detailed and accurate understanding of patient activity, food intake, and weight loss is paramount as this understanding may help to supply a blueprint for the development of an effective treatment algorithm for cancer-induced cachexia. By using novel objective measurement methods (integrated memory weight scales, wrist worn accelerometers and app-based platforms for nutrient intake), bias and errors associated with patient selfreporting can be eliminated.

\section{Body composition measurements}

Since a decreased skeletal muscle mass is characteristic of cancer cachexia, assessment of body composition is important. Dual X-ray absorptiometry (DEXA), bio-impedance analysis $(\mathrm{BIA})$, magnetic resonance imaging (MRI), and computed tomography (CT) are available methods to perform body composition measurements. MRI- and CT-scan-analysis are considered the gold standards for the measurement of skeletal muscle mass.[17-19] Sarcopenia is often defined by the skeletal muscle index (SMI), which is the total muscle area as observed on a CT-scan corrected for height. Since ovarian cancer patients undergo CT-scan imaging for routine diagnostic purposes, it has become the most frequently used method for quantifying skeletal muscle mass and quality in this population. Importantly, skeletal muscle quantity and quality, as assessed by CT-scan analysis, have been shown to be prognostic independent of body mass index. For example, in a study of 1473 patients with gastrointestinal or lung cancer with varying degrees of weight loss, different SMI, and myosteatosis, patients who presented with all three of these poor prognostic variables survived 8.4 months $(95 \% \mathrm{Cl}, 6.5-10.3)$. This was observed regardless of whether they were obese, overweight, normal weight, or underweight, and in sharp contrast to patients who had none of these features, who survived 28.4 months (95\% Cl, 24.2-32.6; $\mathrm{P}<0.001)$.[20] 
Mourtzakis et al. included cancer patients $(n=50)$ and compared fat mass and fat free mass in these patients with bioelectrical-impedance analysis (BIA), dual x-ray absorptiometry (DEXA), and CT-scan.[21] BIA over- or underestimated fat free mass substantially when compared with DEXA and changes over time were beyond the limit of detection for BIA. In contrast, CT-scan imaging at the $3^{\text {rd }}$ lumbar level (L3) strongly predicted total body fat mass and fat free mass.[21] Assessment of total skeletal muscle area at L3 on CT-scan images using SliceOmatic software (Tomovision, Montreal, QC, Canada) can be time consuming. Total skeletal muscle area at L3 consists of the muscles of the abdominal wall (rectus abdominis, external oblique, internal oblique, transverse abdominal), the psoas muscle, and the paraspinal musculature. Efforts have been made to identify single muscle (e.g. psoas) groups whose area would predict overall survival.[22] This would eliminate the need for assessment of total skeletal muscle area and therefore be easier and less time consuming. However, it has never been demonstrated that these muscles are associated with total body skeletal muscle mass.[21] Furthermore, by assessing a smaller area, the measurement error increases and single muscles might be subject to atrophy related to muscle specific functional impairments.

Skeletal muscle radiation attenuation (SMRA) is defined by the mean Hounsfield unit (HU)value of the skeletal muscle and has been used as a measure of muscle quality or myosteatosis. Skeletal muscle radiation attenuation is determined by calculating the mean $\mathrm{HU}$ over the total skeletal muscle area at L3. The HU-value is a quantitative scale for describing radiodensity and is used in CT-scanning. The physical density of the tissue is proportional to the absorption or attenuation of the X-ray beam. Water is arbitrarily defined to be $0 \mathrm{HU}$ and air as $-1000 \mathrm{HU}$. Denser tissue has more $X$-ray absorption and appears brighter; it has positive $\mathrm{HU}$ values. Less dense tissue has less $\mathrm{X}$-ray beam absorption as reflected by negative $\mathrm{HU}$ values, and appears darker.[23] Accumulation of fat in skeletal muscle is called myosteatosis; it is characterized by lower mean HU values since the density of skeletal muscle with fat infiltration is lower than that of skeletal muscle without infiltration of fat.[24] Although SMI is not greatly affected by the contrast phase of the CT scan, SMRA is majorly influenced by the contrast phase and quality of the CT scan. $[25,26]$ A difference of at least $8 \mathrm{HU}$ in mean SMRA was detected between the venous-portal and arterial phase.[25] Unfortunately, there are no universal or standardized cut-offs for the determination of sarcopenia or myosteatosis based on CT-scan imaging. Frequently encountered cut-offs are based on means, medians, quartiles, tertiles, standard deviations, or optimum stratification analyses. All of these seem to be more unbiased approaches towards determining a cut-off in comparison to using previously published cut-offs, which never exactly apply to other cohorts. However, this approach leads to a myriad of cut-off values being used in different studies, causing variation in the reported prevalence of sarcopenia and myosteatosis.

\section{Sarcopenia in ovarian cancer}

Sarcopenia is frequently encountered in the elderly but also in patients with heart failure, chronic renal failure, malnutrition, chronic obstructive pulmonary disease, and cancer. $[17,18]$ A decrease in skeletal muscle mass during neoadjuvant chemotherapy in patients with epithelial ovarian cancer has been reported to be associated with a significant survival disadvantage. In a recent study of 123 patients with high stage ovarian cancer scheduled for neoadjuvant chemotherapy, median overall survival for patients who lost skeletal muscle tissue was $916 \pm 99$ days, which was significantly shorter than survival of patients who 
maintained or gained skeletal muscle mass (1431 \pm 470 days).[27] SMI and SMRA have been quantified in several additional cohorts of ovarian cancer patients, with the purpose of identifying a potential association between baseline skeletal muscle mass and/or quality and survival.[22, 27-35] All of these studies assessed the association between skeletal muscle mass and outcome in ovarian cancer patients. Several were able to establish an association with overall survival [27, 28, 30,31] but some were not.[28, 29, 35] The different results in the different studies can be attributed to a difference in prognostic variables, such as disease stage, completeness of cytoreductive surgery, and performance score. All in all, this underscores the need for studies with large cohorts of patients across different disease stages, performance indices, and surgery outcomes. This is the only way in which we can discern the effects of the different variables on survival or complications. Of note, dose limiting toxicities are more common in ovarian cancer patients with low muscle mass, $[36,37]$ and sarcopenia is thought to affect the volume of distribution, protein binding, clearance, and metabolism of drugs.[38]

\section{Drivers of cachexia}

An increase in circulating systemic catabolic factors (e.g. activin-A, myostatin, and interferony) has been detected in several models of cancer cachexia.[39, 40] Activin-A is a member of the transforming growth factor $\beta$ superfamily and is produced by a number of cell types; it has a broad range of biological effects.[41] Most of its biological actions are exerted through the activin type II receptor $b$, which it shares with myostatin, a potent negative regulator of muscle mass. It is thought that activin-A leads to skeletal muscle atrophy through the use of the same receptor.[39] Autophagy has also been identified as a promotor of skeletal muscle atrophy and proteases have been proposed to initiate protein degradation in skeletal muscle during cancer cachexia. $[42,43]$ Alongside increased inflammation and circulating catabolic factors, a decrease in the circulating levels of the anabolic insulin-like growth factor 1 and development of insulin resistance was detected in a mouse model of cancer cachexia.[44]

Systemic inflammation as a response to tumor-derived factors is considered to be one of the main drivers of cancer cachexia. Consistent associations between high systemic CRP levels and body weight loss in cancer patients have been established.[45] Recent publications have provided considerable evidence for the mechanistic importance of links between muscle wasting and fat depletion, and tumor derived cytokines such as tumor necrosis factor- $\alpha$ (TNF$\alpha$ ), interleukin-1 (IL-1), and interleukin-6 (IL-6)[46, 47] For example, TNF- $\alpha$ can directly induce transcriptomic activity of nuclear factor-KB which promotes expression of genes involved in ubiquitin proteasome mediated breakdown of skeletal muscle cell proteins.[48-50] Interestingly, cytokine expression profiling of ascites from ovarian cancer patients revealed high levels of IL-6, IL-8, and MCP-1[51], factors that have been suggested to promote tissue wasting in individuals with cachexia.[52] Furthermore, the concentration of cachexia-related inflammatory cytokines in ovarian cancer ascites has been shown to be significantly higher in comparison to the serum concentrations of the same patient.[53] Since ovarian cancer ascites is relatively easily accessible, generally present in large quantities, and contains a high concentration of tumor-derived compounds, it represents an attractive experimental tool to study the impact of ovarian cancer-derived factors on skeletal muscle physiology. Although skeletal muscle metabolism and homeostasis is complex and many factors have been 
proposed to influence it, there are surprisingly little data on functional measurements of protein synthesis and amino acid incorporation in skeletal muscle in cancer cachexia.[54]

\section{Objectives of this thesis}

The overall aims of this thesis are to thoroughly investigate the association between sarcopenia, myosteatosis, and outcome in ovarian cancer patients, to optimize assessment of radiological images for body composition analyses, to get insight into skeletal muscle homeostasis in ovarian cancer related cachexia, and to develop objective methods for the assessment of cachexia.

\section{Objectives}

- To summarize and incorporate all available data on cachexia in ovarian cancer and to investigate the association between adverse body composition and outcome in ovarian cancer patients (chapter 2 ).

- To investigate if psoas muscle area correlates with total skeletal muscle area and if it can be used for survival analysis in ovarian cancer patients (chapter 3 ).

- To investigate if pre-treatment skeletal muscle index is associated with the development of surgical complications and/or overall survival in ovarian cancer patients undergoing primary cytoreductive surgery (chapter 4).

- To validate the previously published finding that a decreasing skeletal muscle mass during neoadjuvant chemotherapy is associated with worse overall survival in a study of a homogeneous cohort of ovarian cancer patients (chapter 5).

- To investigate if ovarian cancer ascites can induce changes in skeletal muscle protein metabolism in an in-vitro model and if these changes are associated with host metabolic phenotype (chapter 6). 


\section{References}

1. Ferlay, J., et al., Estimating the global cancer incidence and mortality in 2018: GLOBOCAN sources and methods. Int J Cancer, 2019. 144(8): p. 1941-1953.

2. Nederlandse Kanker Registratie-cijfers / IKNL; bijgewerkt tot 21-10-2019

3. Ebell, M.H., M.B. Culp, and T.J. Radke, A Systematic Review of Symptoms for the Diagnosis of Ovarian Cancer. Am J Prev Med, 2016. 50(3): p. 384-394.

4. Torre, L.A., et al., Ovarian cancer statistics, 2018. CA Cancer J Clin, 2018. 68(4): p. 284-296.

5. Vergote, I., et al., Neoadjuvant chemotherapy or primary surgery in stage IIIC or IV ovarian cancer. N Engl J Med, 2010. 363(10): p. 943-53.

6. Burger, R.A., et al., Incorporation of bevacizumab in the primary treatment of ovarian cancer. N Engl J Med, 2011. 365(26): p. 2473-83.

7. Ledermann, J.A., Extending the scope of PARP inhibitors in ovarian cancer. Lancet Oncol, 2019. 20(4): p. 470-472.

8. van Driel, W.J., S.N. Koole, and G.S. Sonke, Hyperthermic Intraperitoneal Chemotherapy in Ovarian Cancer. N Engl J Med, 2018. 378(14): p. 1363-1364.

9. Timmermans, M., et al., No improvement in long-term survival for epithelial ovarian cancer patients: A population-based study between 1989 and 2014 in the Netherlands. Eur J Cancer, 2018. 88: p. 31-37.

10.DeWys, W.D., Pathophysiology of cancer cachexia: current understanding and areas for future research. Cancer Res, 1982. 42(2 Suppl): p. 721s-726s.

11.Fearon, K., et al., Definition and classification of cancer cachexia: an international consensus. Lancet Oncol, 2011. 12(5): p. 489-95.

12.Ng, C.D., Errors in body mass index from self-reported data by sex and across waves of Add Health. Ann Epidemiol, 2019.

13.Vaughan, V.C., P. Martin, and P.A. Lewandowski, Cancer cachexia: impact, mechanisms and emerging treatments. J Cachexia Sarcopenia Muscle, 2013. 4(2): p. 95-109.

14.Mijnarends, D.M., et al., Muscle, Health and Costs: A Glance at their Relationship. J Nutr Health Aging, 2018. 22(7): p. 766-773.

15. Hopkinson, J.B., Psychosocial impact of cancer cachexia. J Cachexia Sarcopenia Muscle, 2014. 5(2): p. 89-94.

16.Laviano, A., et al., Neurochemical mechanisms for cancer anorexia. Nutrition, 2002. 18(1): p. 1005.

17.Cruz-Jentoft, A.J., et al., Sarcopenia: European consensus on definition and diagnosis: Report of the European Working Group on Sarcopenia in Older People. Age Ageing, 2010. 39(4): p. 412-23.

18. Muscaritoli, M., et al., Consensus definition of sarcopenia, cachexia and pre-cachexia: joint document elaborated by Special Interest Groups (SIG) "cachexia-anorexia in chronic wasting diseases" and "nutrition in geriatrics". Clin Nutr, 2010. 29(2): p. 154-9.

19.Cesari, M., et al., Biomarkers of sarcopenia in clinical trials-recommendations from the International Working Group on Sarcopenia. J Cachexia Sarcopenia Muscle, 2012. 3(3): p. 181-90.

20. Martin, L., et al., Cancer cachexia in the age of obesity: skeletal muscle depletion is a powerful prognostic factor, independent of body mass index. J Clin Oncol, 2013. 31(12): p. 1539-47.

21.Mourtzakis, M., et al., A practical and precise approach to quantification of body composition in cancer patients using computed tomography images acquired during routine care. Appl Physiol Nutr Metab, 2008. 33(5): p. 997-1006.

22.Conrad, L.B., et al., Pre-operative core muscle index in combination with hypoalbuminemia is associated with poor prognosis in advanced ovarian cancer. J Surg Oncol, 2018. 117(5): p. 10201028.

23.DenOtter, T.D. and J. Schubert, Hounsfield Unit, in StatPearls. 2019: Treasure Island (FL).

24.van Dijk, D.P., et al., Low skeletal muscle radiation attenuation and visceral adiposity are associated with overall survival and surgical site infections in patients with pancreatic cancer. J Cachexia Sarcopenia Muscle, 2017. 8(2): p. 317-326.

25.van Vugt, J.L.A., et al., Contrast-enhancement influences skeletal muscle density, but not skeletal muscle mass, measurements on computed tomography. Clin Nutr, 2018. 37(5): p. 1707-1714.

26. Rollins, K.E., et al., Body composition measurement using computed tomography: Does the phase of the scan matter? Nutrition, 2017. 41: p. 37-44. 
27.Rutten, I.J., et al., Loss of skeletal muscle during neoadjuvant chemotherapy is related to decreased survival in ovarian cancer patients. J Cachexia Sarcopenia Muscle, 2016. 7(4): p. 458-66.

28.Ataseven, B., et al., Skeletal Muscle Attenuation (Sarcopenia) Predicts Reduced Overall Survival in Patients with Advanced Epithelial Ovarian Cancer Undergoing Primary Debulking Surgery. Ann Surg Oncol, 2018. 25(11): p. 3372-3379.

29.Aust, S., et al., Skeletal Muscle Depletion and Markers for Cancer Cachexia Are Strong Prognostic Factors in Epithelial Ovarian Cancer. PLoS One, 2015. 10(10): p. e0140403.

30.Bronger, H., et al., Sarcopenia in Advanced Serous Ovarian Cancer. Int J Gynecol Cancer, 2017. 27(2): p. 223-232.

31.Rutten, I.J., et al., The influence of sarcopenia on survival and surgical complications in ovarian cancer patients undergoing primary debulking surgery. Eur J Surg Oncol, 2017. 43(4): p. 717-724.

32. Silva de Paula, N., et al., Sarcopenia and Skeletal Muscle Quality as Predictors of Postoperative Complication and Early Mortality in Gynecologic Cancer. Int J Gynecol Cancer, 2018. 28(2): p. 412 420.

33.Torres, M.L., et al., Nutritional status, CT body composition measures and survival in ovarian cancer. Gynecol Oncol, 2013. 129(3): p. 548-53.

34.Uccella, S., et al., Assessment of preoperative nutritional status using BIA-derived phase angle $(P h A)$ in patients with advanced ovarian cancer: Correlation with the extent of cytoreduction and complications. Gynecol Oncol, 2018. 149(2): p. 263-269.

35.Kumar, A., et al., Muscle composition measured by CT scan is a measurable predictor of overall survival in advanced ovarian cancer. Gynecol Oncol, 2016. 142(2): p. 311-6.

36.Prado, C.M., et al., The association between body composition and toxicities from the combination of Doxil and trabectedin in patients with advanced relapsed ovarian cancer. Appl Physiol Nutr Metab, 2014. 39(6): p. 693-8.

37. Yoshikawa, T., et al., Psoas muscle volume as a predictor of peripheral neurotoxicity induced by primary chemotherapy in ovarian cancers. Cancer Chemother Pharmacol, 2017. 80(3): p. 555-561.

38. Hopkins, J.J. and M.B. Sawyer, A review of body composition and pharmacokinetics in oncology. Expert Rev Clin Pharmacol, 2017. 10(9): p. 947-956.

39.Loumaye, A., et al., Role of Activin A and myostatin in human cancer cachexia. J Clin Endocrinol Metab, 2015. 100(5): p. 2030-8.

40.Acharyya, S., et al., Cancer cachexia is regulated by selective targeting of skeletal muscle gene products. J Clin Invest, 2004. 114(3): p. 370-8.

41.Xia, Y. and A.L. Schneyer, The biology of activin: recent advances in structure, regulation and function. J Endocrinol, 2009. 202(1): p. 1-12.

42.Tardif, N., et al., Autophagic-lysosomal pathway is the main proteolytic system modified in the skeletal muscle of esophageal cancer patients. Am J Clin Nutr, 2013. 98(6): p. 1485-92.

43. Costelli, P., et al., Ca(2+)-dependent proteolysis in muscle wasting. Int J Biochem Cell Biol, 2005. 37(10): p. 2134-46.

44. Asp, M.L., et al., Evidence for the contribution of insulin resistance to the development of cachexia in tumor-bearing mice. Int J Cancer, 2010. 126(3): p. 756-63.

45.Blum, D., et al., Cancer cachexia: a systematic literature review of items and domains associated with involuntary weight loss in cancer. Crit Rev Oncol Hematol, 2011. 80(1): p. 114-44.

46. Carson, J.A. and K.A. Baltgalvis, Interleukin 6 as a key regulator of muscle mass during cachexia. Exerc Sport Sci Rev, 2010. 38(4): p. 168-76.

47.Fearon, K.C., D.J. Glass, and D.C. Guttridge, Cancer cachexia: mediators, signaling, and metabolic pathways. Cell Metab, 2012. 16(2): p. 153-66.

48.Glass, D.J., Skeletal muscle hypertrophy and atrophy signaling pathways. Int J Biochem Cell Biol, 2005. 37(10): p. 1974-84.

49.Guttridge, D.C., et al., NF-kappaB-induced loss of MyoD messenger RNA: possible role in muscle decay and cachexia. Science, 2000. 289(5488): p. 2363-6.

50.Langen, R.C., et al., Inflammatory cytokines inhibit myogenic differentiation through activation of nuclear factor-kappaB. FASEB J, 2001. 15(7): p. 1169-80.

51.Matte, I., et al., Profiling of cytokines in human epithelial ovarian cancer ascites. Am J Cancer Res, 2012. 2(5): p. 566-80.

52.Talbert, E.E., et al., Circulating monocyte chemoattractant protein-1 (MCP-1) is associated with cachexia in treatment-naive pancreatic cancer patients. J Cachexia Sarcopenia Muscle, 2018. 9(2): p. 358-368. 
53.Penson, R.T., et al., Cytokines IL-1beta, IL-2, IL-6, IL-8, MCP-1, GM-CSF and TNFalpha in patients with epithelial ovarian cancer and their relationship to treatment with paclitaxel. Int J Gynecol Cancer, 2000. 10(1): p. 33-41.

54. Kamolrat, T. and S.R. Gray, The effect of eicosapentaenoic and docosahexaenoic acid on protein synthesis and breakdown in murine C2C12 myotubes. Biochem Biophys Res Commun, 2013. 432(4): p. 593-8. 


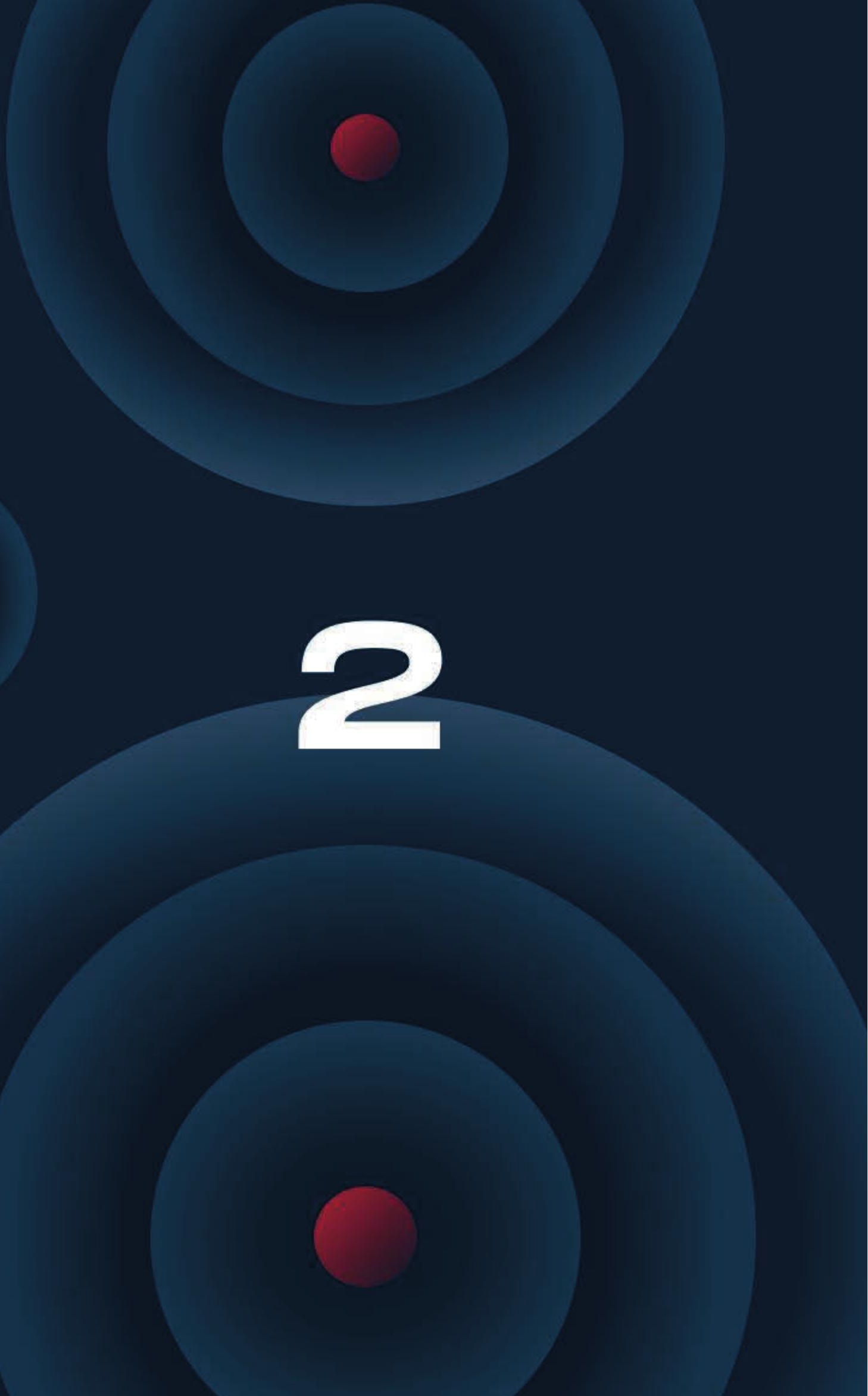




\section{Sarcopenia and ovarian cancer survival: A systematic review and meta-analysis}

Journal of cachexia, Sarcopenia and Muscle (2019)

Jorne Ubachs, Janine Ziemons, Iris Minis-Rutten, Roy Kruitwagen, Jos Kleijnen, Sandrina

Lambrechts, Steven Olde Damink, Sander Rensen, Toon Van Gorp 


\section{Abstract}

\section{Background}

Sarcopenia is the loss of skeletal muscle mass and function that occurs with advancing age and certain diseases. It is thought to have a negative impact on survival in cancer patients. Routine computed tomography imaging is often used to quantify skeletal muscle in cancer patients. Sarcopenia is defined by a low skeletal muscle index (SMI). Skeletal muscle radiation attenuation (SMRA) is used to define muscle quality. The primary aim of this meta-analysis was to study the association between sarcopenia or SMRA and overall survival (OS) or complications in patients with ovarian cancer.

\section{Methods}

Medline, Embase, CINAHL, and PEDro databases were searched from inception to 15 February 2019. Studies evaluating the prognostic effect of SMI and SMRA on ovarian cancer survival or surgical complications were included. Risk of bias and study quality were evaluated with the Quality in Prognosis Studies Instrument (QUIPS) according to the modified Grading of Recommendations Assessment, Development and Evaluation (GRADE) framework.

\section{Results}

The search strategy yielded 4,262 hits in all four databases combined. Ten and eight studies were included for qualitative and quantitative analysis, respectively. Meta-analysis revealed a significant association between the SMI and OS ( $p=0.007$; hazard ratio (HR): $1.11,95 \%$ confidence interval ( $\mathrm{Cl})$ : 1.03-1.20). SMRA was also significantly associated with OS ( $p<0.001$; HR: $1.14,95 \% \mathrm{Cl}: 1.08-1.20)$. Association between the SMI and surgical complications had borderline statistical significance ( $p=0.05$; HR: $1.23,95 \% \mathrm{Cl}: 1.00-1.52)$. The risk of bias assessed with QUIPS was high in all studies. The quality of the evidence was very low.

\section{Conclusion}

Whereas our meta-analysis indicated that a low SMI and Iow SMRA are associated with survival in ovarian cancer patients, the low quality of the source data precludes drawing definitive conclusions. 


\section{Introduction}

Despite intensified treatment and staging procedures, long-term survival (10-15 years) of women with epithelial ovarian cancer has not improved in the last 25 years. The observed improvements in 5-year overall survival (OS) reflect a more prolonged disease control rather than a better chance of cure.[1] Attempts to improve the management of ovarian cancer are focused on overcoming chemotherapy resistance, and intraperitoneal chemotherapy, immunotherapy, and personalized medicine.[2-5] With regards to personalized medicine, efforts are being made to explore new therapeutic targets, and the patients' physical ability to receive treatment is an important accompanying factor to be considered. This physical ability is partially reflected by the patients' muscle mass. Low muscle mass or sarcopenia has been identified as a prognostic factor in several malignancies including pancreatic[6], hepatic, biliary tract, gastrointestinal[7], and urothelial cancer.[8, 9]

Sarcopenia is frequently encountered in the elderly but also in patients with heart failure, chronic renal failure, malnutrition, chronic obstructive pulmonary disease, and cancer.[10, 11] Cancer-related sarcopenia is part of a syndrome called cancer cachexia, [12] which results from a negative protein and energy balance driven by abnormal metabolism and reduced food intake. Since the pathophysiology is not yet fully understood, therapeutic options remain limited.[13] The diagnosis of cancer cachexia is based on body weight loss alone or a combination of loss of body weight and muscle mass.[12] Diagnosing cachexia in ovarian cancer patients can be challenging since weight loss is often masked by accumulation of ascites. In ovarian cancer patients, it is therefore more reliable to quantify skeletal muscle instead of weight loss. Dual X-ray absorptiometry (DEXA), bio-impedance analysis (BIA), magnetic resonance imaging (MRI), and computed tomography (CT) are available methods to perform this quantification. MRI and CT-scanning are considered the gold standard for the quantification of skeletal muscle mass.[10, 14] Skeletal muscle mass, as assessed by CT-scan analysis, is a prognostic factor independent of body mass index. [15] Since ovarian cancer patients undergo CT-scan imaging for routine diagnostic purposes, it has become the most frequently used method for quantifying skeletal muscle mass and quality in this population. Sarcopenia is often defined by the skeletal muscle index (SMI), which is the total muscle area as observed on the CT-scan corrected for height. Skeletal muscle radiation attenuation (SMRA) is defined by the mean Hounsfield unit-value of the skeletal muscle and has been used as a measure of muscle quality. The Hounsfield unit-value is a quantitative scale for describing radiodensity and is used in CT-scanning.

SMI and SMRA have been quantified in several cohorts of ovarian cancer patients,[16-26] with the purpose of identifying a potential association between skeletal muscle mass or quality and survival. Assessment of body composition to identify cachexia is more reliable in ovarian cancer patients since body weight changes can be masked by accumulation of ascites. These assessments might elicit a potential modifiable prognostic factor in ovarian cancer. Since some studies which analyzed body composition report associations between SMI or SMRA and survival/complications whilst others do not, we feel there is a need to summarize the findings and come to a complete overview of all available literature on this subject. This meta-analysis is the first to provide a quantitative and qualitative assessment of SMI and SMRA related to ovarian cancer survival and development of complications. 


\section{Methods}

\section{Electronic search}

This analysis was conducted according to the Preferred Reporting Items for Systematic Reviews and Meta-Analyses (PRISMA).[27] An independent search of the Medline (via OvidSP), Embase (via OvidSP), CINAHL (via EBSCO), and PEDro databases was carried out. The search was completed on 15 February 2019. Reviews, case reports, opinion articles, conference abstracts, and non-published data were excluded.

\section{Study selection}

Studies that met the following criteria were included for quantitative meta-analysis: (1) studies of patients with ovarian cancer; (2) studies that associated SMI or SMRA with OS or complication rate; (3) skeletal muscle was quantified by CT-scan; (4) studies that reported hazard ratios (HR), odds ratios (OR) or event counts, and $95 \%$ confidence intervals $(\mathrm{Cl})$ for survival or complications. Studies reporting insufficient data for calculating HR, OR, and $95 \%$ $\mathrm{Cl}$ were excluded. Two researchers (JU and JZ) performed an independent search of the databases and selected abstracts. They were blinded to each other's results. Agreement upon selected abstracts was reached afterwards. Both researchers then independently screened full-text articles and came to agreement for inclusion after each made their individual selection. All databases were screened from inception up to 2019 (see Appendix 1 and 2 for full electronic search and used terms). In the case of duplicate studies or reports, the most recent version was included.

\section{Data extraction}

Data extraction was also performed independently by the two researchers. The following data were retrieved: first author name, year of publication, type of study, number of patients included, mean/median population age, disease stage, method of quantifying skeletal muscle mass, cut-off point for SMI or low SMRA, prevalence of SMI and low SMRA and associated HR or OR with $95 \% \mathrm{Cl}$ for OS, and associated HR or OR for complications. HRs and ORs were extracted from univariate and multivariate analyses, or calculated from event counts.

\section{Quality assessment}

Risk of bias for individual studies was assessed with the Quality in Prognosis Studies (QUIPS) Risk of Bias Assessment Instrument for Prognostic Factor Studies.[28, 29] The QUIPS tool has several domains: study participation, study attrition, prognostic factor measurement, outcome measurement, study confounding, and statistical analysis and reporting. All domains consist of several criteria of which the combined rating produces a classification of high, moderate, or low risk. All domains were scored by the two individual researchers. The overall risk of bias was considered low if $\leq 2$ domains were rated a moderate risk of bias and all others were rated a low risk of bias. The overall risk of bias was considered moderate if $>2$ domains were rated a moderate risk of bias and all others were rated a low risk of bias. The overall risk of bias was considered high if $\geq 1$ domain was rated a high risk of bias, irrespective of all other domains. Consensus was reached after classification by the individual researchers. The quality of the overall quantitative outcome of this meta-analysis was determined with the Grading of Recommendations Assessment, Development, and Evaluation (GRADE) tool, which objectively assesses outcomes based on different domains using a scoring system. Outcomes are allocated a score based on study design, which can be downgraded if certain criteria for quality are not met. This results in an objective score with a GRADE rating ranging from 1 (very 
low quality; little confidence in the estimate; the true prognosis is likely to be substantially different from the estimate) to 4 (high quality; very confident that the true prognosis lies close to that of the estimate).[30][31]

\section{Data handling and statistical analysis}

Meta-analyses were performed in Review Manager (RevMan v5.3, 2014; The Cochrane Collaboration, Copenhagen, Denmark) and results were displayed in forest plots. HRs for survival and ORs for surgical complications were either retrieved or calculated from the included studies. HRs and ORs were calculated with an indirect variance estimation as proposed by Parmar et.al.[32] A fixed effects model was used because effects were not expected to substantially vary between studies because the methodologies were highly homogeneous. Results were considered statistically significant at $p<0.05$. Statistical heterogeneity, or inconsistency, was assessed using the chi-squared $\left(X^{2}\right)$ and $I^{2}$ tests. An $1^{2} \geq 65 \%$ and a $p$-value for the $X^{2-}$ test below 0.10 was considered a violation of the assumption of homogeneity.

\section{Results}

\section{Search results / included studies}

The search of four databases yielded 4,262 hits. After the automatic removal of 66 duplicates, 4,196 titles and abstracts were screened by two independent researchers (JU and JZ). After screening abstracts and titles and reaching agreement, 38 full-text articles were considered for inclusion. After a full-text review of the 38 articles, ten remained for qualitative analysis. Out of these ten articles, eight remained for quantitative analysis. The selection process and reasons for exclusion are shown in Figure 1. A manual review of the references yielded no extra inclusions. All included studies were published from 2013 onwards. Study characteristics are summarized in Table 1.

\section{Included studies}

All eight included studies for quantitative analysis were of a retrospective nature. Six out of the eight included studies that evaluated SMI and its association with OS in ovarian cancer, comprising a total of 1,198 patients.[16-20, 22] Four studies evaluated SMRA and its association with OS in ovarian cancer, comprising a total of 975 patients. [17, 18, 20, 22] Three studies evaluated SMI and its association with short term post-operative morbidity, comprising a total of 407 patients. [17, 23, 24] All studies were published in international peerreviewed journals. Skeletal muscle mass was quantified on an axial CT-scan at the third or fourth lumbar level. The SMI or SMRA were calculated from total muscle area as determined from the lumbar CT-scan in all studies, except one that used the core muscle index (CMI; psoas muscle area) for quantification.[24] Two studies were not included for quantitative analyses: one that analyzed phase-angle with BIA instead of with CT [25], and the other did not determine a cut-off for defining sarcopenia but analyzed muscle mass as a continuous variable.[21] 


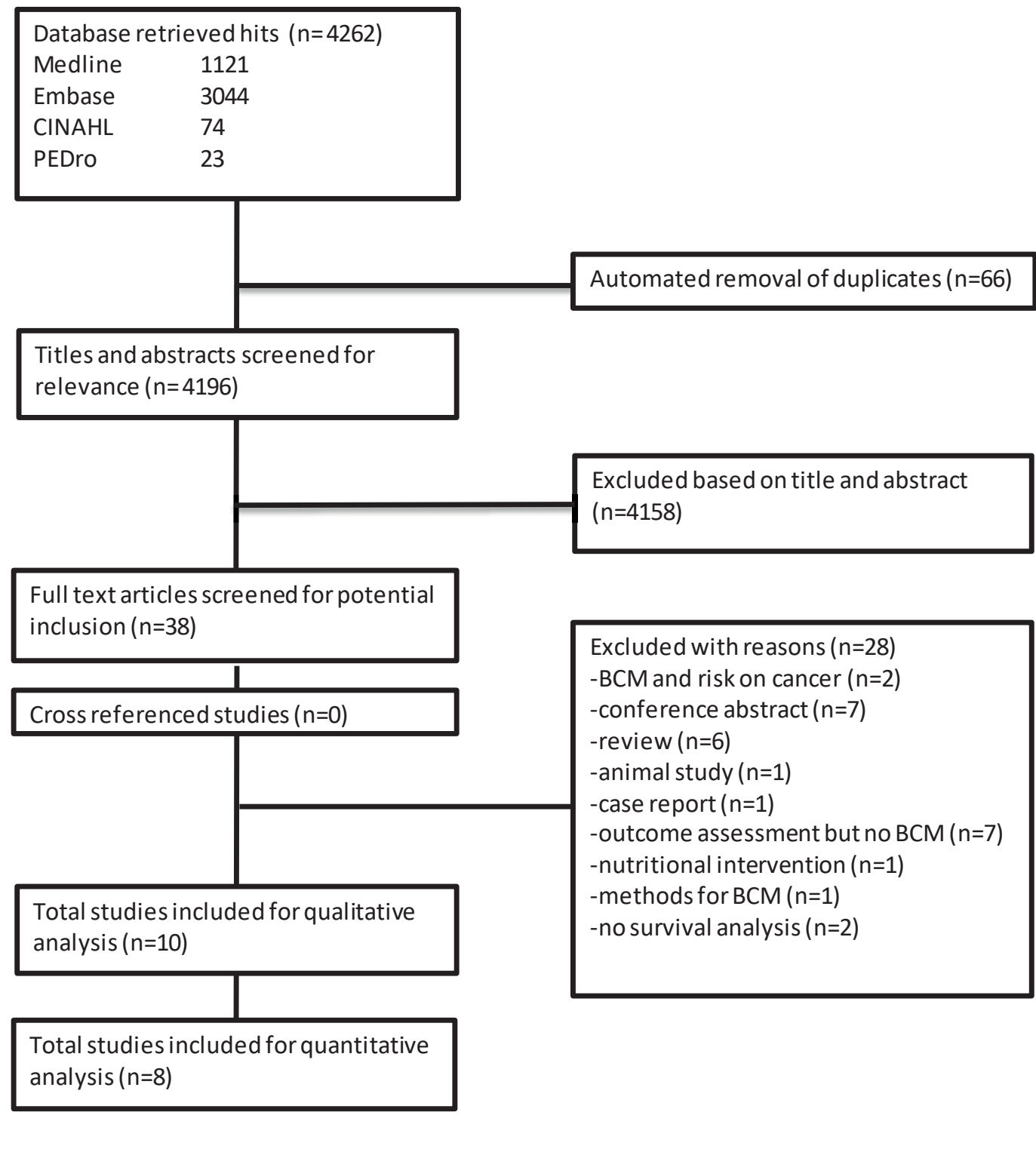

Figure 1 Flow diagram depicting the selection process for studies. BCM; body composition measurements

\section{Patient characteristics}

All patients underwent primary or interval debulking surgery combined with adjuvant or neoadjuvant chemotherapy. One study included patients with endometrial and ovarian cancer. For this meta-analysis, only the cohort of ovarian cancer patients was included. [23] FIGO (International Federation for Gynecologic Oncology) stage across all studies ranged from I-IV. 
The distribution of patients according to FIGO stage was as follows: I, $\mathrm{n}=106$ (6.8\%); II, n=55 (3.5\%); III, n=968 (62\%); IV, n=433 (27.7\%).

\section{SMI, SMRA, and OS}

The meta-analysis of the univariate data of the influence of the SMI on OS is depicted in Figure 2. The overall effect of sarcopenia on OS was significant ( $p=0.007$; HR: $1.11,95 \% \mathrm{Cl}: 1.03-$ 1.20). Statistical consistency between the compared hazard ratios and $95 \% \mathrm{Cls}$ was evaluated with the $\mathrm{X}^{2}$ - and $\mathrm{I}^{2}$-tests, which returned an $\mathrm{X}^{2} \mathrm{p}=0.15$ and an $\mathrm{I}^{2}$ of $38 \%$, indicating methodological homogeneity, or consistency, between studies. The fixed effects model was deliberately chosen because the methodologies for measuring SMI and its association with OS used in the studies were homogeneous; however, use of the random effects model would not have influenced the results ( $p=0.03$; HR: 1.12, 95\% Cl: 1.01-1.24). In meta-analysis of the multivariate data (fixed effects), there was also a significant association between SMI and OS ( $p=0.02$; HR: $1.17,95 \% \mathrm{Cl}: 1.03-1.33$ ) (Figure 3). An $\mathrm{X}^{2}$-test $p$-value of 0.31 and an $\mathrm{I}^{2}$ of $14 \%$ indicated consistency between studies for this association.

The meta-analysis of univariate data of the influence of low SMRA on OS is depicted in Figure 4. The overall effect of low SMRA on OS was significant ( $p<0.001 ; \mathrm{HR}: 1.14,95 \% \mathrm{Cl}: 1.08-1.20$ ). Again, the fixed effects model was chosen; however, a random effects model would not have influenced the results ( $p<0.001 ; H R: 1.15,95 \% \mathrm{Cl}: 1.08-1.22)$. An $X^{2}$-test $p$-value of 0.34 and an $\mathrm{I}^{2}$ of $11 \%$ indicated consistency. The meta-analysis of the multivariate data (fixed effects) revealed a significant association between SMRA and OS ( $p=0.001$; HR: 1.13, 95\% Cl: $1.06-$ 1.20) (Figure 5), while tests for consistency yielded an $X^{2}$ of $p=0.07$ and an $I^{2}$ of $62 \%$.

\section{Sarcopenia and surgical complications}

The meta-analysis of the effect of a low SMI on the development of surgical complications is depicted in Figure 6. The overall effect of low SMI vs. high SMI was borderline significant ( $p=0.05 ; \mathrm{HR}: 1.23,95 \% \mathrm{Cl}: 1.00-1.52)$. A fixed effects model was chosen, but using a random effects model would not have changed the outcome ( $p=0.10$; HR: $1.26,95 \% \mathrm{Cl}: 0.95-1.67)$. Studies showed statistical consistency $\left(X^{2} p=0.17 ; 1^{2} 43 \%\right)$.

\section{Risk of bias and GRADE assessment}

According to the QUIPS checklist, all included studies had an overall high risk of bias. An overview per domain can be found in Appendix 3. According to the GRADE rating, quality of the outcome was considered 'very low' for the reliability of all associations. The findings are summarized in Table 2. The reason for downgrading 'indirectness' was because of the use of different assessments of SMI in the surgical complications cohort (total SMI vs. CMI). Indirectness was defined as the correspondence of the population of interest with the population included for analysis. Imprecision was detected in the forest plot on the influence of SMI on OS. Imprecision was defined as an insufficient sample size of included studies and very wide confidence intervals in meta-analysis, crossing the null in $\geq 50 \%$ cases. 


\begin{tabular}{|c|c|c|c|c|c|c|c|c|c|}
\hline Author & Year & Country & Study design & $n$ & $\begin{array}{l}\text { Age } \\
*\end{array}$ & $\begin{array}{l}\text { Disease } \\
\text { stage } \\
\text { (FIGO) }\end{array}$ & $\begin{array}{l}\text { Measuremen } \\
\mathbf{t}\end{array}$ & $\begin{array}{l}\text { SMI cut- } \\
\text { point } \\
(\mathrm{cm} 2 / \mathrm{m} 2)\end{array}$ & $\begin{array}{l}\text { SMRA } \\
\text { (HU) } \\
\text { cut-point }\end{array}$ \\
\hline Torres & 2013 & USA & retrospective & 83 & 68,4 & IIIc-IV & $\begin{array}{l}\text { CT based 3rd } \\
\text { lumbar level }\end{array}$ & n.a. & n.a. \\
\hline Aust & 2015 & Austria & retrospective & 140 & 60 & I-IV & $\begin{array}{l}\text { CT based 3rd } \\
\text { lumbar level }\end{array}$ & 41 & 39 \\
\hline Rutten & 2016 & Netherlands & retrospective & 123 & 66,5 & II-IV & $\begin{array}{l}\text { CT based 3rd } \\
\text { lumbar level }\end{array}$ & 41.5 & n.a. \\
\hline Kumar & 2016 & USA & retrospective & 296 & 64,6 & IIIc-IV & $\begin{array}{l}\text { CT based 3rd } \\
\text { lumbar level }\end{array}$ & 39 & 36.4 \\
\hline Bronger & 2017 & Germany & retrospective & 105 & 65 & III-IV & $\begin{array}{l}\text { CT based 3rd } \\
\text { lumbar level }\end{array}$ & 38.5 & n.a. \\
\hline Rutten & 2017 & Netherlands & retrospective & 216 & 62,5 & II-IV & $\begin{array}{l}\text { CT based 3rd } \\
\text { lumbar level }\end{array}$ & 38.73 & 36 \\
\hline Conrad & 2018 & USA & retrospective & 102 & 55 & III-IV & $\begin{array}{l}\text { CT based } 4 \text { th } \\
\text { lumbar level }\end{array}$ & 2.8 (CMI) & n.a. \\
\hline $\begin{array}{l}\text { Paula de } \\
\text { Silva }\end{array}$ & 2018 & Brazil & retrospective & 89 & n.r. & I-IV & $\begin{array}{l}\text { CT based 3rd } \\
\text { lumbar level }\end{array}$ & 38.9 & quartiles \\
\hline Ataseven & 2018 & Germany & retrospective & 323 & 60 & IIIb-IVb & $\begin{array}{l}\text { CT based 3rd } \\
\text { lumbar level }\end{array}$ & $\begin{array}{l}38.5 \\
39 \\
41\end{array}$ & 32 \\
\hline Uccella & 2018 & Italy & retrospective & 70 & 58,5 & IIIC-IV & $\begin{array}{l}\text { Phase angle } \\
\text { (BIA) }\end{array}$ & n.a. & n.a. \\
\hline
\end{tabular}




\begin{tabular}{|c|c|c|c|c|}
\hline $\begin{array}{l}\text { Prevalence of } \\
\text { sarcopenia \% } \\
\text { (based on } \\
\text { SMI) }\end{array}$ & $\begin{array}{l}\text { Qualitative/ } \\
\text { quantitative }\end{array}$ & $\begin{array}{l}\text { Quips } \\
\text { score }\end{array}$ & $\begin{array}{l}\text { Outcome } \\
\text { (OS/complications) }\end{array}$ & Main outcome \\
\hline n.a. & Qualitative & high & OS & $\begin{array}{l}\text { Total muscle mass not predictive for survival. } \\
\text { Lower subcutaneous fat and intramuscular fat } \\
\text { associated with poor OS and longer hospital } \\
\text { stay. }\end{array}$ \\
\hline 28.9 & Quantitative & high & OS & $\begin{array}{l}\text { Low SMRA is associated with poor OS, SMI is not } \\
\text { associated with poor OS }\end{array}$ \\
\hline 50.4 & Quantitative & high & OS & $\begin{array}{l}\text { Longitudinal loss of SMI is associated with poor } \\
\text { OS, low baseline SMI is not associated with poor } \\
\text { OS }\end{array}$ \\
\hline 39 & Quantitative & high & OS & $\begin{array}{l}\text { Low SMRA is associated with poor OS, SMI is not } \\
\text { associated with poor OS }\end{array}$ \\
\hline 11 & Quantitative & high & OS & Low SMI is associated with poor OS \\
\hline 32 & Quantitative & high & both & $\begin{array}{l}\text { Low SMI is not associated with poor OS or } \\
\text { surgical complications. Low SMRA not associated } \\
\text { with OS }\end{array}$ \\
\hline 54 & Quantitative & high & both & $\begin{array}{l}\text { Low SMI combined with hypoalbuminemia is } \\
\text { associated with worse OS. Low SMI not } \\
\text { associated with complications }\end{array}$ \\
\hline 25.8 & Quantitative & high & $\begin{array}{l}30 \text { day mortality } \\
\text { and complications }\end{array}$ & $\begin{array}{l}\text { Low SMI and skeletal muscle quality associated } \\
\text { with development of surgical complications }\end{array}$ \\
\hline $\begin{array}{l}29.4 \\
33.7 \\
47.1\end{array}$ & Quantitative & high & OS & $\begin{array}{l}\text { Low SMRA associated with OS, low SMI not } \\
\text { associated with OS }\end{array}$ \\
\hline n.a. & Qualitative & high & complications & $\begin{array}{l}\text { Low phase angle associated with surgical } \\
\text { complications }\end{array}$ \\
\hline
\end{tabular}

Table 1 Study characteristics and main findings. n; no. of included patients, FIGO; international federation for gynecologic oncology, SMI; skeletal muscle index, SMRA; skeletal muscle radiation attenuation, $\mathrm{HU}$; Hounsfield units, OS; overall survival, BIA; bio-electrical impedance analysis, n.r.; not reported, n.a.; not applicable *mean or median as reported 


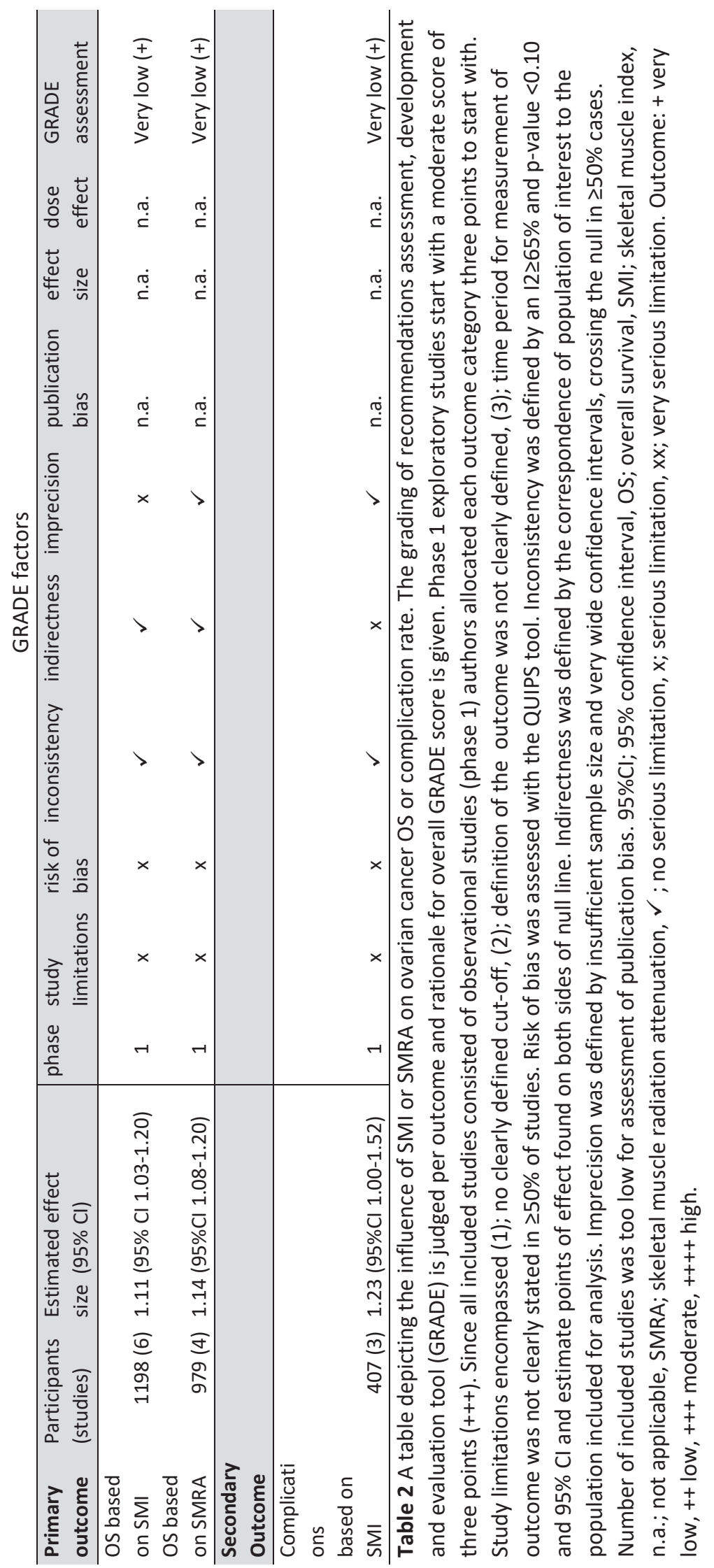




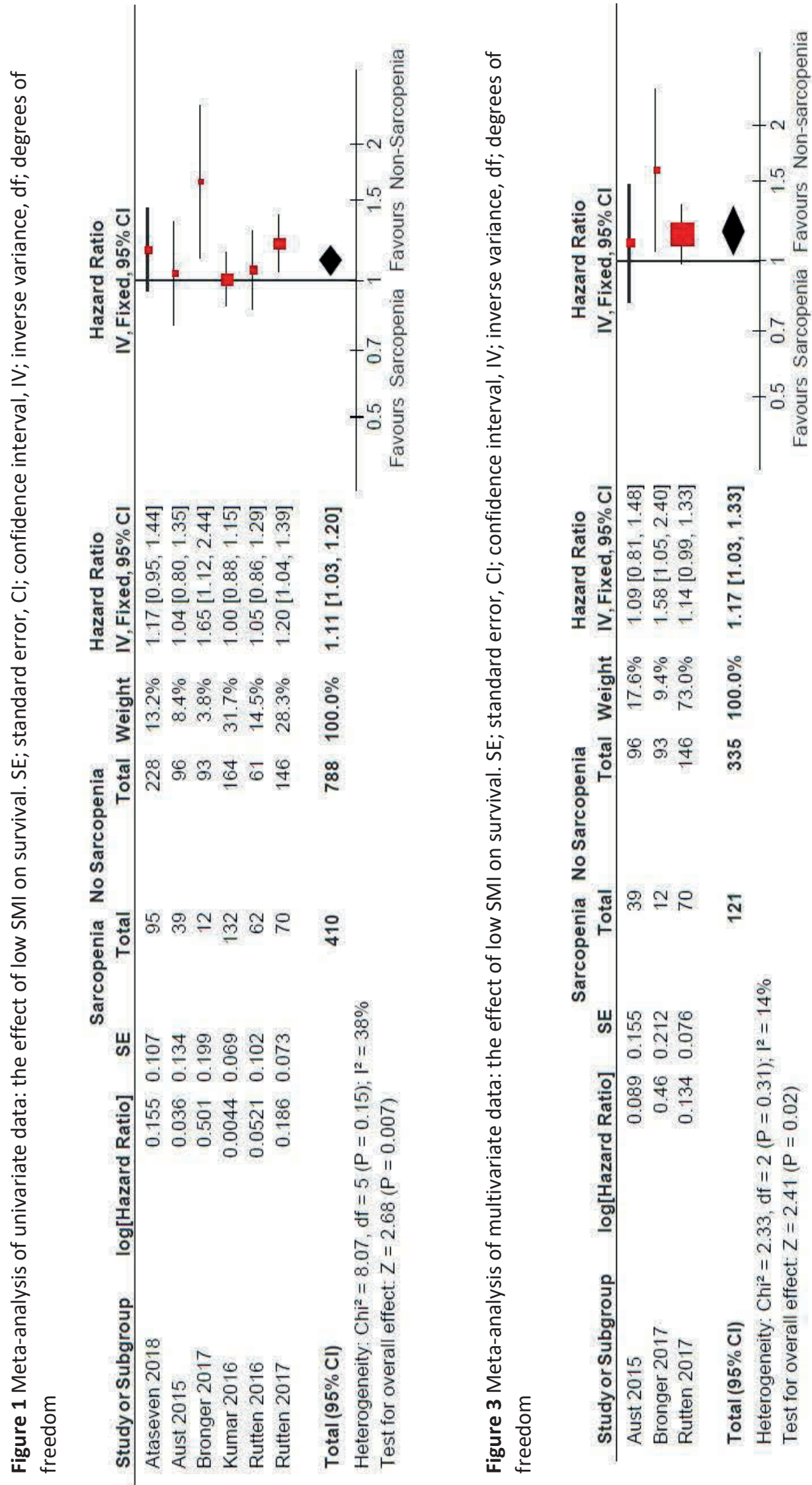




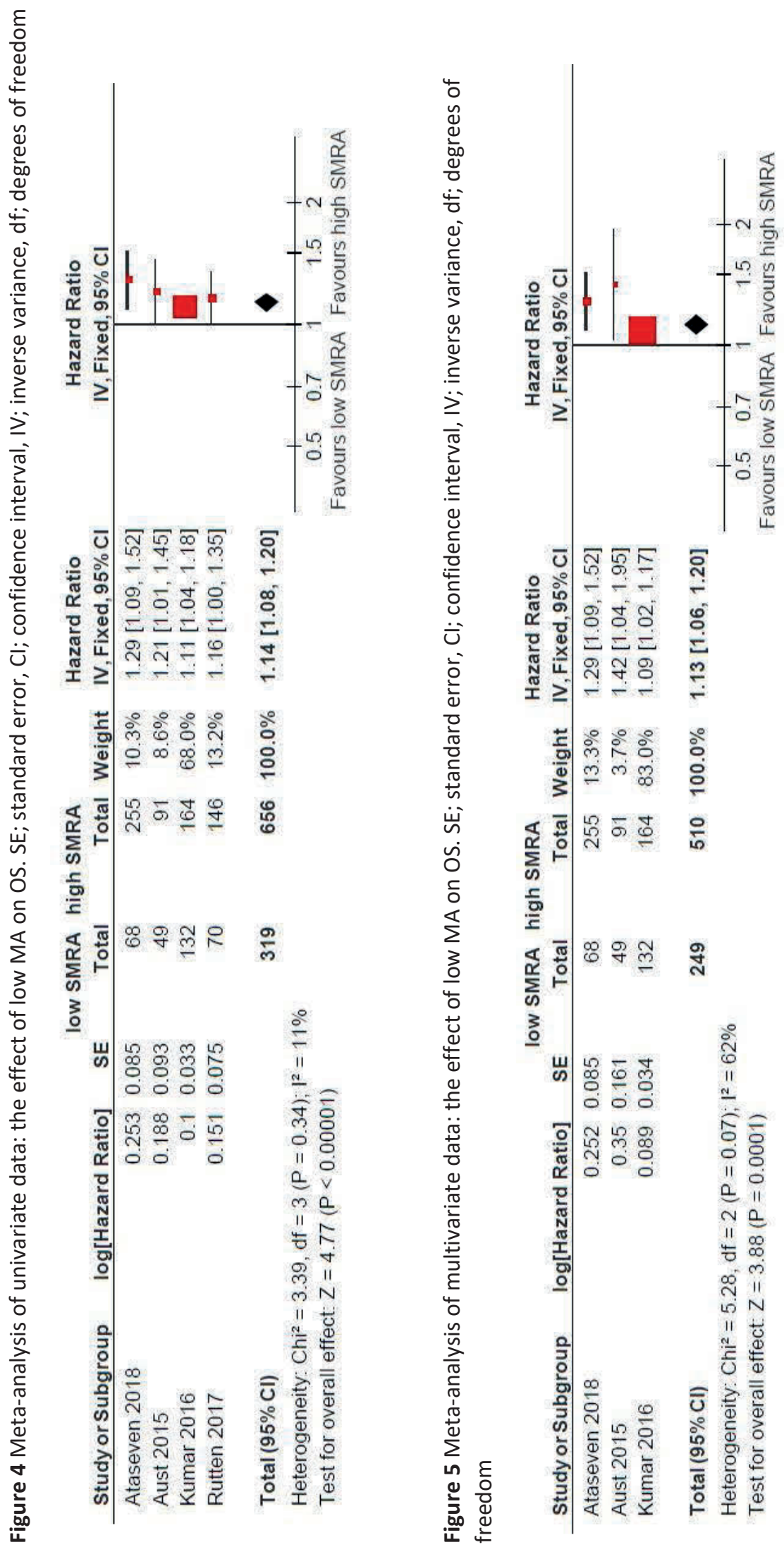




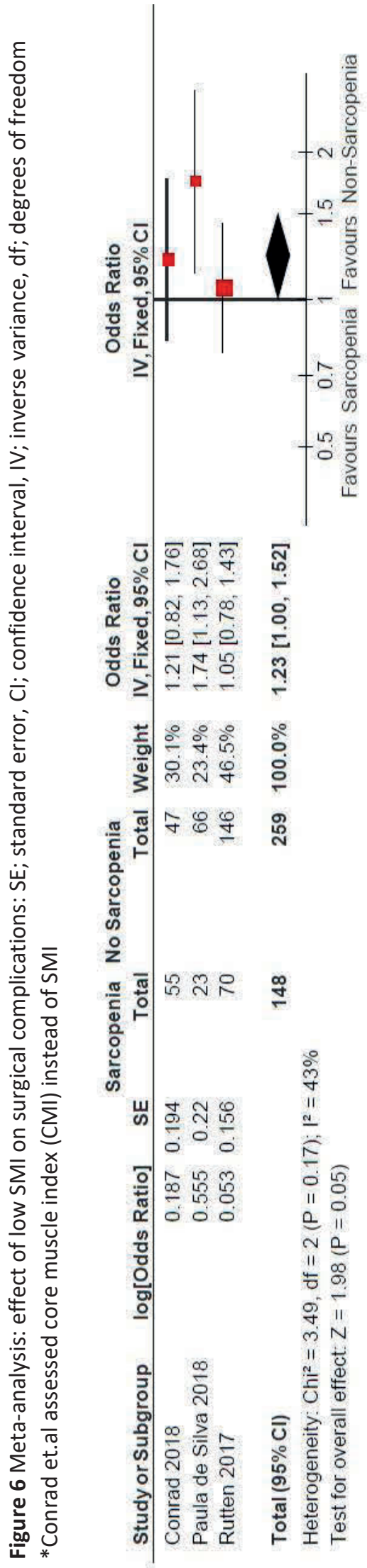




\section{Discussion}

This meta-analysis shows that SMI and SMRA are associated with OS in ovarian cancer patients in both univariate and multivariate analysis. The magnitude of the observed effect in our meta-analysis of multivariate data is likely to be lower than that reported in other studies, because those studies that did not find a significant association between SMI[16, 20, 22] and/or SMRA[17] and survival in univariate analysis did not report multivariate outcomes. The association between SMI and surgical complications was borderline significant in metaanalysis of the univariate data, and meta-analysis of the multivariate data could not be performed because it was only reported in one study[23].

Sarcopenia prevalence estimated by SMI in the included studies of our meta-analysis of ovarian cancer cases has a wide range from 11-54\%; one important reason for which may be related to the use of different cut-offs for assessing sarcopenia $\left(38.5 \mathrm{~cm}^{2} / \mathrm{m}^{2}-41.5 \mathrm{~cm}^{2} / \mathrm{m}^{2}\right)$. Sex-specific cut-offs for defining sarcopenia have previously been made using optimum stratification methods.[15, 33] Optimum stratification methods are based on log-rank statistics. They solve the threshold value of a continuous covariate (SMI) that best separates patients with and without sarcopenia with respect to time to an event outcome.[34] Although cut-offs for females of $38.5 \mathrm{~cm}^{2} / \mathrm{m}^{2}$ [33] for respiratory tract and GI cancer, and $41 \mathrm{~cm}^{2} / \mathrm{m}^{2}$ [15] for lung and GI cancer, are the most widely used, cut-offs used in ovarian cancer studies vary. A meta-analysis of 38 studies of different malignancies (no ovarian cancer patients) by Shachar et al.[8] revealed a range in sarcopenia prevalence of $11-69 \%$. Since the cut-off used to define sarcopenia will directly influence the outcome of associations made between SMI and OS or complication rate, it is vital that a consensus is arrived at. Frequently, cohorts are divided based upon tertiles, quartiles, or standard deviations to discriminate between sarcopenic and non-sarcopenic patients. This seems an acceptable method for comparing patients suffering from more severe sarcopenia with non-sarcopenic patients. In the current cohort, we observed that studies with lower cut-offs, between $38.5-38.73 \mathrm{~cm}^{2} / \mathrm{m}^{2}$, were more likely to report a prognostic effect of sarcopenia.[17, 19] In future assessments of sarcopenia and ovarian cancer survival we propose the use of tertiles, quartiles, or a relatively low cut-off $\left(<38.5 \mathrm{~cm}^{2} / \mathrm{m}^{2}\right)$ to distinguish sarcopenic from non-sarcopenic patients. These measurements should preferably be combined with muscle function assessment according to the recommendations of several international working groups on sarcopenia.[10, 11, 14, 35] 


\section{Supplementary material 4}

Axial CT-scan at the third lumbar level. Skeletal muscle is defined in red. The surface area of the skeletal muscle yields a value in squared centimetres $\left(\mathrm{cm}^{2}\right)$. The skeletal muscle index (SMI in $\mathrm{cm}^{2} / \mathrm{m}^{2}$ ) is derived from skeletal muscle area by dividing it with the squared height of the subject $\left(\mathrm{m}^{2}\right)$. Skeletal muscle radiation attenuation (SMRA) is defined by the mean Hounsfield value of the total skeletal muscle area in red. Picture is taken from Rutten et.al. JCSM, 2017.

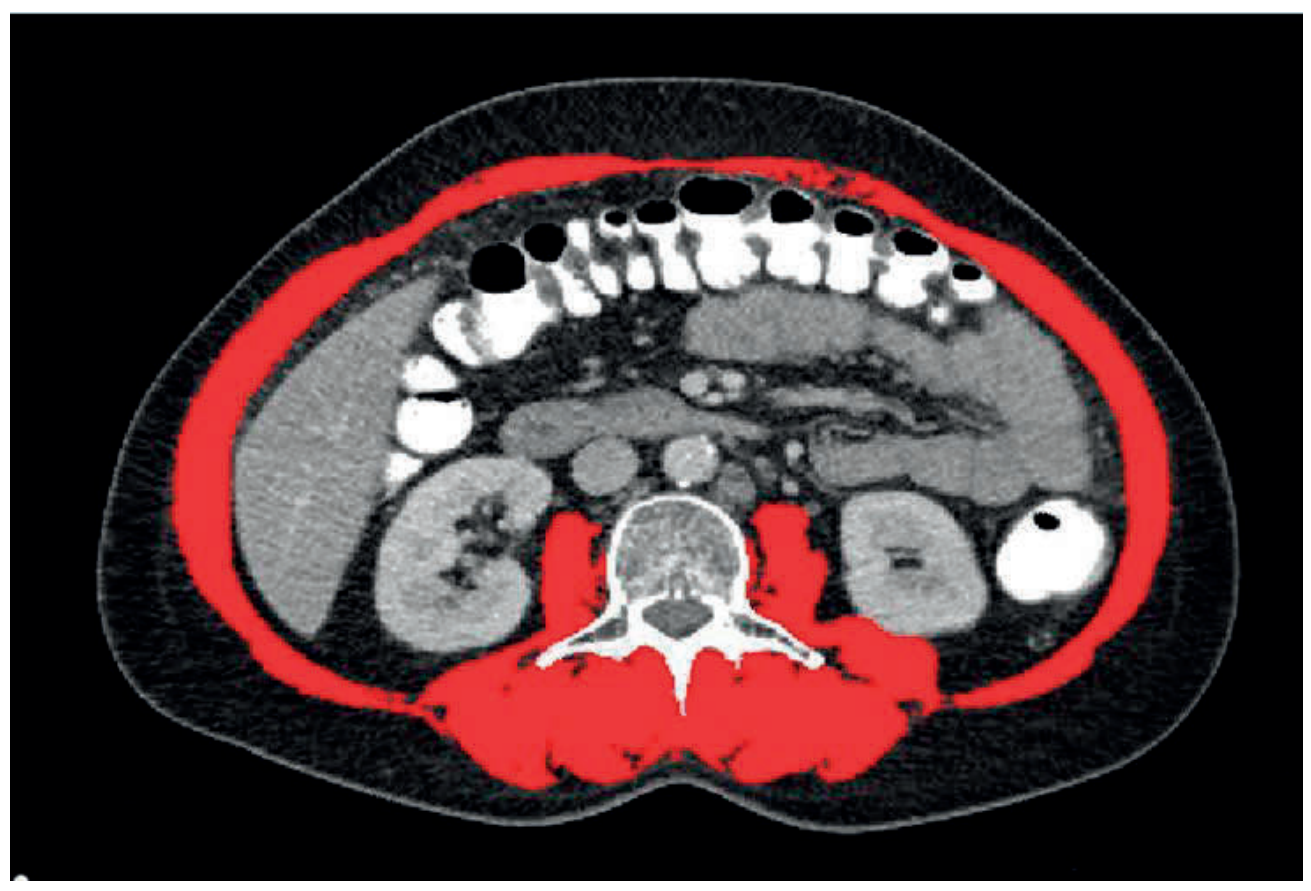

Longitudinal changes in SMI are also used to assess the development of sarcopenia.[16, 19] However, surgery and subsequent recovery considerably affect skeletal muscle mass. One week of bed rest has been shown to lead to a substantial decrease in muscle mass.[36] Nevertheless, since sarcopenia is a dynamic process, the prognostic value of changes in skeletal muscle mass seems greater than assessment of baseline measurements only. One study of end-stage pancreatic cancer patients undergoing chemotherapy did find an association between decreasing skeletal muscle mass and survival.[6] In another cohort of pancreatic cancer patients receiving chemotherapy, in which the prevalence of sarcopenia was very high and the median OS very short, longitudinal decrease of fat and muscle mass were associated with worse OS.[37] In a very large cohort study of stage I-III colorectal cancer patients, a decline in muscle mass was also associated with shorter survival.[38] So far, only two studies have examined longitudinal changes in SMI in ovarian cancer: one study of endstage patients undergoing surgery found no correlation between decreasing skeletal muscle mass and survival [19], while the other examining patients receiving neo-adjuvant chemotherapy concluded that longitudinal decrease in the SMI was predictive for survival.[16] 
The present meta-analysis is the first to report a quantitative assessment of SMI and SMRA and their clinical outcomes in ovarian cancer. The strength of this meta-analysis is that only ovarian cancer patients whose SMI or SMRA were assessed with a lumbar CT-scan were included, creating a homogeneous cohort. A weakness is the fact that included studies used different cut-off points to define sarcopenia, leading to heterogeneity in the reported sarcopenia prevalence. The CMI or psoas muscle index was used for the assessment of skeletal muscle in one included study;[24] however, as the psoas muscle can be affected by spinal pathology, it may be unsuitable for assessing skeletal muscle. Moreover, the psoas represents only a small area of total lumbar skeletal muscle and is difficult to measure accurately, making it an unreliable substitute for the total lumbar skeletal muscle area.[39, 40] Although the contrast phase used for the CT-scan does not majorly influence SMI values[41, 42], it does greatly affect SMRA. Since the contrast phase is not mentioned by any of the studies assessing SMRA, this is a potential shortcoming. Overall, quality of the evidence as assessed with the GRADE checklist was very low. Additionally, all studies in this meta-analysis were of a retrospective nature. Since all studies were methodologically homogeneous, a fixed effects model was chosen for the meta-analysis. However, use of a random effects model would not have changed the outcome for OS. As assumptions of homogeneity were not violated, subgroup analyses were not performed. All the individual studies were found to have a 'high risk of bias' according to evaluation with the QUIPS tool. We did not assess publication bias using a funnel plot as there were fewer than ten included studies.[43] Publication bias arises when small studies with negative results remain unpublished and although publication bias was not assessed, it may be substantial. Based on this meta-analysis, SMI and SMRA measured by axial CT-scanning at the third or fourth lumbar level currently have little utility as reliable prognostic factors in ovarian cancer.

\section{Conclusion}

Although SMI and SMRA appear to be associated with shorter survival in ovarian cancer patients, the lack of standardized cut-offs for assessing its prevalence hampers the interpretation of this association and its strength. A consensus on standardized cut-off values to define sarcopenia in patients with ovarian cancer needs to be found. Since physical exercise interventions are regarded as effective in preventing sarcopenia and improving physical function in cancer patients, future studies should incorporate information on muscle strength and nutritional assessments. 


\section{Supplementary material}

\section{Supplementary material 1 Full electronic search}

Full electronic search performed in multiple international databases.

- Medline (OvidSP)

- Embase (OvidSP)

- CINAHL (Cumulative Index to Nursing and Allied Health Literature) (EBSCO)

- PEDro

\section{Medline (OvidSP)}

Searched 2019/02/14

\begin{tabular}{|c|c|c|}
\hline & Searches & Results \\
\hline 1 & $\begin{array}{l}\text { cache*.mp. [mp=title, abstract, original title, name of substance word, subject heading word, } \\
\text { floating sub-heading word, keyword heading word, organism supplementary concept word, } \\
\text { protocol supplementary concept word, rare disease supplementary concept word, unique } \\
\text { identifier, synonyms] }\end{array}$ & 9783 \\
\hline 2 & $\begin{array}{l}\text { sarcopen*.mp. [mp=title, abstract, original title, name of substance word, subject heading } \\
\text { word, floating sub-heading word, keyword heading word, organism supplementary concept } \\
\text { word, protocol supplementary concept word, rare disease supplementary concept word, unique } \\
\text { identifier, synonyms] }\end{array}$ & 5026 \\
\hline 3 & $\begin{array}{l}\text { muscle*.mp. [mp=title, abstract, original title, name of substance word, subject heading word, } \\
\text { floating sub-heading word, keyword heading word, organism supplementary concept word, } \\
\text { protocol supplementary concept word, rare disease supplementary concept word, unique } \\
\text { identifier, synonyms] }\end{array}$ & 817634 \\
\hline 4 & $\begin{array}{l}\text { body composition.mp. [mp=title, abstract, original title, name of substance word, subject } \\
\text { heading word, floating sub-heading word, keyword heading word, organism supplementary } \\
\text { concept word, protocol supplementary concept word, rare disease supplementary concept } \\
\text { word, unique identifier, synonyms] }\end{array}$ & 48635 \\
\hline 5 & $\begin{array}{l}\text { tumo?r.mp. [mp=title, abstract, original title, name of substance word, subject heading word, } \\
\text { floating sub-heading word, keyword heading word, organism supplementary concept word, } \\
\text { protocol supplementary concept word, rare disease supplementary concept word, unique } \\
\text { identifier, synonyms] }\end{array}$ & 1528822 \\
\hline 6 & $\begin{array}{l}\text { malignan*.mp. [mp=title, abstract, original title, name of substance word, subject heading } \\
\text { word, floating sub-heading word, keyword heading word, organism supplementary concept } \\
\text { word, protocol supplementary concept word, rare disease supplementary concept word, unique } \\
\text { identifier, synonyms] }\end{array}$ & 472983 \\
\hline 7 & $\begin{array}{l}\text { neoplas*.mp. [mp=title, abstract, original title, name of substance word, subject heading word, } \\
\text { floating sub-heading word, keyword heading word, organism supplementary concept word, } \\
\text { protocol supplementary concept word, rare disease supplementary concept word, unique } \\
\text { identifier, synonyms] }\end{array}$ & 2687239 \\
\hline
\end{tabular}




\begin{tabular}{|c|c|c|}
\hline 8 & $\begin{array}{l}\text { carci*.mp. [mp=title, abstract, original title, name of substance word, subject heading word, } \\
\text { floating sub-heading word, keyword heading word, organism supplementary concept word, } \\
\text { protocol supplementary concept word, rare disease supplementary concept word, unique } \\
\text { identifier, synonyms] }\end{array}$ & 892419 \\
\hline 9 & $\begin{array}{l}\text { survival.mp. [mp=title, abstract, original title, name of substance word, subject heading word, } \\
\text { floating sub-heading word, keyword heading word, organism supplementary concept word, } \\
\text { protocol supplementary concept word, rare disease supplementary concept word, unique } \\
\text { identifier, synonyms] }\end{array}$ & 1014660 \\
\hline 10 & $\begin{array}{l}\text { prognos*.mp. [mp=title, abstract, original title, name of substance word, subject heading word, } \\
\text { floating sub-heading word, keyword heading word, organism supplementary concept word, } \\
\text { protocol supplementary concept word, rare disease supplementary concept word, unique } \\
\text { identifier, synonyms] }\end{array}$ & 707758 \\
\hline 11 & $\begin{array}{l}\text { outcome.mp. [mp=title, abstract, original title, name of substance word, subject heading word, } \\
\text { floating sub-heading word, keyword heading word, organism supplementary concept word, } \\
\text { protocol supplementary concept word, rare disease supplementary concept word, unique } \\
\text { identifier, synonyms] }\end{array}$ & 1599871 \\
\hline 12 & $\begin{array}{l}\text { prognostic factors.mp. [mp=title, abstract, original title, name of substance word, subject } \\
\text { heading word, floating sub-heading word, keyword heading word, organism supplementary } \\
\text { concept word, protocol supplementary concept word, rare disease supplementary concept } \\
\text { word, unique identifier, synonyms] }\end{array}$ & 51671 \\
\hline 13 & $\begin{array}{l}\text { complicat*.mp. [mp=title, abstract, original title, name of substance word, subject heading } \\
\text { word, floating sub-heading word, keyword heading word, organism supplementary concept } \\
\text { word, protocol supplementary concept word, rare disease supplementary concept word, unique } \\
\text { identifier, synonyms] }\end{array}$ & 2863331 \\
\hline 14 & $\begin{array}{l}\text { cox.mp. [mp=title, abstract, original title, name of substance word, subject heading word, } \\
\text { floating sub-heading word, keyword heading word, organism supplementary concept word, } \\
\text { protocol supplementary concept word, rare disease supplementary concept word, unique } \\
\text { identifier, synonyms] }\end{array}$ & 120529 \\
\hline 15 & $\begin{array}{l}\text { regression.mp. [mp=title, abstract, original title, name of substance word, subject heading } \\
\text { word, floating sub-heading word, keyword heading word, organism supplementary concept } \\
\text { word, protocol supplementary concept word, rare disease supplementary concept word, unique } \\
\text { identifier, synonyms] }\end{array}$ & 614701 \\
\hline 16 & $\begin{array}{l}\text { prospective.mp. [mp=title, abstract, original title, name of substance word, subject heading } \\
\text { word, floating sub-heading word, keyword heading word, organism supplementary concept } \\
\text { word, protocol supplementary concept word, rare disease supplementary concept word, unique } \\
\text { identifier, synonyms] }\end{array}$ & 661898 \\
\hline 17 & $\begin{array}{l}\text { clinical trial.mp. [mp=title, abstract, original title, name of substance word, subject heading } \\
\text { word, floating sub-heading word, keyword heading word, organism supplementary concept } \\
\text { word, protocol supplementary concept word, rare disease supplementary concept word, unique } \\
\text { identifier, synonyms] }\end{array}$ & 650458 \\
\hline 18 & $\begin{array}{l}\text { observational.mp. [mp=title, abstract, original title, name of substance word, subject heading } \\
\text { word, floating sub-heading word, keyword heading word, organism supplementary concept }\end{array}$ & 147746 \\
\hline
\end{tabular}




\begin{tabular}{|c|c|c|}
\hline & $\begin{array}{l}\text { word, protocol supplementary concept word, rare disease supplementary concept word, unique } \\
\text { identifier, synonyms] }\end{array}$ & \\
\hline 19 & $\begin{array}{l}\text { retrospective.mp. [mp=title, abstract, original title, name of substance word, subject heading } \\
\text { word, floating sub-heading word, keyword heading word, organism supplementary concept } \\
\text { word, protocol supplementary concept word, rare disease supplementary concept word, unique } \\
\text { identifier, synonyms] }\end{array}$ & 801183 \\
\hline 20 & exp Cachexia/ & 4854 \\
\hline 21 & exp Body Composition/ & 49943 \\
\hline 22 & exp Ovarian Neoplasms/ or exp Ovary/ & 160985 \\
\hline 23 & exp Neoplasms/ & 3122927 \\
\hline 24 & exp Survival Rate/ or exp Survival/ or exp Progression-Free Survival/ or exp Survival Analysis/ & 401624 \\
\hline 25 & exp Prognosis/ & 1479937 \\
\hline 26 & exp Treatment Outcome/ & 952272 \\
\hline 27 & exp Intraoperative Complications/ or exp Postoperative Complications/ & 539869 \\
\hline 28 & exp Prospective Studies/ & 492685 \\
\hline 29 & exp Clinical Trial/ & 814956 \\
\hline 30 & exp Observational Study/ & 57816 \\
\hline 31 & exp Retrospective Studies/ & 729577 \\
\hline 32 & $\begin{array}{l}\text { ovar*.mp. [mp=title, abstract, original title, name of substance word, subject heading word, } \\
\text { floating sub-heading word, keyword heading word, organism supplementary concept word, } \\
\text { protocol supplementary concept word, rare disease supplementary concept word, unique } \\
\text { identifier, synonyms] }\end{array}$ & 268015 \\
\hline 33 & exp Muscle, Skeletal/ or exp Sarcopenia/ or exp Muscle Strength/ & 270751 \\
\hline 34 & 1 or 2 or 3 or 4 or 20 or 21 or 33 & 905591 \\
\hline 35 & 5 or 6 or 7 or 8 or 23 & 3685391 \\
\hline 36 & 22 or 32 & 279296 \\
\hline 37 & $\begin{array}{l}9 \text { or } 10 \text { or } 11 \text { or } 12 \text { or } 13 \text { or } 14 \text { or } 15 \text { or } 16 \text { or } 17 \text { or } 18 \text { or } 19 \text { or } 24 \text { or } 25 \text { or } 26 \text { or } 27 \text { or } 28 \text { or } 29 \text { or } \\
30 \text { or } 31\end{array}$ & 6468076 \\
\hline 38 & 34 and 35 and 36 and 37 & 1121 \\
\hline
\end{tabular}




\section{Embase (OvidSP)}

\section{Searched 2019/02/14}

\begin{tabular}{|c|c|c|}
\hline & Searches & Results \\
\hline 1 & $\begin{array}{l}\text { cache*.mp. [mp=title, abstract, heading word, drug trade name, original title, device } \\
\text { manufacturer, drug manufacturer, device trade name, keyword, floating subheading word, } \\
\text { candidate term word] }\end{array}$ & 18664 \\
\hline 2 & $\begin{array}{l}\text { sarcop*.mp. [mp=title, abstract, heading word, drug trade name, original title, device } \\
\text { manufacturer, drug manufacturer, device trade name, keyword, floating subheading word, } \\
\text { candidate term word] }\end{array}$ & 43891 \\
\hline 3 & $\begin{array}{l}\text { muscle*.mp. [mp=title, abstract, heading word, drug trade name, original title, device } \\
\text { manufacturer, drug manufacturer, device trade name, keyword, floating subheading word, } \\
\text { candidate term word] }\end{array}$ & 1446460 \\
\hline 4 & $\begin{array}{l}\text { body composition.mp. [mp=title, abstract, heading word, drug trade name, original title, device } \\
\text { manufacturer, drug manufacturer, device trade name, keyword, floating subheading word, } \\
\text { candidate term word] }\end{array}$ & 62604 \\
\hline 5 & $\begin{array}{l}\text { ovar*.mp. [mp=title, abstract, heading word, drug trade name, original title, device } \\
\text { manufacturer, drug manufacturer, device trade name, keyword, floating subheading word, } \\
\text { candidate term word] }\end{array}$ & 379168 \\
\hline 6 & $\begin{array}{l}\text { cancer.mp. [mp=title, abstract, heading word, drug trade name, original title, device } \\
\text { manufacturer, drug manufacturer, device trade name, keyword, floating subheading word, } \\
\text { candidate term word] }\end{array}$ & 3161838 \\
\hline 7 & $\begin{array}{l}\text { malignan*.mp. [mp=title, abstract, heading word, drug trade name, original title, device } \\
\text { manufacturer, drug manufacturer, device trade name, keyword, floating subheading word, } \\
\text { candidate term word] }\end{array}$ & 798846 \\
\hline 8 & $\begin{array}{l}\text { neoplas*.mp. [mp=title, abstract, heading word, drug trade name, original title, device } \\
\text { manufacturer, drug manufacturer, device trade name, keyword, floating subheading word, } \\
\text { candidate term word] }\end{array}$ & 892034 \\
\hline 9 & $\begin{array}{l}\text { tumo?r.mp. [mp=title, abstract, heading word, drug trade name, original title, device } \\
\text { manufacturer, drug manufacturer, device trade name, keyword, floating subheading word, } \\
\text { candidate term word] }\end{array}$ & 2613898 \\
\hline 10 & $\begin{array}{l}\text { carci*.mp. [mp=title, abstract, heading word, drug trade name, original title, device } \\
\text { manufacturer, drug manufacturer, device trade name, keyword, floating subheading word, } \\
\text { candidate term word] }\end{array}$ & 1382976 \\
\hline 11 & $\begin{array}{l}\text { survival.mp. [mp=title, abstract, heading word, drug trade name, original title, device } \\
\text { manufacturer, drug manufacturer, device trade name, keyword, floating subheading word, } \\
\text { candidate term word] }\end{array}$ & 1523590 \\
\hline
\end{tabular}




\begin{tabular}{|c|c|c|}
\hline 12 & $\begin{array}{l}\text { prognos*.mp. [mp=title, abstract, heading word, drug trade name, original title, device } \\
\text { manufacturer, drug manufacturer, device trade name, keyword, floating subheading word, } \\
\text { candidate term word] }\end{array}$ & 1008266 \\
\hline 13 & $\begin{array}{l}\text { outcome.mp. [mp=title, abstract, heading word, drug trade name, original title, device } \\
\text { manufacturer, drug manufacturer, device trade name, keyword, floating subheading word, } \\
\text { candidate term word] }\end{array}$ & 2284124 \\
\hline 14 & $\begin{array}{l}\text { complicat*.mp. [mp=title, abstract, heading word, drug trade name, original title, device } \\
\text { manufacturer, drug manufacturer, device trade name, keyword, floating subheading word, } \\
\text { candidate term word] }\end{array}$ & 2827748 \\
\hline 15 & $\begin{array}{l}\text { cox.mp. [mp=title, abstract, heading word, drug trade name, original title, device manufacturer, } \\
\text { drug manufacturer, device trade name, keyword, floating subheading word, candidate term } \\
\text { word] }\end{array}$ & 228213 \\
\hline 16 & $\begin{array}{l}\text { regression.mp. [mp=title, abstract, heading word, drug trade name, original title, device } \\
\text { manufacturer, drug manufacturer, device trade name, keyword, floating subheading word, } \\
\text { candidate term word] }\end{array}$ & 1005686 \\
\hline 17 & $\begin{array}{l}\text { prospective.mp. [mp=title, abstract, heading word, drug trade name, original title, device } \\
\text { manufacturer, drug manufacturer, device trade name, keyword, floating subheading word, } \\
\text { candidate term word] }\end{array}$ & 924058 \\
\hline 18 & $\begin{array}{l}\text { clinical trial.mp. [mp=title, abstract, heading word, drug trade name, original title, device } \\
\text { manufacturer, drug manufacturer, device trade name, keyword, floating subheading word, } \\
\text { candidate term word] }\end{array}$ & 1458606 \\
\hline 19 & $\begin{array}{l}\text { observational. } \mathrm{mp} \text {. [mp=title, abstract, heading word, drug trade name, original title, device } \\
\text { manufacturer, drug manufacturer, device trade name, keyword, floating subheading word, } \\
\text { candidate term word] }\end{array}$ & 274780 \\
\hline 20 & $\begin{array}{l}\text { retrospective.mp. [mp=title, abstract, heading word, drug trade name, original title, device } \\
\text { manufacturer, drug manufacturer, device trade name, keyword, floating subheading word, } \\
\text { candidate term word] }\end{array}$ & 1046679 \\
\hline 21 & exp cachexia/ or exp malnutrition/ & 144031 \\
\hline 22 & $\begin{array}{l}\text { exp skeletal muscle/ or exp muscle atrophy/ or exp muscle strength/ or exp sarcopenia/ or exp } \\
\text { muscle mass/ or exp body composition/ }\end{array}$ & 450254 \\
\hline 23 & exp malignant neoplasm/ or exp neoplasm/ & 4043244 \\
\hline 24 & $\begin{array}{l}\text { exp short term survival/ or exp survival/ or exp survival rate/ or exp survival time/ or exp event } \\
\text { free survival/ or exp long term survival/ or exp overall survival/ or exp disease free survival/ or } \\
\text { exp cancer survival/ or exp median survival time/ or exp survival prediction/ or exp recurrence } \\
\text { free survival/ or exp progression free survival/ }\end{array}$ & 991486 \\
\hline 25 & $\begin{array}{l}\text { exp ovary metastasis/ or exp ovary cancer/ or exp ovary tumor/ or exp ovary/ or exp ovary cyst/ } \\
\text { or exp ovary adenocarcinoma/ or exp ovary disease/ or exp ovary carcinoma/ }\end{array}$ & 321032 \\
\hline
\end{tabular}




\begin{tabular}{|c|c|c|}
\hline 26 & $\begin{array}{l}\text { exp muscle fatigue/ or exp muscle/ or exp muscle function/ or exp muscle atrophy/ or exp } \\
\text { muscle mass/ or exp skeletal muscle/ }\end{array}$ & 717897 \\
\hline 27 & exp prognosis/ or exp cancer prognosis/ & 644990 \\
\hline 28 & exp adverse outcome/ or exp clinical outcome/ or exp treatment outcome/ & 1521086 \\
\hline 29 & $\begin{array}{l}\text { exp infection complication/ or exp postoperative complication/ or exp peroperative } \\
\text { complication/ or exp wound complication/ or exp complication/ or exp infectious complication/ } \\
\text { or exp preoperative complication/ }\end{array}$ & 983888 \\
\hline 30 & $\begin{array}{l}\text { exp multiple regression/ or exp nonlinear regression analysis/ or exp logistic regression } \\
\text { analysis/ or exp linear regression analysis/ or exp multivariate logistic regression analysis/ or } \\
\text { exp regression analysis/ or exp multiple linear regression analysis/ }\end{array}$ & 435327 \\
\hline 31 & exp prospective study/ & 500390 \\
\hline 32 & exp retrospective study/ & 737382 \\
\hline 33 & clinical trial/ & 954308 \\
\hline 34 & exp observational study/ or exp observational method/ & 166943 \\
\hline 35 & 1 or 2 or 3 or 4 or 21 or 22 or 26 & 1766241 \\
\hline 36 & 5 or 25 & 445784 \\
\hline 37 & 6 or 7 or 8 or 9 or 10 or 23 & 5301304 \\
\hline 38 & $\begin{array}{l}11 \text { or } 12 \text { or } 13 \text { or } 14 \text { or } 15 \text { or } 16 \text { or } 17 \text { or } 18 \text { or } 19 \text { or } 20 \text { or } 24 \text { or } 27 \text { or } 28 \text { or } 29 \text { or } 30 \text { or } 31 \text { or } 32 \\
\text { or } 33 \text { or } 34\end{array}$ & 8466743 \\
\hline 39 & 35 and 36 and 37 and 38 & 3044 \\
\hline
\end{tabular}

\section{CINAHL (Cumulative Index to Nursing and Allied Health Literature) (EBSCO)}

\section{Searched 2019/02/14}

\begin{tabular}{|l|l||l|l|}
\hline $\begin{array}{l}\text { Search } \\
\text { ID\# }\end{array}$ & Search Terms & Search Options & Results \\
\hline S7 & S1 AND S2 AND S3 AND S6 & Search modes - & (74) \\
\hline S6 & S4 OR S5 & Search modes - & $(1,829,067)$ \\
\hline
\end{tabular}




\begin{tabular}{|l|l||l|l|}
\hline S5 & $\begin{array}{l}\text { MH survival rate OR MH survival OR MH progression-free survival OR } \\
\text { MH survival analysis OR MH prognosis OR MH treatment outcome OR } \\
\text { MH intraoperative complications OR MH postoperative complications } \\
\text { OR MH prospective studies OR MH clinical trial OR MH observational } \\
\text { study OR MH retrospective studies }\end{array}$ & $\begin{array}{l}\text { Search modes - } \\
\text { Boolean/Phrase }\end{array}$ & $(503,613)$ \\
\hline S4 & $\begin{array}{l}\text { survival OR prognos* OR outcome OR prognostic factors OR } \\
\text { complicat* OR cox OR regression OR prospective OR clinical trial OR } \\
\text { observational OR retrospective }\end{array}$ & $\begin{array}{l}\text { Search modes - } \\
\text { Boolean/Phrase }\end{array}$ & $(1,829,067)$ \\
\hline S3 & $\begin{array}{l}\text { cancer OR tumo\#r OR malignan* OR neoplas* OR carci* OR MH } \\
\text { neoplasms }\end{array}$ & Search modes - \\
Boolean/Phrase & $(567,635)$ \\
\hline S2 & $\begin{array}{l}\text { ovar* OR MH ovarian neoplasms OR MH ovary } \\
\text { S1 }\end{array}$ & $\begin{array}{l}\text { saropen* OR MH skeletal muscle OR MH muscle strength OR MH } \\
\text { suscle* OR body composition }\end{array}$ & Search modes - \\
\hline Boolean/Phrase & $(28,029)$ \\
\hline
\end{tabular}

\section{PEDro (Physiotherapy Evidence Database)}

\section{Searched 2019/02/15}

Subdiscipline: oncology

Terms in abstract and title: cachex* 5

Terms in abstract and title: sarco* 6

Terms in abstract and title: ovar* 12 


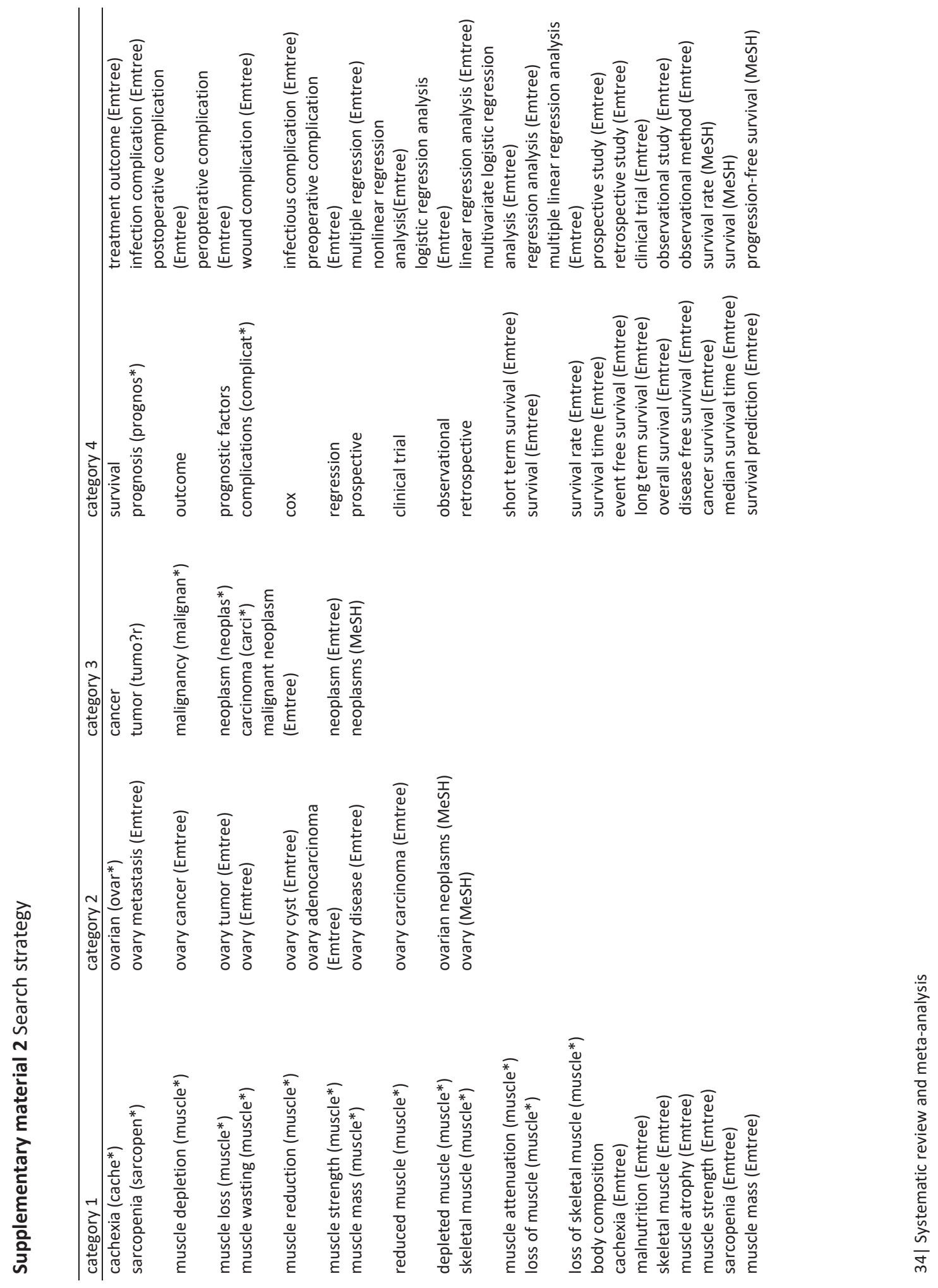



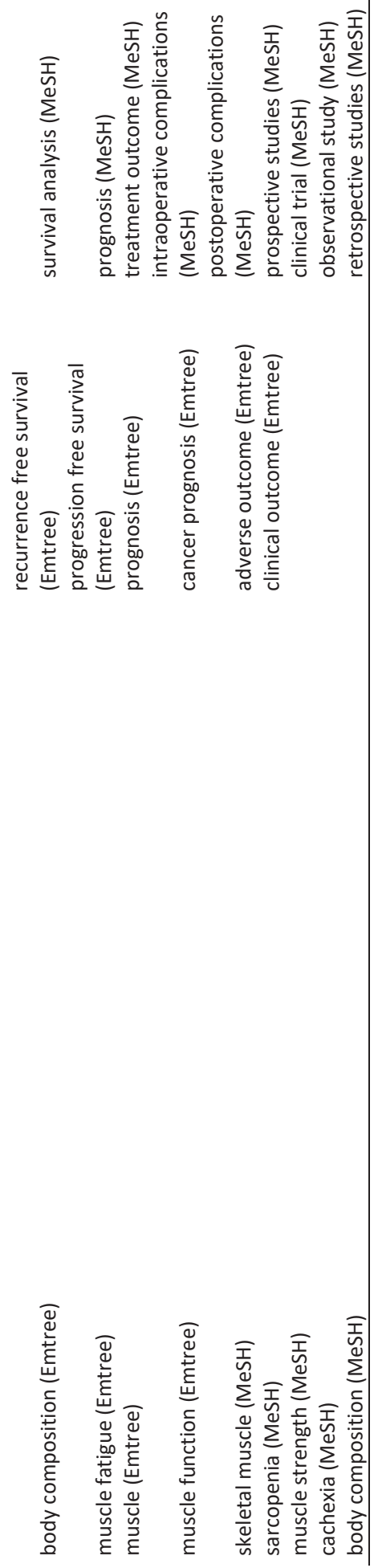


\begin{tabular}{|c|c|c|c|c|c|c|c|c|c|c|c|c|c|c|c|c|c|}
\hline & 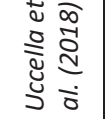 & & 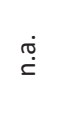 & $>$ & $>$ & $>$ & $>$ & $>$ & $\left|\begin{array}{c}n \\
\frac{0}{0} \\
3 \\
0 \\
-3\end{array}\right|$ & $x$ & $\times$ & $>$ & $x$ & $\times$ & $\left|\begin{array}{l}\frac{6}{0} \\
\frac{.0}{0} \\
\frac{0}{60} \\
\underline{\underline{x}} \\
\end{array}\right|$ & $>$ & $>$ \\
\hline & 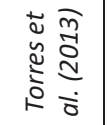 & & $\stackrel{\text { ஷேं }}{\check{\Sigma}}$ & $>$ & $>$ & $>$ & $>$ & $>$ & $\left|\begin{array}{c}n \\
\frac{0}{0} \\
3 \\
0 \\
-1\end{array}\right|$ & $x$ & $\times$ & $>$ & $\times$ & $\times$ & $\left|\begin{array}{|c|}\tilde{n} \\
\frac{0}{0} \\
\frac{0}{0.00} \\
\frac{.00}{I}\end{array}\right|$ & $>$ & $>$ \\
\hline & 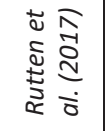 & & ֻோ & $>$ & $>$ & $>$ & $>$ & $>$ & $\mid \begin{array}{c}0 \\
\frac{0}{0} \\
3 \\
0 \\
- \\
\end{array}$ & $x$ & $\times$ & $>$ & $\times$ & $\times$ & $\mid$\begin{tabular}{l|}
$\frac{n}{0}$ \\
$\frac{.0}{0}$ \\
$\frac{0}{600}$ \\
$\underline{\underline{I 0}}$ \\
\end{tabular} & $>$ & $>$ \\
\hline & 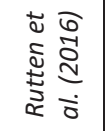 & & 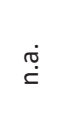 & $>$ & $>$ & $>$ & $>$ & $>$ & $\mid \begin{array}{c}0 \\
\frac{0}{0} \\
3 \\
0 \\
-3\end{array}$ & $x$ & $\times$ & $>$ & $x$ & $\times$ & 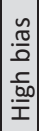 & $>$ & $>$ \\
\hline & 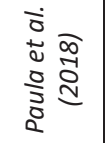 & & 官 & $>$ & $>$ & $>$ & $>$ & $>$ & $\left|\begin{array}{c}0 \\
\frac{0}{0} \\
3 \\
3 \\
-3\end{array}\right|$ & $x$ & $\times$ & $>$ & $\times$ & $\times$ & $\left|\begin{array}{|c|}\frac{n}{0} \\
\frac{.0}{0} \\
\frac{7}{000} \\
\frac{00}{1}\end{array}\right|$ & $>$ & $>$ \\
\hline & 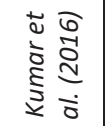 & & 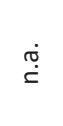 & $>$ & $>$ & $>$ & $>$ & $>$ & $\mid \begin{array}{c}n \\
\frac{0}{0} \\
3 \\
0 \\
-3\end{array}$ & $x$ & $\times$ & $>$ & $\times$ & $\times$ & 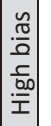 & $>$ & $>$ \\
\hline & 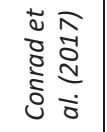 & & ֻோ & $>$ & $>$ & $>$ & $>$ & $>$ & \begin{tabular}{|c|}
0 \\
$\frac{\pi}{0}$ \\
3 \\
0 \\
-3
\end{tabular} & $x$ & $\dot{x}$ & $>$ & $\times$ & $\times$ & 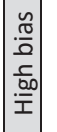 & $>$ & $>$ \\
\hline $\begin{array}{l}\bar{n} \\
\frac{0}{\partial} \\
\frac{0}{\tilde{y}}\end{array}$ & 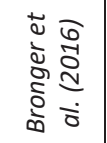 & & ֻோ & $>$ & $>$ & $>$ & $>$ & $>$ & \begin{tabular}{|c|c}
$\frac{n}{0}$ \\
$\frac{0}{0}$ \\
3 \\
3 \\
0
\end{tabular} & $x$ & $\times$ & $>$ & $\times$ & $\times$ & 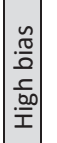 & $>$ & $>$ \\
\hline 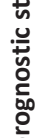 & 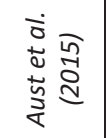 & & 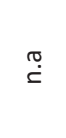 & $>$ & $>$ & $>$ & $>$ & $>$ & $\left|\begin{array}{c}0 \\
\frac{\pi}{0} \\
3 \\
3 \\
0\end{array}\right|$ & $\times$ & $\times$ & $>$ & $\times$ & $\times$ & $\mid \begin{array}{l}\frac{n}{0} \\
\frac{0}{0} \\
\frac{1}{0.0} \\
\frac{.00}{1}\end{array}$ & $>$ & $>$ \\
\hline 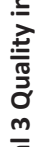 & 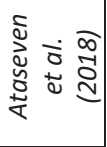 & & $\stackrel{\text { வீं }}{\check{c}}$ & $>$ & $>$ & $>$ & $>$ & $>$ & $\left|\begin{array}{c}0 \\
\frac{\pi}{0} \\
3 \\
3 \\
0\end{array}\right|$ & $\times$ & $\times$ & $>$ & $\times$ & $\times$ & $\mid \begin{array}{l}\frac{n}{0} \\
\frac{.0}{0} \\
\frac{2}{000} \\
\bar{I}\end{array}$ & 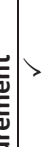 & $>$ \\
\hline 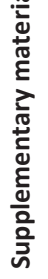 & & 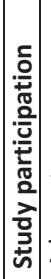 & 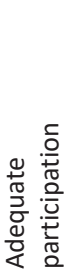 & $\mid \begin{array}{l}c \\
.0 \\
0 \\
0 \\
0 \\
0 \\
0 \\
0 \\
4 \\
0 \\
0 \\
0 \\
0 \\
0 \\
0 \\
0\end{array}$ & 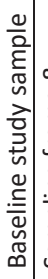 & 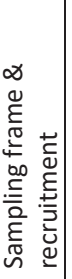 & 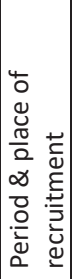 & 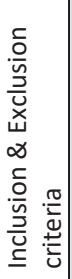 & 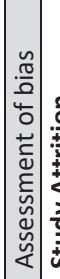 & 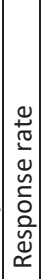 & 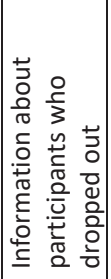 & 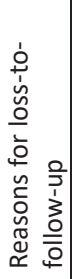 & 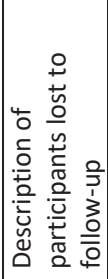 & 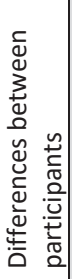 & 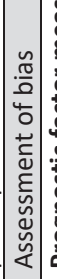 & 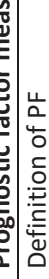 & 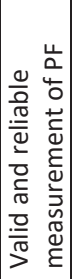 \\
\hline
\end{tabular}

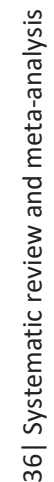




\begin{tabular}{|c|c|c|c|c|c|c|c|c|c|c|c|c|c|}
\hline$>$ & $>$ & - & $\sim$ & $\left|\begin{array}{c}0 \\
\frac{0}{0} \\
3 \\
0 \\
-3\end{array}\right|$ & $>$ & $>$ & $>$ & $\left|\begin{array}{l}0 \\
\frac{0}{0} \\
3 \\
0 \\
0\end{array}\right|$ & $>$ & - & $>$ & $>$ & n. \\
\hline$>$ & $>$ & $>$ & $>$ & $\left|\begin{array}{c}n \\
\frac{0}{0} \\
3 \\
0 \\
-3\end{array}\right|$ & - & $>$ & $>$ & $\left|\begin{array}{l}0 \\
\frac{0}{0} \\
3 \\
0 \\
0\end{array}\right|$ & $>$ & - & $>$ & $>$ & $>$ \\
\hline$>$ & $>$ & $>$ & $>$ & $\left|\begin{array}{c}n \\
\frac{\pi}{0} \\
3 \\
3 \\
0 \\
-1\end{array}\right|$ & $>$ & $>$ & $>$ & $\mid \begin{array}{c}0 \\
\frac{\pi}{0} \\
3 \\
3 \\
0 \\
-3\end{array}$ & $>$ & - & $>$ & $>$ & ^. \\
\hline$>$ & $>$ & $>$ & $>$ & $\left|\begin{array}{c}0 \\
\frac{0}{0} \\
3 \\
0 \\
0\end{array}\right|$ & - & $>$ & $>$ & $\mid \begin{array}{c}0 \\
\frac{0}{0} \\
3 \\
0 \\
0 \\
-1\end{array}$ & - & - & $>$ & $>$ & n. \\
\hline$>$ & $>$ & $>$ & $\sim$ & $\left|\begin{array}{c}0 \\
\frac{0}{0} \\
3 \\
0 \\
0\end{array}\right|$ & $>$ & $>$ & $>$ & $\mid$\begin{tabular}{|c|c}
$\frac{n}{0}$ \\
$\frac{0}{0}$ \\
3 \\
0 \\
0
\end{tabular} & - & - & $>$ & $>$ & ^. \\
\hline$>$ & $>$ & $>$ & $\cdots$ & $\left|\begin{array}{l}0 \\
\frac{0}{0} \\
3 \\
0 \\
-3\end{array}\right|$ & $>$ & $>$ & $>$ & $\left|\begin{array}{l}\frac{n}{0} \\
\frac{0}{0} \\
3 \\
0 \\
-3\end{array}\right|$ & $>$ & - & $>$ & $>$ & ^. \\
\hline$>$ & $>$ & $>$ & $\cdots$ & $\mid \begin{array}{l}0 \\
\frac{\pi}{0} \\
3 \\
0 \\
0\end{array}$ & $>$ & $>$ & $>$ & $\mid$\begin{tabular}{c|}
$\frac{n}{0}$ \\
$\frac{0}{0}$ \\
3 \\
0 \\
-3
\end{tabular} & - & - & $>$ & $>$ & n. \\
\hline$>$ & $>$ & $>$ & $\sim$ & \begin{tabular}{|c|c}
$\frac{0}{0}$ \\
$\frac{0}{0}$ \\
3 \\
0 \\
0
\end{tabular} & - & $>$ & $>$ & $\mid \begin{array}{c}\frac{n}{0} \\
\frac{0}{0} \\
3 \\
0 \\
0\end{array}$ & $>$ & - & $>$ & $>$ & م. \\
\hline$>$ & $>$ & $>$ & - & $\mid \begin{array}{c}\frac{n}{0} \\
\frac{0}{0} \\
3 \\
0 \\
-1\end{array}$ & $>$ & $>$ & $>$ & $\left|\begin{array}{c|}\frac{n}{0} \\
\frac{0}{0} \\
3 \\
0 \\
0\end{array}\right|$ & $>$ & - & $>$ & $>$ & - \\
\hline$>$ & $>$ & $>$ & c. & $\begin{array}{l}0 \\
\frac{0}{0} \\
3 \\
3 \\
-3\end{array}$ & $>$ & $>$ & $>$ & \begin{tabular}{|c|}
$\mid$ \\
$\frac{0}{0}$ \\
3 \\
0 \\
0 \\
\end{tabular} \mid & $>$ & - & $>$ & $>$ & 几. \\
\hline 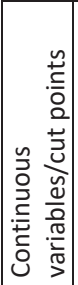 & 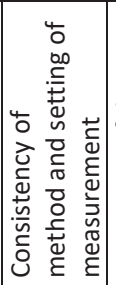 & 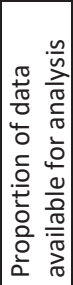 & 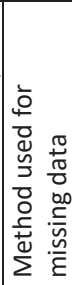 & 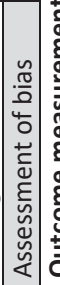 & 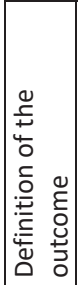 & 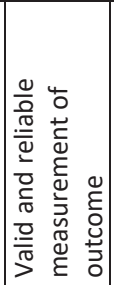 & 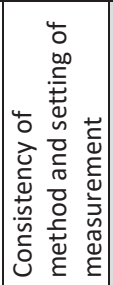 & 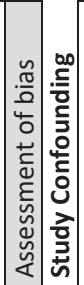 & 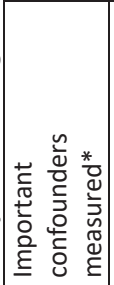 & 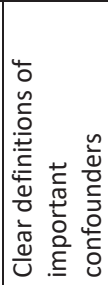 & 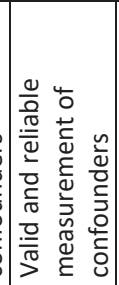 & 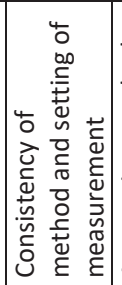 & 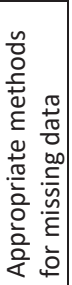 \\
\hline
\end{tabular}

$\frac{n}{n}$
$\frac{N}{d}$
$\frac{1}{0}$
$\frac{0}{0}$
$\frac{1}{U}$ 


$$
\lfloor
$$




\section{References}

1. Timmermans, M., et al., No improvement in long-term survival for epithelial ovarian cancer patients: A population-based study between 1989 and 2014 in the Netherlands. Eur J Cancer, 2018. 88: p. 31-37.

2. Tanyi, J.L., et al., Personalized cancer vaccine effectively mobilizes antitumor $T$ cell immunity in ovarian cancer. Sci Transl Med, 2018. 10(436).

3. Genta, S., et al., Reprogramming T-cells for adoptive immunotherapy of ovarian cancer. Expert Opin Biol Ther, 2018. 18(4): p. 359-367.

4. Cornelison, R., D.C. Llaneza, and C.N. Landen, Emerging Therapeutics to Overcome Chemoresistance in Epithelial Ovarian Cancer: A Mini-Review. Int J Mol Sci, 2017. 18(10).

5. van Driel, W.J., et al., Hyperthermic Intraperitoneal Chemotherapy in Ovarian Cancer. N Engl J Med, 2018. 378(3): p. 230-240.

6. Choi, Y., et al., Skeletal Muscle Depletion Predicts the Prognosis of Patients with Advanced Pancreatic Cancer Undergoing Palliative Chemotherapy, Independent of Body Mass Index. PLoS One, 2015. 10(10): p. e0139749.

7. Levolger, S., et al., Systematic review of sarcopenia in patients operated on for gastrointestinal and hepatopancreatobiliary malignancies. Br J Surg, 2015. 102(12): p. 144858.

8. Shachar, S.S., et al., Prognostic value of sarcopenia in adults with solid tumours: A metaanalysis and systematic review. Eur J Cancer, 2016. 57: p. 58-67.

9. Fukushima, H., et al., Prognostic significance of sarcopenia in upper tract urothelial carcinoma patients treated with radical nephroureterectomy. Cancer Med, 2016. 5(9): p. 2213-20.

10. Cruz-Jentoft, A.J., et al., Sarcopenia: European consensus on definition and diagnosis: Report of the European Working Group on Sarcopenia in Older People. Age Ageing, 2010. 39(4): p. 412-23.

11. Muscaritoli, M., et al., Consensus definition of sarcopenia, cachexia and pre-cachexia: joint document elaborated by Special Interest Groups (SIG) "cachexia-anorexia in chronic wasting diseases" and "nutrition in geriatrics". Clin Nutr, 2010. 29(2): p. 154-9.

12. Fearon, K., et al., Definition and classification of cancer cachexia: an international consensus. Lancet Oncol, 2011. 12(5): p. 489-95.

13. Fearon, K., J. Arends, and V. Baracos, Understanding the mechanisms and treatment options in cancer cachexia. Nat Rev Clin Oncol, 2013. 10(2): p. 90-9.

14. Cesari, M., et al., Biomarkers of sarcopenia in clinical trials-recommendations from the International Working Group on Sarcopenia. J Cachexia Sarcopenia Muscle, 2012. 3(3): p. 181-90.

15. Martin, L., et al., Cancer cachexia in the age of obesity: skeletal muscle depletion is a powerful prognostic factor, independent of body mass index. J Clin Oncol, 2013. 31(12): p. 1539-47.

16. Rutten, I.J., et al., Loss of skeletal muscle during neoadjuvant chemotherapy is related to decreased survival in ovarian cancer patients. J Cachexia Sarcopenia Muscle, 2016. 7(4): p. 458-66.

17. Rutten, I.J., et al., The influence of sarcopenia on survival and surgical complications in ovarian cancer patients undergoing primary debulking surgery. Eur J Surg Oncol, 2017. 43(4): p. 717-724.

18. Aust, S., et al., Skeletal Muscle Depletion and Markers for Cancer Cachexia Are Strong Prognostic Factors in Epithelial Ovarian Cancer. PLoS One, 2015. 10(10): p. e0140403.

19. Bronger, H., et al., Sarcopenia in Advanced Serous Ovarian Cancer. Int J Gynecol Cancer, 2017. 27(2): p. 223-232. 
20. Kumar, A., et al., Muscle composition measured by CT scan is a measurable predictor of overall survival in advanced ovarian cancer. Gynecol Oncol, 2016. 142(2): p. 311-6.

21. Torres, M.L., et al., Nutritional status, CT body composition measures and survival in ovarian cancer. Gynecol Oncol, 2013. 129(3): p. 548-53.

22. Ataseven, B., et al., Skeletal Muscle Attenuation (Sarcopenia) Predicts Reduced Overall Survival in Patients with Advanced Epithelial Ovarian Cancer Undergoing Primary Debulking Surgery. Ann Surg Oncol, 2018. 25(11): p. 3372-3379.

23. Silva de Paula, N., et al., Sarcopenia and Skeletal Muscle Quality as Predictors of Postoperative Complication and Early Mortality in Gynecologic Cancer. Int J Gynecol Cancer, 2018. 28(2): p. 412-420.

24. Conrad, L.B., et al., Pre-operative core muscle index in combination with hypoalbuminemia is associated with poor prognosis in advanced ovarian cancer. J Surg Oncol, 2018. 117(5): p. 1020-1028.

25. Uccella, S., et al., Assessment of preoperative nutritional status using BIA-derived phase angle (PhA) in patients with advanced ovarian cancer: Correlation with the extent of cytoreduction and complications. Gynecol Oncol, 2018. 149(2): p. 263-269.

26. Prado, C.M., et al., The association between body composition and toxicities from the combination of Doxil and trabectedin in patients with advanced relapsed ovarian cancer. Appl Physiol Nutr Metab, 2014. 39(6): p. 693-8.

27. Moher, D., et al., Preferred reporting items for systematic reviews and meta-analyses: the PRISMA Statement. Open Med, 2009. 3(3): p. e123-30.

28. Hayden, J.A., P. Cote, and C. Bombardier, Evaluation of the quality of prognosis studies in systematic reviews. Ann Intern Med, 2006. 144(6): p. 427-37.

29. Hayden, J.A., et al., Assessing bias in studies of prognostic factors. Ann Intern Med, 2013. 158(4): p. 280-6.

30. Hayden JA, Tougas ME, Riley R, lles R, Pincus T. Individual recovery expectations and prognosis of outcomes in non-specific low back pain: prognostic factor exemplar review. Cochrane Database of Systematic Reviews 2014, Issue 9.

31. Iorio, A., et al., Use of GRADE for assessment of evidence about prognosis: rating confidence in estimates of event rates in broad categories of patients. BMJ, 2015. 350: p. h870.

32. Parmar, M.K., V. Torri, and L. Stewart, Extracting summary statistics to perform metaanalyses of the published literature for survival endpoints. Stat Med, 1998. 17(24): p. 281534.

33. Prado, C.M., et al., Prevalence and clinical implications of sarcopenic obesity in patients with solid tumours of the respiratory and gastrointestinal tracts: a population-based study. Lancet Oncol, 2008. 9(7): p. 629-35.

34. B Williams, JN Mandrekar, SJ Mandrekar, SS Cha, AF Furth: Finding optimal cutpoints for continuous covariates with binary and time-to-event outcomes. Mayo Foundation Technical Report Series, number 79, Department of Health Sciences Research, Mayo Clinic, Rochester, MN (2006)

35. Morley, J.E., et al., Sarcopenia with limited mobility: an international consensus. J Am Med Dir Assoc, 2011. 12(6): p. 403-9.

36. Dirks, M.L., et al., One Week of Bed Rest Leads to Substantial Muscle Atrophy and Induces Whole-Body Insulin Resistance in the Absence of Skeletal Muscle Lipid Accumulation. Diabetes, 2016. 65(10): p. 2862-75.

37. Kays, J.K., et al., Three cachexia phenotypes and the impact of fat-only loss on survival in FOLFIRINOX therapy for pancreatic cancer. J Cachexia Sarcopenia Muscle, 2018. 9(4): p. 673684.

38. Brown, J.C., et al., The deterioration of muscle mass and radiodensity is prognostic of poor survival in stage I-III colorectal cancer: a population-based cohort study (C-SCANS). J Cachexia Sarcopenia Muscle, 2018. 9(4): p. 664-672. 
39. Baracos, V.E., Psoas as a sentinel muscle for sarcopenia: a flawed premise. J Cachexia Sarcopenia Muscle, 2017. 8(4): p. 527-528.

40. Rutten, I.J.G., et al., Psoas muscle area is not representative of total skeletal muscle area in the assessment of sarcopenia in ovarian cancer. J Cachexia Sarcopenia Muscle, 2017. 8(4): p. 630-638.

41. van Vugt, J.L.A., et al., Contrast-enhancement influences skeletal muscle density, but not skeletal muscle mass, measurements on computed tomography. Clin Nutr, 2018. 37(5): p. 1707-1714.

42. Rollins, K.E., et al., Body composition measurement using computed tomography: Does the phase of the scan matter? Nutrition, 2017. 41: p. 37-44.

43. Sterne, J.A., et al., Recommendations for examining and interpreting funnel plot asymmetry in meta-analyses of randomised controlled trials. BMJ, 2011. 343: p. d4002. 
3 
Psoas muscle area is not representative of total skeletal muscle area in the assessment of sarcopenia in ovarian cancer

Journal of Cachexia, Sarcopenia and Muscle (2017)

Iris Rutten, Jorne Ubachs, Roy Kruitwagen, Regina Beets-Tan, Steven Olde Damink, Toon Van Gorp. 


\begin{abstract}
Background

Computed tomography measurements of total skeletal muscle area can detect changes and predict overall survival (OS) in patients with advanced ovarian cancer. This study investigates whether assessment of psoas muscle area reflects total muscle area and can be used to assess sarcopenia in ovarian cancer patients.
\end{abstract}

\title{
Methods
}

Ovarian cancer patients $(n=150)$ treated with induction chemotherapy and interval debulking were enrolled retrospectively in this longitudinal study. Muscle was measured crosssectionally with computed tomography in three ways: (1) software quantification of total skeletal muscle area (SMA), (2) software quantification of psoas muscle area (PA), and (3) manual measurement of length and width of the psoas muscle to derive the psoas surface area (PLW). Pearson correlation between the different methods was studied. Patients were divided into two groups based on the extent of change in muscle area and agreement was measured with kappa coefficients. Cox-regression was used to test predictors for OS.

\section{Results}

Correlation between SMA and both psoas muscle area measurements was poor $(r=0.52$ and 0.39 for PA and PLW, respectively). After categorising patients into muscle loss or gain, kappa agreement was also poor for all comparisons (all $\mathrm{k}<0.40$ ). In regression analysis, SMA loss was predictive of poor OS (hazard ratio $1.698(95 \% \mathrm{Cl} 1.038-2.778), \mathrm{P}=0.035)$. No relationship with OS was seen for PA or PLW loss.

\section{Conclusions}

Change in psoas muscle area is not representative of total muscle area change and should not be used to substitute total skeletal muscle to predict survival in patients with ovarian cancer. 


\section{Introduction}

Sixty percent of patients diagnosed with epithelial ovarian cancer have primary metastatic disease with a corresponding 5-year survival of only $28 \%$ [1]. Therapeutic options for this advanced disease (International Federation of Gynecology and Obstetrics, FIGO stage IIB-IV) are either primary debulking surgery followed by adjuvant chemotherapy or induction chemotherapy followed by interval debulking surgery. The outcome of debulking surgery is by far the most important prognostic factor for patients with advanced ovarian cancer and surgery should always be aimed at achieving complete removal of macroscopic tumour $[2 ; 3]$. At the same time, recent investigations have led to the discovery that skeletal muscle area changes detected on computed tomography (CT) may be closely related to ovarian cancer survival as well $[4 ; 5]$. In our own ovarian cancer cohort of patients treated with induction chemotherapy and interval debulking surgery we have shown that patients who were able to gain or maintain muscle area during chemotherapy had a significantly better overall survival (OS) than patients who lost muscle area [4]. What became apparent in this study was that a measurement over time was essential to identify muscle loss or sarcopenia. A cross-sectional single time point measurement could not detect change and was thus unable to predict survival [4]. The importance of sarcopenia has scarcely been studied in ovarian cancer and these results have yet to be confirmed in international prospective trials. However, similar results have been found for other cancer types; stable or increasing muscle mass has been reported to relate to a prolonged survival in non-small cell lung cancer, pancreatic cancer and colorectal cancer while a low muscle mass at baseline showed no prognostic significance [68].

Cross-sectional CT measurement of the total skeletal muscle area (SMA) at the level of the third lumbar vertebra (L3) has proven to give a reliable representation of total body muscle mass and has therefore been adopted worldwide for the detection of sarcopenia in cancer patients $[9 ; 10]$. As an alternative for measuring all skeletal muscle visible at $L 3$, one can opt to evaluate the psoas muscle alone. A scientific rationale for using the psoas is not provided by any authors using this muscle for evaluation of sarcopenia, but we speculate that it might have been selected due to ease of identification or possibly because of its functional role as a hip flexor muscle. In case of a decrease in weight-bearing exercise due to physical unfitness or hospitalisation, the psoas muscle is expected to decrease in volume, which can be used as a potential measure of muscle loss. Although imaging software is still needed, measuring the psoas area (PA) alone is easier and less time consuming. This method has been used to predict surgical complications in different cancer types with contrasting results. PA has shown a correlation with post-operative complications in individual studies on colorectal cancer, colorectal liver metastases, kidney cancer, bladder cancer, cholangiocarcinoma and hepatocellular carcinoma [11-16], while this effect was not seen in other pancreatic cancer, endometrial cancer, biliary cancer or sarcoma studies [17-20]. Interestingly, only few cancer studies were able to show a correlation between PA and survival [21-23]. In a small number of non-cancer studies, decreased psoas muscle area has also been correlated with higher rates of morbidity [24-26] and mortality [25; 27-29] in patients undergoing cardiothoracic, gastrointestinal and spinal surgery. To simplify evaluation of muscle area- an even quicker and easier novel method has been reported. By multiplying the length and width of the psoas muscle (PLW), the psoas area can be directly calculated without the need for specialised software. Jones et al. studied 100 patients with colorectal cancer and reported a good correlation between the standard and new method to measure the psoas muscle as well as a good 
correlation between measurements of the SMA in comparison to the standard PA method [11]. Evaluation of change in muscle mass over time is important for ovarian cancer prognosis; patients who are identified adequately could possibly benefit from nutritional or physical interventions. Standard CT measurements of SMA have been able to detect changes and predict OS in patients with ovarian cancer undergoing induction chemotherapy and interval debulking. This study aims to investigate whether two methods to assess the psoas muscle area, PA and PWL, reflect the total muscle area and give a reliable representation of sarcopenia in ovarian cancer with the same accuracy as SMA assessment.

\section{Methods}

This study has been approved by the local Medical Ethics Committee and has been performed in accordance with the ethical standards laid down in the 1964 Declaration of Helsinki. The requirement to obtain informed consent was waived by a Medical Ethics Committee.

\section{Eligible patients}

All patients with advanced ovarian cancer (FIGO 2013 stage IIB-IV) who were treated with induction chemotherapy and interval debulking in the Maastricht University Medical Centre (Maastricht, the Netherlands) between 2000 and 2015 were enrolled in this retrospective study. Patients were eligible for inclusion if they met the following criteria: 1) at least two routine abdominal CT scans were performed, the first before the start of induction chemotherapy and the second before interval debulking (typically after 3-4 cycles of chemotherapy), 2) the quality of both CT scans was sufficient to perform measurements of muscle area, 3) relevant clinical data could be retrieved from the patients' medical records, and 4) follow-up was at least 6 months after diagnosis. Part of this population was used in prior investigations from which the results have been published previously [4].

Patients were divided in age groups $\leq 60$ years, $61-70$ years and $>70$ years according to their age at diagnosis. Surgical outcome was categorised into complete (no visible evidence of macroscopic residual disease), optimal (macroscopic residual disease $\leq 1 \mathrm{~cm}$ ) or incomplete (macroscopic residual disease $>1 \mathrm{~cm}$ ). OS was defined as the period of time between the initial $\mathrm{CT}$ and a patient's death from any cause as reported in national registries. Patients who were still alive at the time of analysis were censored at a fixed date.

\section{CT analysis}

A single axial image corresponding to the $L 3$ vertebral body was selected for each $C T$. For both SMA and PA, SliceOmatic software (v5.0, Tomovision, Montreal, QC, Canada) was used to quantify skeletal muscles within predefined validated boundaries of -29 to +150 Hounsfield Units. For SMA, the entire skeletal muscle area consisting of the abdominal muscles, psoas muscles and paraspinal muscles was demarcated. For PA only the psoas muscle area (right and left) was selected. Following demarcation, the surface areas were quantified automatically in $\mathrm{cm}^{2}$. For PLW, the length and width of the right and left psoas muscle was measured by hand and multiplied to compute the psoas surface area. Right and left psoas muscle were summated to estimate the total psoas length*width area in $\mathrm{cm}^{2}$. Two trained observers (IR and JU) blinded to patient details and clinical outcomes individually applied the three methods to all CT scans (Figure 1). 
Figure 1 Muscle area measurement methods

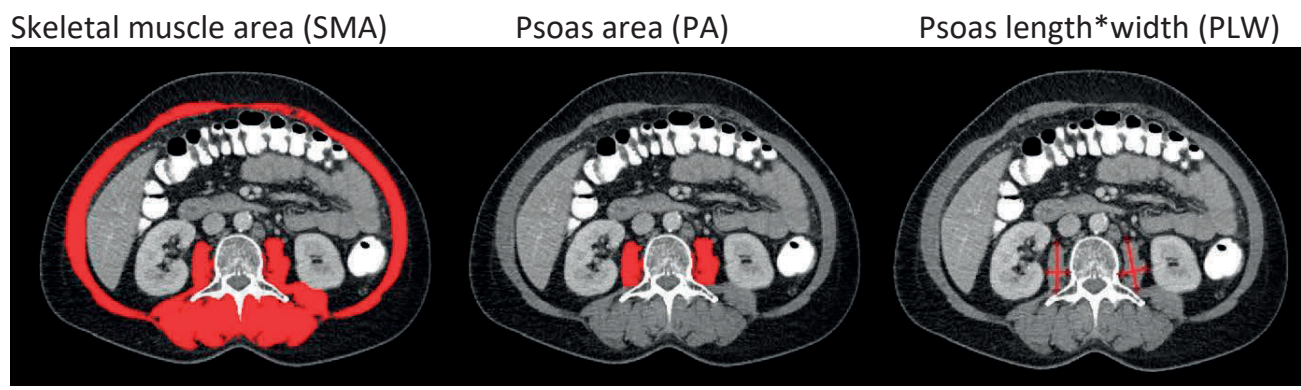

Afterwards, measurements were averaged between the observers. Mean assessment time was recorded for a random sample of patients $(n=10)$ for each method. This assessment time only included the time in which the actual measurements were performed after the correct axial image at the level of $\mathrm{L} 3$ had already been selected and the right $\mathrm{HU}$ boundaries had been defined.

\section{Statistical analysis}

Interobserver correlation between observer 1 and 2 was evaluated for each method with the Pearson correlation coefficient $(r)$ and with the intraclass correlation coefficient (ICC). Pearson's $r$ measures linear correlation between two variables and equals 1 in case of perfect positive correlation, 0 in case of no correlation at all, and -1 in case of total negative correlation. ICC can be used to assess both consistency and absolute agreement between quantitative measurements made by multiple observers measuring the same quantity. Further statistical analyses were conducted with the averaged measurements between observers. Intermeasurement correlations between the three methods to measure muscle tissue were also studied with the Pearson correlation coefficient. Additionally a Bland-Altman plot was created to investigate the existence of any systematic differences between the two assay methods measuring psoas muscle area; PA and PLW. If the mean value of the difference between assay methods is significantly different from zero, this indicates a systematic difference between measurements.

The percentage change in muscle area between the pre- and post-chemotherapy CT scan was calculated per 100 days for each method. A measurement error of $2 \%$ was taken into account based on previously reported accuracy of CT for muscle analysis [9]. Patients were divided into two groups based on the extent of muscle area change: "Loss" in case of $>2 \%$ decrease per 100 days and "Gain" when any increase or $\leq 2 \%$ decrease was seen. Muscle area changes between $-2 \%$ and $+2 \%$ were considered as muscle stability and were included in the "Gain" group. Subsequently the three methods to quantify muscle area were compared categorically through the use of contingency tables. Cohen's Kappa coefficient $(\mathrm{K})$ was computed to measure agreement between SMA and PA, SMA and PLW, and PA and PLW. In case of perfect agreement, $\mathrm{k}$ gives a value of 1 .

Finally age, FIGO stage ("FIGO stage IV" versus "FIGO stage II and III combined"), surgical outcome ("complete" versus "incomplete and optimal combined") and muscle change determined by SMA, PA and PLW ("loss" versus "gain") were tested as effect modifiers in a univariable proportional hazards Cox-regression model at a significance level of $10 \%$. Hazard ratio's (HR) were calculated with $95 \%$ confidence intervals $(95 \% \mathrm{Cl})$. Using backwards stepwise elimination significant predictors were combined in a multivariable model in which a 
significance level of $5 \%$ was applied. All analyses were performed with the statistical software package SPSS v20.0 (IBM Corp, Chicago, IL).

\section{Results}

\section{Baseline characteristics}

In total, 190 patients with advanced ovarian cancer treated with induction chemotherapy and interval debulking were identified. 40 patients were excluded due to unavailability or insufficient quality of one or both CT scans, due to missing clinical data or due to debulking being performed for recurrent disease. After exclusion, 150 patients and $300 \mathrm{CT}$ scans were available for analysis. 123 of these 150 patients were used in previous investigations by our research group [4]. Patient characteristics are presented in Table 1.

Table 1 Patient Characteristics

\begin{tabular}{|c|c|}
\hline & All patients $(n=150)$ \\
\hline Age in years, median \pm SD (range) & $67 \pm 9.8(39-86)$ \\
\hline$\leq 60$ years, $\mathrm{n}(\%)$ & $40(26.7)$ \\
\hline $61-70$ years, $n(\%)$ & $51(34.0)$ \\
\hline$>70$ years, $n(\%)$ & $59(39.3)$ \\
\hline \multicolumn{2}{|l|}{ FIGO tumour stage } \\
\hline II, n (\%) & $2(1.3)$ \\
\hline III, n (\%) & $91(60.7)$ \\
\hline IV, n (\%) & $57(38.0)$ \\
\hline $\begin{array}{l}\text { Days between } \mathrm{CT} \text { scans, median } \pm \mathrm{SD} \\
\text { (range) }\end{array}$ & $82.5 \pm 22.4(47-190)$ \\
\hline \multicolumn{2}{|l|}{$S M A$ in $\mathrm{cm}^{2}$} \\
\hline Pre-chemotherapy, median \pm SD & $110.2 \pm 15.4$ \\
\hline Post-chemotherapy, median \pm SD & $104.4 \pm 14.3$ \\
\hline \multicolumn{2}{|l|}{$P A$ in $\mathrm{cm}^{2}$} \\
\hline Pre-chemotherapy, median \pm SD & $13.3 \pm 3.1$ \\
\hline Post-chemotherapy, median \pm SD & $13.4 \pm 2.9$ \\
\hline \multicolumn{2}{|l|}{ PLW in $\mathrm{cm}^{2}$} \\
\hline Pre-chemotherapy, median \pm SD & $14.9 \pm 4.3$ \\
\hline Post-chemotherapy, median \pm SD & $14.5 \pm 4.0$ \\
\hline \multicolumn{2}{|l|}{$\begin{array}{l}\text { Muscle area changes in \% change per } \\
100 \text { days }\end{array}$} \\
\hline $\mathrm{SMA}$, median $\pm \mathrm{SD}$ & $-5.8 \pm 9.9$ \\
\hline PA, median \pm SD & $+1.4 \pm 21.1$ \\
\hline PLW, median \pm SD & $-0.9 \pm 15.7$ \\
\hline \multicolumn{2}{|l|}{ Outcome interval debulking } \\
\hline Complete, n (\%) & $69(46.0)$ \\
\hline Optimal, n (\%) & $55(36.7)$ \\
\hline Incomplete, n (\%) & $26(17.3)$ \\
\hline OS in days, median \pm SD & $711 \pm 753$ \\
\hline
\end{tabular}

SD, standard deviation; FIGO, International Federation of Gynaecology and Obstetrics; SMA, skeletal muscle area; PA, psoas area; PLW, psoas length*width; OS, overall survival 
Median follow-up for censored patients was 904 days $(n=56)$ with a minimum of 209 days. Specification of muscle area change resulted in a median SMA loss of 5.8\% per 100 days, a median PA gain of $1.4 \%$ per 100 days and a median PLW loss of $0.9 \%$ per 100 days. Mean assessment time was 110 seconds per patient, 27 seconds per patient and 16 seconds per patient for SMA, PA and PLW respectively.

\section{Interobserver and intermeasurement correlations}

Interobserver correlation results for the different measurements on the pre-chemotherapy CT scan are given in Figure 2. For assessment of SMA and PA, agreement was almost perfect with Pearson's $r$ values of 0.96 and 0.99 respectively. Interobserver agreement for PLW was 0.85 . Mean SM measured by observer 1 was 110.7 with a standard deviaton (SD) of 15.4 and mean SM measured by observer 2 was 112.2, SD 15.8. Mean PA was 13.7 (SD 3.2) for observer 1 and 13.9 (SD 3.1) for observer 2. Mean PLW was 15.9 (SD 4.6) for observer 1 and 14.4 (SD 4.3) for observer 2. ICC assessing consistency and absolute agreement between observers was 0.96 and 0.96 for SM, 0.99 and 0.99 for PA and 0.85 and 0.80 for PLW respectively. Interobserver agreement was also measured for post chemotherapy scans which resulted in similar Pearson's $r$ values of $0.99,0.98$ and 0.80 for SMA, PA and PLW respectively.

Figure 2 Interobserver correlation, analysis with data from pre-chemotherapy CT scan

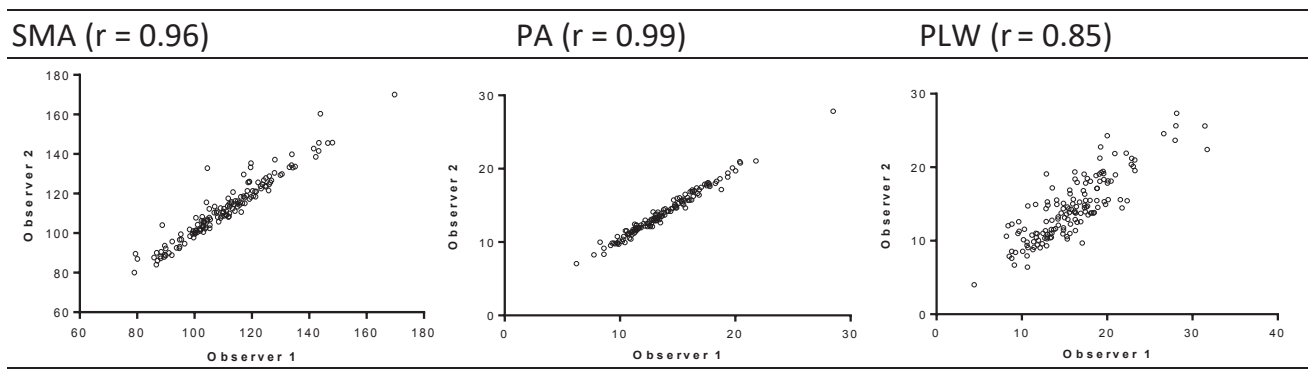

SMA, skeletal muscle area; PA, psoas area; PLW, psoas length*width

Intermeasurement correlation between SMA and PA was 0.52 and 0.56 for pre- and postchemotherapy scans respectively. Correlation between SMA and PLW was 0.39 and 0.44 for pre- and post-chemotherapy scans respectively. Correlation between PA and PLW was 0.83 and 0.84 for pre- and post-chemotherapy scans respectively. Scatter plots for correlation between the methods applied to the pre-chemotherapy scan are shown in Figure 3 . The mean difference between PLW and PA measuring psoas muscle on the pre-chemotherapy scan was 1.35 with a standard deviation of 2.33 , which was significantly different from zero $(p<0.001)$ and indicates that the two assay methods are systematically producing different results. The corresponding Bland-Altman plot created with 95\% confidence intervals is shown in Figure 4. 
Figure 3 Intermeasurement correlation, analysis with data from pre-chemotherapy CT scan

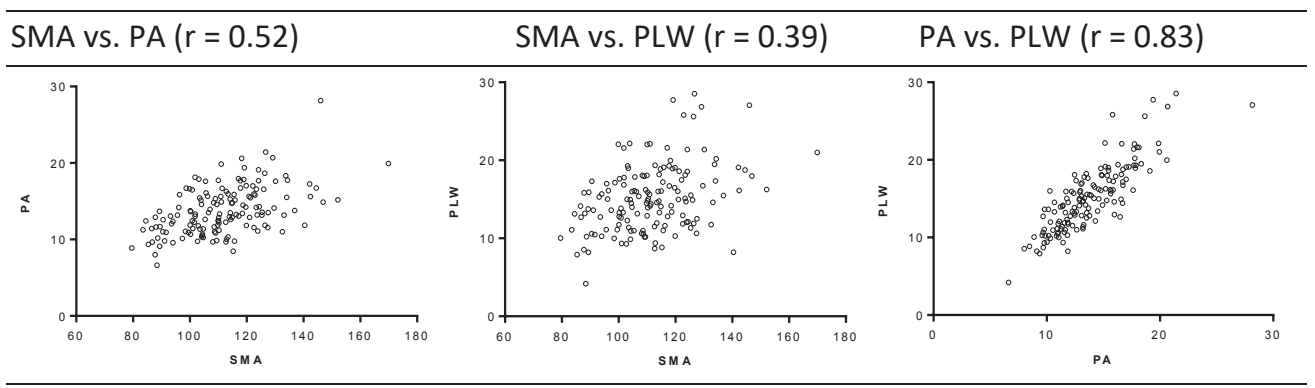

SMA, skeletal muscle area; PA, psoas area; PLW, psoas length*width

Contingency tables in which patients were categorised into loss or gain are shown in Table 2 . Cohen's K was 0.182 for SMA and PA, 0.312 for SMA and PLW and 0.226 for PA and PLW. Discrepancies were found in $44 \%(n=66)$ of cases when comparing SMA and PA. In the comparison of SMA and PLW, 35\% $(n=53)$ discrepant cases were found and PA and PLW disagreed in $38 \%(n=57)$ of cases.

Table 2 Contingency tables

SMA vs. PA ( $k=0.182) \quad$ SMA vs. PLW $(k=0.312) \quad$ PA vs. PLW $(k=0.226)$

\begin{tabular}{|c|c|c|c|c|c|c|c|c|c|c|c|c|c|c|}
\hline \multirow{5}{*}{$\sum_{n}^{\nwarrow}$} & \multicolumn{4}{|c|}{ PA } & & \multicolumn{4}{|c|}{ PLW } & & \multicolumn{4}{|c|}{ PLW } \\
\hline & & Loss & Gain & Total & \multirow{4}{*}{$\sum_{n}^{\mathbb{S}}$} & & Loss & Gain & Total & \multirow{4}{*}{ 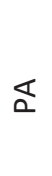 } & & Loss & Gain & Total \\
\hline & Loss & 46 & 54 & 100 & & Loss & 58 & 42 & 100 & & Loss & 35 & 23 & 58 \\
\hline & Gain & 12 & 38 & 50 & & Gain & 11 & 39 & 50 & & Gain & 34 & 58 & 92 \\
\hline & Total & 58 & 92 & 150 & & Total & 69 & 81 & 150 & & Total & 69 & 81 & 150 \\
\hline
\end{tabular}

SMA, skeletal muscle area; PA, psoas area; PLW, psoas length*width;

\section{Survival analysis}

Age, FIGO stage IV, complete interval debulking and muscle loss measured with SMA were significant predictors of OS at a significance level of $10 \%$ in univariable Cox-regression analysis (Table 3). No relationship with OS was seen for measurement of psoas muscle loss with PA nor with PLW. In multivariable analysis FIGO stage IV (HR $1.730(95 \% \mathrm{Cl} 1.129-2.652), \mathrm{p}=0.012)$, complete interval debulking (HR $0.381(95 \% \mathrm{Cl} 0.246-0.589), \mathrm{p}<0.001)$ and loss of SMA (HR $1.698(95 \% \mathrm{Cl} 1.038-2.778), p=0.035)$ were predictive of OS. Median OS was 665 days for patients who lost SMA compared to 914 days for patients who maintained or gained SMA $(p=0.017)$. 
Table 3 Univariable and multivariable Cox-regression analysis

\begin{tabular}{lllll}
\hline \multirow{2}{*}{ Variables } & \multicolumn{2}{l}{ Univariable analysis } & \multicolumn{2}{c}{ Multivariable analysis } \\
\cline { 2 - 5 } Age & $\mathrm{HR}(95 \% \mathrm{Cl})$ & $\mathrm{p}$-value & $\mathrm{HR}(95 \% \mathrm{Cl})$ & $\mathrm{p}$-value \\
\hline FIGO tumour stage IV & $1.026(1.002-1.050)$ & $0.032^{*}$ & - & - \\
Complete interval debulking & $0.408(0.259-0.643)$ & $<0.001^{*}$ & $0.381(0.246-0.589)$ & $<0.001^{*}$ \\
Muscle loss - SMA & $2.069(1.285-3.332)$ & $0.003^{*}$ & $1.698(1.038-2.778)$ & $0.035^{*}$ \\
Muscle loss - PA & $0.979(0.643-1.491)$ & 0.921 & - & - \\
Muscle loss - PLW & $1.101(0.730-1.662)$ & 0.645 & - & - \\
\hline HR, hazard ratio; Cl, confidence interval; FIGO, International Federation of Gynaecology and \\
Obstetrics; SMA, skeletal muscle area; PA, psoas area; PLW, psoas length*width. \\
* Indicates significant p-value $(\mathrm{p}<0.10$ for univariable analysis and p<0.05 for multivariable \\
analysis).
\end{tabular}

\section{Discussion}

\section{Key findings}

The objective of this study was to investigate whether assessment of psoas muscle area reflects total muscle area and can be used to assess sarcopenia in ovarian cancer patients undergoing induction chemotherapy and interval debulking. Two different quantification methods of the psoas muscle area were compared to the reference method of measuring total skeletal muscle area. We found a weak correlation between SMA and PA measurements and an even weaker correlation between SMA and PLW. When categorising the findings into muscle area loss or gain, high rates of discrepancies were also found between both SMA and PA or PLW. The correlation between the two assessment methods of psoas area was reasonable but also resulted in a high number of discrepancies when categorising patients. From these data we can conclude that measurement of psoas muscle area either with software delineation of surface area or with manual measurement of length and width does not give a reliable representation of skeletal muscle loss when compared to measurement of total skeletal muscle mass. The regression analysis confirmed these results; PA and PLW were not helpful in the prediction of OS whereas SMA proved to be an important individual factor in both univariable and multivariable Cox regression-analysis corrected for age, tumour stage and surgical outcome. A clear difference in median OS was seen between patients who lost SMA and patients who maintained or gained SMA (665 days versus 914 days).

Many studies have used PA instead of SMA for assessment of sarcopenia in various cancer types. Although in some studies a correlation between PA and post-operative complications was seen, the majority have failed to prove a relationship between PA-assessed sarcopenia and survival [11-23]. Only few have actually assessed the agreement between PA and SMA within their population; Jones et al. studied 100 patients with colorectal cancer and reported a Spearman correlation of 0.8 for PA and SMA and a Spearman correlation of 0.94 for PA and PLW, which could not be reproduced in our cohort [11]. A possible explanation for this discrepancy might be the difference in statistical analysis. Whereas we used the Pearson correlation coefficient, Jones et al. used Spearman's rank correlation coefficient. Pearson's method is used for linear relationships and was applicable to our data while Spearman's method can be applied to nonparametric data. The outcome produced by the two methods 
can vary according to the character of the data. It is unclear why the authors chose to use Spearman instead of Pearson correlation. However, when we tested Spearman's correlation in our data, results were not different from what we found with Pearson's correlation and they were again not comparable to the high correlation found in Jones' study. Another plausible difference between our studies is the software that was used: ImageJ versus SliceOmatic. However since both software programs measure and quantify tissue by outlining the muscle area and similar measurements are expected by both methods, this does not fully explain the difference in intermeasurement correlation. In addition to the former study, Taguchi et al. found a Spearman correlation of 0.75 for PA and SMA in 64 patients with urothelial carcinoma which was also markedly higher than the Pearson correlation of 0.52 we found in our population [30]. In this study a slightly different method of assessing PLW was used in which the length and width of the psoas muscle were compared separately and not as a combined measure. The reported Spearman correlation of 0.81 for PA and psoas width was comparable to our reported Pearson correlation of 0.83 for PA and PLW.

Figure 4 Bland-Altman plot comparing PLW and PA for measurement of psoas muscle area, analysis with data from pre-chemotherapy CT scan.

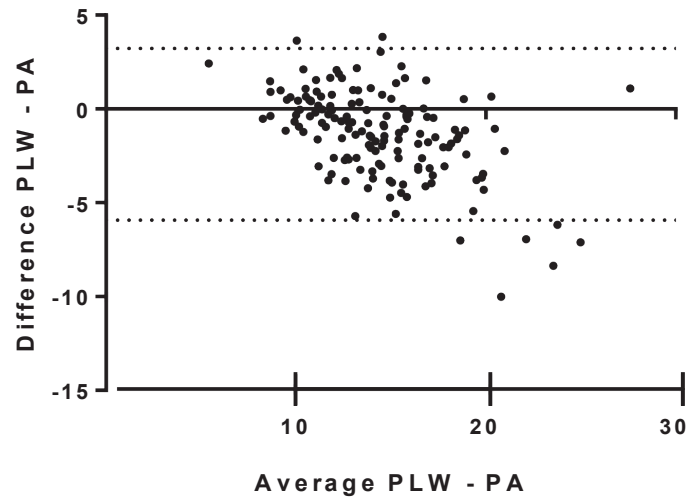

PLW, psoas length*width; PA, psoas area 
We believe that L3 psoas muscle area measurements are not representative of total L3 skeletal muscle area. A plausible reason why the psoas muscle is less representative than the total muscle at L3 is that the psoas muscle is prone to be focally affected by degenerative diseases of the lumbar spine. Lumbar degenerative disc and facet joint disease can cause local atrophy of the trunk muscles and psoas muscle loss is hence not specifically related to cancerinduced sarcopenia [31]. Psoas analysis should therefore not be conducted on patients with a medical history of spine surgery, lower back pain, degenerative lumbar instability, vertebral fracture, and deformity. This has a massive impact when studying cancer populations. First, medical records of all patients need to be evaluated to confirm which individuals are affected by the above conditions and, subsequently, will have to be excluded from the analysis. Secondly, degenerative diseases of the lumbar spine are rather prevalent in older patients, and in cancer populations (the mean age in our study population was $>65$ years). This would result in the psoas method being inapplicable in large numbers of individuals and especially elderly. Although the psoas muscle is a hip flexor muscle which could be expected to give a representation of physical fitness, the PA only represents $10 \%$ of the SMA measurable at L3. By using the PA alone you ignore vital information about the remaining skeletal muscles. Because the PA is so much smaller in comparison to the SMA, it is also much less sensitive to depict change. In our opinion, measuring change over time is the most accurate way to portray sarcopenia in ovarian cancer and therefore we believe that PA should not be used to substitute SMA to predict survival. The rates of muscle loss and gain we found seem to be consistent throughout the 15 -year period of time that we studied since we have not noticed specific outliers within a certain time period.

Our results have shown an exceedingly well interobserver agreement with correlation coefficients between 0.96 and 0.99 for assessment of SMA and PA with SliceOmatic software. When comparing the results for Pearson's correlation and intraclass correlation we find almost identical results, which is suggestive for both a high consistency and high agreement between observers. A similar interobserver agreement $(r=0.97)$ was found by Jones et al. for the evaluation of PA [11]. Agreement between observers was less strong for the manual technique measuring PLW ( $r=0.80-0.85)$. This may be explained by the fact that the length and width of the psoas muscle are difficult to measure due to the great variation in shape of the psoas muscle. The psoas muscle is only a small muscle; therefore any small errors in measurement may extrapolate to larger errors in rate of change when multiplying length and width in PLW measurements, contributing to a lower interobserver agreement. Additionally, when assessing psoas muscle with PLW, the intramuscular adipose tissue is included in the measurement which gives an overestimation of the actual muscle tissue. In the pathophysiology of (cancer) cachexia, skeletal muscle may be replaced by intramuscular adipose tissue. In this way, both the quantity and the quality of muscle is being influenced. Linear methods of assessing skeletal muscle such as PLW measurements cannot depict these important changes. Software delineation using predefined HU boundaries can take the intramuscular adipose tissue into account and therefore reflects a more accurate quantity of skeletal muscle. The overestimation of muscle surface area with a linear method is reflected in Table 1 where we find a higher mean PLW in comparison to mean PA.

\section{Limitations}

Due to the retrospective nature of this research our analyses were to some extent restricted by missing data. Due to several irretrievable CT scans, a number of patients were excluded from the analysis. Also, by limiting our study population to patients who were treated with 
induction chemotherapy and interval debulking and excluding patients treated with primary debulking we have created a selection bias. Effects on muscle mass might become more apparent in the population treated with induction chemotherapy which is prone to have more advanced tumour spread and/or a worse performance status. Whether evaluation of PA or muscle area estimation in general has any importance in ovarian cancer patients who receive primary debulking surgery without induction chemotherapy is unclear. Previous studies in other cancer populations have mainly focused on the relationship between PA and surgical complications and used only one CT measurement. Ovarian cancer patients selected for primary surgical treatment are also subjected to only one clinical CT scan and it would be interesting to see if an association can be found between PA and complications in this group.

Part of our study population was used in previous investigations from which the results have been published recently [4]. In this manuscript we concluded that SMA loss was predictive of OS, which was confirmed in the current study. However the additional value of this finding may be limited due to the overlap in patients studied ( $82 \%$ overlap). External validation of these findings in patients with other gynaecological malignancies and ovarian cancer specifically is imperative. Comparable results have been reported for patients with lung cancer, pancreatic cancer and colorectal cancer but these populations were primarily comprised of male patients and translation of these results to female patients - with lower muscle mass in general - should be applied carefully [6-8].

\section{Conclusion and implications for practice and research}

Change in psoas muscle area is not representative of total muscle area change and should not be used to substitute total skeletal muscle to predict survival in patients with ovarian cancer undergoing induction chemotherapy and interval debulking. Assessment of psoas muscle area may be easier and quicker, but is less sensitive to muscle change than standard assessment of total skeletal muscle. Measuring cross-sectional total skeletal muscle area at L3 showed strong interobserver agreement and has proven to be a significant predictor for OS and should therefore not be substituted by psoas area evaluation alone.

This study does not answer the important question why some patients with ovarian cancer lose while others gain muscle mass. The present study underpins the observation that sarcopenia is a problem in patients with ovarian cancer and that it has a substantial effect on survival. External validation of our findings is crucial and may lead to prospective intervention trials investigating how prevention of muscle loss can improve prognosis of patients with ovarian cancer. 


\section{References}

1. Surveillance E, and End Results (SEER) Program SEER 9 Incidence \& U.S. Mortality Research Data (1973-2011). In: National Cancer Institute D, Surveillance Research Program, Surveillance Systems Branch, released April 2014, based on the November 2013 submission, (ed),

2. du Bois A, Reuss A, Pujade-Lauraine E, Harter P, Ray-Coquard I, Pfisterer J (2009) Role of surgical outcome as prognostic factor in advanced epithelial ovarian cancer: a combined exploratory analysis of 3 prospectively randomized phase 3 multicenter trials: by the Arbeitsgemeinschaft Gynaekologische Onkologie Studiengruppe Ovarialkarzinom (AGOOVAR) and the Groupe d'Investigateurs Nationaux Pour les Etudes des Cancers de l'Ovaire (GINECO). Cancer 115:1234-1244

3. Vergote I, Trope CG, Amant F et al (2010) Neoadjuvant chemotherapy or primary surgery in stage IIIC or IV ovarian cancer. N Engl J Med 363:943-953

4. Rutten IJG, Van Dijk DPJ, Kruitwagen RFPM, Beets-Tan RGH, Olde Damink SW, Van Gorp T (2016) Loss of skeletal muscle during neoadjuvant chemotherapy is related to decreased survival in ovarian cancer patients. Journal of Cachexia, Sarcopenia and Muscle. 10.1002/jcsm.12107

5. Aust S, Knogler T, Pils D et al (2015) Skeletal Muscle Depletion and Markers for Cancer Cachexia Are Strong Prognostic Factors in Epithelial Ovarian Cancer. PLoS One 10:e0140403

6. Choi Y, Oh DY, Kim TY et al (2015) Skeletal Muscle Depletion Predicts the Prognosis of Patients with Advanced Pancreatic Cancer Undergoing Palliative Chemotherapy, Independent of Body Mass Index. PLoS One 10:e0139749

7. Miyamoto Y, Baba Y, Sakamoto $Y$ et al (2015) Negative Impact of Skeletal Muscle Loss after Systemic Chemotherapy in Patients with Unresectable Colorectal Cancer. PLoS One 10:e0129742

8. Stene GB, Helbostad JL, Amundsen T et al (2015) Changes in skeletal muscle mass during palliative chemotherapy in patients with advanced lung cancer. Acta Oncol 54:340-348

9. Mourtzakis M, Prado CM, Lieffers JR, Reiman T, McCargar LJ, Baracos VE (2008) A practical and precise approach to quantification of body composition in cancer patients using computed tomography images acquired during routine care. Appl Physiol Nutr Metab 33:997-1006

10. Shen W, Punyanitya M, Wang Z et al (2004) Total body skeletal muscle and adipose tissue volumes: estimation from a single abdominal cross-sectional image. J Appl Physiol (1985) 97:2333-2338

11. Jones KI, Doleman B, Scott S, Lund JN, Williams JP (2015) Simple psoas cross-sectional area measurement is a quick and easy method to assess sarcopenia and predicts major surgical complications. Colorectal Dis 17:020-26

12. Peng PD, van Vledder MG, Tsai $S$ et al (2011) Sarcopenia negatively impacts short-term outcomes in patients undergoing hepatic resection for colorectal liver metastasis. HPB (Oxford) 13:439-446

13. Peyton CC, Heavner MG, Rague JT, Krane LS, Hemal AK (2016) Does Sarcopenia Impact Complications and Overall Survival in Patients Undergoing Radical Nephrectomy for Stage III and IV Kidney Cancer? J Endourol 30:229-236

14. Smith AB, Deal AM, Yu H et al (2014) Sarcopenia as a predictor of complications and survival following radical cystectomy. J Urol 191:1714-1720

15. Valero V, 3rd, Amini N, Spolverato G et al (2015) Sarcopenia adversely impacts postoperative complications following resection or transplantation in patients with primary liver tumors. $J$ Gastrointest Surg 19:272-281

16. Otsuji H, Yokoyama Y, Ebata T et al (2015) Preoperative sarcopenia negatively impacts postoperative outcomes following major hepatectomy with extrahepatic bile duct resection. World J Surg 39:1494-1500

17. Amini N, Spolverato G, Gupta R et al (2015) Impact Total Psoas Volume on Short- and LongTerm Outcomes in Patients Undergoing Curative Resection for Pancreatic Adenocarcinoma: a New Tool to Assess Sarcopenia. J Gastrointest Surg 19:1593-1602

18. Kuroki LM, Mangano M, Allsworth JE et al (2015) Pre-operative Assessment of Muscle Mass to Predict Surgical Complications and Prognosis in Patients With Endometrial Cancer. Ann Surg Oncol 22:972-979 
19. Okumura S, Kaido T, Hamaguchi Y et al (2015) Impact of the preoperative quantity and quality of skeletal muscle on outcomes after resection of extrahepatic biliary malignancies. Surgery. 10.1016/j.surg.2015.08.047

20. Wilson RJ, Alamanda VK, Hartley KG et al (2015) Sarcopenia Does Not Affect Survival or Outcomes in Soft-Tissue Sarcoma. Sarcoma 2015:146481

21. Okumura S, Kaido T, Hamaguchi $Y$ et al (2015) Impact of preoperative quality as well as quantity of skeletal muscle on survival after resection of pancreatic cancer. Surgery 157:1088-1098

22. Jung HW, Kim JW, Kim JY et al (2015) Effect of muscle mass on toxicity and survival in patients with colon cancer undergoing adjuvant chemotherapy. Support Care Cancer 23:687-694

23. Miller BS, Ignatoski KM, Daignault $S$ et al (2012) Worsening central sarcopenia and increasing intra-abdominal fat correlate with decreased survival in patients with adrenocortical carcinoma. World J Surg 36:1509-1516

24. Fujikawa H, Araki T, Okita $Y$ et al (2016) Impact of sarcopenia on surgical site infection after restorative proctocolectomy for ulcerative colitis. Surg Today. 10.1007/s00595-016-1357-x

25. Masuda T, Shirabe K, Ikegami T et al (2014) Sarcopenia is a prognostic factor in living donor liver transplantation. Liver Transpl 20:401-407

26. Zakaria HM, Schultz L, Mossa-Basha F, Griffith B, Chang V (2015) Morphometrics as a predictor of perioperative morbidity after lumbar spine surgery. Neurosurg Focus 39:E5

27. Mamane S, Mullie L, Piazza N et al (2016) Psoas Muscle Area and All-Cause Mortality After Transcatheter Aortic Valve Replacement: The Montreal-Munich Study. Can J Cardiol 32:177182

28. Onesti JK, Wright GP, Kenning SE et al (2016) Sarcopenia and survival in patients undergoing pancreatic resection. Pancreatology 16:284-289

29. Saji M, Lim DS, Ragosta M et al (2016) Usefulness of Psoas Muscle Area to Predict Mortality in Patients Undergoing Transcatheter Aortic Valve Replacement. Am J Cardiol 118:251-257

30. Taguchi S, Akamatsu N, Nakagawa T et al (2015) Sarcopenia Evaluated Using the Skeletal Muscle Index Is a Significant Prognostic Factor for Metastatic Urothelial Carcinoma. Clin Genitourin Cancer. 10.1016/j.clgc.2015.07.015

31. Sebro R, O'Brien L, Torriani M, Bredella MA (2016) Assessment of trunk muscle density using $\mathrm{CT}$ and its association with degenerative disc and facet joint disease of the lumbar spine. Skeletal Radiol 45:1221-1226 


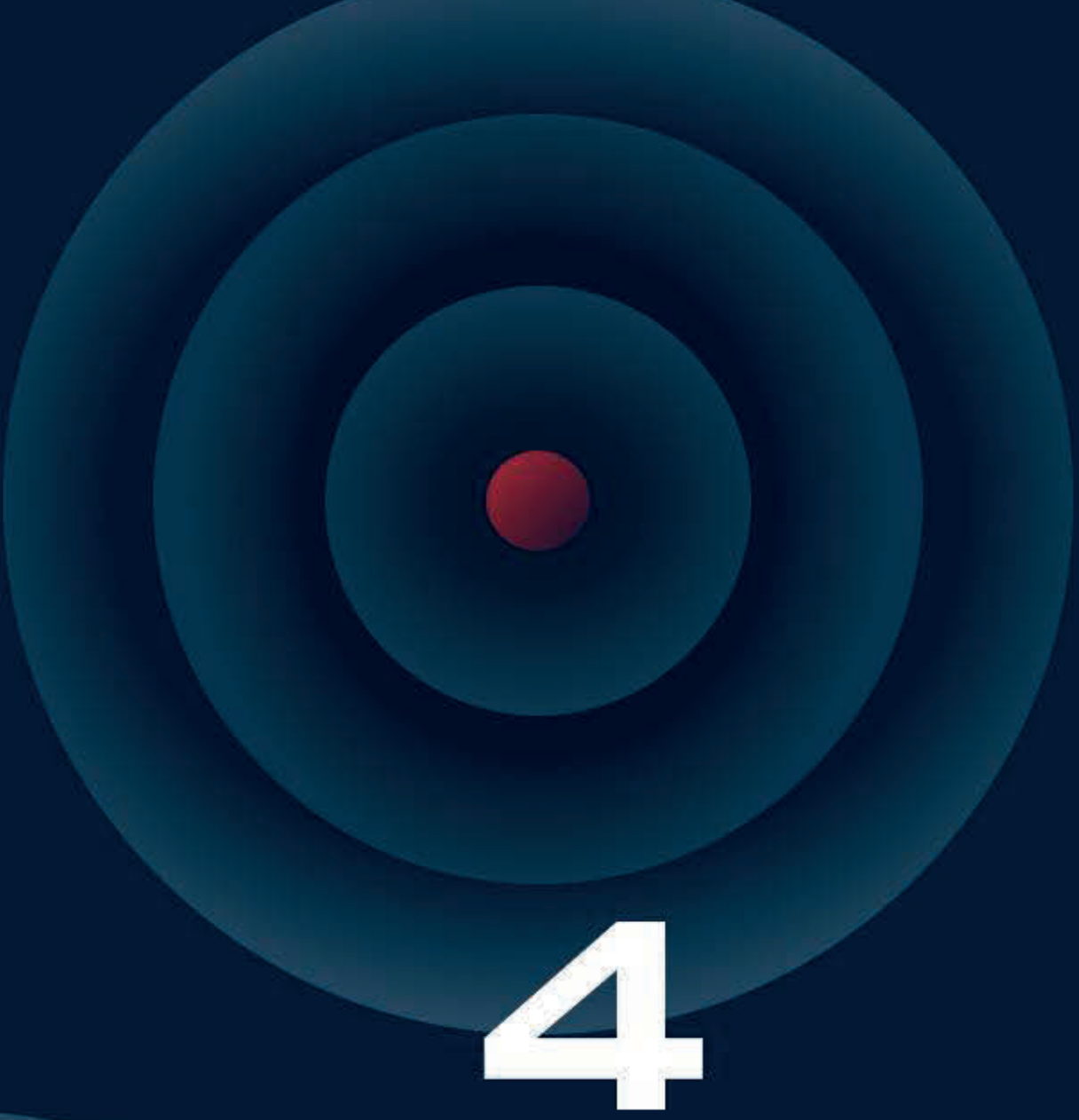


The influence of sarcopenia on survival and surgical complications in ovarian cancer patients undergoing primary debulking surgery

European Journal of Surgical Oncology (2017)

Jorne Ubachs, Iris Rutten, Roy Kruitwagen, David van Dijk, Regina Beets-Tan, Leon Massuger, Steven Olde Damink, Toon Van Gorp. 


\begin{abstract}
Background

Sarcopenia, severe skeletal muscle loss, has been identified as a prognostic factor in various malignancies. This study aims to investigate whether sarcopenia is associated with overall survival (OS) and surgical complications in patients with advanced ovarian cancer undergoing primary debulking surgery (PDS).
\end{abstract}

\title{
Methods
}

Ovarian cancer patients $(n=216)$ treated with PDS were enrolled retrospectively. Total skeletal muscle surface area was measured on axial computed tomography at the level of the third lumbar vertebra. Optimum stratification was used to find the optimal skeletal muscle index cut-off to define sarcopenia $\left(\leq 38.73 \mathrm{~cm}^{2} / \mathrm{m}^{2}\right)$. Cox-regression and Kaplan-Meier analysis were used to analyse the relationship between sarcopenia and OS. The effect of sarcopenia on the development of major surgical complications was studied with logistic regression.

\section{Results}

Kaplan-Meier analysis showed a significant survival disadvantage for patients with sarcopenia compared to patients without sarcopenia $(p=0.010)$. Sarcopenia univariably predicted OS (HR $1.536(95 \% \mathrm{Cl} 1.105-2.134), \mathrm{p}=0.011)$ but was not significant in multivariable Cox-regression analysis (HR $1.362(95 \% \mathrm{Cl} 0.968-1.916) \mathrm{p}=0.076)$. Significant predictors for OS in multivariable Cox-regression analysis were complete PDS, treatment in a specialised centre and the development of major complications. Sarcopenia was not predictive of major complications.

\section{Conclusion}

Sarcopenia was not predictive of OS or major complications in ovarian cancer patients undergoing primary debulking surgery. However a strong trend towards a survival disadvantage for patients with sarcopenia was seen. Future prospective studies should focus on interventions to prevent or reverse sarcopenia and possibly increase ovarian cancer survival. Complete cytoreduction remains the strongest predictor of ovarian cancer survival. 


\section{Introduction}

Ovarian cancer is the fifth leading cause of cancer-related death among women in developed countries and claims over 150.000 lives worldwide yearly [1]. The majority of patients have abdominally metastasized disease at first presentation which resembles a 5-year survival of 17-36\% [2]. Preferred treatment for patients with advanced FIGO stage (International Federation of Gynecology and Obstetrics) ovarian cancer is upfront primary debulking surgery (PDS). For patients who are considered inoperable or have estimated irresectable disease, neoadjuvant chemotherapy complemented with interval debulking surgery (IDS) provides a legitimate alternative [3]. To remove all visible tumour at surgery is pivotal as the amount of residual disease after surgery is the most influential prognostic factor contributing to ovarian cancer survival along with FIGO stage, tumour grade and performance status [4]. Additionally women with ovarian cancer often experience cancer cachexia characterised by involuntary weight loss and severe muscle wasting which can further abate their survival chances [5]. The mechanisms behind cancer cachexia and muscle depletion involve an interplay between reduced nutrient intake and abnormal metabolism incited by an excessive systemic inflammation and catabolic (tumour-related) mediators to which the host (i.e. the patient) is unable to respond properly [6]. However, cancer-related weight loss can be disguised by bulky tumour deposits or ascites and is less reliable as a measure of disease activity in ovarian cancer patients. Instead of weight measurements, measurements of body composition are now extensively being used for prognostic purposes in cancer patients. Cross-sectional computed tomography (CT) scans at the level of the third lumbar vertebra (L3) give an accurate representation of total body adipose and skeletal muscle tissue [7-9]. Severe loss of muscle mass (i.e. sarcopenia) as estimated on CT was henceforth identified as a risk factor for survival in patients with various malignancies [10-13]. In a cohort of ovarian cancer patients undergoing neoadjuvant chemotherapy and IDS, loss of skeletal muscle over time was also related to decreased survival [14]. However two earlier studies could not find a relationship between sarcopenia and survival when evaluating ovarian cancer patients treated with PDS $[15 ; 16]$. The association between sarcopenia and postoperative complications is thus far unknown in ovarian cancer but has shown significance in gastro-intestinal cancer [17; 18].

The data on ovarian cancer patients are scarce but there is evidence that sarcopenia could be one of the few modifiable risk factors for survival if patients with sarcopenia were to be identified timely. The reversal of sarcopenia with nutritional supplements and physical activity has been studied successfully in sarcopenic elderly [19]. Exercise might play an important role in counteracting muscle wasting through reducing the level of inflammation, increasing insulin sensitivity and modulation of muscle protein metabolism [20; 21]. Although there is no direct evidence that anti-sarcopenia treatment increases survival chances in patients with advanced malignancies there is a rationale for implementing e.g. exercise therapy in oncologic patients with sarcopenia [22]. The primary objective of this study is to evaluate whether sarcopenia is associated with survival and the development of major postoperative complications in patients with advanced ovarian cancer undergoing PDS.

\section{Patients and methods}

This study has been performed in accordance with the ethical standards laid down in the 1964 Declaration of Helsinki. The study protocol has been approved by the Maastricht University Medical Centre ethics committee which waived the requirement to obtain informed consent. 


\begin{tabular}{|c|c|c|c|}
\hline & $\begin{array}{l}\text { All patients } \\
(n=216)\end{array}$ & $\begin{array}{l}\text { Sarcopenia } \\
(n=70)\end{array}$ & $\begin{array}{l}\text { No } \\
\text { Sarcopenia } \\
(n=146)\end{array}$ \\
\hline \multicolumn{4}{|l|}{ Patient and tumour characteristics } \\
\hline Age in years, mean \pm SE (range) & $\begin{array}{l}63.1 \pm 0.8(16- \\
85)\end{array}$ & $\begin{array}{l}64.2 \pm 1.4 \\
(27-85)\end{array}$ & $\begin{array}{l}62.5 \pm 1.1 \\
(16-85)\end{array}$ \\
\hline Body Mass Index in $\mathrm{kg} / \mathrm{m}^{2}$, mean $\pm \mathrm{SE}$ & $24.9 \pm 0.3$ & $22.7 \pm 0.4^{a}$ & $26.0 \pm 0.3^{a}$ \\
\hline \multicolumn{4}{|l|}{ FIGO tumour stage, $\mathrm{n}(\%)$} \\
\hline II & $12(5.6)$ & $1(1.4)$ & $11(7.5)$ \\
\hline III & $161(74.5)$ & $50(71.4)$ & $111(76.0)$ \\
\hline IV & $43(19.9)$ & $19(27.1)$ & $24(16.4)$ \\
\hline \multicolumn{4}{|l|}{ Tumour grade, $\mathrm{n}(\%)$} \\
\hline 1 & $16(7.4)$ & $4(5.7)$ & $12(8.2)$ \\
\hline 2 & $47(21.8)$ & $12(17.1)$ & $35(24.0)$ \\
\hline 3 & $133(61.6)$ & $50(71.4)$ & $83(56.8)$ \\
\hline Unknown & $20(9.3)$ & $4(5.7)$ & $16(11.0)$ \\
\hline Presence of ascites, $\mathrm{n}(\%)$ & $157(72.7)$ & $61(87.1)^{a}$ & $96(65.8)^{a}$ \\
\hline \multicolumn{4}{|l|}{ Measurements } \\
\hline $\mathrm{SMI}$ in $\mathrm{cm}^{2} / \mathrm{m}^{2}$, mean $\pm \mathrm{SE}$ (cut-point) & $\begin{array}{l}41.99 \pm 0.39 \\
(38.73)\end{array}$ & $\begin{array}{l}35.73 \pm \\
0.28^{a}\end{array}$ & $44.98 \pm 0.34^{\mathrm{a}}$ \\
\hline $\begin{array}{l}\text { Psoas-index in } \mathrm{cm}^{2} / \mathrm{m}^{2} \text {, mean } \pm \mathrm{SE} \text { (cut- } \\
\text { point) }\end{array}$ & $\begin{array}{l}5.34 \pm 0.08 \\
(4.65)\end{array}$ & $4.64 \pm 0.10^{a}$ & $5.68 \pm 0.10^{\mathrm{a}}$ \\
\hline $\begin{array}{l}\text { IMAT-index in } \mathrm{cm}^{2} / \mathrm{m}^{2} \text {, mean } \pm \mathrm{SE} \text { (cut- } \\
\text { point) }\end{array}$ & $\begin{array}{l}5.28 \pm 0.22 \\
(3.51)\end{array}$ & $4.75 \pm 0.34$ & $5.54 \pm 0.28$ \\
\hline VAT-index in $\mathrm{cm}^{2} / \mathrm{m}^{2}$, mean $\pm \mathrm{SE}$ (cut-point) & $\begin{array}{l}27.11 \pm 1.45 \\
(13.22)\end{array}$ & $\begin{array}{l}19.68 \pm \\
1.87^{\mathrm{a}}\end{array}$ & $30.66 \pm 1.88^{\mathrm{a}}$ \\
\hline SAT-index in $\mathrm{cm}^{2} / \mathrm{m}^{2}$, mean $\pm \mathrm{SE}$ (cut-point) & $\begin{array}{l}62.40 \pm 2.29 \\
(44.52)\end{array}$ & $\begin{array}{l}47.65 \pm \\
2.84^{a}\end{array}$ & $69.47 \pm 2.94^{a}$ \\
\hline $\mathrm{MA}$ in $\mathrm{HU}$, mean $\pm \mathrm{SE}$ (cut-point) & $\begin{array}{l}36.64 \pm 0.62 \\
(33.67)\end{array}$ & $37.06 \pm 1.14$ & $36.43 \pm 0.73$ \\
\hline \multicolumn{4}{|l|}{ Surgical outcome } \\
\hline \multicolumn{4}{|l|}{ Outcome PDS, n (\%) } \\
\hline Complete & $70(32.4)$ & $18(25.7)$ & $52(35.6)$ \\
\hline Optimal & $51(23.6)$ & $18(25.7)$ & $33(22.6)$ \\
\hline Incomplete & $95(44.0)$ & $34(48.6)$ & $61(41.8)$ \\
\hline Blood loss in $\mathrm{mL}$, mean $\pm \mathrm{SE}$ & $1438 \pm 119$ & $1614 \pm 231$ & $1350 \pm 137$ \\
\hline Length of hospital stay in days, mean \pm SE & $14.2 \pm 0.8$ & $15.3 \pm 1.6$ & $13.6 \pm 0.9$ \\
\hline Re-admitted within 30 days, $\mathrm{n}(\%)$ & $13(6.0)$ & $5(7.5)$ & $8(5.7)$ \\
\hline \multicolumn{4}{|l|}{ Clavien-Dindo complications scale, $\mathrm{n}(\%)$} \\
\hline Grade 0 (No complications) & $121(56.0)$ & $36(51.4)$ & $85(58.2)$ \\
\hline $\begin{array}{l}\text { Grade I (Any deviation from normal } \\
\text { postoperative course) }\end{array}$ & $14(6.5)$ & $4(5.7)$ & $10(6.8)$ \\
\hline $\begin{array}{l}\text { Grade II (Requiring pharmacological } \\
\text { treatment) }\end{array}$ & $33(15.3)$ & $14(20.0)$ & $19(13.0)$ \\
\hline Grade III (Requiring invasive intervention) & $22(10.2)$ & $7(10.0)$ & $15(10.3)$ \\
\hline Grade IV (Life-threatening requiring ICU) & $15(6.9)$ & $6(8.6)$ & $9(6.2)$ \\
\hline
\end{tabular}




\begin{tabular}{llll}
$\begin{array}{lll}\text { Grade V (Death) } \\
\text { Unknown }\end{array}$ & $6(2.8)$ & $2(2.9)$ & $4(2.7)$ \\
Treatment, $\mathrm{n}(\%)$ & $5(2.3)$ & $1(1.4)$ & $4(2.7)$ \\
$\quad$ PDS & $154(71.3)$ & $47(67.1)$ & $107(73.3)$ \\
$\quad$ PDS + secondary IDS after chemotherapy & $62(28.7)$ & $23(32.9)$ & $39(26.7)$ \\
Treatment centre, $\mathrm{n}(\%)$ & & & \\
$\quad$ General care centre & $99(45.8)$ & $33(47.1)$ & $66(45.2)$ \\
$\quad$ Specialised oncologic centre & $117(54.2)$ & $37(52.9)$ & $80(54.8)$ \\
30-day mortality, $\mathrm{n}(\%)$ & $7(3.2)$ & $2(2.9)$ & $5(3.4)$ \\
OS in days, mean \pm SE & $1714 \pm 117$ & $1309 \pm 162^{\mathrm{a}}$ & $1887 \pm 147^{\mathrm{a}}$ \\
\hline
\end{tabular}

SE; standard error, FIGO; International Federation of Gynecology and Obstetrics, SMI; skeletal muscle index, IMAT; intramuscular adipose tissue, VAT; visceral adipose tissue, SAT; subcutaneous adipose tissue, MA; muscle radiation attenuation, HU; Hounsfield Units, PDS; primary debulking surgery, IDS; interval debulking surgery, ICU; intensive care

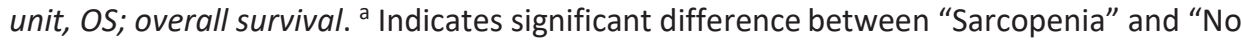
sarcopenia" ( $\mathrm{P}<0.05$, independent-samples T-test, chi-squared test or log rank test).

\section{Eligible patients}

All patients with advanced stage ovarian cancer (FIGO IIB-IV) treated with PDS between 2000 and 2015 were included in this retrospective cohort study. Patients underwent treatment in one of two specialised oncologic centres (Maastricht University or Radboud University medical centre) or in one of four general care centres in the Netherlands (Bernhoven, Rijnstate, Slingeland or St. Jansdal). The following inclusion criteria were applied: 1) an abdominal CTscan of sufficient quality taken within two months before PDS was available, 2) relevant clinical data were retrievable from the patient's medical records and 3) follow-up data were available for at least six months post-surgery.

Primary outcome was overall survival (OS), calculated as the time between surgery and death of any cause. Survivors were censored at a fixed date no sooner than six months after inclusion of the last patient. Postoperative complications were graded using the Clavien-Dindo scale of surgical complications [23]. We classified a Clavien-Dindo score of $\geq 3$ as a major complication. The result of PDS was categorised into complete (no macroscopic tumour residual), optimal (largest tumour residual $\leq 1 \mathrm{~cm}$ ) or incomplete (largest tumour residual $>1$ $\mathrm{cm})$. Patients were divided into two age groups with a threshold of 65 years. A body mass index $(\mathrm{BMI})>30$ was considered obese.

\section{Body composition analysis}

Axial CT at L3 was used for evaluation of total skeletal muscle (SM), psoas muscle, intramuscular adipose tissue (IMAT), visceral adipose tissue (VAT) and subcutaneous adipose tissue (SAT). Predefined and previously validated boundaries of -30 to +150 Hounsfield Units (HU) for SM and psoas, -190 to $-30 \mathrm{HU}$ for IMAT and SAT, and -150 to -50 HU for VAT were used to demarcate tissue using SliceOmatic software (v5.0, Tomovision, Montreal, Canada), see figure 1 . Surface areas in $\mathrm{cm}^{2}$ were quantified automatically following demarcation and were standardised by height squared to compute the skeletal muscle index (SMI), psoas-index, IMAT-index, VAT-index and SAT-index in $\mathrm{cm}^{2} / \mathrm{m}^{2}$. Mean HU was calculated for SM to define the muscle radiation attenuation (MA). CT evaluation was performed by two observers (IR\&JU) blinded to each other and to patient outcome. Their averaged measurements were used for statistical analysis. 


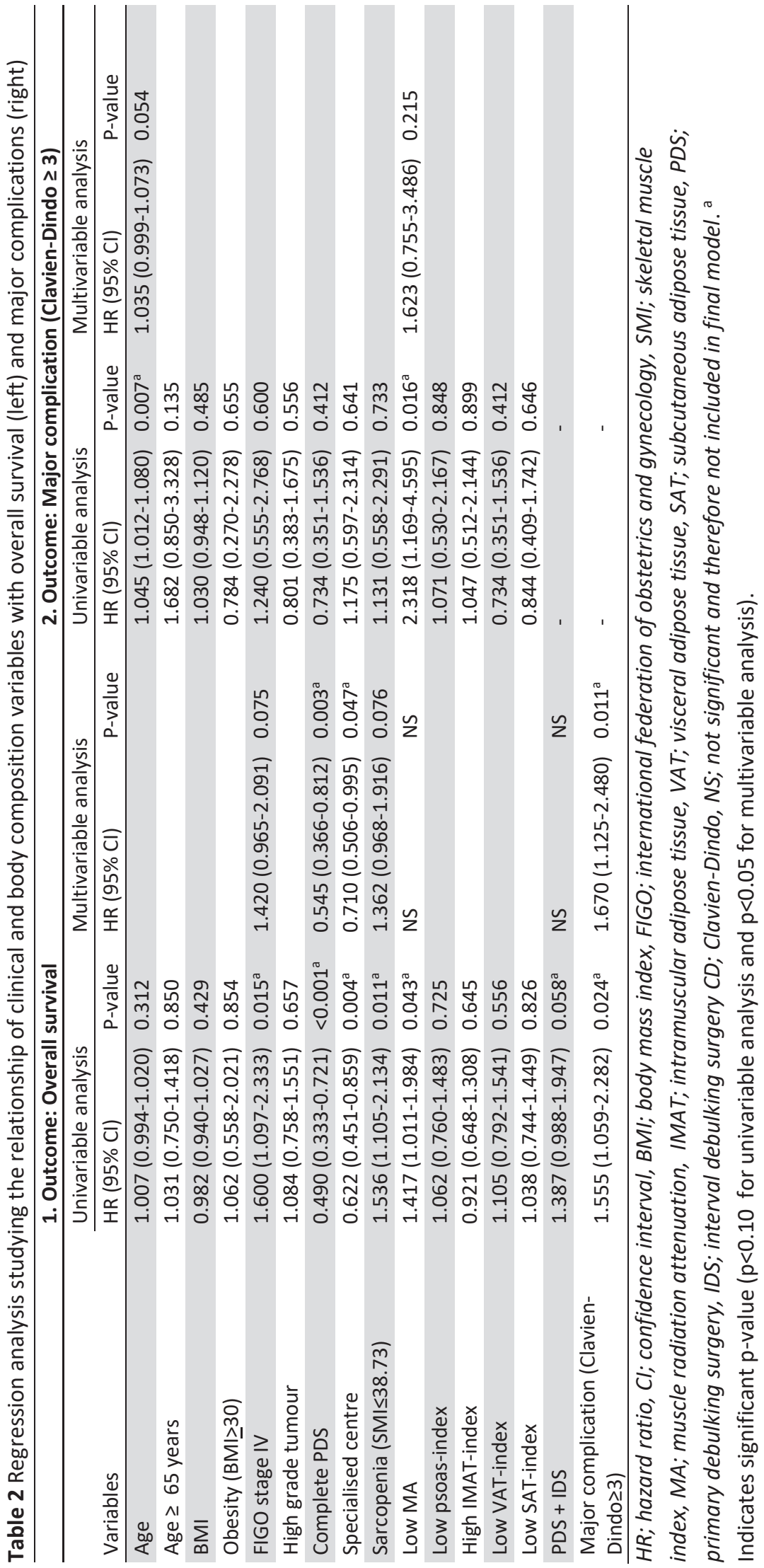




\section{Statistical analysis}

We performed optimum stratification analysis on the SMI measurements to stratify patients into two groups: patients with sarcopenia and patients without sarcopenia. This method produces a p-value for each SMI cut-off and determines the optimal cut-off for sarcopenia in our population [24]. Psoas-index, IMAT-index, VAT-index, SAT-index and MA were divided into tertiles and the lowest tertile was used as cut-off for each category (with an exception for IMAT-index in which the highest tertile was used) to divide patients into a 'low' or 'high' subgroup. Baseline data between patients with and without sarcopenia were analysed with chi-squared and t-tests. Differences in OS between sarcopenic and non-sarcopenic patients were tested with the Kaplan-Meier estimate (log rank). Body composition measurements as described above, FIGO stage (<IV vs. IV), age, BMI, outcome of PDS (complete vs. optimal/incomplete), tumour grade ( 1 vs. $2 / 3$ ), treatment centre (specialised vs. general), type of treatment (PDS vs. PDS+IDS) and development of major complications (Clavien-Dindo $\geq 3$ ) were tested as effect modifiers in regression analysis adopting a backwards stepwise procedure. A proportional hazards Cox-regression model was used to evaluate relationships with OS and a binary logistic regression model was used to study predictors for the development of major complications at PDS. Hazard ratios (HR) were calculated with confidence intervals $(95 \% \mathrm{Cl})$. For univariable analysis an inclusion criterion of $10 \%$ was adopted. Significant modifiers were included in a multivariable model in a backwards stepwise procedure in which a $\mathrm{p}$-value $<0.05$ was considered significant. For all statistical analyses SPSS v23.0 (IBM Corp, Chicago, IL) was used. Interobserver agreement for measurement of body composition variables was calculated with Pearson's correlation coefficient $\left(r_{p}\right)$. Pearson's $r$ was also used to evaluate the correlation between SMI and psoas-index.

\section{Results}

Of 280 patients deemed eligible to participate, 64 were excluded. 216 patients were included in the analyses (Figure 2). Patient characteristics are summarised in Table 1. Mean period between CT and PDS was 21 days. Mean OS was 1714 days. Forty-three patients (19.9\%) experienced a major postoperative complication. A more elaborate list of complications can be found in the supplementary material. Sarcopenic patients had a significantly lower mean BMI compared to non-sarcopenic patients ( $\left.22.7 \mathrm{vs.} 26.0 \mathrm{~kg} / \mathrm{m}^{2}, \mathrm{p}<0.001\right)$. Ascites was more prevalent in sarcopenic patients $(87.1 \%$ versus $65.7 \%, \mathrm{p}=0.010)$. Furthermore SMI, psoasindex, VAT-index and SAT-index were all significantly lower in patients with sarcopenia (all $\mathrm{p}<0.001)$. The outcome of PDS was not significantly different between sarcopenic and nonsarcopenic patients $(25.7 \%$ vs. $35.6 \%$ complete, $p=0.346)$.

Optimum stratification analysis identified an $\mathrm{SMI} \leq 38.73 \mathrm{~cm}^{2} / \mathrm{m}^{2}$ as the most optimal cut-point for sarcopenia in our population. Using this cut-point, Kaplan-Meier analysis showed a significant survival disadvantage for patients with sarcopenia compared to patients without sarcopenia ( $p=0.010$, Figure 3). Sarcopenia univariably predicted OS (HR $1.536(95 \% \mathrm{Cl} 1.105-$ 2.134), $p=0.011$ ) but was not significant at the level of $5 \%$ in multivariable Cox-regression analysis (HR $1.362(95 \% \mathrm{Cl} 0.968-1.916) \mathrm{p}=0.076$, Table 2). Significant predictors for OS in multivariable Cox-regression analysis were complete PDS (HR $0.545(95 \% \mathrm{Cl} \quad 0.366-0.812)$, $\mathrm{p}=0.003)$, specialised centre $(\mathrm{HR} 0.710(95 \% \mathrm{Cl} 0.506-0.995) \mathrm{p}=0.047)$ and the development of major complications (HR $1.670(95 \% \mathrm{Cl} 1.125-2.480), \mathrm{p}=0.011) . \quad$ Sarcopenic patients did not develop more severe complications than non-sarcopenic patients (Table 1). 
Sarcopenia was also not predictive of a major complication in logistic regression analysis (Table 2). Age and low MA were univariably identified as predictors for major complications but were not significant in multivariable analysis (Table 2).

Interobserver agreement was 0.970, 0.989, 0.969, 0.997, 0.994 and 0.995 for SM, psoas, IMAT, VAT, SAT and MA respectively. Correlation between measurements of SMI and psoas-index was 0.453 .

Figure 1 Computed tomography assessment of skeletal muscle and adipose tissue with SliceOmatic software

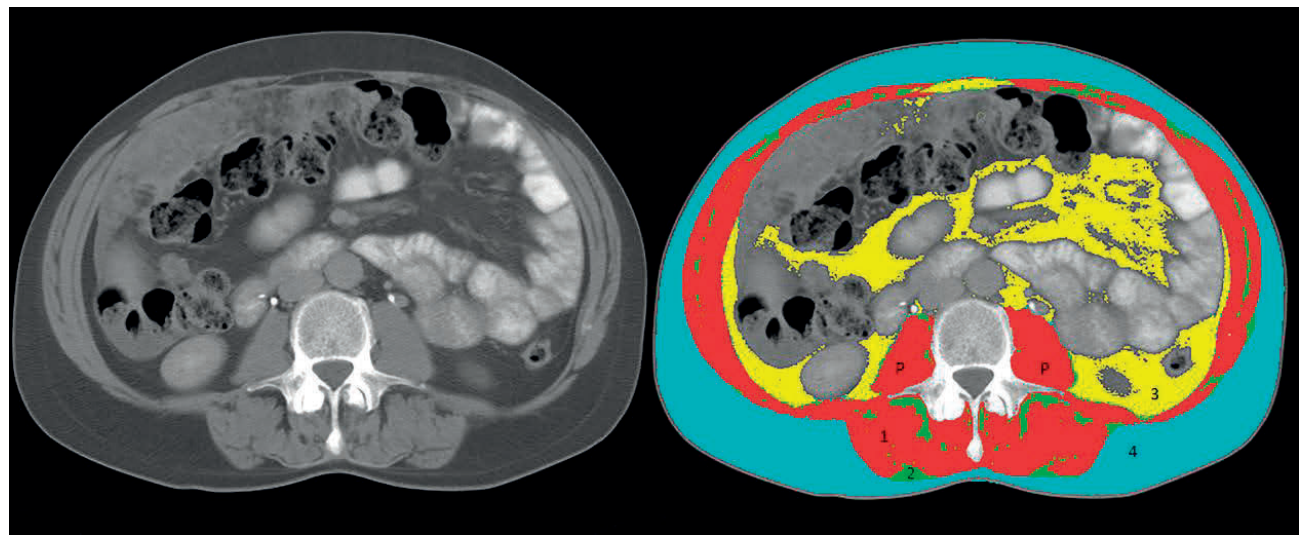

Preoperative axial computed tomography (CT) scan of an ovarian cancer patient. Both images are taken from the same patient. On the left a regular CT image at the level of the third lumbar vertebra. On the right an image coloured using SliceOmatic software. 1= skeletal muscle, $2=$ intramuscular adipose tissue, 3 = visceral adipose tissue, 4 = subcutaneous adipose tissue, $\mathrm{P}=$ psoas muscle.

Figure 2 Flow chart for patient inclusion

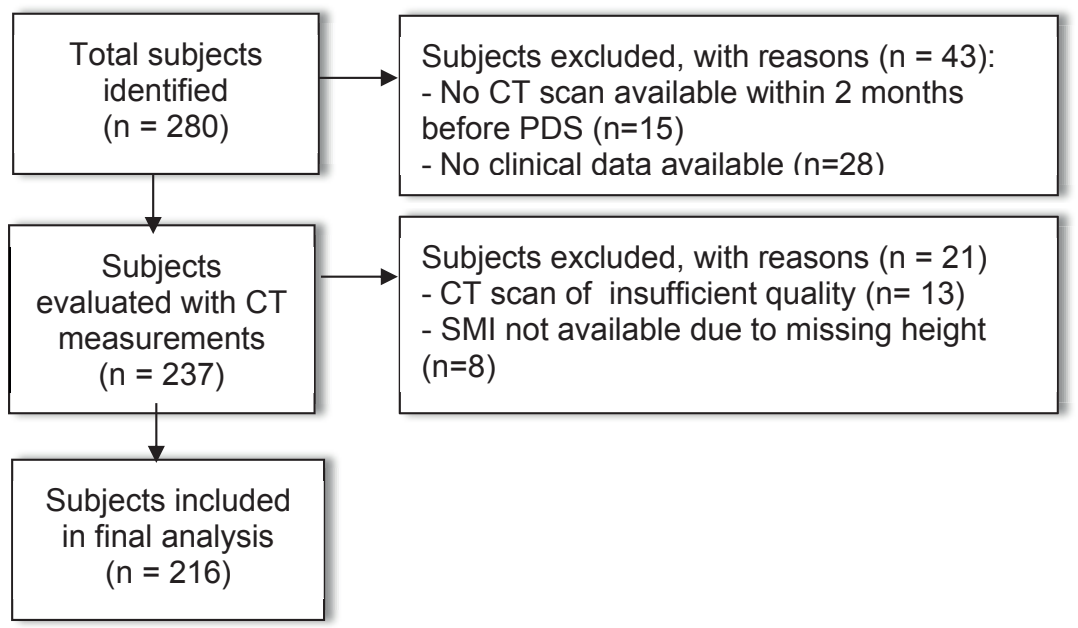

CT; computed tomography, PDS; primary debulking surgery, SMI; skeletal muscle index 


\section{Discussion}

In this study we evaluated the role of skeletal muscle measurements in prediction of OS and the development of post-surgical complications in ovarian cancer patients treated with PDS. We found a strong trend towards a survival disadvantage for patients with sarcopenia but the relationship was not significant in multivariable regression analysis. Tumour stage, completeness of PDS, treatment in a specialised centre and the development of severe postsurgical complications were stronger predictors for ovarian cancer survival. Secondly we did not establish a relationship between sarcopenia and the development of complications after PDS.

A recent meta-analysis concluded that low SMI was associated with poor OS among various tumour types [13]. Unfortunately no gynaecological malignancies were included in the meta-analysis and individual studies on ovarian cancer have not been able to confirm this association. Two retrospective studies about ovarian cancer patients undergoing PDS found no relationship between low SM and survival $[15 ; 16]$. Further, in a cohort of ovarian cancer patients undergoing neoadjuvant chemotherapy and IDS an initial low SMI measurement before treatment had no prognostic relevance but a change in muscle during chemotherapy was highly predictive of OS [14]. The present study shows no significant relationship between sarcopenia and OS when corrected for other prognostic variables but does imply a tendency towards a shorter survival nonetheless as is seen in Kaplan-Meier analysis and univariable regression. It is possible that the effect of sarcopenia was substantially diminished due to the influence of other strong prognostic predictors such as the outcome of debulking surgery. Furthermore, the impact of sarcopenia on OS might become more apparent when studying a larger population, which may also explain why previously published (relatively small) studies were not able to show a connection between sarcopenia and survival.

We encountered some obstacles in this study when we were faced with the choice of a cut-point for sarcopenia. SMI cut-points of $29.6-42.1 \mathrm{~cm}^{2} / \mathrm{m}^{2}$ have been reported for female cancer patients [13]. Although the majority of studies have used a cut-point between 38.5$41.0 \mathrm{~cm}^{2} / \mathrm{m}^{2}$ for females, this has still resulted in a very heterogeneous reported incidence of $15-74 \%$ patients classifying as sarcopenic [13]. Cancer type and stage, interpersonal variation of muscle mass, obesity and ethnicity can all influence SMI and to define a single 'gold standard' cut-point for sarcopenia is virtually impossible $[25 ; 26]$. We revised previous oncologic studies but cut-points for gynaecological cancer patients specifically were nonexistent. In our opinion cut-points designed for and applied to gastrointestinal cancer patients were less applicable to our population since these cancers have a stronger relationship with nutrition and metabolism in general. Although gynaecological tumours and urological tumours have differences in presentation, pathology and prognosis, they both do not affect nutrient uptake and metabolism directly Due to a lack of other suitable comparable cancers we investigated cut-points used in populations with urological cancers. Psutka et al. studied patients with renal cell cancer and urothelial cancer and found a relationship between sarcopenia and survival when using an SMI cut-point of $39 \mathrm{~cm}^{2} / \mathrm{m}^{2}$ [11; 27]. The cut-point of $39 \mathrm{~cm}^{2} / \mathrm{m}^{2}$ in these studies was chosen in accordance with guidelines defined by an international consensus group [6]. However, this guideline dates back to 2011 and was strongly based on the outcome of a study investigating respiratory and gastrointestinal tumours in an obese population [28]. Due to the absence of a suitable existing cut-point for ovarian cancer patients we used optimum stratification to determine the optimal cut-point for sarcopenia in our own cohort. To investigate if a different sarcopenia cut-point would 
influence the relationship with OS we performed a post-hoc analysis using two alternative cutpoints: 1) $39 \mathrm{~cm}^{2} / \mathrm{m}^{2}$ from the existing literature on urological tumours and 2) the lowest tertile SMI from our own population which was $38.87 \mathrm{~cm}^{2} / \mathrm{m}^{2}$. These cut-points were very close to the cut-point of $38.73 \mathrm{~cm}^{2} / \mathrm{m}^{2}$ used in this study and expectedly did not have a substantial impact on outcome. Both alternative cut-points resulted in a significant univariable but not multivariable relationship between sarcopenia and OS although they also showed a strong tendency towards a relation. In fact, all cut-points between 37.90 and 40.25 showed univariable significant relationships with OS for our population.

Interobserver agreement between the two observers was very strong ( $r_{p} 0.969$ - 0.997). Quantification of skeletal muscle and adipose tissue with SliceOmatic software is highly reproducible. Although the mean VAT- and SAT-index were significantly lower in patients with sarcopenia compared to patients without sarcopenia, neither measurements of adipose tissue were predictive of OS or complications. The psoas-index weakly correlated with the SMI and was not predictive of survival or complications thus we strongly advise against using it as substitute for the SMI. Aust et al. previously identified MA as a prognostic factor for OS in ovarian cancer patients [16]. We could not establish this relationship in our population.

From our results we can conclude that sarcopenia does not predict the development of major complications after PDS for ovarian cancer. This is in agreement with the only other ovarian cancer study that evaluated sarcopenia in relation to surgical complications [15]. However researchers in other cancer types have been able to connect sarcopenia to the risk of surgical complications although the results are inconsistent. We hypothesize that this difference may be (partially) explained by the aggressiveness of the surgical procedure. The extensiveness of surgery is possibly more limited in ovarian cancer patients than for instance in patients undergoing resections for gastro-intestinal malignancies. When it is apparent that a patient with ovarian cancer will have to undergo extensive surgery including splenectomy, (partial) hepatectomy, and/or multiple resections of large or small bowel, patients are often primarily treated with neoadjuvant chemotherapy [3; 29]. In this way the risk of complications during a possible interval debulking is decreased with an expected decrease of tumour burden. Patients undergoing more extensive surgery are also more likely to develop severe complications which could explain why a relationship between sarcopenia and complications might be more prominent in other cancer types because the complication rate is higher.

Due to the retrospective nature of our study we encountered a substantial amount of irretrievable data which explains why 64 patients from the original cohort were excluded. We would also have liked to analyse the influence of performance status on sarcopenia and survival but this information was unfortunately missing in over $50 \%$ of patients.

\section{Conclusion}

Sarcopenia was not predictive of overall survival or major surgical complications in ovarian cancer patients undergoing primary debulking surgery. Other prognostic factors were stronger predictors for survival. However we did see a strong trend towards a survival disadvantage for patients with sarcopenia. Previous investigations have shown that loss of skeletal muscle during chemotherapy was related to decreased survival in ovarian cancer and the measurement of muscle change might be more important than a single measurement before treatment [14]. Sarcopenia seems to play a noticeable role in ovarian cancer. Whether sarcopenia can be modified with therapy is still unknown. Nutritional and exercise interventions have been found to improve muscle function in sarcopenic elderly [19]. 
However, readily implementable anti-sarcopenia protocols for cancer patients are unfortunately not yet available. Future prospective studies should focus on investigation of the metabolic phenotype of patients with sarcopenia and to assessing whether interventions (e.g. nutritional support, anti-inflammatory medication and physical exercise) have an effect in cancer patients. For the time being it remains pivotal however to achieve complete cytoreduction during ovarian cancer debulking surgery as this has shown to be the strongest predictor of overall survival.

Figure 3 Kaplan-Meier survival analysis

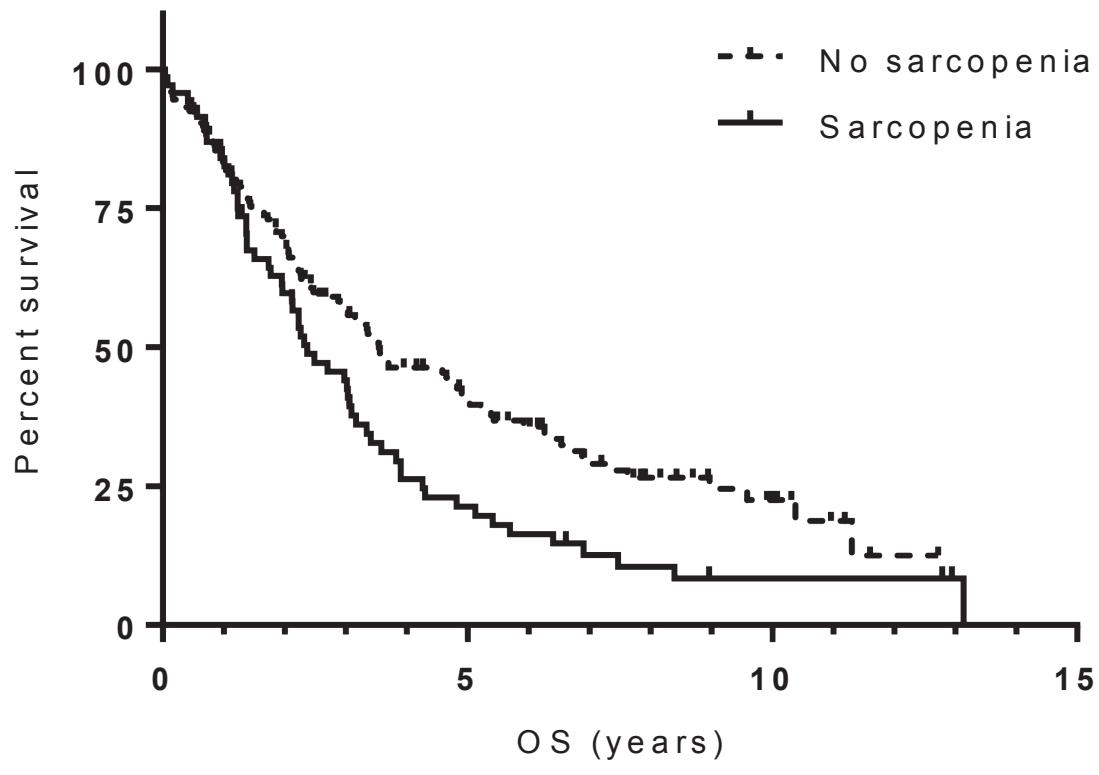

Number of subjects at risk (\%)

\begin{tabular}{llllll} 
& $0 \mathrm{yr}$ & $1 \mathrm{yr}$ & $2 \mathrm{yr}$ & $5 \mathrm{yr}$ & $10 \mathrm{yr}$ \\
\hline No Sarcopenia & $146(100)$ & $117(80)$ & $90(62)$ & $44(30)$ & $10(7)$ \\
Sarcopenia & $70(100)$ & $57(81)$ & $40(57)$ & $14(20)$ & $4(6)$ \\
\hline
\end{tabular}

OS; overall survival. Log rank estimate: $\mathrm{p}=0.010$. 


\section{References}

1. Torre LA, Bray F, Siegel RL, Ferlay J, Lortet-Tieulent J, Jemal A (2015) Global cancer statistics, 2012. CA Cancer J Clin 65:87-108

2. Baldwin LA, Huang B, Miller RW et al (2012) Ten-year relative survival for epithelial ovarian cancer. Obstet Gynecol 120:612-618

3. Vergote I, Trope CG, Amant F et al (2010) Neoadjuvant chemotherapy or primary surgery in stage IIIC or IV ovarian cancer. N Engl J Med 363:943-953

4. du Bois A, Reuss A, Pujade-Lauraine E, Harter P, Ray-Coquard I, Pfisterer J (2009) Role of surgical outcome as prognostic factor in advanced epithelial ovarian cancer: a combined exploratory analysis of 3 prospectively randomized phase 3 multicenter trials: by the Arbeitsgemeinschaft Gynaekologische Onkologie Studiengruppe Ovarialkarzinom (AGOOVAR) and the Groupe d'Investigateurs Nationaux Pour les Etudes des Cancers de I'Ovaire (GINECO). Cancer 115:1234-1244

5. Tisdale MJ (2002) Cachexia in cancer patients. Nat Rev Cancer 2:862-871

6. Fearon K, Strasser F, Anker SD et al (2011) Definition and classification of cancer cachexia: an international consensus. Lancet Oncol 12:489-495

7. Shen W, Punyanitya M, Wang Z et al (2004) Visceral adipose tissue: relations between singleslice areas and total volume. Am J Clin Nutr 80:271-278

8. Shen W, Punyanitya M, Wang Z et al (2004) Total body skeletal muscle and adipose tissue volumes: estimation from a single abdominal cross-sectional image. J Appl Physiol (1985) 97:2333-2338

9. Mourtzakis M, Prado CM, Lieffers JR, Reiman T, McCargar LJ, Baracos VE (2008) A practical and precise approach to quantification of body composition in cancer patients using computed tomography images acquired during routine care. Appl Physiol Nutr Metab 33:997-1006

10. van Vledder MG, Levolger S, Ayez N, Verhoef C, Tran TC, Ijzermans JN (2012) Body composition and outcome in patients undergoing resection of colorectal liver metastases. Br J Surg 99:550557

11. Psutka SP, Boorjian SA, Moynagh MR et al (2016) Decreased Skeletal Muscle Mass is Associated with an Increased Risk of Mortality after Radical Nephrectomy for Localized Renal Cell Cancer. J Urol 195:270-276

12. Martin L, Birdsell L, Macdonald N et al (2013) Cancer cachexia in the age of obesity: skeletal muscle depletion is a powerful prognostic factor, independent of body mass index. J Clin Oncol 31:1539-1547

13. Shachar SS, Williams GR, Muss HB, Nishijima TF (2016) Prognostic value of sarcopenia in adults with solid tumours: A meta-analysis and systematic review. Eur J Cancer 57:58-67

14. Rutten IJG, Van Dijk DPJ, Kruitwagen RFPM, Beets-Tan RGH, Olde Damink SW, Van Gorp T (2016) Loss of skeletal muscle during neoadjuvant chemotherapy is related to decreased survival in ovarian cancer patients. Journal of Cachexia, Sarcopenia and Muscle. 10.1002/jcsm.12107

15. Torres ML, Hartmann LC, Cliby WA et al (2013) Nutritional status, CT body composition measures and survival in ovarian cancer. Gynecol Oncol 129:548-553

16. Aust S, Knogler T, Pils D et al (2015) Skeletal Muscle Depletion and Markers for Cancer Cachexia Are Strong Prognostic Factors in Epithelial Ovarian Cancer. PLoS One 10:e0140403

17. Lieffers JR, Bathe OF, Fassbender K, Winget M, Baracos VE (2012) Sarcopenia is associated with postoperative infection and delayed recovery from colorectal cancer resection surgery. $\mathrm{Br} J$ Cancer 107:931-936 
18. Fukuda Y, Yamamoto K, Hirao M et al (2015) Sarcopenia is associated with severe postoperative complications in elderly gastric cancer patients undergoing gastrectomy. Gastric Cancer. 10.1007/s10120-015-0546-4

19. Rondanelli M, Klersy C, Terracol G et al (2016) Whey protein, amino acids, and vitamin D supplementation with physical activity increases fat-free mass and strength, functionality, and quality of life and decreases inflammation in sarcopenic elderly. Am J Clin Nutr 103:830-840

20. Gould DW, Lahart I, Carmichael AR, Koutedakis Y, Metsios GS (2013) Cancer cachexia prevention via physical exercise: molecular mechanisms. J Cachexia Sarcopenia Muscle 4:111124

21. Maddocks M, Murton AJ, Wilcock A (2012) Therapeutic exercise in cancer cachexia. Crit Rev Oncog 17:285-292

22. Bowen TS, Schuler G, Adams V (2015) Skeletal muscle wasting in cachexia and sarcopenia: molecular pathophysiology and impact of exercise training. J Cachexia Sarcopenia Muscle 6:197-207

23. Dindo D, Demartines N, Clavien PA (2004) Classification of surgical complications: a new proposal with evaluation in a cohort of 6336 patients and results of a survey. Ann Surg 240:205-213

24. Williams BA, N. Mandrekar JN, Mandrekar SJ, Cha SS, Furth AF (2006) Finding Optimal Cutpoints for Continuous Covariates with Binary and Time-to-Event Outcomes. Technical Report Series \#79. Mayo Foundation

25. Jones A, Jr., Shen W, St-Onge MP et al (2004) Body-composition differences between African American and white women: relation to resting energy requirements. Am J Clin Nutr 79:780786

26. Tan L, Liu SL, Lei SF, Papasian CJ, Deng HW (2012) Molecular genetic studies of gene identification for sarcopenia. Hum Genet 131:1-31

27. Psutka SP, Carrasco A, Schmit GD et al (2014) Sarcopenia in patients with bladder cancer undergoing radical cystectomy: impact on cancer-specific and all-cause mortality. Cancer 120:2910-2918

28. Prado CM, Lieffers JR, McCargar $L$ et al (2008) Prevalence and clinical implications of sarcopenic obesity in patients with solid tumours of the respiratory and gastrointestinal tracts: a population-based study. Lancet Oncol 9:629-635

29. Vergote I, du Bois A, Amant F, Heitz F, Leunen K, Harter P (2013) Neoadjuvant chemotherapy in advanced ovarian cancer: On what do we agree and disagree? Gynecol Oncol 128:6-11 
5 


\section{No influence of sarcopenia on survival of ovarian cancer patients in a prospective validation study}

Gynecologic Oncology (2020)

Jorne Ubachs, Simone Koole, Max Lahaye, Cristina Fabris, Leigh Bruijs, Jules Schagen van Leeuwen, Henk Schreuder, Ralph Hermans, Ignace de Hingh, Jacobus van der Velden, Henriëtte Arts, Maaike van Ham, Peter van Dam, Patrick Vuylsteke, Jacco Bastings, Roy Kruitwagen, Sandrina Lambrechts, Steven Olde Damink, Sander Rensen, Toon Van Gorp, Gabe Sonke, Willemien van Driel 


\begin{abstract}
Objective

Decreases in skeletal muscle index (SMI) during neo-adjuvant chemotherapy (NACT) have been associated with worse outcome in patients with advanced ovarian cancer. To validate these findings, we tested if a decrease in SMI was a prognostic factor for a homogenous cohort of patients who received NACT in the randomized phase 3 OVHIPEC-trial.
\end{abstract}

\title{
Methods
}

CT-scans were performed at baseline and after two cycles of neo-adjuvant chemotherapy in stage III ovarian cancer patients. The SMI (skeletal muscle area in $\mathrm{cm}^{2}$ divided by body surface area in $\mathrm{m}^{2}$ ) was calculated using SliceOMatic software. The difference in SMI between both $\mathrm{CT}$-scans $(\triangle \mathrm{SMI})$ was calculated. Cox-regression analyses were performed to analyze the independent effect of a difference in $\mathrm{SMI}(\triangle \mathrm{SMI})$ on outcome. Log-rank tests were performed to plot recurrence-free (RFS) and overall survival (OS). The mean number of adverse events per patient were compared between groups using t-tests.

\section{Results}

Paired CT-scans were available for 212 out of 245 patients (87\%). Thirty-four of 74 patients (58\%) in the group with a decrease in $\triangle \mathrm{SMI}$ and 73 of 138 of the patients (53\%) in the group with stable/increase in $\triangle S M I$ had died. Median RFS and OS did not differ significantly $(p=0.297$ and $p=0.764$ ) between groups. Patients with a decrease in SMI experienced more preoperative adverse events, and more grade 3-4 adverse events.

\section{Conclusion}

Decreased SMI during neo-adjuvant chemotherapy was not associated with worse outcome in patients with stage III ovarian cancer included in the OVHIPEC-trial. However, a strong association between decreasing SMI and adverse events was found. 


\section{Introduction}

Epithelial ovarian cancer shows the highest mortality rate of all gynaecologic cancers in the western world[1]. The majority of patients is diagnosed with advanced stage disease, (FIGO $\geq \mathrm{III}$ [2]. For advanced stage patients, 10 -year survival is only $10-15 \%$, and long term survival has not improved over the past 20 years[3]. Standard treatment consists of maximum cytoreductive surgery (CRS) combined with chemotherapy consisting of carboplatin and paclitaxel. If complete primary CRS is not feasible, interval CRS might be performed after three cycles of neo-adjuvant chemotherapy[4-6]. Achieving a complete cytoreduction is the most important prognostic factor, in addition to the ability to complete six cycles of carboplatin and paclitaxel and the clinical condition of the patient $[7,8]$. The clinical condition is reflected by multiple factors and besides subjective scores, such as the World Health Organization (WHO) performance score, we lack an objective measure of patient fitness. Sarcopenia might be such a measure, and might be predictive for treatment completion and outcome in patients with advanced stage ovarian cancer.

Sarcopenia is the loss of, or low, skeletal muscle mass[9]. The skeletal muscle mass of patients with cancer can be accurately quantified using a single lumbar CT image by the so-called skeletal muscle index (SMI)[10]. Loss of skeletal muscle mass is associated with higher rates of chemotoxicity and impaired overall survival (OS) in ovarian cancer patients[11-13]. In an analysis of a retrospective cohort of 123 patients published by Rutten et al., loss of skeletal muscle mass during NACT was shown to be associated with worse outcome. However, multivariate analyses taking into account completeness of surgery, response to chemotherapy, and toxicity were not performed[13]. Although previous studies reported worse outcome in patients with ovarian cancer and either a low baseline SMI or a decrease in $\mathrm{SMI}$ during treatment, it is uncertain whether SMI is an independent prognostic factor or a measure of extensive disease and treatment burden and poor performance[14-16].

The aim of this study was to validate earlier results by Rutten et al. in the most homogenous cohort of ovarian cancer patients reported to date. We analyzed whether a decrease in SMI during NACT was associated with worse outcome in a cohort of patients with similar extent of disease; stage III epithelial ovarian cancer, and similar performance scores. 


\section{Patients and methods}

\section{Patients and treatment}

For this study, CT-scans were collected from patients included in the OVHIPEC trial[17]. This multicenter randomized phase III trial included 245 newly-diagnosed patients with stage III epithelial ovarian, fallopian tube, or peritoneal cancer between April 2007 and April 2016. Full eligibility criteria are presented elsewhere[17]. All patients received three cycles of intravenous chemotherapy (carboplatin [area under the curve $5-6 \mathrm{mg} / \mathrm{ml}$ per minute] and paclitaxel $\left[175 \mathrm{mg} / \mathrm{m}^{2}\right]$ ) prior to interval cytoreductive surgery, because of extent of disease, or because of incomplete primary CRS (residual tumor $>1 \mathrm{~cm}$ in diameter). Patients were eligible for inclusion in case of at least stable disease after two cycles of NACT. Randomization was performed during interval cytoreductive surgery when complete or optimal (no visible, or $<1 \mathrm{~cm}$ visible tumor remaining) CRS was anticipated. Patients were randomized to receive either interval CRS with or without HIPEC using cisplatin $100 \mathrm{mg} / \mathrm{m}^{2}$. In both groups disease was too extensive for primary surgery, either defined by incomplete primary surgery or based on radiological imaging. Ten percent of patients included in the OVHIPEC-trail were primarily treated with incomplete primary cytoreduction before administration of chemotherapy and complete or near-complete interval cytoreduction, numbers were equally distributed between arms due to randomization. Subgroup analysis in the OVHIPEC study showed no difference in outcome between patients treated with initial incomplete surgery and those who started with neo-adjuvant chemotherapy. An additional three cycles of adjuvant intravenous carboplatin were administered after CRS for all patients. CT-scans were performed at diagnosis: before start of neo-adjuvant chemotherapy (NACT), after 2 cycles of NACT, at the end of adjuvant chemotherapy, and at 6, 12, and 24 months after the last cycle of chemotherapy. Grade 1-5 toxicity was scored from baseline to 30 days after end of therapy, using the National Cancer Institute Common Terminology Criteria for Adverse Events (CTCAE) version 4.0. All patients provided informed consent for participation. The trial protocol was approved by the institutional review board of the Netherlands Cancer Institute. The trial was registered in the international database: ClinicalTrials.gov Identifier: NCT00426257.

\section{Body composition analysis}

To analyze whether pre-operative sarcopenia and/or a skeletal muscle depletion were associated with worse outcome, CT-scans performed at baseline (timepoint 1 ) and after 2 cycles of neo-adjuvant chemotherapy (timepoint 2 ) were selected. The axial slide at the third lumbar level (L3), with the best representation of the bilateral transverse process was selected from each portal venous phase CT-scan. The axial CT-scan at the L3 level was used for evaluation of total skeletal muscle (SM). Predefined and previously validated cut-offs of -29 to +150 Hounsfield Units (HU)[18] for SM were used to demarcate tissue using SliceOmatic software (v5.0, Tomovision, Montreal, Canada). Evaluation of the demarcations was performed by one of two observers (JU \& JB), both blinded for clinical characteristics, outcome and allocated treatment arm. An example of how tissues were delineated with SliceOmatic is shown in Figure 1. The Skeletal Muscle Index (SMI) was calculated using the surface area of $\mathrm{SM}$ in $\mathrm{cm}^{2}$ and the stature of the patient (squared height) in $\mathrm{m}^{2}$. All data were coded and processed anonymously.

Changes in SMI from timepoint 1 to timepoint 2 were expressed as a percentage. To account for variations in time between the sequential CT-scans, the change in SMI was calculated as a 
percentage of change per 100 days. In order to do so, the change in SMI between the scan at timepoint 1 and timepoint 2 was divided by the number of days between the scans, and subsequently multiplied by 100 . This is referred to as $\Delta \mathrm{SMI}$. A measurement error of $2 \%$ was adopted based on previously reported accuracy of CT for muscle and fat tissue analysis. [10] Patients with a decrease of $>2 \%$ of SMI in 100 days were defined as the SMI-loss group. The $2 \%$ cutoff was also used by Rutten et al.[13]. Other patients were defined as SMI-stable or increase[10, 13].

Only CT-scans that were performed up to 10 weeks before start of chemotherapy, up to interval CRS surgery were selected. Scans at timepoint 1 had to be performed ten weeks to 1 day before start of neo-adjuvant chemotherapy. Scans at timepoint 2 had to be performed more than three weeks after start of neo-adjuvant chemotherapy but before interval CRS.

Figure 1 Abdominal axial CT scan of an ovarian cancer patient taken at the third lumbar level. Total skeletal muscle area in red.

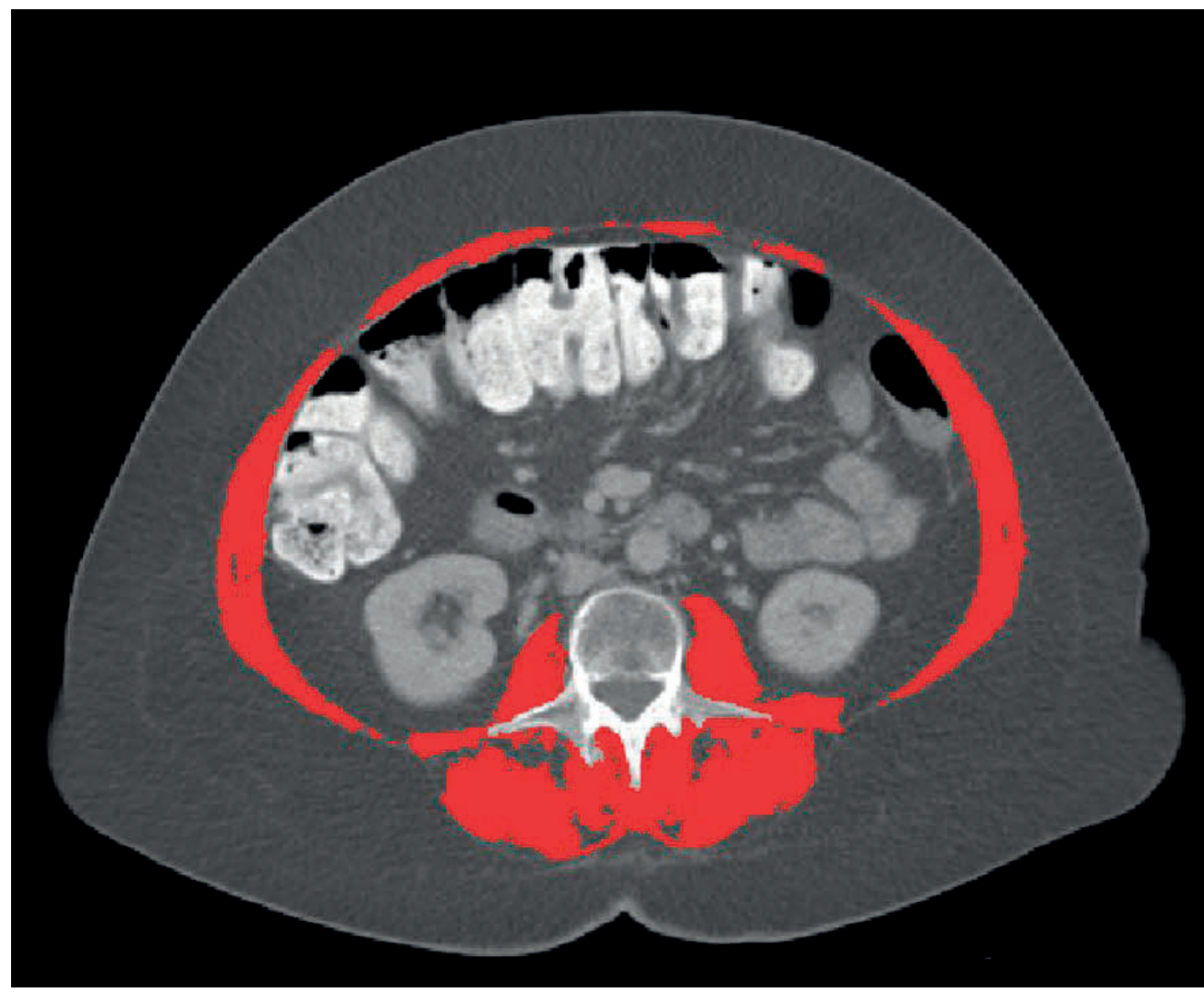


Table 1 Patient and treatment characteristics

\begin{tabular}{|c|c|c|c|c|}
\hline & $\begin{array}{l}\text { Total cohort } \\
\qquad \mathrm{N}=212\end{array}$ & $\begin{array}{c}\text { SMI decrease } \\
>2 \% \\
N=138\end{array}$ & $\begin{array}{c}\text { SMI stable or SMI } \\
\text { increase } \\
\mathrm{N}=74\end{array}$ & $\begin{array}{l}\text { P- } \\
\text { value }\end{array}$ \\
\hline $\begin{array}{l}\text { Age, mean years } \\
\text { (SD) }\end{array}$ & $60.9(8.1)$ & $60.9(8.2)$ & $61.0(7.8)$ & 0.883 \\
\hline \multicolumn{5}{|l|}{ FIGO, Nr. (\%) } \\
\hline III & $221(100)$ & $138(100)$ & $74(100)$ & \\
\hline \multicolumn{4}{|l|}{$\begin{array}{l}\text { Histological type } \\
\text { (\%) }\end{array}$} & 0.469 \\
\hline High-grade serous & $191(90.1)$ & $125(90.6)$ & $66(89.2)$ & \\
\hline Other & $19(9.0)$ & $11(8.0)$ & $8(10.8)$ & \\
\hline Unknown & $2(0.9)$ & $2(1.4)$ & 0 & \\
\hline \multicolumn{4}{|c|}{ BMI, mean kg/m² (SD)* } & 0.025 \\
\hline Baseline & $20.5(3.5)$ & $20.9(3.6)$ & $19.7(3.1)$ & \\
\hline \multicolumn{4}{|c|}{ Weight, mean kg (SD)* } & 0.076 \\
\hline Baseline & $68.9(12.9)$ & $70.1(13.2)$ & $66.8(12.2)$ & \\
\hline \multicolumn{4}{|c|}{ Primary incomplete debulking, Nr. (\%) } & 0.884 \\
\hline yes & $18(8.5)$ & $12(8.7)$ & $6(8.1)$ & \\
\hline no & $194(91.5)$ & $126(91.3)$ & $68(91.9)$ & \\
\hline \multicolumn{4}{|c|}{ Outcome interval CRS, Nr. (\%)† } & 0.936 \\
\hline $\mathrm{R} 1$ & $146(68.9)$ & $95(68.8)$ & $51(68.9)$ & \\
\hline $\mathrm{R} 2 \mathrm{a}$ & $40(18.9)$ & $26(18.8)$ & $14(18.9)$ & \\
\hline $\mathrm{R} 2 \mathrm{~b}$ & $22(10.4)$ & $14(10.1)$ & $8(10.8)$ & \\
\hline Suboptimal & $1(0.5)$ & $1(0.7)$ & 0 & \\
\hline No resection & $1(0.5)$ & $1(0.7)$ & 0 & \\
\hline No surgery & $2(0.9)$ & $1(0.7)$ & $1(1.4)$ & \\
\hline \multicolumn{4}{|c|}{ Treatment with HIPEC, Nr (\%) } & 0.045 \\
\hline HIPEC & $103(48.6)$ & $74(53.6)$ & $29(39.2)$ & \\
\hline No HIPEC & $109(51.4)$ & $64(46.4)$ & $45(60.8)$ & \\
\hline \multicolumn{4}{|c|}{ Six cycles of chemotherapy completed, Nr. (\%) } & 0.895 \\
\hline Yes & $197(92.9)$ & $128(92.8)$ & $69(93.2)$ & \\
\hline No & $15(7.1)$ & $10(7.2)$ & $5(6.8)$ & \\
\hline
\end{tabular}

Table 1 CT; computed tomography, SMI; skeletal muscle index, N; number, FIGO; International Federation of Gynecology and Obstetrics, BMI; body mass index, CRS; cytoreductive surgery, NA; not applicable, HIPEC; hyperthermic intraperitoneal chemotherapy, $\mathrm{Nr}$; number

* based on known values

† R1: no visible tumor, complete cytoreduction; R2a: tumor nodules $\leq 2.5 \mathrm{~mm}$; R2b: tumor nodules $>2.5 \mathrm{~mm}$ and $\leq 10 \mathrm{~mm}$; suboptimal: tumor nodules $>10 \mathrm{~mm}$, incomplete cytoreduction 


\section{Statistical analyses}

Comparisons between the SMI-loss and SMI-stable/increase group were performed with the t-test for continuous variables and the Pearson's chi-square test for categorical variables. Preoperative toxicity was scored before start of chemotherapy, during NACT, or between NACT and surgery. Toxicity was divided into CTCAE grade 1-2 or grade 3-5 toxicity. The mean number of events per patient were presented and compared between groups using a t-test for independent samples. The Welch t-test was used to compare the total number of preoperative adverse events.

Survival was compared for sarcopenic patients at timepoint 1 and timepoint 2, and for the $\mathrm{SMI}$-loss group and the SMI-stable or increase group. Analyses of overall survival (OS) and recurrence-free survival (RFS) were performed using Kaplan-Meier estimates and log-rank tests. RFS was defined as the time from randomization to first-recurrence or death, whichever occurred first, as was defined by the GCIG criteria.[19] Univariate and multivariable cox regression analyses was performed for analyzing the effect of the different treatment arms on outcome, with deltaSMI and treatment arm (HIPEC or no HIPEC) as associated variables. Subgroup analyses were performed for interval CRS and interval CRS + HIPEC groups for OS and RFS. Statistical significance for all comparisons was determined at $p<0.05$. All analyses were performed with the statistical software package SPSS v.25.0 (IBM Corp, Chicago, IL, USA). 
Table 2 CT-scans and body composition characteristics

\begin{tabular}{|c|c|c|c|c|}
\hline & Total cohort & $\begin{array}{c}\text { SMI decrease } \\
>2 \% \\
N=138\end{array}$ & $\begin{array}{l}\text { SMI stable or } \\
\text { SMI increase } \\
\quad N=74\end{array}$ & $\begin{array}{c}\text { P- } \\
\text { value }\end{array}$ \\
\hline \multicolumn{5}{|c|}{ Nr. of days between scan 1-2, mean (SD) } \\
\hline & $60.3(15.7)$ & $60.1(16.0)$ & $60.6(15.3)$ & 0.841 \\
\hline \multicolumn{5}{|c|}{ Nr. of days between start NACT and scan 2 , mean (SD)* } \\
\hline & $36.8(8.0)$ & $36.66(7.5)$ & $37.1(9.0)$ & 0.727 \\
\hline \multicolumn{5}{|l|}{ SMI, mean $\mathrm{cm}^{2} / \mathrm{m}^{2}$ (SD) } \\
\hline Baseline & $39.5(5.4)$ & $40.7(5.3)$ & $37.4(4.0)$ & $<0.001$ \\
\hline After 2 cycles of NACT & $38.1(5.0)$ & $37.8(5.0)$ & $38.6(5.0)$ & 0.252 \\
\hline$\%$ of change (range) & $\begin{array}{r}-5.9 \% \\
(-31.6 \% \text { to } \\
46.9 \%)\end{array}$ & $\begin{array}{r}-12.3 \\
(-31.6 \% \text { to }- \\
2.3 \%)\end{array}$ & $\begin{array}{r}6.0 \% \\
(-2.0 \%-46.9 \%)\end{array}$ & $<0.001$ \\
\hline \multicolumn{5}{|l|}{ SMRA, mean HU (SD)* } \\
\hline Baseline & $36.0(7.8)$ & $35.7(7.6)$ & $36.5(8.2)$ & 0.464 \\
\hline After 2 cycles of NACT & $36.6(7.5)$ & $35.6(7.2)$ & $38.5(7.7)$ & 0.007 \\
\hline $\begin{array}{l}\text { OS, median months } \\
(95 \% \mathrm{Cl})\end{array}$ & $41(36.11-45.89)$ & $41(34.18-47.82)$ & $41(35.54-46.46)$ & 0.764 \\
\hline $\begin{array}{l}\text { RFS, median months } \\
(95 \% \mathrm{Cl})\end{array}$ & $12(10.63-13.37)$ & 11 (9.49-12.51) & $13(10.24-15.76)$ & 0.297 \\
\hline
\end{tabular}

Table 2 SMI; skeletal muscle index, nr; number, NACT; neo-adjuvant chemotherapy, SMRA; skeletal muscle radiation attenuation, HU; Hounsfield units, OS; overall survival, RFS; recurrence free survival, $95 \% \mathrm{Cl} ; 95 \%$ confidence interval

* based on known value 


\section{Results}

Paired CT-scans at baseline and after two cycles of NACT were available for 221 out of 245 patients $(90.2 \%)$ For 24 patients, CT-scans were not provided by the participating centers. Mean time between CT-scan 1 and CT-scan 2 was 60 days (SD 15.7). Mean time between cycles ofNACT and the interval CRS are presented in the supplementary material, table S1. The SMI and $\triangle \mathrm{SMI}$ could not be calculated for nine patients (4.1\%) because CT-scans were of insufficient quality or incomplete. The median SMI was 39.5 (range 27.5-57.9) at timepoint 1 and 38.1 (range 26.4-55.4) at timepoint 2. Means with SD of the SMI at timepoint 1 and 2, and $\Delta \mathrm{SMI}$ are presented in supplementary table S2. The $\Delta \mathrm{SMI}$ could be calculated for 212 patients. The mean $\Delta$ SMI was $-5.9 \%$ (SD $11.8 \%$ ), ranging from $-31.6 \%$ to $46.9 \% . \Delta S M I$ was lower than $2 \%$ in 138 patients and was stable or increased (higher than -2\%) in 74 patients. Baseline characteristics for all patients with SMI measurements are presented in Table 1 and 2. The mean BMI in the SMI stable or increase group was20.9 (SD 3.6) vs. 19.7 (SD 3.1) in the SMI decrease group and differed significantly $(p=0.025)$ between both groups. Slightly more patients with a decrease in SMI were treated with HIPEC ( $n=74(53.6 \%))$, compared to the group with stable/increased SMI ( $n=29(39.2 \%) p=0.045)$. Median OS was 41 months (95\% confidence interval [Cl] 36.1-45.9), and median RFS was 12 months (95\% Cl 10.6-13.4).

\section{Toxicity and sarcopenia}

Between enrollment and surgery, pre-operative toxicity was scored. A total of 1265 events were registered before interval cytoreductive surgery. The majority of the events was a CTCAE grade $1-2$ event $(1208 / 1265 ; 95.5 \%)$ (table 3$)$. Within the group of patients with a decrease in SMI, a total of 893 events (70.6\%) were reported, compared to $372(29.4 \%)$ events in the stable/increase SMI group ( $p=0.008)$. The overall mean number of pre-operative events per patient was 6.2 (SD 3.9). The percentage of grade 3-4 events was higher in the group with a decrease in SMI (5.3\%) than the percentage in the group with stable or increased SMI (2.6\%). The mean number of pre-operative events was significantly higher: 6.7 (SD 3.9) in the group of patients whose SMI decreased versus 5.3 (SD 3.1) in the group with a stable or increased SMI ( $p=0.019$ ) (Table 3). Specific grade 3-4 toxicities are listed in supplementary table S5, and were mostly related to chemotherapy or cancer-related adverse events such as pulmonary embolisms.

\section{Sarcopenia and survival}

After a median of 4.7 years of follow-up, 116 of 212 patients (55\%) had died and 181 of 212 patients (85\%) experienced an event of disease-recurrence or death. Survival analysis revealed that 43 of 74 patients (58\%) with a more than 2\% decrease in SMI versus 73 of $138(53 \%)$ of the patients with a stable or increased SMI had died. Median overall survival did not differ significantly between these two groups ( $p=0.764$, Figure 2). 61 of $74(82 \%)$ patients in the $\triangle \mathrm{SMI}$ decrease group had disease recurrence, compared to 120 of $138(87 \%)$ in the $\Delta \mathrm{SMI}$ stable/increase group. Median recurrence-free survival was similar ( $p=0.297$, Figure 3 ). $\Delta S M I$ was also not associated with overall survival and recurrence-free survival after correcting for treatment effect in multivariate analysis (supplementary tables S3 and S4, respectively). 
Table 3 association of decrease in SMI with adverse events

\begin{tabular}{|c|c|c|c|c|}
\hline & $\begin{array}{l}\text { Total cohort } \\
\qquad \mathbf{N}=\mathbf{2 0 3}\end{array}$ & $\begin{array}{c}\text { SMI decrease } \\
>2 \% \\
\mathrm{~N}=133\end{array}$ & $\begin{array}{l}\text { SMI stable or } \\
\text { SMI increase } \\
\quad N=70\end{array}$ & $\begin{array}{l}\text { P- } \\
\text { value }\end{array}$ \\
\hline \multicolumn{5}{|c|}{ Mean number of adverse events pre-operative (SD)* } \\
\hline Gr1-2 & $5.9(3.7)$ & $6.4(3.9)$ & $5.2(3.1)$ & 0.044 \\
\hline Gr3-4 & $0.3(0.7)$ & $0.4(0.8)$ & $0.1(0.4)$ & $<0.001$ \\
\hline $\begin{array}{l}\text { Mean } \mathrm{N} \text { of events pre-op per } \\
\text { pt }\end{array}$ & $6.2(3.9)$ & $6.7(4.2)$ & $5.3(3.1)$ & 0.019 \\
\hline \multicolumn{5}{|c|}{ Total number of adverse events pre-operative* } \\
\hline $\begin{array}{l}\text { Total Gr1-2 (range per } \\
\text { patient) }\end{array}$ & $1208(0-22)$ & $846(0-22)$ & $362(1-13)$ & 0.018 \\
\hline $\begin{array}{l}\text { Total Gr3-4 (range per } \\
\text { patient) }\end{array}$ & $57(0-4)$ & $47(0-4)$ & $10(0-2)$ & 0.016 \\
\hline $\begin{array}{l}\text { Total nr of events (range per } \\
\text { patient) }\end{array}$ & $1265(1-25)$ & $893(1-25)$ & $372(1-13)$ & 0.008 \\
\hline \multicolumn{5}{|c|}{$\begin{array}{l}\text { CT; computed tomography, SMI; skeletal muscle index, SD; standard deviation, Gr; grade, N; } \\
\text { number }\end{array}$} \\
\hline
\end{tabular}


Figure 2. Overall survival curve

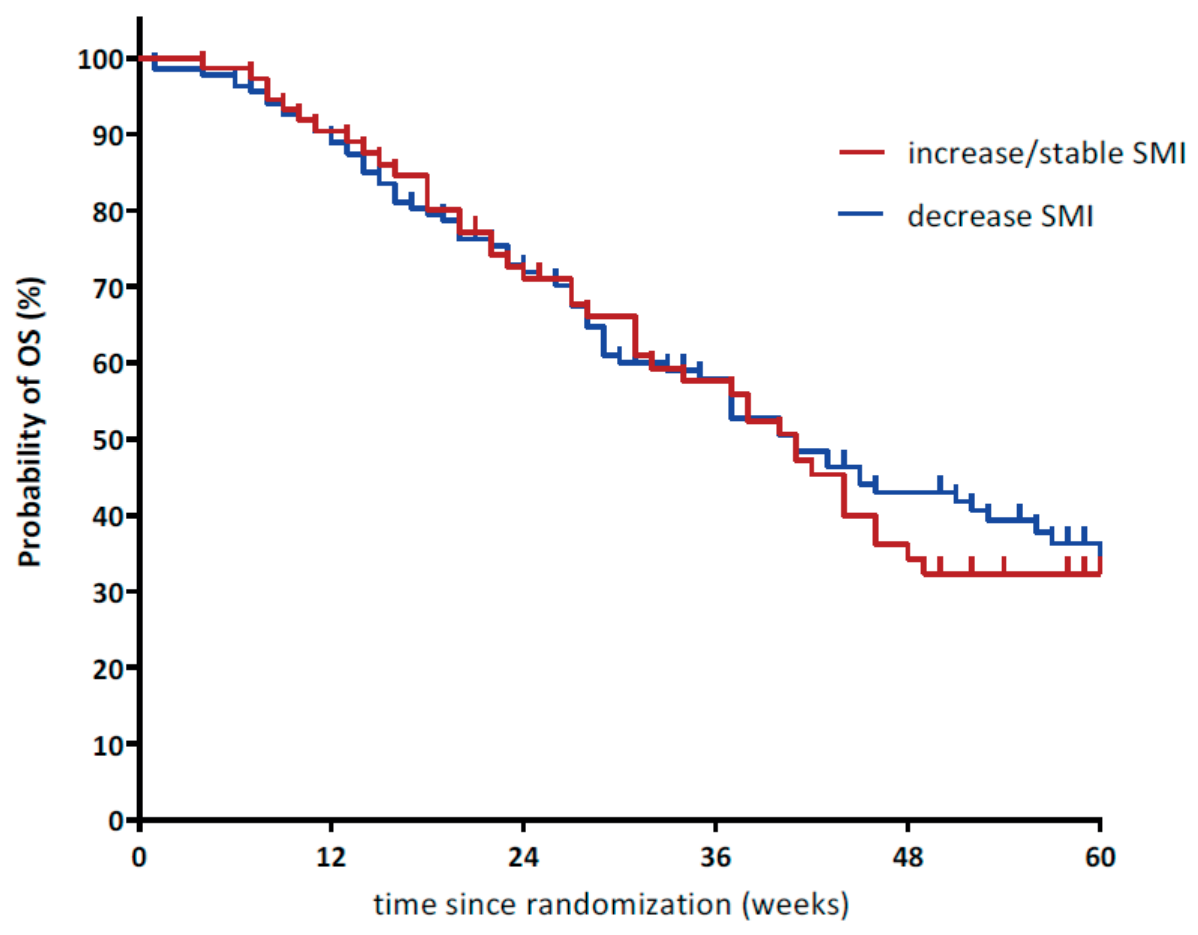

\begin{tabular}{llll}
\hline P-value & $\mathbf{0 . 7 6 4}$ & & \\
\hline$\Delta$ SMI: $-\mathbf{2}$ & Total $\mathbf{N}$ & $\mathbf{N}$ of events & $\%$ \\
\hline$\Delta$ SMI $>-2$ & 74 & 43 & 58 \\
$\Delta$ SMI $=<-2$ & 138 & 73 & 53 \\
Overall & 212 & 116 & 55 \\
\hline
\end{tabular}

Figure 2 Kaplan-Meier survival analysis. SMI; skeletal muscle index, plotted is an increase vs. decrease in SMI and the association with overall survival. 
Figure 3. Recurrence-free survival curve

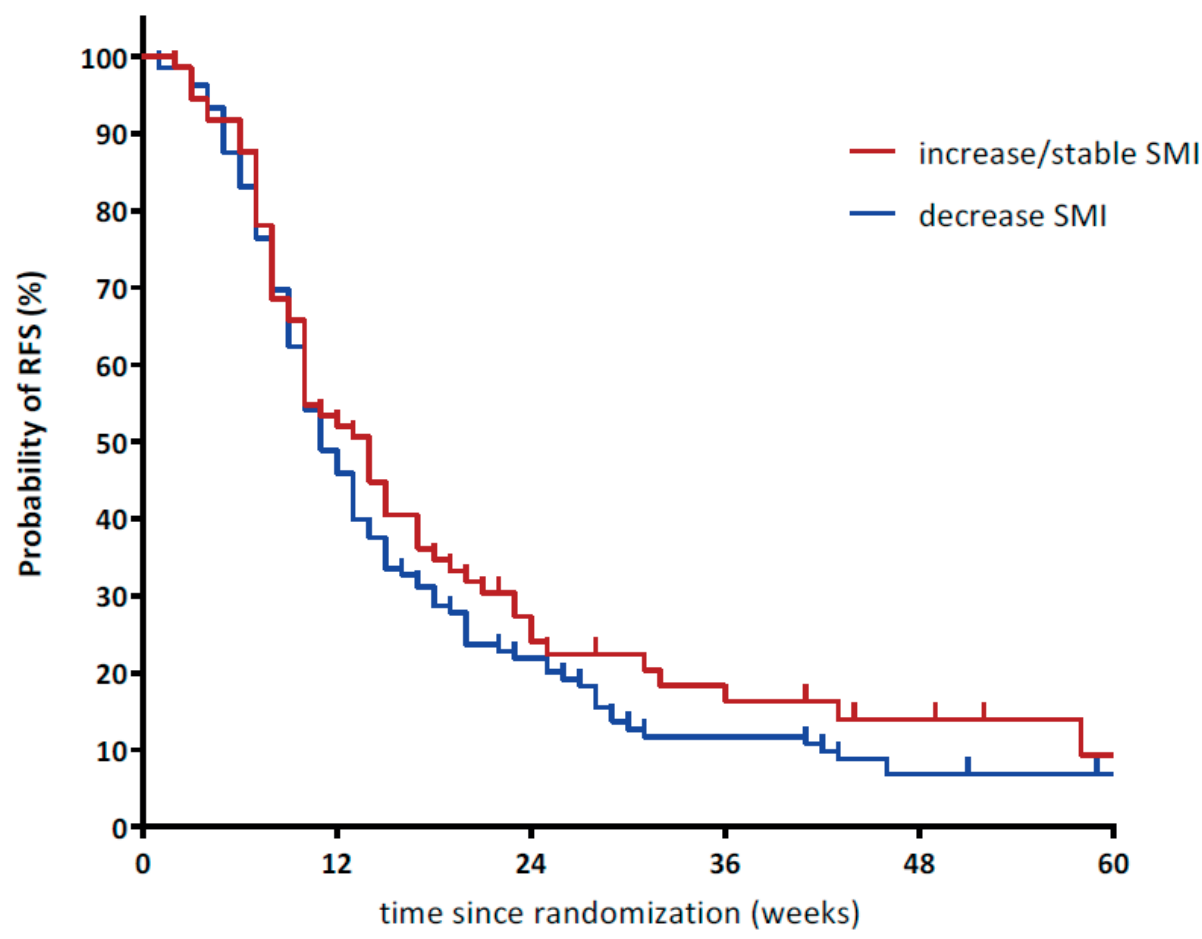

\begin{tabular}{llll}
\hline P-value & $\mathbf{0 . 2 9 7}$ & & \\
\hline $\boldsymbol{\Delta S M I :}-\mathbf{2}$ & Total $\mathbf{N}$ & $\mathbf{N}$ of events & $\%$ \\
\hline $\boldsymbol{\Delta} \mathbf{S M I}>-\mathbf{2}$ & 74 & 61 & 82 \\
$\boldsymbol{\Delta S M I}=<-2$ & 138 & 120 & 87 \\
Overall & 212 & 181 & 85 \\
\hline
\end{tabular}

Figure 3 Kaplan-Meier survival analysis. SMI; skeletal muscle index, plotted is an increase vs. decrease in SMI and the association with recurrence free survival. 


\section{Discussion}

The primary goal of this study was to validate the previously published finding that a decline in SMI during NACT in ovarian cancer patients was associated with worse OS [13]. Although a loss of skeletal muscle mass was detected in the present cohort, inclusion criteria allowed only for randomization of patients with prognostically favorable characteristics such as good response to chemotherapy or complete- or optimal cytoreduction. This selection of patients might have prevented validation of the results as published by Rutten et.al. Nevertheless, a strong association between decreasing SMI and adverse events was found.

Rutten et al. showed that ovarian cancer patients with a decrease in SMI (>2\%/100 days) had a significant $(p=0.004)$ survival disadvantage (median OS $916 \pm 99$ days) as opposed to patients who showed stable or increased SMI (median OS 1431 \pm 470 days) [13]. The patient cohort presented by Rutten et al. is essentially different regarding a number of aspects: 1) patients were older (mean age 60.9 [present] vs. 66.5 [Rutten et al.]), 2) had higher FIGO stage (III [present] vs. III-IV [Rutten et al.]), 3) received more chemotherapy between CT-scans (2 [present] vs. 3 cycles [Rutten et al.]), and 4) had worse outcome of CRS (68\% [present] vs. 45.5\% [Rutten et al.] patients with complete debulking surgery). The present study included patients under the age of 76, who were fit for major surgery (ASA1-2 and WHO performance score 0-2), had adequate organ function, and had only FIGO stage III disease[17]. In addition, only patients with a complete or partial response, or stable disease after two cycles, were selected. However, the mean decrease of SMI in both cohorts was quite similar $(5.9 \% / 100$ days present cohort vs. 5.2\%/100 days in the Rutten et al. cohort).

During NACT, patients with a decrease in SMI had a significantly higher number of toxicities of any grade (6.7, SD: 4.2 vs. 5.3; SD: $3.1, p=0.008$ ) (Table 3 ). This might indicate that skeletal muscle depletion is a measure of clinical fitness which impacts the patients' ability to receive treatment and thereby affects survival, rather than being an independent prognostic variable. Earlier findings already indicated that increased toxicity from chemotherapy is related to lower lean body mass, which results in reduced volume of distribution, protein binding, metabolism, and clearance of drugs[20]. The group of patients who lost skeletal muscle during chemotherapy had a slightly higher baseline BMI (20.9, SD 3.6) compared to the group of patients with stable/increased muscle mass (19.7, SD 3.1). It is not clear whether this slightly higher baseline BMI contributes to a greater decrease in skeletal muscle mass.[21]

The association between a decrease in SMI and outcome that was previously described[13] may be attributed to the fact that relatively frail patients were studied that did not meet the inclusion criteria for the OVHIPEC trial, but had a poor outcome due to adverse events, dose modifications or incomplete surgery. The strong relationship with reported toxicities in the current study is in line with this explanation. One of the main shortcomings of the current survival analysis is that it was not powered for analysis of OS/RFS, or for any subgroup analyses. All participants in the OVHIPEC trial were included for analysis with the assumption that sarcopenic patients were evenly distributed over both treatment arms. However, slightly more patients were treated with HIPEC in the group with a decrease in SMI. Multivariate analyses for treatment arm (HIPEC vs. no HIPEC) and for delta SMI were performed to evaluate the independent effect of deltaSMI without the potential mask by treatment with HIPEC (supplementary table S3 and S4). Skeletal muscle mass was not associated with recurrencefree and overall survival in multivariate analysis. Due to its prospective nature, this cohort is 
the most homogeneous described to date, and important confounders for outcome (FIGO stage, performance score, and surgical treatment) which were present in previous studies have been eliminated. The strict inclusion criteria in the current study make it difficult to generalize our findings to the general ovarian cancer patient population. Assessment of SMI was performed according to accepted methods $[10,22,23]$. The effect of a decrease in SMI was assessed independently of treatment with HIPEC in multivariate analysis. Since a survival disadvantage was not detected for patients with skeletal muscle mass loss, it is expected that optimal resection of the tumor is of greater importance for OS than adverse body composition. Based on previous cohorts, the association between adverse body composition and OS might still be of importance in older patients, or patients in whom complete resection of the tumor is not feasible. Due to the nature of the selected cohort, however, these patients were not analyzed[14, 15, 24, 25]. Multivariate analyses which take into account important prognostic variables such as treatment response, completeness of surgery, and toxicities could reveal if a decrease in body composition might be an independent predictor in other cohorts.

\section{Conclusion}

A change in SMI during 2 cycles of NACT was not associated with outcome within a large cohort of patients included in the OVHIPEC trial. In the current population, with relatively good prognostic characteristics, a loss of skeletal muscle mass was not an independent predictor for survival. In this selection of patients, we observed a significant association of sarcopenia with reported toxicities. Whether loss of skeletal muscle mass is related to patient performance status, toxicity or treatment burden should be confirmed in future analyses. 


\section{Supplementary material}

\section{Supplementary table 1}

\begin{tabular}{lrrl}
\hline & Mean (SD) & \multicolumn{1}{l}{ Range } & \\
\hline Time between Scan 1 and start & $2.92(1.88)$ & $0-9$ & Nr. of weeks prior to \\
NACT & & & NACT \\
Time between Scan 1 and iCRS & $13.98(2.24)$ & $9-22$ & Nr.of weeks prior to iCRS \\
Time between start NACT and & $4.89(1.20)$ & $3-9$ & Nr. of weeks after NACT \\
Scan 2 & & & \\
Time between Scan 2 and iCRS & $5.34(1.37)$ & $2-10$ & Nr. of weeks prior to iCRS \\
Time between Scan 1 and Scan 2 & $60.55(16.91)$ & $31-121$ & Nr of days between scan \\
& & & $1-2$ \\
\hline
\end{tabular}

Supplementary table 2

\begin{tabular}{lrrrrrr}
\hline & $\begin{array}{l}\text { Media } \\
\text { n }\end{array}$ & Mean & SE & SD & \multicolumn{1}{l}{ Range } & \multicolumn{1}{l}{$\begin{array}{l}\text { 33.33 } \\
\text { percentile }\end{array}$} \\
\hline SMI1 (N=212) & 39.49 & 39.55 & 0.37 & 5.42 & $27.54-57.93$ & 37.27 \\
SMI2 (N=212) & 38.31 & 38.08 & 0.34 & 4.98 & $26.44-55.40$ & 35.79 \\
$\begin{array}{l}\text { DeltaSMI per 100 } \\
\text { days (N=212) }\end{array}$ & $-5.42 \%$ & $-5.91 \%$ & 0.81 & 11.77 & $-31.61 \%-46.93 \%$ & \\
& & & & $\%$ & & \\
HU1 (N=220) & 35.87 & 36.08 & 0.53 & 7.91 & $17.91-56.60$ & 31.92 \\
HU2 (N=221) & 36.43 & 36.59 & 0.51 & 7.65 & $17.91-59.40$ & 32.78 \\
\hline
\end{tabular}

\section{Supplementary table 3}

Multivariable cox regression analyses for effect on overall survival

\begin{tabular}{l|lll|lll}
\hline Risk factor & Univariate & & & Multivariate & & \\
& HR & $95 \%-\mathrm{Cl}$ & $\mathrm{p}$-value & HR & $95 \%-\mathrm{Cl}$ & $\mathrm{p}$-value \\
\hline $\boldsymbol{\Delta S M I}$ & 1.059 & $0.726-$ & 0.766 & 0.987 & $0.674-$ & 0.948 \\
& & 1.544 & & & 1.447 & \\
Treatment & 0.668 & $0.462-$ & 0.032 & 0.667 & $0.459-$ & 0.033 \\
arm & & 0.965 & & & 0.968 & \\
\hline
\end{tabular}

HR; hazard ratio, 95\%-Cl; 95\% confidence interval, SMI; skeletal muscle index 


\section{Supplementary table 4}

Multivariable cox regression analyses for effect on recurrence-free survival

\begin{tabular}{l|lll|lll}
\hline Risk factor & Univariate & & & Multivariate & & \\
& HR & $95 \%-\mathrm{Cl}$ & $\mathrm{p}$-value & HR & $95 \%-\mathrm{Cl}$ & p-value \\
\hline $\boldsymbol{\Delta S \mathrm { SMI }}$ & 0.853 & $0.627-$ & 0.314 & 0.801 & $0.586-$ & 0.165 \\
& & 1.162 & & & 1.095 & \\
Treatment & 0.697 & $0.550-$ & 0.016 & 0.675 & $0.501-$ & 0.010 \\
arm & & 0.935 & & & 0.908 & \\
\hline
\end{tabular}

HR; hazard ratio, 95\%-Cl; 95\% confidence interval, SMI; skeletal muscle index

\section{Supplementary table 5}

Grade III and grade IV toxicity in both groups

\begin{tabular}{|c|c|c|}
\hline & $\begin{array}{l}\text { SMI decrease }>2 \% \\
N=133\end{array}$ & $\begin{array}{l}\text { SMI stable or SMI } \\
\text { increase } \\
\mathrm{N}=70\end{array}$ \\
\hline Pulmonary embolism & $86 \%$ & $00 \%$ \\
\hline Leukocytopenia/neutropenia & $65 \%$ & $23 \%$ \\
\hline Cardiac symptoms & $43 \%$ & $11 \%$ \\
\hline Peripheral neuropathy & $43 \%$ & $00 \%$ \\
\hline Abdominal pain & $32 \%$ & $11 \%$ \\
\hline Renal insufficiency & $32 \%$ & $00 \%$ \\
\hline Fatigue & $22 \%$ & $11 \%$ \\
\hline Abdominal distension (ascites) & $22 \%$ & $00 \%$ \\
\hline Diarrhea & $22 \%$ & $00 \%$ \\
\hline Dyspnea & $22 \%$ & $00 \%$ \\
\hline Ileus & $22 \%$ & $00 \%$ \\
\hline Thrombo-embolic event & $22 \%$ & $00 \%$ \\
\hline Infection & $11 \%$ & $23 \%$ \\
\hline Thrombocytopenia & $11 \%$ & $23 \%$ \\
\hline Constipation & $11 \%$ & $00 \%$ \\
\hline Dyspareunia & $11 \%$ & $00 \%$ \\
\hline Liver function anormality & $11 \%$ & $00 \%$ \\
\hline Loss of concentration & $11 \%$ & $00 \%$ \\
\hline Syncope & $11 \%$ & $00 \%$ \\
\hline Allergic reaction to paclitaxel & $0 \quad 0 \%$ & $11 \%$ \\
\hline Total & 47 & 10 \\
\hline
\end{tabular}


Supplementary Figure 1 ROC curve for recurrence-free survival and different outcome variables

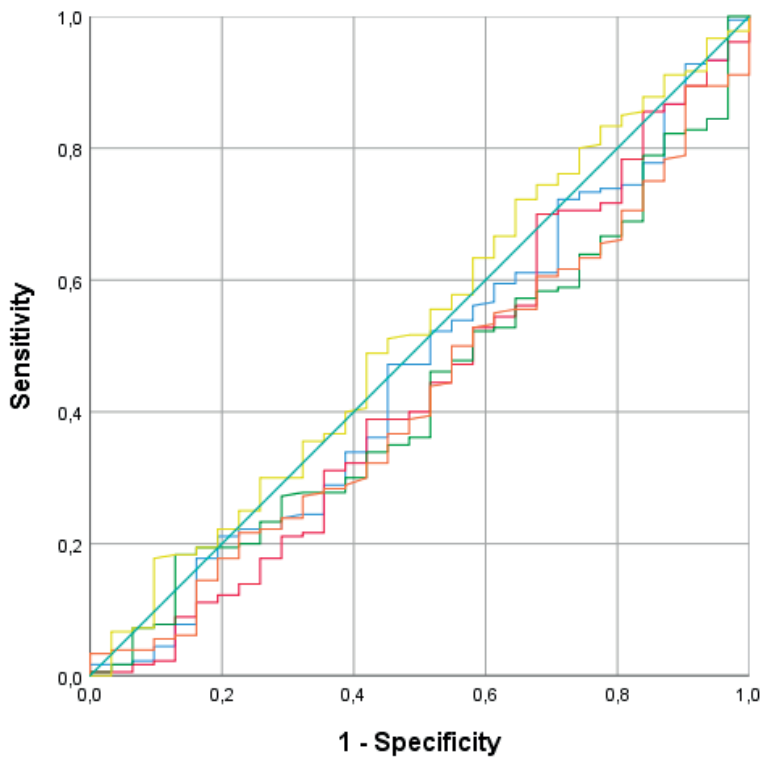

Source of the Curve

SMI. 1

- SMI.2

- DeltaSMI100

- meanHU.1

- meanHU.2

- Reference Line

Supplementary Figure $\mathbf{2}$ ROC curve for overall survival and different outcome variables

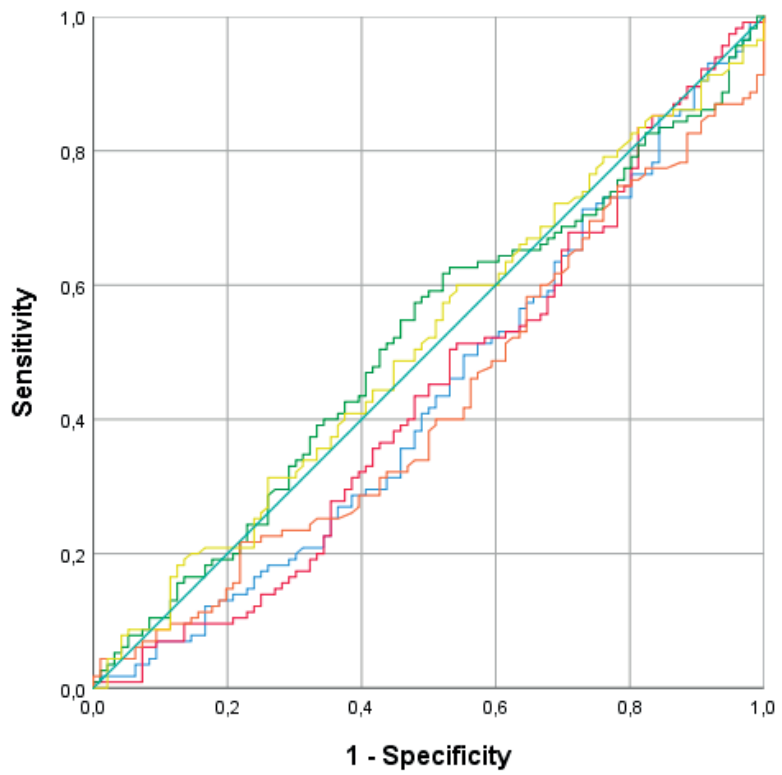

Source of the Curve

- SMI.1

- SMI.2

- DeltaSMI100

meanHU.1

meanHU.2

- Reference Line 


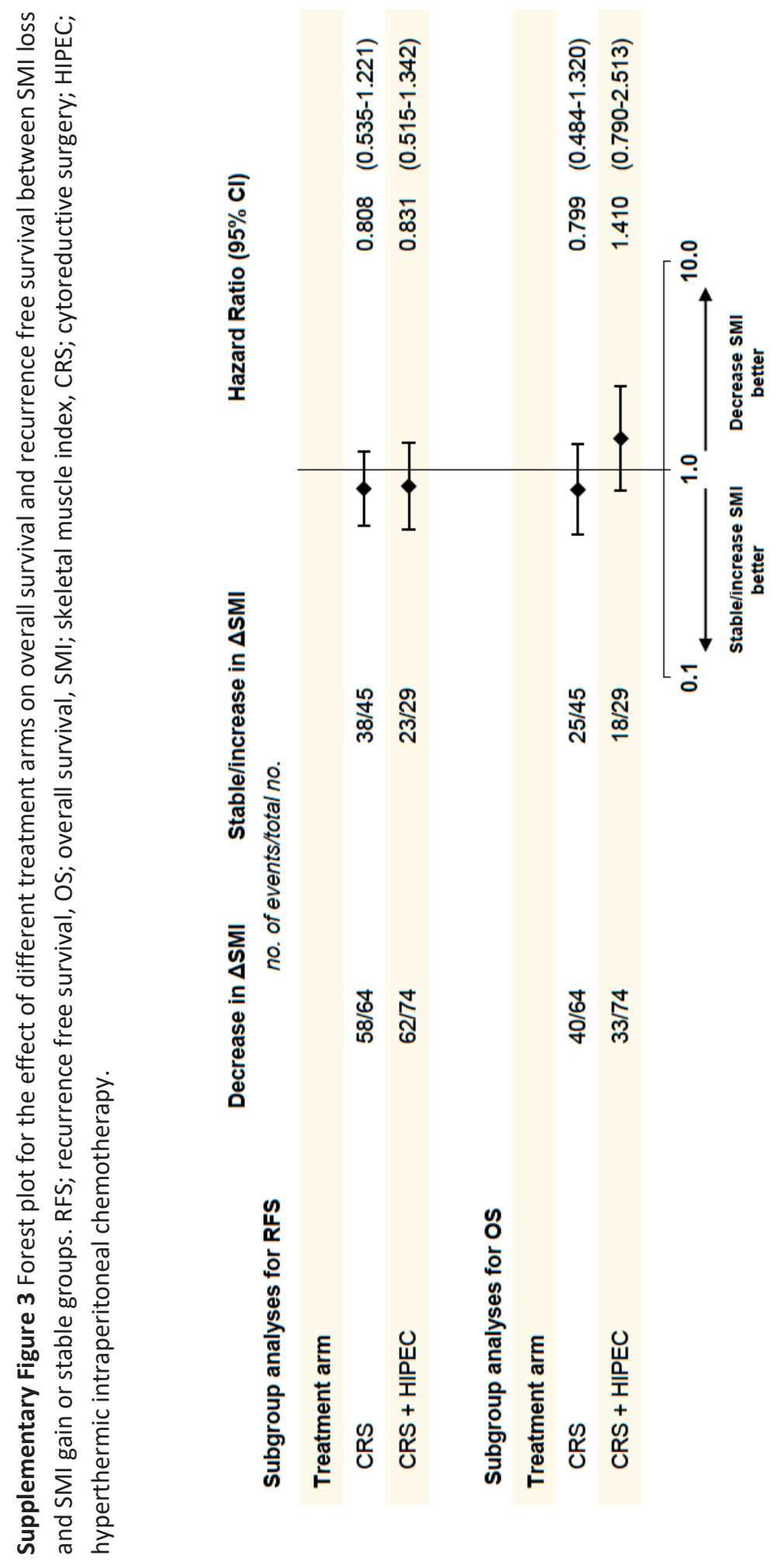




\section{References}

1. Heintz, A.P., et al., Carcinoma of the ovary. FIGO 26th Annual Report on the Results of Treatment in Gynecological Cancer. Int J Gynaecol Obstet, 2006. 95 Suppl 1: p. S16192.

2. Narod, S., Can advanced-stage ovarian cancer be cured? Nat Rev Clin Oncol, 2016. 13(4): p. 255-61.

3. Timmermans, M., et al., No improvement in long-term survival for epithelial ovarian cancer patients: A population-based study between 1989 and 2014 in the Netherlands. European Journal of Cancer, 2017. 88: p. 31-37.

4. van der Burg, M.E., et al., The effect of debulking surgery after induction chemotherapy on the prognosis in advanced epithelial ovarian cancer. Gynecological Cancer Cooperative Group of the European Organization for Research and Treatment of Cancer. N Engl J Med, 1995. 332(10): p. 629-34.

5. Vergote, I., et al., Neoadjuvant chemotherapy or primary surgery in stage IIIC or IV ovarian cancer. N Engl J Med, 2010. 363(10): p. 943-53.

6. Wright, A.A., et al., Neoadjuvant chemotherapy for newly diagnosed, advanced ovarian cancer: Society of Gynecologic Oncology and American Society of Clinical Oncology Clinical Practice Guideline. Gynecol Oncol, 2016. 143(1): p. 3-15.

7. Chang, S.J., et al., Survival impact of complete cytoreduction to no gross residual disease for advanced-stage ovarian cancer: a meta-analysis. Gynecol Oncol, 2013. 130(3): p. 493-8.

8. Timmermans, M., et al., Interval between debulking surgery and adjuvant chemotherapy is associated with overall survival in patients with advanced ovarian cancer. Gynecol Oncol, 2018. 150(3): p. 446-450.

9. Fearon, K., et al., Definition and classification of cancer cachexia: an international consensus. Lancet Oncol, 2011. 12(5): p. 489-95.

10. Mourtzakis, M., et al., A practical and precise approach to quantification of body composition in cancer patients using computed tomography images acquired during routine care. Appl Physiol Nutr Metab, 2008. 33(5): p. 997-1006.

11. Prado, C.M., et al., The association between body composition and toxicities from the combination of Doxil and trabectedin in patients with advanced relapsed ovarian cancer. Appl Physiol Nutr Metab, 2014. 39(6): p. 693-8.

12. Yoshikawa, T., et al., Psoas muscle volume as a predictor of peripheral neurotoxicity induced by primary chemotherapy in ovarian cancers. Cancer Chemother Pharmacol, 2017. 80(3): p. 555-561.

13. Rutten, I.J., et al., Loss of skeletal muscle during neoadjuvant chemotherapy is related to decreased survival in ovarian cancer patients. J Cachexia Sarcopenia Muscle, 2016. 7(4): p. 458-66.

14. Rutten, I.J., et al., The influence of sarcopenia on survival and surgical complications in ovarian cancer patients undergoing primary debulking surgery. Eur J Surg Oncol, 2017. 43(4): p. 717-724.

15. Bronger, H., et al., Sarcopenia in Advanced Serous Ovarian Cancer. International Journal of Gynecological Cancer, 2017. 27(2): p. 223-232.

16. Silva de Paula, N., et al., Sarcopenia and Skeletal Muscle Quality as Predictors of Postoperative Complication and Early Mortality in Gynecologic Cancer. International Journal of Gynecological Cancer. 28(2): p. 412-420. 
17. van Driel, W.J., et al., Hyperthermic Intraperitoneal Chemotherapy in Ovarian Cancer. N Engl J Med, 2018. 378(3): p. 230-240.

18. Mitsiopoulos, N., et al., Cadaver validation of skeletal muscle measurement by magnetic resonance imaging and computerized tomography. J Appl Physiol (1985), 1998. 85(1): p. 115-22.

19. Rustin, G.J., et al., Definitions for response and progression in ovarian cancer clinical trials incorporating RECIST 1.1 and CA 125 agreed by the Gynecological Cancer Intergroup (GCIG). Int J Gynecol Cancer, 2011. 21(2): p. 419-23.

20. Hopkins, J.J. and M.B. Sawyer, A review of body composition and pharmacokinetics in oncology. Expert Rev Clin Pharmacol, 2017. 10(9): p. 947-956.

21. Martin, L., et al., Cancer cachexia in the age of obesity: skeletal muscle depletion is a powerful prognostic factor, independent of body mass index. J Clin Oncol, 2013. 31(12): p. $1539-47$.

22. Rutten, I.J.G., et al., Psoas muscle area is not representative of total skeletal muscle area in the assessment of sarcopenia in ovarian cancer. J Cachexia Sarcopenia Muscle, 2017. 8(4): p. 630-638.

23. Baracos, V.E., Psoas as a sentinel muscle for sarcopenia: a flawed premise. J Cachexia Sarcopenia Muscle, 2017. 8(4): p. 527-528.

24. Kumar, A., et al., Muscle composition measured by CT scan is a measurable predictor of overall survival in advanced ovarian cancer. Gynecologic Oncology, 2016. 142(2): p. 311-316.

25. Ataseven, B., et al., Skeletal Muscle Attenuation (Sarcopenia) Predicts Reduced Overall Survival in Patients with Advanced Epithelial Ovarian Cancer Undergoing Primary Debulking Surgery. Annals of Surgical Oncology, 2018. 25(11): p. 3372-3379. 
Chapter 5 | 93 


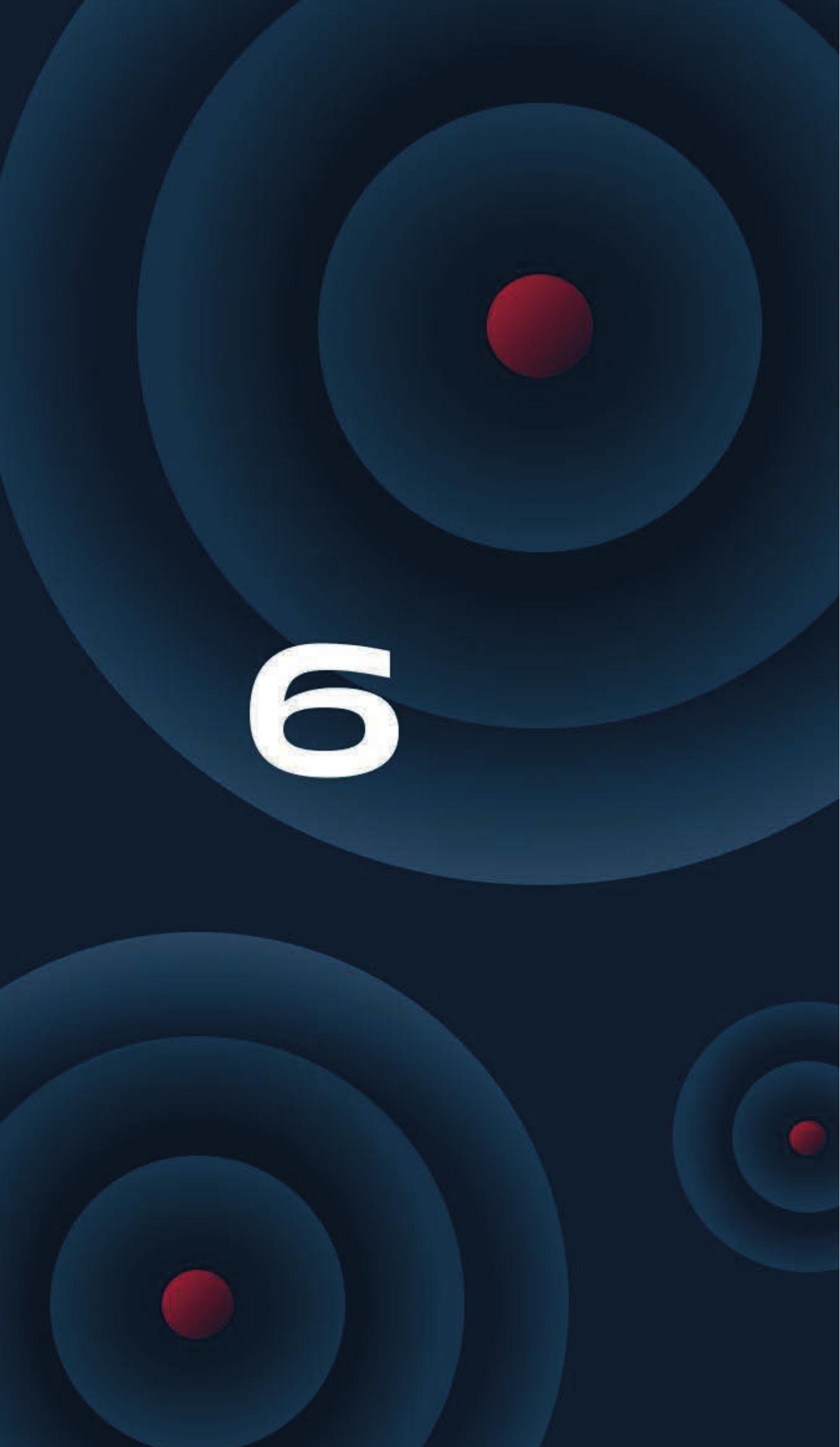




\section{Ovarian cancer ascites induces skeletal muscle wasting in vitro and reflects sarcopenia in patients}

Submitted to the Journal Of Cachexia, Sarcopenia and Muscle (2021)

Jorne Ubachs, Wouter van de Worp, Rianne Vaes, Kenneth Pasmans, Ramon Langen, Ruth Meex, Sandrina Lambrechts, Toon Van Gorp, Roy Kruitwagen, Steven Olde Damink, Sander Rensen 


\section{Abstract \\ Background}

Cachexia-associated skeletal muscle wasting or 'sarcopenia' is highly prevalent in ovarian cancer and contributes to poor outcome. Drivers of cachexia-associated sarcopenia in ovarian cancer remain elusive, underscoring the need for novel and better models to identify tumor factors inducing sarcopenia. We aimed to assess whether factors present in ascites of sarcopenic versus non-sarcopenic ovarian cancer patients differentially affect protein metabolism in skeletal muscle cells, and to determine if these effects are correlated to cachexia-related patient characteristics.

\section{Methods}

Fifteen patients with an ovarian mass and ascites underwent extensive physical screening focusing on cachexia-related parameters. Based on CT-based body composition imaging, six cancer patients were classified as sarcopenic and six were not; three patients with a benign condition served as an additional non-sarcopenic control group. Ascites was collected and concentrations of cachexia-associated factors were assessed by ELISA. Subsequently, ascites was used for in vitro exposure of $\mathrm{C} 2 \mathrm{C} 12$ myotubes followed by measurements of protein synthesis and breakdown by radioactive isotope tracing, qPCR-based analysis of atrophyrelated gene expression, and NF-kB activity reporter assays.

\section{Results}

C2C12 protein synthesis was lower after exposure to ascites from sarcopenic patients (sarcopenia $3.1 \pm 0.1 \mathrm{nmol} / \mathrm{h} / \mathrm{mg}$ protein vs. non-sarcopenia $5.5 \pm 0.2 \mathrm{nmol} / \mathrm{h} / \mathrm{mg}$ protein, $\mathrm{p}<0.01$ ), and protein breakdown rates tended to be higher (sarcopenia $31.2 \pm 5.2 \%$ vs. nonsarcopenia 20.9 $\pm 1.9 \%, p=0.08$ ). Ascites did not affect MuRF-1, Atrogin-1, or REDD1 expression of $\mathrm{C} 2 \mathrm{C} 12$ myotubes, but NF-KB activity was specifically increased in cells exposed to ascites from sarcopenic patients (sarcopenia; 2.2 \pm 0.4 -fold compared to control vs. non-sarcopenia $1.2 \pm 0.2$-fold compared to control, $p=0.01$ ). Protein synthesis and breakdown correlated with NF-KB activity $\left(r_{s}=-0.60, p=0.03\right.$ and $r_{s}=0.67, p=0.01$, respectively). The skeletal muscle index of the ascites donors was correlated to both in vitro protein synthesis $\left(r_{s}=0.70, p=0.005\right)$ and protein breakdown rates $\left(r_{s}=-0.57, p=0.04\right)$.

\section{Conclusion}

Ascites of sarcopenic ovarian cancer patients induces pronounced skeletal muscle protein metabolism changes in $\mathrm{C} 2 \mathrm{C} 12$ cells that correlate with clinical muscle measures of the patient and that are characteristic of cachexia. The use of ascites offers a new experimental tool to study the impact of both tumor-derived and systemic factors in various cachexia model systems, enabling identification of novel drivers of tissue wasting in ovarian cancer. 


\section{Introduction}

Cachexia and skeletal muscle wasting are highly prevalent in patients with ovarian cancer and are associated with poor disease outcome.[1] The mechanisms underlying the development of cachexia in ovarian cancer remain elusive, although several lines of evidence have implicated tumor-derived compounds and their direct and indirect effects on skeletal muscle and adipose tissue.[2-5] In particular, skeletal muscle protein metabolism alterations induced by changes in Muscle RING-finger protein-1 (MuRF1), Atrogin-1, REDD-1 (regulated in development and DNA damage responses 1) [6, 7], and/or NF-kB activity [8] have been the focus of research.

About one third of all newly diagnosed ovarian cancer patients and almost all patients with recurrent disease accumulate ascites.[9] In the intraperitoneal cavity, small amounts of free fluids are produced by capillary membranes to allow for lubrication of serosal surfaces. Under physiological conditions, the vast majority of these fluids are reabsorbed by the lymphatic system. However, under influence of tumor-derived growth factors such as vascular endothelial growth factor, the peritumoral microvasculature becomes increasingly leaky. Furthermore, disseminated disease can cause obstruction of the lymphatic vessels. [10] This combination of increased build up and decreased reabsorption causes rapid accumulation of ascites fluid in the peritoneal cavity under malignant conditions.[11] Importantly, excessive accumulation of ascites has been associated with detrimental nutritional status in ovarian cancer patients.[12]

Ovarian cancer ascites is a complex reservoir of soluble factors and cell components which collectively provide a pro-inflammatory and tumor promoting microenvironment.[13] Interestingly, cytokine expression profiling of ascites from ovarian cancer patients revealed high levels of IL-6, IL-8, and Mcp-1[14], factors that have been suggested to promote tissue wasting in individuals with cachexia.[15] Furthermore, the concentration of cachexia-related inflammatory cytokines in ovarian cancer ascites has been shown to be significantly higher in comparison to the serum concentrations of the same patient.[16] Since ovarian cancer ascites is relatively easily accessible, generally present in large quantities, and contains a high concentration of tumor-derived compounds, it represents an attractive experimental tool to study the impact of ovarian cancer-derived factors on skeletal muscle physiology.

We hypothesized that factors present in ovarian cancer ascites from sarcopenic patients would induce protein metabolism disturbances characteristic of cachexia-associated sarcopenia in skeletal muscle cells. C2C12 skeletal muscle cells were exposed to ascites from well-phenotyped sarcopenic vs. non-sarcopenic patients with malignant or benign ovarian tumors, followed by analysis of protein synthesis and breakdown. We found that ascites from sarcopenic cancer patients decreased $\mathrm{C} 2 \mathrm{C} 12$ protein synthesis in correspondence with their degree of sarcopenia. 


\section{Methods}

\section{Patients and cachexia screening}

Between March 2017 and March 2018, fifteen consecutive patients with a suspected malignancy of the ovary, as indicated by CT-scan analysis and the presence of abdominal ascites, were prospectively enrolled at the Maastricht University Medical Centre+ (MUMC+). Patients were eligible for either primary cytoreductive surgery (CRS) or neo-adjuvant chemotherapy. Before start of the treatment, patients received a physical screening including assessment of handgrip strength, triceps skinfold assessment (TSA), upper arm circumference (UAC), and wrist circumference (WC). The physical screening was complemented with the Patient-Generated Subjective Global Assessment (PG-SGA), Mini Nutritional Assessment (MNA), and subjective assessment of fat, muscle, and fluid status. Patient reported weight loss was assessed over the past six months. Venous blood was drawn and concentrations of hemoglobin $(\mathrm{Hb})$, leukocytes (and differentiation), kidney function markers, liver function indicators, lipids, insulin, glucose, and acute phase proteins were assessed to characterize cancer cachexia and to identify possible promoters of sarcopenia.

For body composition analysis, one single axial slice of the abdominal CT scan at the third lumbar level was used. Standard Hounsfield unit ranges of -30 to +150 Hounsfield Units (HU) for skeletal muscle (SM), -190 to $-30 \mathrm{HU}$ for intramuscular adipose tissue (IMAT) and subcutaneous adipose tissue (SAT), and -150 to $-50 \mathrm{HU}$ for visceral adipose tissue (VAT) were used to demarcate tissue using SliceOmatic software (v5.0, Tomovision, Montreal, Canada). Following demarcation, surface areas were standardized by height to compute the skeletal muscle index (SMI) in $\mathrm{cm}^{2} / \mathrm{m}^{2}$. Skeletal muscle radiation attenuation (SMRA) was calculated using the mean HU values of SM. Patients with a malignancy $(n=12)$ were divided into a sarcopenic and a non-sarcopenic group based on their L3-SMI. The cut-off for sarcopenia was determined by one SD below the mean SMI (SMI $\left.39.1 \mathrm{~cm}^{2} / \mathrm{m}^{2}\right)$. [1] Three patients with a benign ovarian condition served as non-sarcopenic controls. This study was approved by the Medical Ethics Committee of Maastricht University Medical Centre+ and has been performed in accordance with the ethical standards laid down in the 1964 Declaration of Helsinki and all its revisions. All patients gave their informed consent prior to their inclusion in the study.

\section{Collection and analysis of ascites}

Ascites was collected either during an abdominal paracentesis or during primary CRS, but in all instances before any systemic treatment was started. Although more ascites was present, between $30 \mathrm{~mL}$ and $200 \mathrm{~mL}$ of ascites were collected for further analysis (see Table 1). After aspiration, the ascites was kept on ice before centrifugation for $10 \mathrm{~min}$ at 200x g. The supernatant was centrifuged again for $15 \mathrm{~min}$ at $350 \mathrm{x}$ g. Cell-free supernatant was aliquoted and stored at $-80 \mathrm{C}^{\circ}$. The ascites was processed under sterile conditions in a flow cabinet.

The concentrations of IL-6, IL-8, GDF-8, GDF-15, Tumor Necrosis Factor (TNF)- $\alpha$, IL-1 $\beta$, leukemia inhibitory factor, and Mcp-1 in ascites were quantified with enzyme-linked immunosorbent assays (R\&D systems, Minneapolis, USA) as per manufacturers protocol.

\section{C2C12 cell culture}

C2C12 murine myoblasts (American Type Culture Collection No. CRL1772, Manassas, VA) were cultured in growth medium (GM), composed of low glucose (1g/L) Dulbecco's Modified Eagle Medium (DMEM) (Gibco, Dublin, Ireland) supplemented with $10 \%(\mathrm{v} / \mathrm{v})$ fetal bovine serum 
(FBS) and $1 \%(\mathrm{v} / \mathrm{v})$ antibiotics (100 units/mL penicillin and $100 \mu \mathrm{g} / \mathrm{mL}$ streptomycin, GIBCO). Cells were maintained at $37^{\circ} \mathrm{C}$ and $5 \% \mathrm{CO}_{2}$ until $70-80 \%$ confluency was reached at which point the cells were passaged or used for experiments.

Depending on the experiment, cells were seeded at a density of $1.5 \times 10^{4} \mathrm{cell} / \mathrm{cm}^{2}$ on BD Matrigel-coated (Matrigel ${ }^{\circledR}$ Matrix Basement Membrane - Growth factor reduced, Corning) (1:50 in low glucose DMEM) cell culture plates (Eppendorf). Myoblasts were cultured for $24 \mathrm{~h}$ in GM. After 24h, differentiation was initiated after washing the cells with Dulbecco's phosphate-buffered saline (PBS) (Gibco, Dublin, Ireland) and switching the GM to differentiation medium (DM)[17], which consisted of high glucose (4.5 g/L) DMEM supplemented with $1 \%(\mathrm{v} / \mathrm{v})$ heat inactivated $\mathrm{FBS}\left(30 \mathrm{~min}\right.$ at $\left.56^{\circ} \mathrm{C}\right), 1 \%(\mathrm{v} / \mathrm{v})$ sodium pyruvate, and $0.5 \%(\mathrm{v} / \mathrm{v})$ antibiotics (50 units $/ \mathrm{mL}$ penicillin and $50 \mu \mathrm{g} / \mathrm{mL}$ streptomycin). DM was refreshed every $48 \mathrm{~h}$ for 5-6 days at which point the cells were used for experiments.

\section{Analysis of protein synthesis}

Fully differentiated myotubes were treated with ascites or indicated control compounds for $24 \mathrm{~h}$ in humidified conditions at $37^{\circ} \mathrm{C}$ and $5 \% \mathrm{CO}_{2}$. Ascites was diluted in DM ( $\left.25 \% \mathrm{v} / \mathrm{v}\right)$. Controls consisted of $100 \%$ DM, Hank's balanced salt solution (HBSS) in DM ( $25 \% \mathrm{v} / \mathrm{v}), 100 \mathrm{nM}$ insulin (Eli Lilly, Indianapolis, USA) in DM (positive control), or $10 \mu \mathrm{M}$ dexamethasone (Sigma) solubilized in absolute ethanol and diluted in DM (negative control). All compounds and media were pre-warmed to $37^{\circ} \mathrm{C}$ before they were added to the cells. Before treatment, the $\mathrm{C} 2 \mathrm{C} 12$ cells were washed twice with warm PBS. After treatment, cells were washed again with prewarmed PBS and incubated in DM containing 0,3mM L-phenylalanine (Sigma) and $0,1 \mu \mathrm{Ci} / \mathrm{mL}$ ${ }^{14} \mathrm{C}$-L-phenylalanine (PerkinElmer, Waltham, USA) for $8 \mathrm{~h}$. After incubation, cells were washed with ice cold PBS, and intracellular proteins were subsequently precipitated in $1 \mathrm{M}$ of perchloric acid (PCA) for $1 \mathrm{~h}$ at $4^{\circ} \mathrm{C}$. After washing, the cells were left overnight in $1 \mathrm{M}$ of sodium hydroxide $(\mathrm{NaOH})+1 \%$ sodium dodecylsulphate (SDS) solution. Subsequently, cells were scraped and protein concentrations in the lysates were measured with the BCA assay (Thermo Scientific, Waltham, USA). Protein lysates were suspended in Ultima Gold multi-purpose liquid scintillation cocktail (LSC) (Sigma) and ${ }^{14} \mathrm{C}$-L-phenylalanine incorporation was measured with a TriCarb 2910 low activity liquid scintillation analyzer (LALSA) (PerkinElmer, Waltham, USA). Protein synthesis was expressed as $\mathrm{nmol}{ }^{14} \mathrm{C}$-L-phenylalanine incorporated per hour of exposure per milligram of total protein ( $\mathrm{nmol} / \mathrm{h} / \mathrm{mg})$. (See supplementary material 2 ).

\section{Analysis of protein breakdown}

Fully differentiated C2C12 myotubes were pre-treated with $0.2 \mu \mathrm{Ci} / \mathrm{mL} 14 \mathrm{C}$-L-phenylalanine and $0.3 \mathrm{mM}$ unlabeled L-phenylalanine in DM for $24 \mathrm{~h}$. The pre-treatment was followed by a $2 \mathrm{~h}$ chase period in which the cells were exposed to $0.3 \mathrm{mM}$ unlabeled L-phenylalanine in DM to prevent the reincorporation of labeled phenylalanine released from degraded protein during protein synthesis. The chase period was followed by a $24 \mathrm{~h}$ period of treatment with experimental conditions. The same experimental conditions and controls as for the synthesis experiment were used for analysis of protein breakdown. After treatment, the culture medium was collected, proteins in the medium were precipitated with $1 \mathrm{M}$ PCA, and, after centrifugation, the supernatant was added to LSC and counted (reading A). The precipitates were resuspended and solubilized by adding $1 \mathrm{M} \mathrm{NaOH}+1 \%$ SDS, and after incubation at $37 \mathrm{Co}$ for $2 \mathrm{~h}$, they were added to LSC and counted (reading $\mathrm{B}$ ). The cells in the plate were washed with PBS, solubilized with $1 \mathrm{M} \mathrm{NaOH}+1 \%$ SDS, followed by overnight precipitation in $1 \mathrm{M} \mathrm{PCA}$ at $4 \circ \mathrm{OC}$. Cells were scraped and protein concentrations were measured with the BCA assay. 
Protein samples were suspended in LSC and 14C-L-phenylalanine incorporation was measured with a LALSA (reading C). Incorporated protein was expressed as nmol 14C-L-phenylalanine incorporated per hour of exposure per milligram of total intracellular protein $(\mathrm{nmol} / \mathrm{h} / \mathrm{mg})$. Breakdown was calculated as reading A/(reading A+ reading $B+$ reading C)*100, and expressed as \% breakdown (see supplementary material 3).

\section{Analysis of signaling pathways}

C2C12 cells stably transfected with a $6 \mathrm{~KB}-\mathrm{TK}$ luciferase plasmid (NF-KB reporter) were used for the assessment of NF-KB transcriptional activity, as previously described.[8] After full differentiation, the cells were treated with ascites in DM ( $25 \% \mathrm{v} / \mathrm{v}$, experimental condition) or with $1 \mathrm{nmol}$ TNF- $\alpha$ in DM (positive control) or HBSS (25\% v/v, control condition) for $8 \mathrm{~h}$. $\mathrm{NaHCO} 3$ and 4-(2-hydroxyethyl)-1-piperazineethanesulfonic acid (HEPES) (Sigma, St. Louis, USA) were added to HBSS for buffering capacities. All compounds and media were prewarmed to $370 \mathrm{O}$ before they were added to the cells. Cells were lysed with $1 \mathrm{x}$ lysis buffer (Promega, Madison, USA) (20\% v/v in 1x DPBS) and protein lysates were scraped and stored at $-80 \circ \mathrm{C}$. Protein concentrations were measured with a BCA assay and luciferase activity was determined according to the manufacturer's instructions (Promega). Luciferase activity was normalized for protein concentration and expressed as a fold change of the benign control.

For analysis of MURF1, Atrogin-1, and REDD-1 gene expression, fully differentiated myotubes were treated with ascites in DM ( $25 \% \mathrm{v} / \mathrm{v}$, experimental condition) or with HBSS in DM ( $25 \%$ $\mathrm{v} / \mathrm{v}$, control condition) or with $100 \% \mathrm{HBSS}$ as positive control for $24 \mathrm{~h}$. RNA was isolated with TRIzol Reagent (Thermo Fisher, Waltham, USA) and RNA concentration was determined with a DS-11 microvolume spectrophotometer (DeNovix, Wilmington, USA). RNA was reverse transcribed using the SensiFast cDNA Synthesis Kit according to the manufacturer's instructions (Bioline $\mathrm{GmbH}$, Germany). cDNA was amplified with the SensiMix SYBR Hi-Rox Kit (Bioline) on a Lightcycler 480 PCR platform (Roche, Almere, The Netherlands). LinRegPCR 11.0 software (Amsterdam, Netherlands) was used to assess PCR efficiency. Expression of the genes of interest was normalized with a correction factor derived from several reference genes (cyclophilin A, $\beta$-2-microglobulin) using geNorm (qBase+, Belgium). Results are expressed as a fold change of the benign control. See supplementary material 1 for primer sequences.

\section{Statistical analysis}

Baseline data between all groups were compared with one-way analysis of variance (ANOVA) or Kruskal-Wallis tests where applicable. Relationships between continuous variables were tested with Spearman correlation coefficients. A correlation matrix was used to visualize these correlations using R 3.6.1 for Microsoft Windows. For all other statistical analyses, SPSS v23.0 (IBM Corp, Chicago, IL) was used. GraphPad Prism v. 5.03 was used to make the box plots. Correction for multiple testing with the Kruskal-Wallis test was only applied to the experimental conditions for in vitro outcomes. P values $<0.05$ were considered statistically significant. 


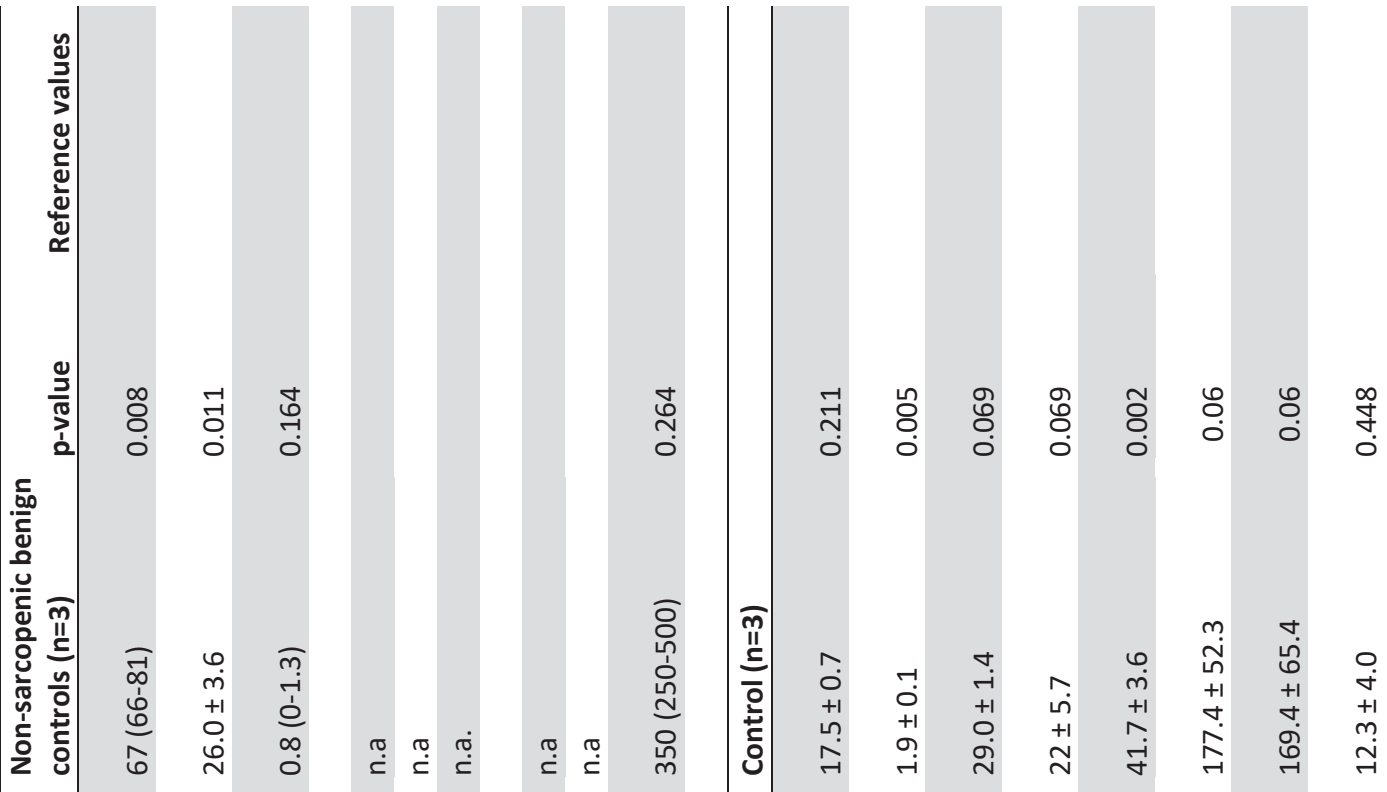

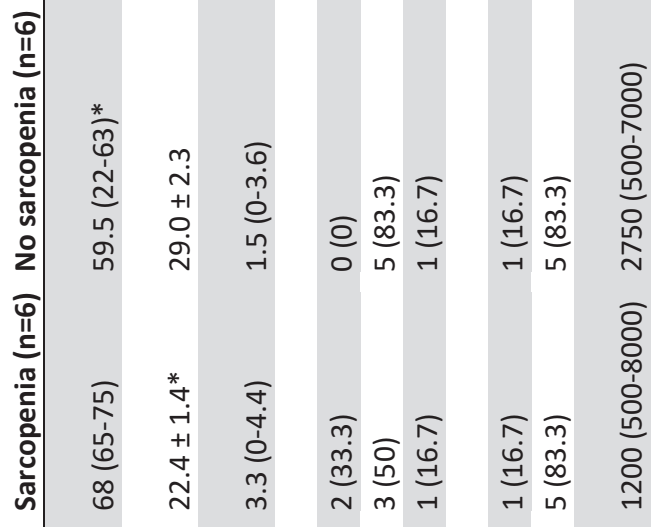

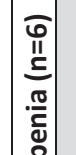

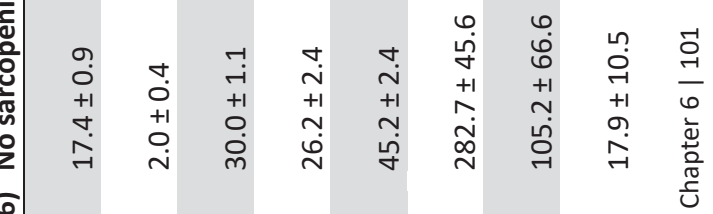

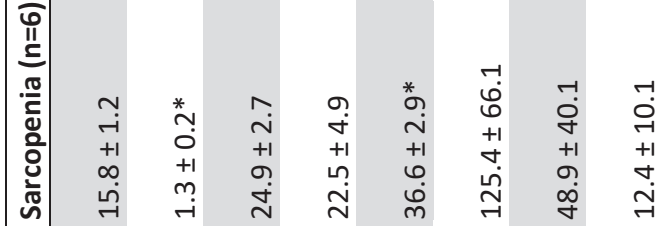

$\stackrel{\text { In }}{\text { II }}$

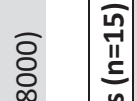

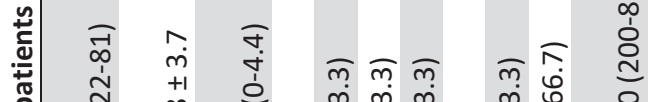

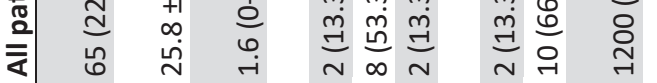

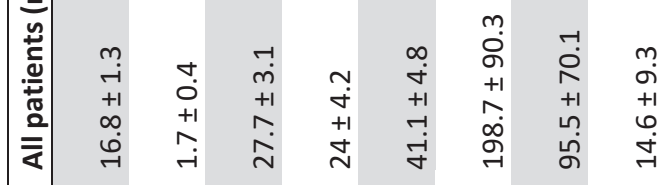

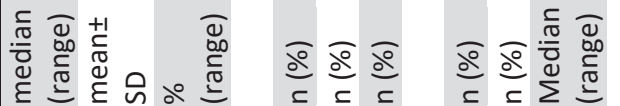

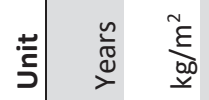

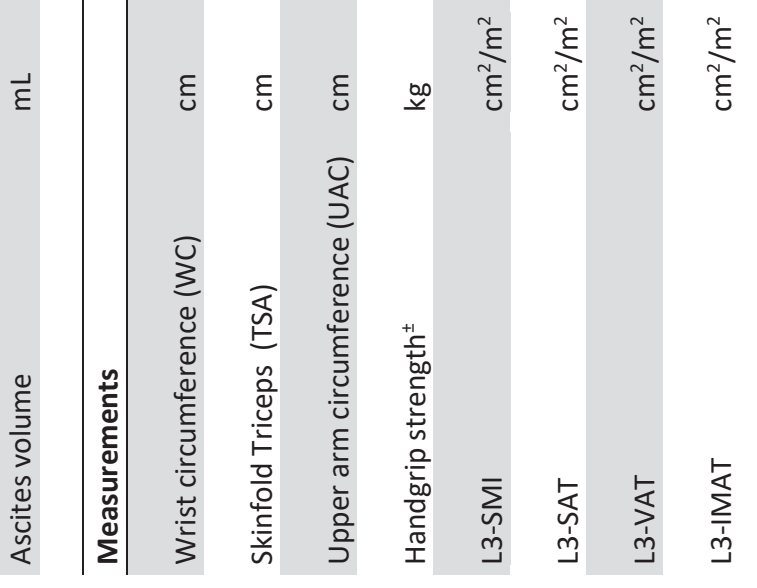




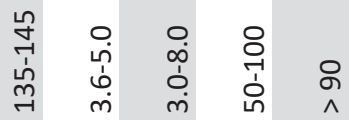

$\stackrel{\substack{0 \\ 0}}{0}$

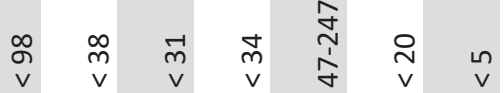

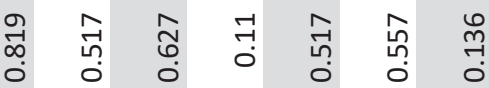

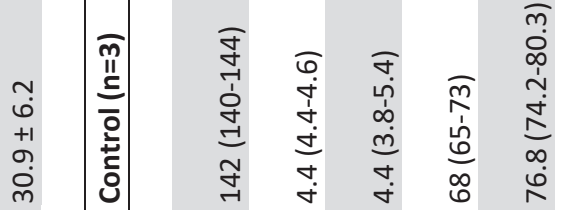

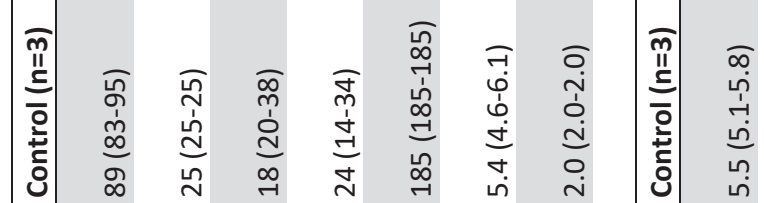

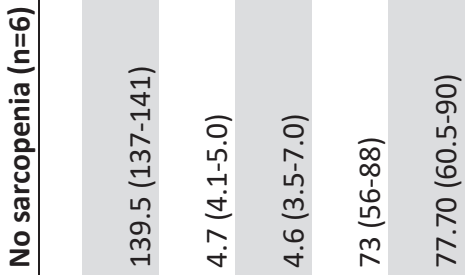

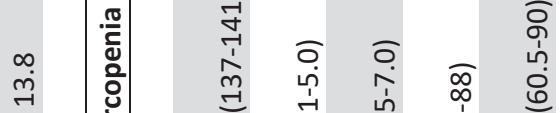

+ +

我

离

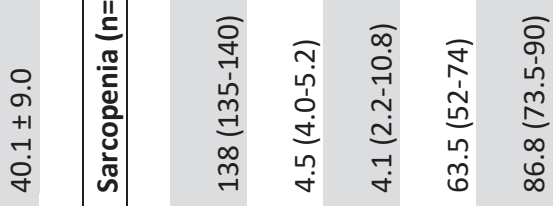

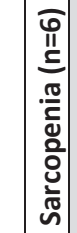

)

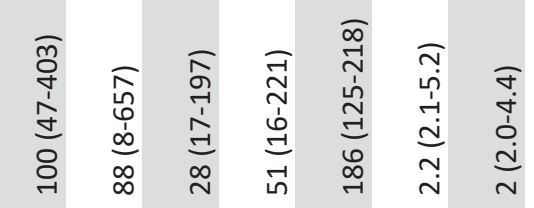

预

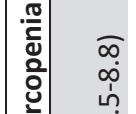

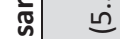

₹

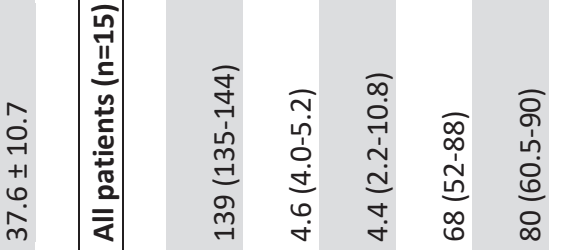

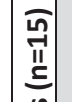

藏 范

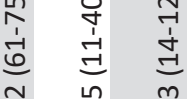

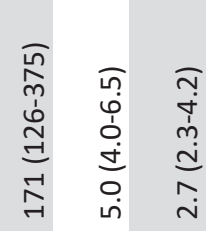

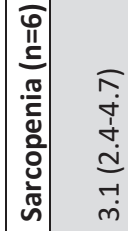



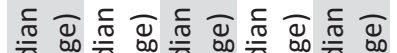

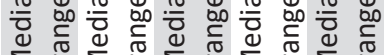

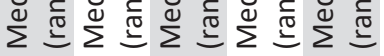

药

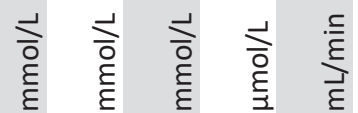

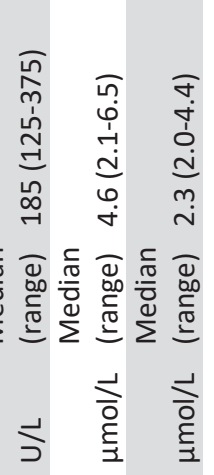

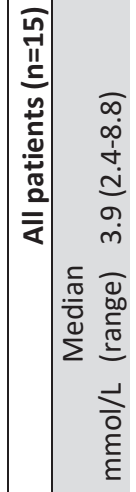

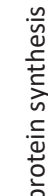

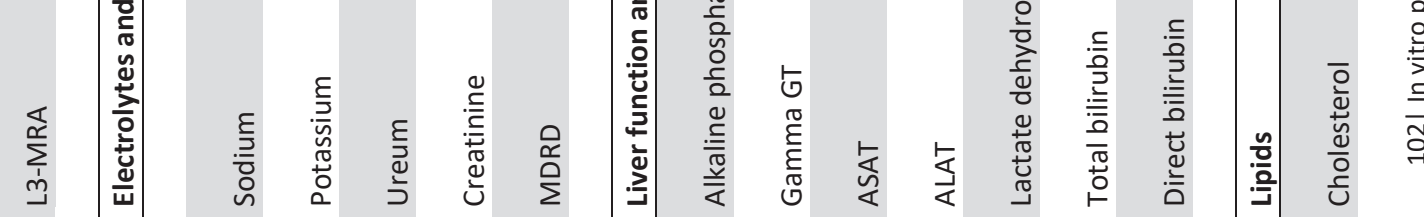




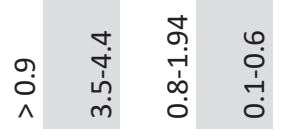

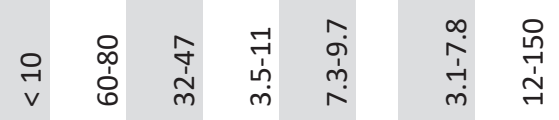

悉壱

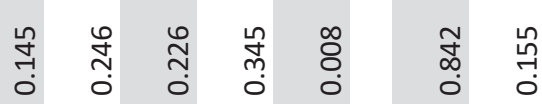

นึก

m

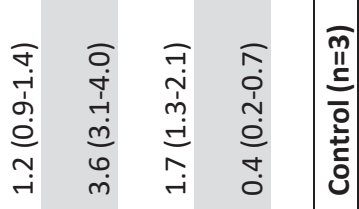

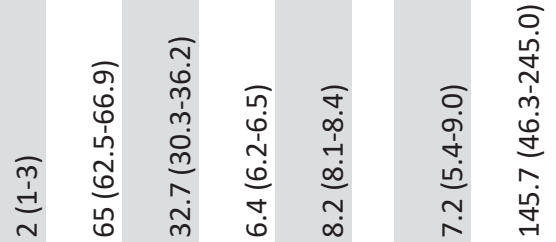

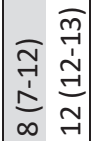

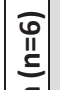

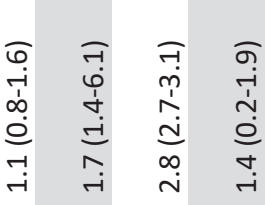

$\frac{\sigma}{\tilde{c}}$

- $\bar{\infty} \overline{0} \overline{0}$

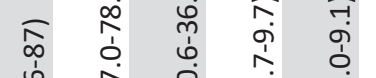

过

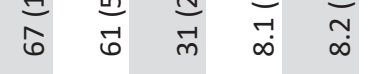

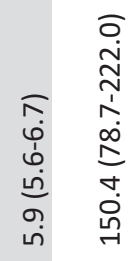

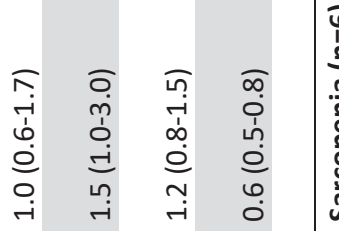

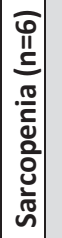

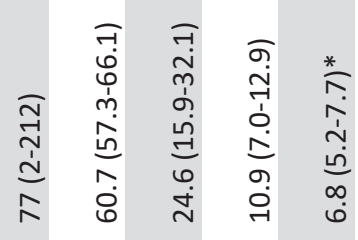

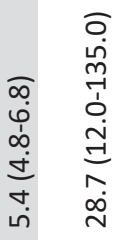

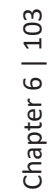

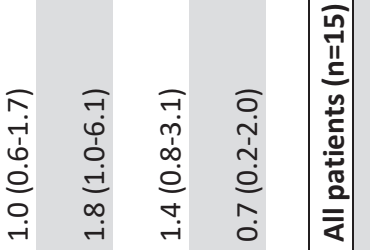

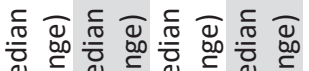

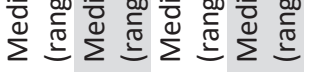

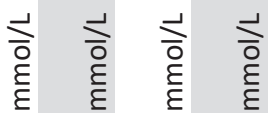

ब. $\overline{6}$

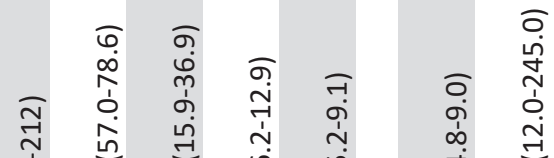

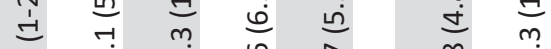

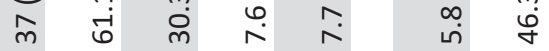

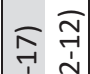

$\dot{m} \underset{ }{ت}$

$\stackrel{m}{\rightarrow} \underset{7}{-1}$

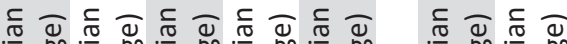
元

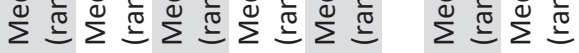

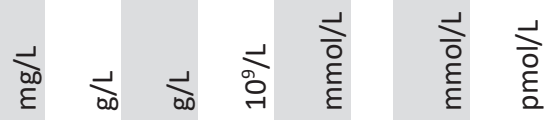




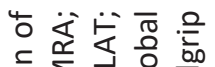

은 学这음 항

गั0

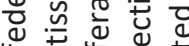

$\frac{4}{\circ}$ ஸे

ปั

흥응요

ᄃ

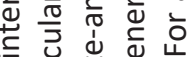

○经营

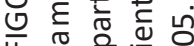

ن.

它安皆

는

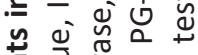

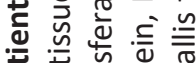

ปั

을흘

† 흘

प $\frac{\pi}{\pi}$

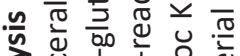

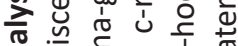

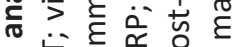

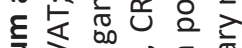

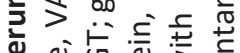

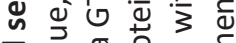

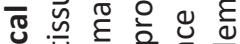

¿

응 旁

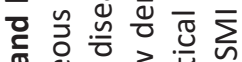

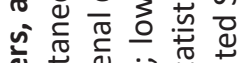

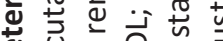

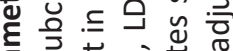

ข

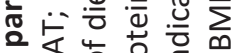

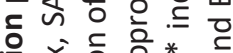

으 х

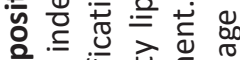

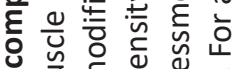

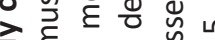

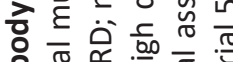

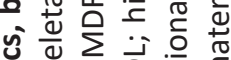

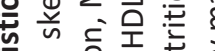

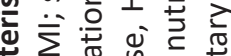

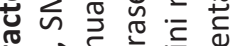

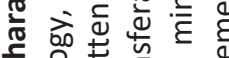

与 음

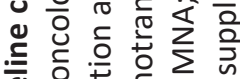

ஸे

ᄀ 응 d

o U્ญ U.

政 
Figure 1 Correlation matrix for Spearman correlations between variables. Positive correlations are shown in blue, inverse correlations in red. Color intensity indicates strength of the correlation. Size of the square indicates level of statistical significance. Mean HU: mean Hounsfield units, Total protein: total serum protein; CRP: c-reactive protein; PG-SGA: patientgenerated subjective global assessment; VAT: visceral adipose tissue, TSA: triceps skinfold assessment, UAC: upper arm circumference, SMI: skeletal muscle index, SAT: subcutaneous adipose tissue, BMI: body mass index, WC: wrist circumference, IMAT: intramuscular adipose tissue, IL-6: interleukin-6, IL-8: interleukin-8, GDF-8: growth differentiation factor 8/myostatin, GDF-15: growth differentiation factor 15, MCP-1: monocyte chemoattractant protein-1.

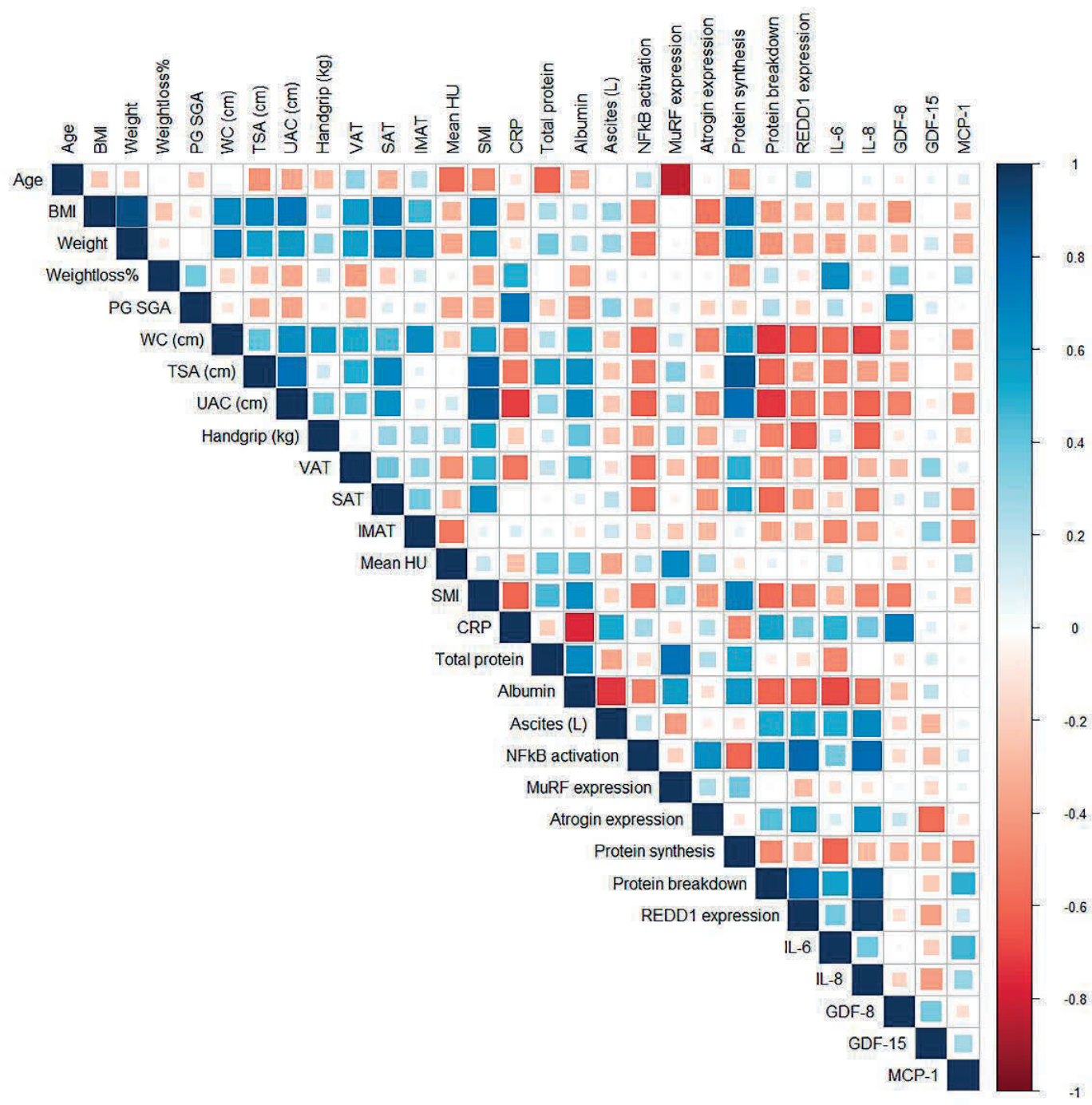




\section{Results}

\section{Cachexia-related patient phenotyping}

The mean SMI of the sarcopenic group was $36.6 \pm 2.9 \mathrm{~cm}^{2} / \mathrm{m}^{2}$, which was significantly lower than the SMI of the non-sarcopenic $(45.2 \pm 2.4 ; p=0.002)$ and benign control $(41.7 \pm 3.6$; $\mathrm{p}=0.05$ ) groups. Although relative weight loss was higher in the sarcopenic vs. the nonsarcopenic vs. the benign control group, the differences were not significant $(p=0.16)$. The median age of the sarcopenic cancer patients was higher than the age of the non-sarcopenic cancer patients ( 68.0 years vs. 59.5 years, $p=0.008$ ). Conversely, mean BMI was lower in the sarcopenic group than in the non-sarcopenic group $\left(22.4 \mathrm{~kg} / \mathrm{m}^{2} \mathrm{vs} .29 .0 \mathrm{~kg} / \mathrm{m}^{2}, \mathrm{p}=0.011\right)$. However, when SMI was adjusted for BMI and age based on figures reported by van der Werf et al. [18], all patients from the present study were above the 10th percentile for low skeletal muscle index in a healthy female population (supplementary material 6).

Mean triceps skinfold $(1.30 \mathrm{~cm})$ was lower in the sarcopenic group than in the non-sarcopenic group (1.98 cm, $\mathrm{p}=0.005)$. CRP levels tended to be higher in the group with a malignancy $(p=0.15)$ and serum albumin was markedly (but not significantly) lower in the sarcopenic group. These and other patient characteristics are listed in table 1.

Figure 1 shows a correlation matrix that illustrates the correlation between body composition parameters and anthropometric measurements across all patients. As expected, SMI was significantly correlated with BMI $\left(r_{s}=0.68, p=0.005\right)$. Furthermore, triceps skinfold $\left(r_{s}=0.83\right.$, $p=0.003)$, upper arm circumference $\left(r_{s}=0.87, p=0.001\right)$, serum albumin $\left(r_{s}=0.64, p=0.01\right)$, and subcutaneous adipose tissue $\left(r_{s}=0.62, p=0.01\right)$, were also all positively correlated with SMI. CRP levels were inversely correlated with $\mathrm{SMI}\left(r_{s}=-0.61, p=0.02\right)$. Patient-reported body weight loss was not associated with any of the other clinical cachexia-related parameters.

\section{Ascites of sarcopenic ovarian cancer patients reduces protein synthesis in C2C12 myotubes We first studied the direct effects of ascites on protein synthesis and protein breakdown using a radioactive isotope tracer method. Interestingly, ${ }^{14} \mathrm{C}$-L-phenylalanine incorporation in $\mathrm{C} 2 \mathrm{C} 12$ myotubes treated with ascites from sarcopenic patients was markedly and significantly lower in comparison to cells treated with non-sarcopenic or benign ascites (3.1 \pm 0.1 vs. $5.5 \pm 0.2$ vs. $5.8 \pm 0.7 \mathrm{nmol} / \mathrm{h} / \mathrm{mg}$ protein, $\mathrm{p}<0.01 \& \mathrm{p}<0.01$, respectively; Figure $2 \mathrm{a}$ ). In contrast, no significant differences in intracellular protein breakdown rates could be detected among the groups, although a trend to higher protein breakdown was observed after exposure of $\mathrm{C} 2 \mathrm{C} 12$ myotubes to ascites from sarcopenic patients (Figure $2 b, p=0.14$ ).}




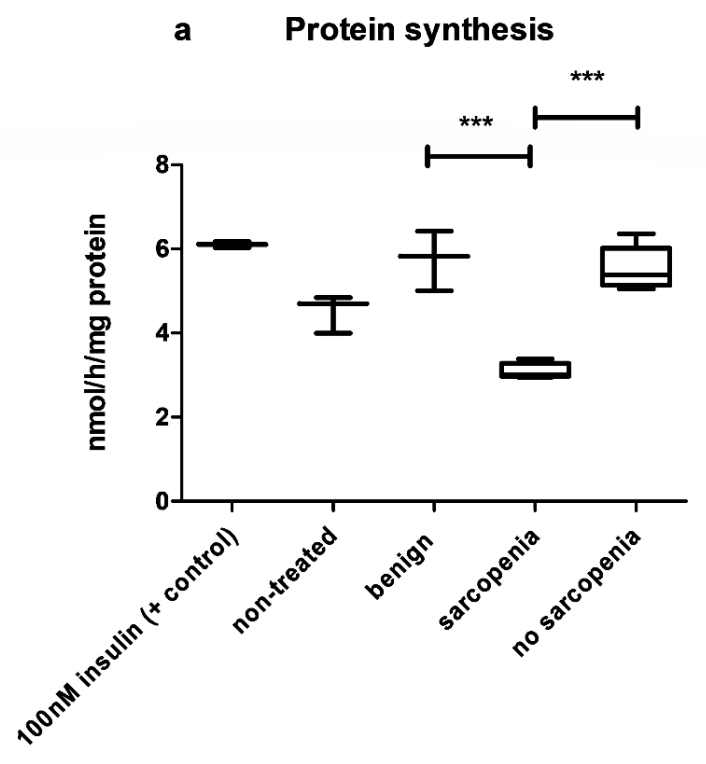

b Protein breakdown

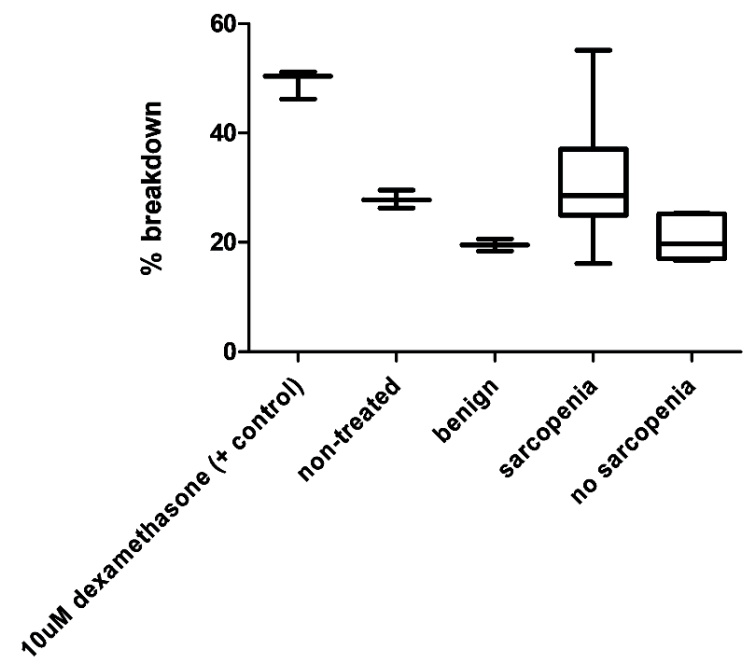


Figure 2 Protein synthesis and protein breakdown in C2C12 myotubes exposed to ascites from ovarian cancer patients. Correction for multiple testing was performed within experimental conditions.

a. Protein synthesis rates. Amino acid incorporation in cells treated with ascites from sarcopenic patients was significantly lower when compared to that in cells treated with ascites from non-sarcopenic cancer patients $(P<0.01)$ or patients with a benign ovarian condition $(p<0.01)$. The positive control was treated with $100 \mathrm{nM}$ insulin, the negative control was $100 \%$ regular differentiation medium. $* * *=\mathrm{P}<0.001$

b. Protein breakdown. No significant differences in proteolytic rates were detected between the groups. The positive control was treated with $10 \mu \mathrm{M}$ dexamethasone, the negative control was $100 \%$ regular differentiation medium.

\section{Concentrations of cachexia-associated factors in ascites of sarcopenic and non-sarcopenic ovarian cancer patients}

We next analyzed ascites for the concentrations of key factors previously implicated in the regulation of protein imbalances in cachexia-associated sarcopenia (Figure 3a-e). There was a trend towards higher median concentrations of IL- 6 and IL- 8 in ascites from sarcopenic patients versus non-sarcopenic patients (IL-6: $6.7 \mathrm{ng} / \mathrm{mL}$ vs $2.6 \mathrm{ng} / \mathrm{mL}(\mathrm{p}=0.06)$, respectively; IL-8: $2.0 \mathrm{ng} / \mathrm{mL}$ vs $1.4 \mathrm{ng} / \mathrm{mL}(\mathrm{p}=0.08)$, respectively). Interestingly, IL-6 levels in ascites were inversely correlated to protein synthetic rates in vitro $\left(r_{s}=-0.66, p=0.02\right)$. IL-6 concentrations in ascites were also strongly correlated with serum CRP levels $\left(r_{s}=0.70, p=0.01\right)$ and with body weight loss $\left(r_{s}=0.69, p=0.01\right.$; Figure 1). Median concentrations of GDF-15 (growth differentiation factor-15), GDF-8/myostatin, and Mcp-1 (monocyte chemoattractant protein 1 ) were not different between the groups. Concentrations of tumor necrosis factor (TNF)- $\alpha$, IL-1 $\beta$, and leukemia inhibitory factor were only detectable in four different samples. Hence no statistical analysis was performed. The total volume of ascites was not significantly different between sarcopenic patients vs. non-sarcopenic patients vs. benign controls.

\section{Ascites of sarcopenic ovarian cancer patients induces NF-KB activity in C2C12 myotubes}

To test if factors present in ovarian cancer ascites directly induced disturbances associated with cachexia in skeletal muscle cells, we exposed differentiated C2C12 myotubes to ascites from sarcopenic and non-sarcopenic patients with malignant or benign ovarian tumors for $24 \mathrm{~h}$, and analyzed several regulatory factors implicated in skeletal muscle protein metabolism. Whereas treatment with $100 \%$ HBSS as a positive control markedly increased expression of MuRF-1 and Atrogin-1, ascites of neither sarcopenic nor non-sarcopenic patients affected mRNA levels of Atrogin-1 ( $p=0.39$ ) or MuRF-1 ( $p=0.80)$ (Figure 4a-b). Likewise, expression of REDD-1 was not significantly affected by ascites from sarcopenic and non-sarcopenic ovarian cancer patients or from benign controls ( $p=0.07$, Figure $4 c)$.

In contrast, NF-KB activity was higher in cells treated with ascites from sarcopenic patients (fold change $2.2 \pm 0.4$ ) than in cells treated with ascites from non-sarcopenic patients (1.2 \pm $0.2, p=0.01)$ or from benign controls $(1.0 \pm 0.1, p=0.04)$ (Figure $4 d$ ). Interestingly, in vitro protein synthesis and breakdown rates across the groups were strongly correlated to NF-KB transcriptional activity $\left(r_{s}=-0.60, p=0.03\right.$ and $r_{s}=0.67, p=0.01$, respectively). NF-kB activity across all groups was further strongly correlated to Atrogin-1 $\left(r_{s}=0.63, p=0.02\right)$ and REDD-1 expression $\left(r_{s}=0.82, p=0.0003\right)$ as well as to IL-8 concentrations in ascites $\left(r_{s}=0.62, p=0.04\right)$. 
a

Interleukin-6

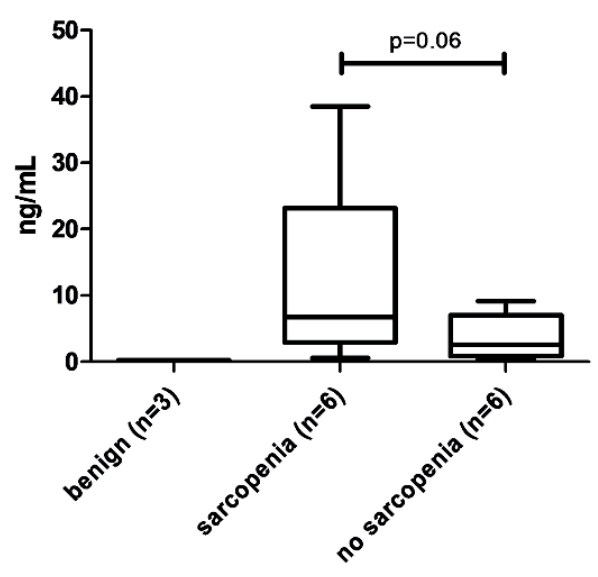

c GDF-8

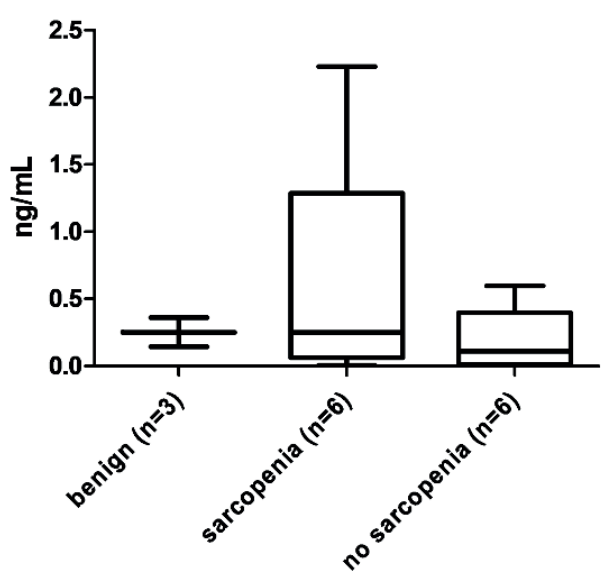

e MCP-1

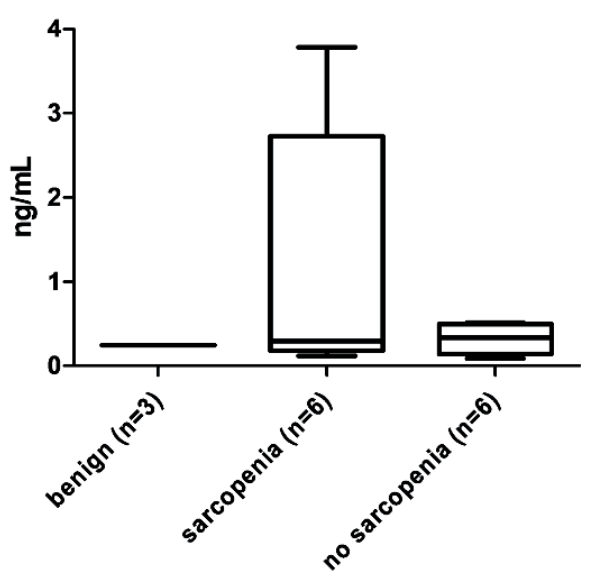

b Interleukin-8
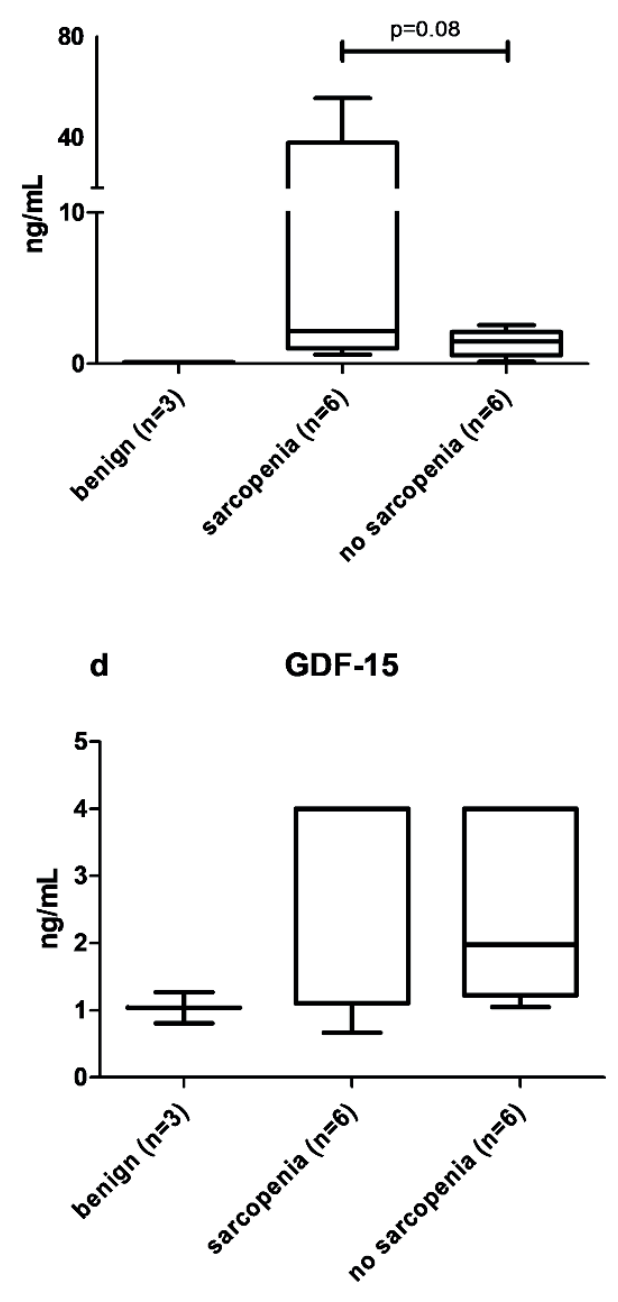
Figure 3 Concentrations of cachexia-inducing factors in ascites of sarcopenic and nonsarcopenic ovarian cancer patients and non-sarcopenic controls with a benign ovarian condition. The box and whiskers graphs display medians with min to max ranges. Also see supplementary material 4.

a. IL-6 concentration in ascites.

b. IL-8 concentration in ascites.

c. Myostatin (GDF-8) concentration in ascites.

d. GDF-15 (growth differentiation factor 15) concentration in ascites.

e. MCP-1 concentration in ascites.

\section{In vitro protein metabolism data correlate with cachexia-related patient phenotypes}

The simultaneous collection of ascites of patients and thorough phenotyping of their cachexiarelated parameters allowed us to assess potential associations between the in vitro effects of ascites on skeletal myotubes and individual patient data. Importantly, a substantial number of sarcopenia related patient measures were correlated to the in vitro protein synthesis and protein breakdown data (Figure 1). For example, C2C12 protein synthesis after ascites exposure was strongly correlated to triceps skinfold $\left(r_{s}=0.88, p=0.02\right)$ and upper arm circumference $\left(r_{s}=0.80, p=0.001\right)$. Conversely, in vitro protein breakdown was strongly inversely correlated to triceps skinfold $\left(r_{s}=-0.60, p=0.04\right)$ and upper arm circumference $\left(r_{s}=-\right.$ $0.73, p=0.007)$. Moreover, in vitro protein synthesis rates of ascites treated $\mathrm{C} 2 \mathrm{C} 12$ myotubes strongly correlated with the SMI of the patients $\left(r_{s}=0.70, p=0.005\right)$. In line, in vitro protein breakdown rates of ascites-stimulated C2C12 myotubes $\left(r_{s}=-0.57, p=0.04\right)$ were inversely correlated with their corresponding patient SMI. Thus, ascites from ovarian cancer patients alters protein turnover of skeletal myotubes in correspondence with the degree of sarcopenia of the patient providing the ascites. 
a

Atrogin-1

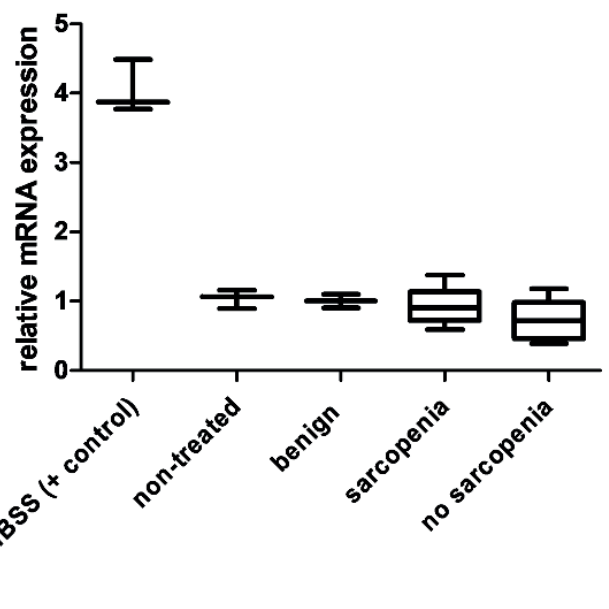

c

REDD1

흥

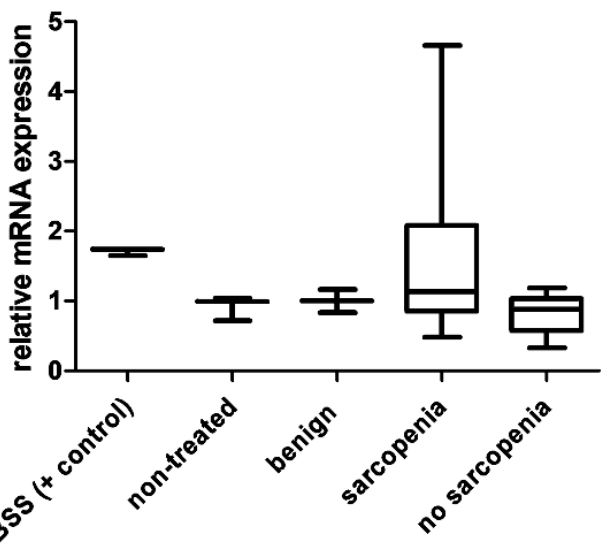

b MuRF1

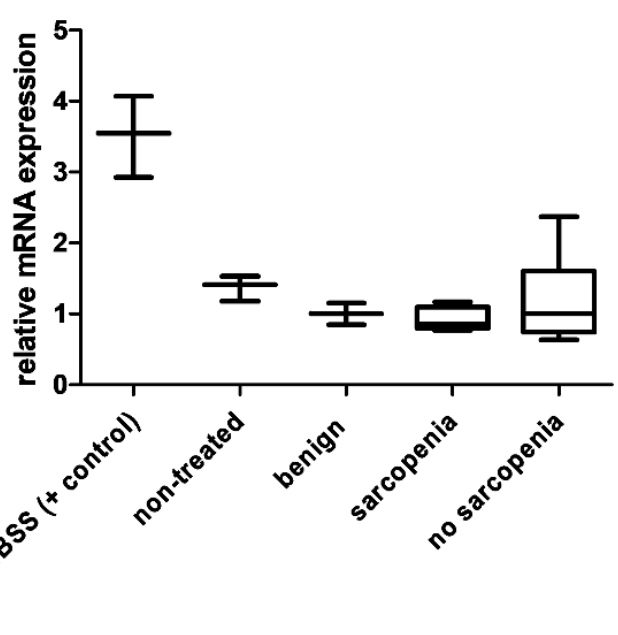

d

NFkB

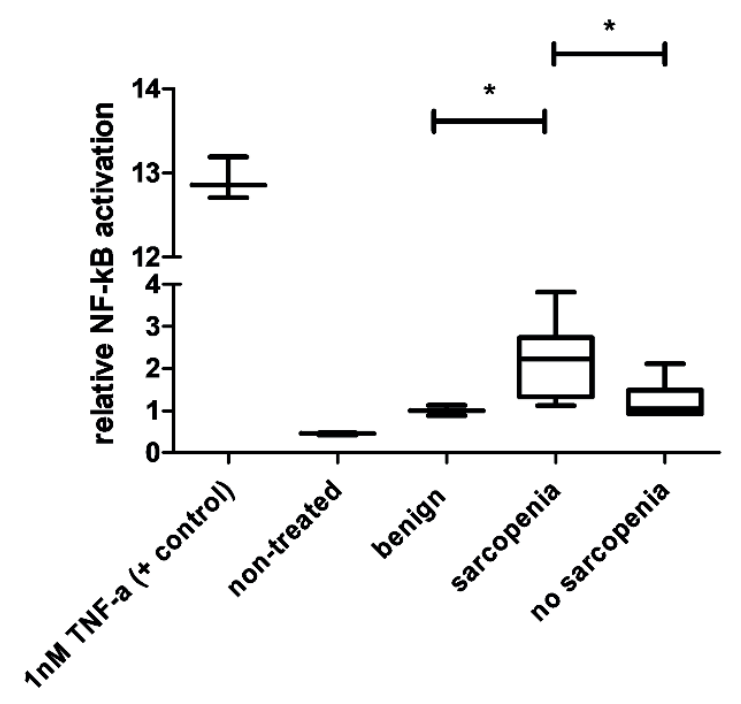


Figure 4 Expression of atrophy related genes and NF-KB activity in $\mathrm{C2C12}$ myotubes exposed to ovarian cancer ascites. Correction for multiple testing was performed within experimental conditions.

a. Relative mRNA expression levels of Atrogin-1 expressed as fold change of the nonsarcopenic benign control group. No differences in Atrogin-1 expression were detected between the groups.

b. Relative mRNA expression levels of MuRF-1 expressed as fold change of the nonsarcopenic benign control group. No differences in MuRF-a expression were detected between the groups.

c. Relative mRNA expression levels of REDD-1 expressed as fold change of the nonsarcopenic benign control group. No differences in REDD-1 mRNA expression were detected between the groups.

d. Transcriptional activity of NF-KB expressed as fold change of the non-sarcopenic benign control group. NF-KB transcriptional activity was significantly increased in cells treated with ascites from sarcopenic patients when compared to cells treated with non-sarcopenic $(p=0.01)$ or benign ascites $(\mathrm{p}=0.04) .{ }^{*}=\mathrm{P}<0.05$. 


\section{Discussion}

This study was performed to assess whether factors present in ascites of sarcopenic versus non-sarcopenic ovarian cancer patients differentially affect protein metabolism in skeletal muscle cells, and to determine if these effects are correlated to cachexia-related patient characteristics. Fifteen patients with a suspected malignancy of the ovary were included and underwent extensive physical screening; their ascites was used for in vitro exposure experiments. Ascites from sarcopenic cancer patients induced NF-KB activity and decreased protein synthesis in skeletal muscle cells. This was not observed with ascites collected from non-sarcopenic cancer patients or from patients with a benign ovarian condition. Interestingly, several strong correlations between the in vitro effects of ascites factors and patient's body composition as well as anthropometric measurements were observed, indicating that ascites represents a novel tool to investigate pathophysiologically relevant aspects of cachexia in vitro.

It is well established that sarcopenia is associated with adverse outcomes in ovarian cancer patients.[1, 19-23] As such, it is of major importance to identify the drivers behind muscle wasting in these patients. The application of ascites to skeletal muscle cells followed by monitoring of its effects on protein metabolism represents a first step towards the identification of factors inducing skeletal muscle protein metabolism aberrations in patients with ovarian cancer. Of note, tumor cell-derived conditioned medium has previously been used in similar in vitro models of muscle wasting.[24, 25] In some of these studies, catabolic responses like myotube atrophy, upregulation of Atrogin-1, NF-kB phosphorylation, and p38MAPK activation were observed after exposure of $\mathrm{C} 2 \mathrm{C} 12$ cells to tumor-derived conditioned medium.[24, 26-28] Several advantages of using ascites over tumor cellconditioned medium can be identified. First of all, ascites is a unique reservoir of highly concentrated tumor-derived compounds as well as systemic factors produced by metabolic tissues in response to tumor factors. Thus, combining ascites and tumor cell or organoidconditioned medium approaches provides unique opportunities to distinguish between direct tumor-derived effects and indirect effects of metabolic tissue alterations caused by tumorfactors from the same patient. Secondly, ascites is relatively easily accessible and available in large quantities. This enables sequential sampling during the course of cancer progression and treatment to study potential progression and treatment-related changes in factors that affect muscle protein metabolism. Thirdly, acquired data can be correlated to clinical parameters when patients are thoroughly phenotyped before collection of ascites. Indeed, we observed several intriguing correlations between patient's body composition and in vitro ascites effects (low SMI and upper arm circumference correlated to low protein synthetic rates, NF-kB activation, and REDD-1 expression) in the current study, suggesting that they are functionally relevant in vivo.

Importantly, factors specifically present in the ascites of sarcopenic patients inhibited protein synthesis and tended to increase protein breakdown in mature myotubes, contributing to a negative protein balance that has been previously shown to be characteristic of muscle wasting in cancer cachexia.[29] Previous mechanistic studies have suggested that the ubiquitin dependent proteasome pathway underlies protein breakdown in skeletal muscle wasting. [6] Two muscle specific ubiquitin ligases, MuRF-1 and Atrogin-1, have been shown to 
be increased in skeletal muscle under atrophic conditions in several disease models and to contribute to protein degradation.[30, 31] However, MuRF-1 and Atrogin-1 expression were not affected by ascites in our experiments, despite a trend to increased protein breakdown induced by ascites from sarcopenic patients. This is in line with a recent report on a cohort of lung cancer patients where MuRF-1 and Atrogin-1 did not appear to be involved in skeletal muscle wasting, and with our own recent data using pancreatic tumor organoids. [32, 33] Although gene expression profiles for atrophy related genes have been established in experimental models of cancer cachexia, they are not a direct or functional measure of skeletal muscle protein turnover. We therefore focused on assessing actual skeletal muscle protein synthesis and breakdown using radioactive isotope tracing with ${ }^{14} \mathrm{C}$-L-phenylalanine, thereby extending the relevance of our findings. Our data show that ascites from patients with muscle loss has more impact on skeletal muscle protein synthesis than on protein breakdown, suggesting that future studies in the field should not only focus on proteolysis-inducing pathways but also investigate protein synthesis pathways.

In this context, the mammalian target of rapamycin complex 1 (mTORC1) is known to promote protein synthesis.[34] In response to environmental factors like nutrient availability, hypoxia, and energy stress, mTORC1 function is altered. REDD-1 expression is considered to be an inhibitor of skeletal muscle protein synthesis by affecting mTORC1.[35] In our experiments, REDD-1 expression was not affected by ascites. However, strong correlations between REDD1 levels and in vitro protein breakdown, NF-KB transcriptional activity, and inverse correlations to handgrip strength, serum albumin, and upper arm circumference were detected.

NF-KB is involved in the transcription of numerous genes encoding cytokines, chemokines, as well as growth regulatory and survival genes. In the context of skeletal muscle atrophy, it has been shown that tumor necrosis factor alpha (TNF- $\alpha$ )-induced NF-KB activation prevents myogenic differentiation.[8] Consequently, it would be interesting to explore the effect of ascites from sarcopenic patients on myoblast differentiation. In the current study of differentiated myotubes, ascites derived from sarcopenic patients increased NF-KB transcriptional activity. Moreover, several interesting correlations between NF-KB transcriptional activity and other protein metabolism related factors including REDD-1 expression, Atrogin-1 expression, and protein breakdown as well as protein synthesis were observed, in line with a prominent role for NF-kB in ascites-induced skeletal muscle metabolism aberrations.

\section{Conclusion}

Collectively, our data show that factors present in ovarian cancer ascites negatively affect protein balance in skeletal muscle cells in vitro, closely reflecting sarcopenia- and cachexiarelated characteristics of the patient providing the ascites. This indicates that the in vitro use of ovarian cancer ascites has high potential to dissect direct and indirect tumor-induced skeletal muscle wasting. Consequently, efforts should be made to identify the compounds responsible for skeletal muscle protein metabolism aberrations induced by ascites, e.g. using proteomics and genomics approaches. This will ultimately help to identify novel drivers of cachexia in ovarian cancer patients and aid in the development of treatment strategies for cancer-induced skeletal muscle wasting. 


\section{Supplementary material}

Supplementary material 1 Primer sequences for the genes of interest.

\begin{tabular}{ll}
\hline Primer & Sequence $\mathbf{( 5}^{\prime}$ - $\mathbf{3}^{\prime} \mathbf{)}$ \\
\hline 62 microglobulin forward & CTTTCTGGTGCTTGTCTCACTGA \\
62 microglobulin reverse & GTATGTTCGGCTTCCCATTCTC \\
Cyclophilin forward & TTCCTCCTTTCACAGAATTATTCCA \\
Cyclophilin reverse & CCGCCAGTGCCATTATGG \\
MuRF1 forward & AAACTTGTGGAGACCGCCAT \\
MuRF1 reverse & GGAGGCTTCTACAATGCTCTTG \\
Atrogin1 forward & AGGAGAATCTGTTCAGCAGCC \\
Atrogin1 reverse & ATCCATGGCGCTCCTTCGTA \\
REDD1 forward & TCGGCGCTTCACTACTGACC \\
REDD1 reverse & CCTAACACCCACCCCATTCC \\
\hline
\end{tabular}

Supplementary material 2 Overview of protein synthesis experiment.

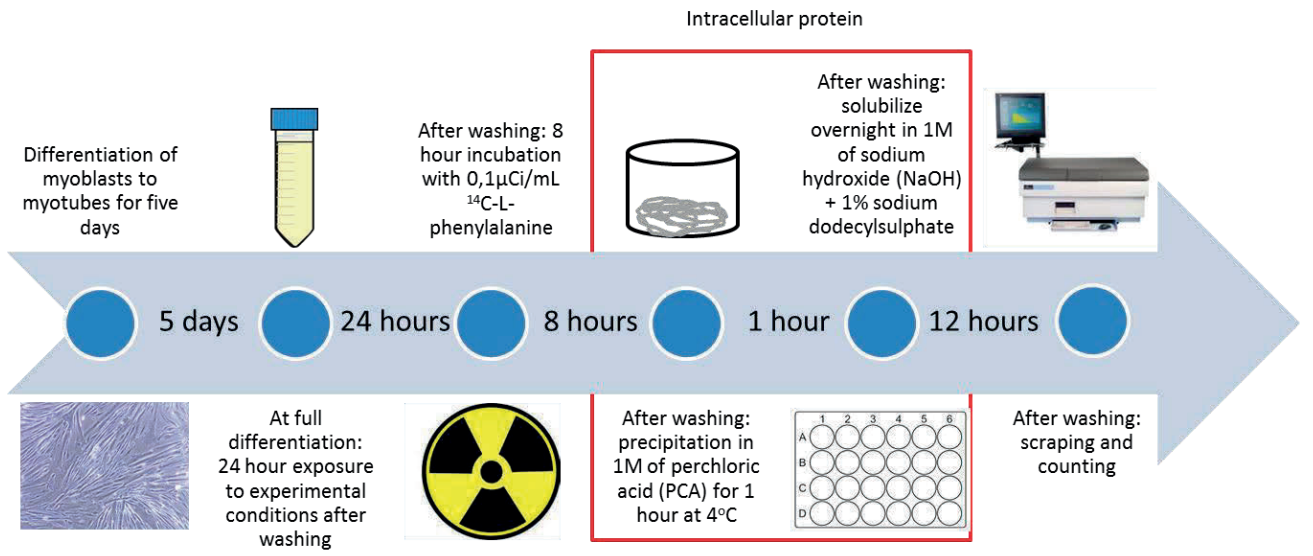


Supplementary material 3 Overview of protein breakdown experiment.

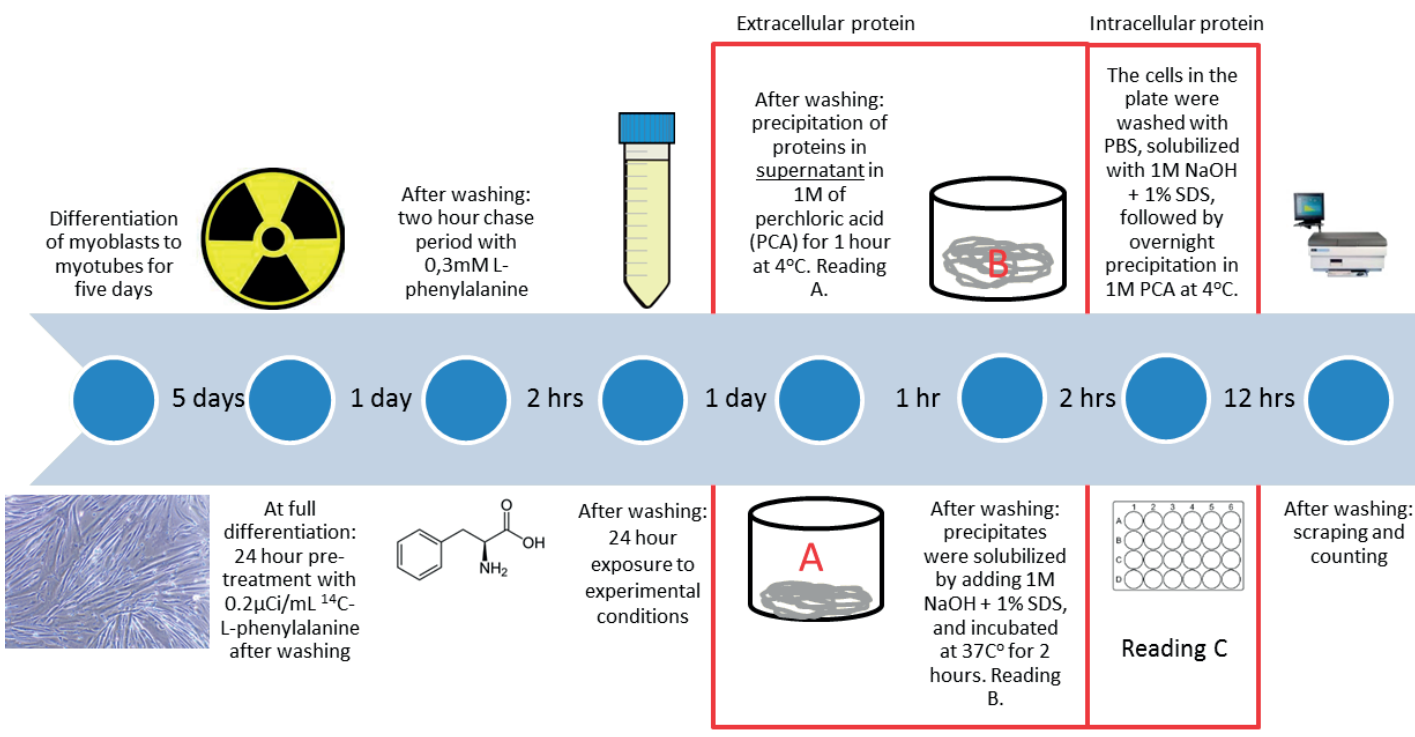

Supplementary material 4 Concentrations of cachexia-associated factors in ascites. GDF 8; growth differentiation factor-8, GDF-15; growth differentiation factor-15, MCP-1; monocyte chemoattractant protein 1, ng/mL; nanogram/milliliter. Medians with ranges are presented. The Kruskall-Wallis test was used to assess statistical significance between all groups.

\begin{tabular}{|c|c|c|c|c|c|c|}
\hline & Unit & $\begin{array}{l}\text { All patients } \\
(n=15)\end{array}$ & Sarcopenia $(n=6)$ & $\begin{array}{l}\text { No } \\
\text { sarcopenia } \\
(n=6)\end{array}$ & Control $(n=3)$ & p-value \\
\hline $\begin{array}{l}\text { Interleukin- } \\
6\end{array}$ & $\begin{array}{l}\mathrm{ng} / \mathrm{mL} \\
\text { (range) }\end{array}$ & 6.5 (1.9-38.5) & $6.7(6.1-38.5)$ & $2.6(0.3-9.1)$ & $0.2(0.2-0.3)$ & 0.06 \\
\hline $\begin{array}{l}\text { Interleukin- } \\
8\end{array}$ & $\begin{array}{l}\mathrm{ng} / \mathrm{mL} \\
\text { (range) }\end{array}$ & $1.4(0-55.5)$ & $2.0(0.6-55.5)$ & $1.4(0.1-2.5)$ & $0(0-0.1)$ & 0.08 \\
\hline GDF-8 & $\begin{array}{l}\mathrm{ng} / \mathrm{mL} \\
\text { (range) }\end{array}$ & $0.2(0-2.2)$ & $0.3(0-2.2)$ & $0.1(0-0.6)$ & $0.2(0.1-0.3)$ & 0.6 \\
\hline GDF-15 & $\begin{array}{l}\mathrm{ng} / \mathrm{mL} \\
\text { (range) }\end{array}$ & $1.8(0.7-4.0)$ & $4.0(0.7-4.0)$ & $1.9(1.1-4.0)$ & $1.0(0.8-1.3)$ & 0.3 \\
\hline МСР-1 & $\begin{array}{l}\mathrm{ng} / \mathrm{mL} \\
\text { (range) }\end{array}$ & $0.3(0-3.8)$ & $0.3(0.1-3.8)$ & $0.4(0-0.5)$ & $0.2(0.2-0.2)$ & 0.3 \\
\hline
\end{tabular}


Supplementary material 5 Age adjusted dynamometer handgrip strength. Differences between groups were not significantly different, $p=0.5 .^{*}$, one missing value. Based on: Dodds RM, Syddall HE, Cooper R, Benzeval M, Deary IJ, Dennison EM, Der G, Gale CR, Inskip HM, Jagger C, Kirkwood TB, Lawlor DA, Robinson SM, Starr JM, Steptoe A, Tilling K, Kuh D, Cooper C, Sayer AA (2014) Grip strength across the life course: normative data from twelve British studies. PLoS One 9:e113637.

\begin{tabular}{|c|c|c|c|c|}
\hline age & sarcopenia $(n=6)$ & no sarcopenia $(n=6)$ & benign $(n=3)$ & \\
\hline $20-29$ & & 1 & & \\
\hline \multicolumn{5}{|l|}{$30-39$} \\
\hline $40-49$ & & 1 & & \\
\hline $50-59$ & & 1 & & \\
\hline $60-69$ & 3 & 3 & 2 & \\
\hline $70-79$ & 3 & & & \\
\hline $80-89$ & & & 1 & \\
\hline \multicolumn{5}{|l|}{ sarcopenia } \\
\hline percentile & $<p 5$ & $<p 10$ & $<p 15$ & $<p 50$ \\
\hline \multicolumn{5}{|l|}{$20-29$} \\
\hline \multicolumn{5}{|l|}{$30-39$} \\
\hline \multicolumn{5}{|l|}{$40-49$} \\
\hline \multicolumn{5}{|l|}{$50-59$} \\
\hline $60-69$ & & 1 & 2 & \\
\hline $70-79$ & & 1 & & 2 \\
\hline \multicolumn{5}{|l|}{$80-89$} \\
\hline overall & & 2 & 2 & 2 \\
\hline \multicolumn{5}{|l|}{ no sarcopenia } \\
\hline percentile & $<p 5$ & $<\mathrm{p} 10$ & $<p 15$ & $<p 50$ \\
\hline $20-29$ & & 1 & & \\
\hline \multicolumn{5}{|l|}{$30-39$} \\
\hline $40-49$ & & & 1 & \\
\hline $50-59$ & 1 & & & \\
\hline $60-69$ & & & 3 & \\
\hline \multicolumn{5}{|l|}{$70-79$} \\
\hline \multicolumn{5}{|l|}{$80-89$} \\
\hline overall & 1 & 1 & 4 & \\
\hline \multicolumn{5}{|l|}{ benign* } \\
\hline percentile & $<p 5$ & $<p 10$ & $<\mathrm{p} 15$ & $<p 50$ \\
\hline \multicolumn{5}{|l|}{$20-29$} \\
\hline \multicolumn{5}{|l|}{$30-39$} \\
\hline \multicolumn{5}{|l|}{$40-49$} \\
\hline \multicolumn{5}{|l|}{$50-59$} \\
\hline $60-69$ & & & 1 & \\
\hline \multicolumn{5}{|l|}{$70-79$} \\
\hline $80-89$ & & & & 1 \\
\hline
\end{tabular}




\begin{tabular}{lcccc}
\hline overall & & & 1 & 1 \\
\hline & $<\mathrm{p} 5$ & $<\mathbf{p} 10$ & $<\mathrm{p}$ 15 & $<\mathrm{p} 50$ \\
\hline sarcopenia & & 2 & 2 & 2 \\
no sarcopenia & 1 & 1 & 4 & \\
benign & & & 1 & 1 \\
\hline $\mathrm{X}^{2}: \mathrm{p}=0.5$ & & &
\end{tabular}

Supplementary material 6 Reference values for age and BMI adjusted values of SMI based on a healthy kidney donor population: van der Werf, A., et al., Percentiles for skeletal muscle index, area and radiation attenuation based on computed tomography imaging in a healthy Caucasian population. Eur J Clin Nutr, 2018. 72(2): p. 288-296. Since BMI was significantly lower in the sarcopenic group whilst age was significantly higher, SMI values were compared against reference percentiles of a healthy cohort of female kidney donors. When adjusted for $\mathrm{BMI}$ and age, SMI values from the current cohort were all above the p5-p10 of the reference cohort. Logistic regression analysis revealed no significant association between $\mathrm{BMI}$, age, and SMI.

\begin{tabular}{|c|c|c|c|}
\hline $\begin{array}{l}\text { Age and BMI adjusted SMI } \\
\text { age }\end{array}$ & $\begin{array}{l}\text { sarcopenia } \\
(n=6)\end{array}$ & $\begin{array}{l}\text { no sarcopenia } \\
(n=6)\end{array}$ & $\begin{array}{l}\text { benign } \\
(n=3)\end{array}$ \\
\hline $20-29$ & & 1 & \\
\hline \multicolumn{4}{|l|}{$30-39$} \\
\hline $40-49$ & & 1 & \\
\hline $50-59$ & & 1 & \\
\hline $60-69$ & 3 & 3 & 2 \\
\hline 70- & 3 & & 1 \\
\hline BMI & $\begin{array}{l}\text { sarcopenia } \\
(n=6)\end{array}$ & $\begin{array}{l}\text { no sarcopenia } \\
(n=6)\end{array}$ & $\begin{array}{l}\text { benign } \\
(n=3)\end{array}$ \\
\hline \multicolumn{4}{|l|}{$17-20$} \\
\hline $20-25$ & 6 & & 1 \\
\hline $25-30$ & & 4 & 2 \\
\hline $30-35$ & & 2 & \\
\hline p5 cut-off adjusted for BMI and age & $\begin{array}{l}<5 \text { th } \\
\text { percentile }\end{array}$ & $>5$ th percentile & \\
\hline sarcopenia & 0 & 6 & \\
\hline no sarcopenia & 0 & 6 & \\
\hline benign & 0 & 3 & \\
\hline p10 cut-off adjusted for BMI and age & $\begin{array}{l}<10 \text { th } \\
\text { percentile }\end{array}$ & $\begin{array}{l}>10 \text { th } \\
\text { percentile }\end{array}$ & \\
\hline sarcopenia & 0 & 6 & \\
\hline no sarcopenia & 0 & 6 & \\
\hline benign & 0 & 3 & \\
\hline
\end{tabular}




\section{References}

1. Ubachs, J., et al., Sarcopenia and ovarian cancer survival: a systematic review and metaanalysis. J Cachexia Sarcopenia Muscle, 2019. 10(6): p. 1165-1174.

2. Pettersen, K., et al., Cancer cachexia associates with a systemic autophagy-inducing activity mimicked by cancer cell-derived IL-6 trans-signaling. Sci Rep, 2017. 7(1): p. 2046.

3. Pin, F., et al., Growth of ovarian cancer xenografts causes loss of muscle and bone mass: a new model for the study of cancer cachexia. J Cachexia Sarcopenia Muscle, 2018. 9(4): p. 685-700.

4. Loumaye, A., et al., Role of Activin A and myostatin in human cancer cachexia. J Clin Endocrinol Metab, 2015. 100(5): p. 2030-8.

5. Kir, S., et al., Tumour-derived PTH-related protein triggers adipose tissue browning and cancer cachexia. Nature, 2014. 513(7516): p. 100-4.

6. Yuan, L., et al., Muscle-specific E3 ubiquitin ligases are involved in muscle atrophy of cancer cachexia: an in vitro and in vivo study. Oncol Rep, 2015. 33(5): p. 2261-8.

7. Britto, F.A., et al., REDD1 deletion prevents dexamethasone-induced skeletal muscle atrophy. Am J Physiol Endocrinol Metab, 2014. 307(11): p. E983-93.

8. Langen, R.C., et al., Inflammatory cytokines inhibit myogenic differentiation through activation of nuclear factor-kappaB. FASEB J, 2001. 15(7): p. 1169-80.

9. Ahmed, N. and K.L. Stenvers, Getting to know ovarian cancer ascites: opportunities for targeted therapy-based translational research. Front Oncol, 2013. 3: p. 256.

10. Adam, R.A. and Y.G. Adam, Malignant ascites: past, present, and future. J Am Coll Surg, 2004. 198(6): p. 999-1011.

11. Kipps, E., D.S. Tan, and S.B. Kaye, Meeting the challenge of ascites in ovarian cancer: new avenues for therapy and research. Nat Rev Cancer, 2013. 13(4): p. 273-82.

12. Laky, B., et al., Malnutrition among gynaecological cancer patients. Eur J Clin Nutr, 2007. 61(5): p. 642-6.

13. Tan, D.S., R. Agarwal, and S.B. Kaye, Mechanisms of transcoelomic metastasis in ovarian cancer. Lancet Oncol, 2006. 7(11): p. 925-34.

14. Matte, I., et al., Profiling of cytokines in human epithelial ovarian cancer ascites. Am J Cancer Res, 2012. 2(5): p. 566-80.

15. Talbert, E.E., et al., Circulating monocyte chemoattractant protein-1 (MCP-1) is associated with cachexia in treatment-naive pancreatic cancer patients. J Cachexia Sarcopenia Muscle, 2018. 9(2): p. 358-368.

16. Penson, R.T., et al., Cytokines IL-1beta, IL-2, IL-6, IL-8, MCP-1, GM-CSF and TNFalpha in patients with epithelial ovarian cancer and their relationship to treatment with paclitaxel. Int J Gynecol Cancer, 2000. 10(1): p. 33-41.

17. Yaffe, D. and O. Saxel, Serial passaging and differentiation of myogenic cells isolated from dystrophic mouse muscle. Nature, 1977. 270(5639): p. 725-7.

18. van der Werf, A., et al., Percentiles for skeletal muscle index, area and radiation attenuation based on computed tomography imaging in a healthy Caucasian population. Eur J Clin Nutr, 2018. 72(2): p. 288-296.

19. Rutten, I.J., et al., The influence of sarcopenia on survival and surgical complications in ovarian cancer patients undergoing primary debulking surgery. Eur J Surg Oncol, 2017. 43(4): p. 717724.

20. Rutten, I.J., et al., Loss of skeletal muscle during neoadjuvant chemotherapy is related to decreased survival in ovarian cancer patients. J Cachexia Sarcopenia Muscle, 2016. 7(4): p. 45866.

21. Aust, S., et al., Skeletal Muscle Depletion and Markers for Cancer Cachexia Are Strong Prognostic Factors in Epithelial Ovarian Cancer. PLoS One, 2015. 10(10): p. e0140403.

22. Bronger, H., et al., Sarcopenia in Advanced Serous Ovarian Cancer. Int J Gynecol Cancer, 2017. 27(2): p. 223-232. 
23. Kumar, A., et al., Muscle composition measured by CT scan is a measurable predictor of overall survival in advanced ovarian cancer. Gynecol Oncol, 2016. 142(2): p. 311-6.

24. Yang, J., et al., ZIP4 Promotes Muscle Wasting and Cachexia in Mice With Orthotopic Pancreatic Tumors by Stimulating RAB27B-Regulated Release of Extracellular Vesicles From Cancer Cells. Gastroenterology, 2019. 156(3): p. 722-734 e6.

25. Guigni, B.A., et al., Effects of conditioned media from murine lung cancer cells and human tumor cells on cultured myotubes. Am J Physiol Endocrinol Metab, 2020. 318(1): p. E22-E32.

26. Gao, S. and J.A. Carson, Lewis lung carcinoma regulation of mechanical stretch-induced protein synthesis in cultured myotubes. Am J Physiol Cell Physiol, 2016. 310(1): p. C66-79.

27. Stitt, T.N., et al., The IGF-1/PI3K/Akt pathway prevents expression of muscle atrophy-induced ubiquitin ligases by inhibiting FOXO transcription factors. Mol Cell, 2004. 14(3): p. 395-403.

28. Sandri, M., et al., Foxo transcription factors induce the atrophy-related ubiquitin ligase atrogin1 and cause skeletal muscle atrophy. Cell, 2004. 117(3): p. 399-412.

29. Brown, J.L., et al., Protein imbalance in the development of skeletal muscle wasting in tumourbearing mice. J Cachexia Sarcopenia Muscle, 2018. 9(5): p. 987-1002.

30. Khal, J., et al., Expression of the ubiquitin-proteasome pathway and muscle loss in experimental cancer cachexia. Br J Cancer, 2005. 93(7): p. 774-80.

31. Lazarus, D.D., et al., A new model of cancer cachexia: contribution of the ubiquitin-proteasome pathway. Am J Physiol, 1999. 277(2): p. E332-41.

32. Op den Kamp, C.M., et al., Pre-cachexia in patients with stages I-III non-small cell lung cancer: systemic inflammation and functional impairment without activation of skeletal muscle ubiquitin proteasome system. Lung Cancer, 2012. 76(1): p. 112-7.

33. Vaes, R.D.W., et al., Generation and initial characterization of novel tumour organoid models to study human pancreatic cancer-induced cachexia. J Cachexia Sarcopenia Muscle, 2020. 11(6): p. 1509-1524.

34. Duval, A.P., et al., mTOR and Tumor Cachexia. Int J Mol Sci, 2018. 19(8).

35. Brugarolas, J., et al., Regulation of mTOR function in response to hypoxia by REDD1 and the TSC1/TSC2 tumor suppressor complex. Genes Dev, 2004. 18(23): p. 2893-904.

36. von Haehling S, Morley JE, Coats AJ, Anker SD. Ethical guidelines for publishing in the journal of cachexia, sarcopenia and muscle: update 2017. J Cachexia Sarcopenia Muscle 2017;8:10811. 
Chapter 6 | 121 


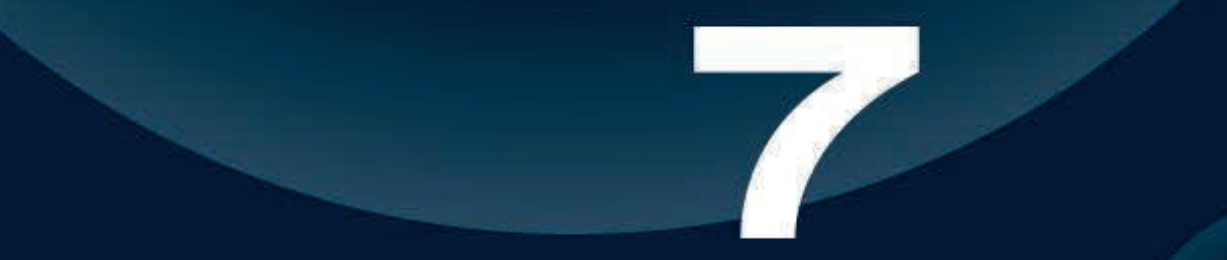


General Discussion 


\section{General Discussion}

The main objective of this thesis was to study cancer cachexia in patients with advanced stage ovarian cancer. Cachexia is the loss of body weight ( $>5 \%$ over the past six months) with or without loss of muscle mass. Diagnosing cachexia in ovarian cancer patients can be challenging since weight loss is often masked by accumulation of ascites. Therefore, it is more reliable to quantify skeletal muscle quantity and/or quality instead of weight loss in these patients. Since abdominal CT scans are routinely used for diagnosis, treatment evaluations, and follow up of ovarian cancer, they have become the most frequently used method for body composition assessment. The total skeletal muscle area at the third lumbar level corrected for stature, the so-called Skeletal Muscle Index (SMI), as assessed by CT scan analysis, correlates reasonably well with total lean body mass.[1]

In Chapter 2, all available literature on body composition measurements related to outcome in ovarian cancer patients was reviewed in a meta-analysis. The goal was to assess the existing body of evidence concerning the cut-offs used to define sarcopenia and to ascertain the association between sarcopenia and survival. Only ten studies could be included for a qualitative synthesis.[2-11] All studies were retrospective of nature and, therefore, were considered to have a high risk of bias. Nine studies used CT-scans to assess skeletal muscle volume and one used a bio-impedance analysis.[11] Predefined and previously published cutoffs were frequently used for determining sarcopenia with a SMI that ranged from 38.5 $\mathrm{cm}^{2} / \mathrm{m}^{2}$ to $41.5 \mathrm{~cm}^{2} / \mathrm{m}^{2}$. One study used multiple cut-offs for defining sarcopenia and performed multiple analyses on the relationship between skeletal muscle attenuation and ovarian cancer outcome.[10] Cohorts were very heterogeneous, i.e. disease stage between included studies ranged from FIGO I-IV and performance indexes were rarely ever mentioned. Due to the aforementioned shortcomings, imprecision and indirectness were detected in the forest plots. Indirectness was defined by the correspondence of the population of interest to the population included for analysis. Imprecision was defined by insufficient sample size and very wide confidence intervals, crossing the null in $\geq 50 \%$ cases. Although sarcopenia was related to outcome in ovarian cancer patients, it was very difficult to draw conclusions from the meta-analysis. As a consequence, it was impossible to discern a single cut-off value for a single point in time CT scan from the literature.

A more unbiased approach to determine the cut-off for sarcopenia is to use standard deviations, tertiles, or quartiles, or to use optimum stratification techniques instead of predefined cut-offs. Optimum stratification methods are based on log-rank statistics. They solve the threshold value of a continuous covariate (SMI) that best separates patients with and without sarcopenia with respect to time to an event outcome.[17] By using these methods instead of using cut-offs which were previously published, cut-offs are most applicable to the presented cohort. An optimum stratification technique was used in chapter 4 in which the relationship between sarcopenia and survival as well as surgical complications in ovarian cancer patients undergoing primary debulking surgery was examined. The optimal cut-off was ultimately determined at a SMI of $38.73 \mathrm{~cm}^{2} / \mathrm{m}^{2}$ but a range between a SMI of $37.90 \mathrm{~cm}^{2} / \mathrm{m}^{2}$ and $40.25 \mathrm{~cm}^{2} / \mathrm{m}^{2}$ would also have yielded significant results in univariate Kaplan-Meier analysis. Any patient with a SMI below the cut-off as determined by optimum stratification was classified as sarcopenic. In this cohort, baseline scans of ovarian cancer patients scheduled for primary debulking surgery were analyzed for body composition. Although sarcopenia was 
associated with worse outcome in the Kaplan-Meier estimate, the result was lost in multivariate cox regression. Completeness of debulking surgery, surgery in a specialized center, and development of a Clavien-Dindo grade $\geq 3$ complications were stronger predictors for overall survival. Low skeletal muscle radiation attenuation (SMRA) was associated with the development of surgical complications, although the effect was lost again in multivariate analysis. Interobserver agreement for all body composition measurements in this study were excellent ( $r_{s}$ 0.969-0.997). Although optimum stratification techniques may represent less biased approaches to determine a cut-off for specific cohorts, they have the major drawback that they cannot be used to compare study results because they are cohort-specific.

Since assessment of total skeletal muscle area at the third lumbar level of abdominal CT scans can be time consuming, several studies suggested the measurement of the psoas muscle area as a surrogate marker. Evidently, measuring only one muscle group is less time-consuming. The association between psoas muscle area and outcome has been examined in cohorts of urothelial, pancreatic, and biliary tract malignancies.[12-14] Longitudinal changes in total skeletal muscle area, interobserver agreement, and correlation with psoas muscle area were assessed in ovarian cancer patients in chapter 3 . Whereas total skeletal muscle area is known to correlate well with total body lean body mass [11], there is no evidence that the same is true for psoas muscle area, nor any other skeletal muscle group at the third lumbar level. Although interobserver agreement was good for all methods of quantification, correlations and kappa agreement between the different methods of quantification were poor. This finding was reproduced in chapter 4 in which psoas muscle index also poorly correlated with SMI. Additionally, only total skeletal muscle area was associated with overall survival. Besides being not representative of total skeletal muscle area, the psoas muscle can also be specifically affected by degenerative lumbar spine conditions which can cause local atrophy, therefore making it unsuitable for these assessments.[15] Since psoas muscle area represents only a small portion of total skeletal muscle area at the third lumbar level, it might also not be sensitive enough for detecting changes.[16] Therefore, measurement of skeletal muscle should be done with total skeletal muscle area instead of psoas area. Other approaches, such as quantification of skeletal muscle quality, can also be used. An example of this involves assessing the degree of myosteatosis, which is reflected by skeletal muscle attenuation, or mean Hounsfield units of the skeletal muscle area on CT-scans. However, when assessing muscle attenuation, one has to realize that measurements are influenced by the contrast phase and the quality of the CT scan.[12,13] For example, a difference of at least $8 \mathrm{HU}$ in mean SMRA has been detected between the venous-portal and arterial phase.[12] Therefore, body composition should only be assessed on the basis of total skeletal muscle area and only in cohorts for which CT-scans with the same contrast phase are available.

\section{Defining sarcopenia by assessing SMI changes over time using CT scan analysis}

A solution for the problem of finding the optimal cut-off for sarcopenia may lie in changing the definition of cancer-induced cachexia and sarcopenia. Instead of determining skeletal muscle mass at a single point in time, it might be more valid to determine changes over time. Longitudinal measurements of body composition will also eliminate the need for establishing uniform cut-offs for defining baseline cachexia or sarcopenia. Using this technique, Rutten et al. showed an association between longitudinal body composition changes and outcome in 
ovarian cancer patients ( $n=123$ ) who underwent three cycles of neo-adjuvant chemotherapy for high stage ovarian cancer.[4] Patients with a loss of SMI had a significant shorter overall survival (OS) compared to patients who maintained or gained skeletal muscle mass (median OS $916 \pm 99$ days vs. $1431 \pm 470$ days, respectively).[4] Inspired by this study, we aimed to reproduce these results in a larger cohort (chapter 5). FIGO stage III ovarian cancer patients included in the OVHIPEC trial [26] were included for body composition analysis. CT-scans were made before and after two cycles of neo-adjuvant chemotherapy, and thresholds to define changes in SMI were the same as in the previous cohort by Rutten et al.[4] In contrast to the study of Rutten et al., alterations in skeletal muscle were not associated with OS or recurrence free survival in this cohort. This may be due to the fact that the OVHIPEC cohort was very homogeneous and had a relatively better prognosis than the cohort used in the study by Rutten et al. In the OVHIPEC cohort, all patients had FIGO stage III disease (FIGO stage IV was excluded), the same performance index, and at least cytoreduction to $<1 \mathrm{~cm}$ at interval debulking surgery. In contrast, the association between a decrease in SMI and outcome as mentioned in the study of Rutten et al. may be attributable to the inclusion of relatively frail patients who did not meet the inclusion criteria for the OVHIPEC trial, but had a poor outcome due to adverse events, dose modifications, or incomplete surgery. The strong relationship between skeletal muscle loss and the reported toxicities supports this theory. Earlier findings indicated that increased toxicity from systemic therapy was related to lower lean body mass, which results in reduced volume of distribution, protein binding, metabolism, and clearance of drugs.[14-17] These results make it presumable that a loss of skeletal muscle mass is not an independent predictor of survival, but instead is a more objective measure of patient's performance status and/or treatment burden, affecting the patient's ability to receive or complete treatment, which in turn can affect outcome.

\section{Drivers of ovarian cancer related cachexia}

Skeletal muscle alterations seem to play a significant role in ovarian cancer outcome. Human studies have consistently established connections between serum elevated inflammatory cytokines, most notably tumor necrosis factor- $\alpha$ (TNF- $\alpha$ ) and interleukin- 6 (IL-6), and skeletal muscle wasting [18-22], although one study suggested increased circulating IL-6 was not associated with wasting but rather advanced disease.[23] Rodent models were also used to study the specific effects of cancer on cachexia. Elevated serum levels of IL- 6 were observed in the presence of cancer and the artificial administration of IL- 6 led to skeletal muscle wasting, fat depletion and death.[24] The most important intracellular pathways induced by IL-6 ligand-receptor binding are the JAK/STAT3, ERK and PI3K/Akt pathways. Another transcription factor that plays an important role is Signal Transducer and Activator of Transcription 3 (STAT3). STAT3 plays a pivotal role in the acute phase response, and has been shown to be upregulated in skeletal muscle of cachectic mice[25] whereas pharmacological inhibition of STAT3 attenuated muscle atrophy.[26] Importantly, efforts to target cytokines in human cancer cachexia have thus far not been successful.[27] Administration of the anti-TNF$\alpha$ monoclonal antibody etanercept in advanced cancer patients did not lead to increases in body weight, quality of life, or survival when compared to a placebo.[28] The addition of the anti-TNF- $\alpha$ monoclonal antibody infliximab to systemic chemotherapy led to negative outcomes in cohorts of non-small cell lung cancer patients and pancreatic cancer patients.[29, 30] 
Experimental in-vitro models using immortalized rodent skeletal muscle cells have been used to study the pathophysiology of sarcopenia.[31] TNF- $\alpha$ signaling increased nuclear transcription factor $-\mathrm{KB}$ (NF-KB) induced transcription of ubiquitin proteasome gene muscle RING finger protein-1 (MuRF1) which codes for E3 ligases specific to myofibrillar proteins. E3 ligases are enzymes which target specific substrate proteins to be broken down by the ubiquitin proteasome, and their increased transcription would therefore lead to increased breakdown of myofibrillar proteins by the ubiquitin proteasome.[32-34] Conversely, inhibition of NF-KB has been shown to alleviate cytokine driven atrophy of muscle.[35] Tumor bearing rodents in a model of breast cancer presented with increased systemic inflammation due to high levels of TNF- $\alpha$ and IL- 6 and they also showed increased gene expression and protein levels of MuRF1 and Atrogin-1 in hind limb muscles.[36] Primary muscle cells from biopsied human or murine samples are also an attractive method for studying cancer cachexia since the cells retain some of their in-vivo properties, more so than immortalized cell lines do.[31] However, gene expression patterns observed in mouse models of cachexia were not reflective of those in muscle biopsies of cachectic human pancreatic cancer patients. [37] Similar results were obtained from a cohort of cachectic lung cancer patients who showed no difference in E3 ubiquitin ligase expression despite reduced muscle mass and function.[38] Conditioned medium approaches have been used to study the effects of tumor-derived compounds on cultured myotubes.[39, 40] Whereas some studies reported myotube atrophy and upregulation of catabolic gene expression markers [41-43], others did not.[40] Therefore, the focus of the exposure experiments which are part of this thesis shifted towards functional measurements of protein incorporation and breakdown. In chapter 6 , we demonstrated that in vitro exposure of skeletal muscle cells to ascites derived from cachectic ovarian cancer patients leads to a marked decrease in protein synthesis. Additionally, no differences in gene expression profiles of MuRF and Atrogin-1 were detected between sarcopenic and nonsarcopenic groups. These findings suggest that the E3 ligases MuRF and Atrogin-1 do not play a major role in ovarian cancer induced skeletal muscle metabolism alterations, as seen before $[37,38,40]$, especially since protein synthesis was altered. Ovarian cancer ascites appears to be a promising model for (conditioned) medium exposure experiments since it is a unique reservoir of soluble compounds that are present in much higher concentrations when compared to human serum.[44]

Based on these findings, it is important to extensively assess cancer patients for physical fitness and use functional measurements of amino acid incorporation in models of cancer cachexia. The extensive physical and nutritional assessment of the included patients revealed several intriguing correlations between clinical and in-vitro outcomes. Additionally, half of the included patients were non-cachectic and thereby provided an excellent control group for distinguishing specific cachexia related outcomes. When performing body composition analyses, clinical cachexia status is often overlooked. Body composition measurements should not be used as the only proxy for outcome in cancer patients. Extensive screening for clinical measurements of cachexia should be performed in all future experimental (human and animal) models to discern specific cancer cachexia related outcomes from cancer related outcomes. 


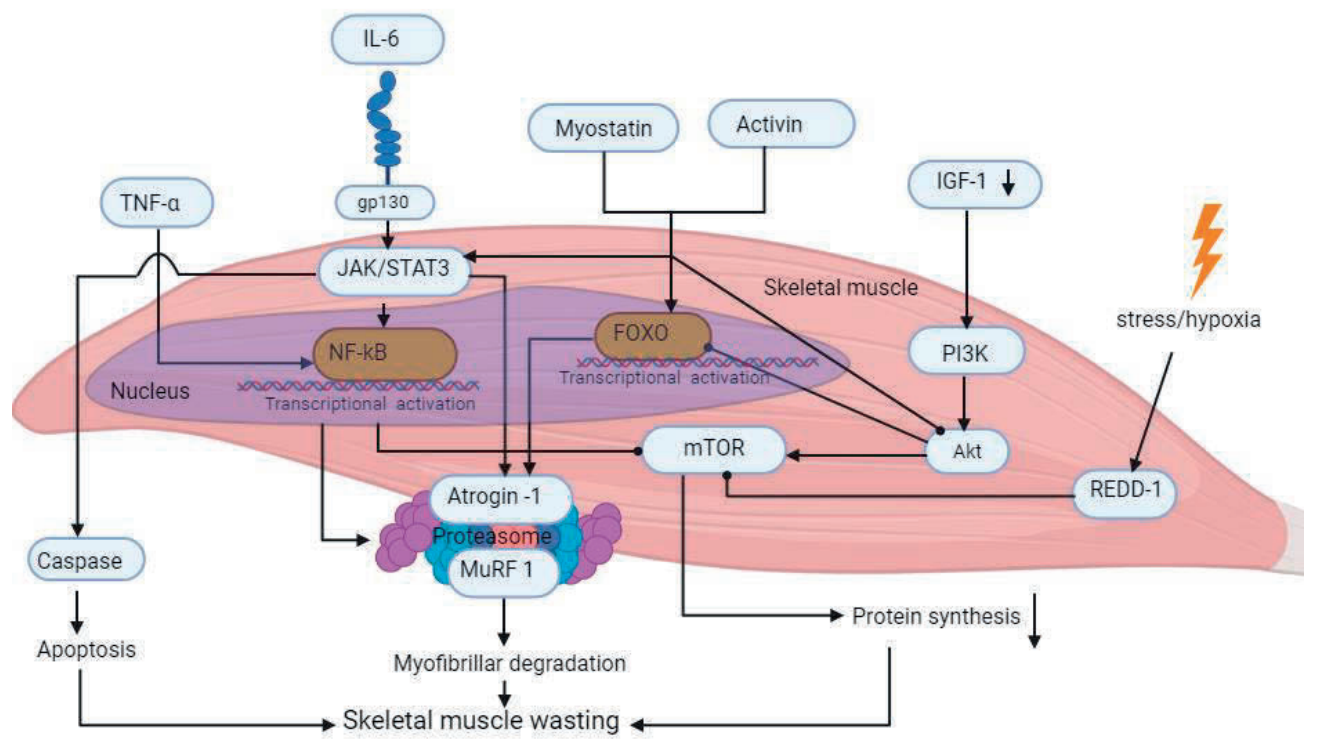

Figure 1 Intracellular signals participating in skeletal muscle wasting. Many intracellular signals are activated by inflammatory mediators (such as cytokines and myostatin). Cytokines activate the NF-kB and FOXO transcription factors to increase transcription of genes encoding for ubiquitin ligases MuRF 1 and Atrogin-1. These ubiquitin ligases are involved in the breakdown of myofibrillar proteins. However, in results published in this thesis, increased transcription of genes encoding for ubiquitin ligases was not observed in cells exposed to ascites derived from sarcopenic patients. Cytokines can also activate the JAK pathways leading to increased apoptosis through increased caspase activity. A decrease in IGF-1 during skeletal muscle wasting suppresses protein synthesis. IGF-1 promotes protein synthesis through AKT and mTOR. Myostatin can decrease protein synthesis by inhibiting AKT, whilst activating protein degradation through FOXOs. Chronic IL-6 exposure can activate the JAK/STAT cascade through the gp130 membrane bound receptor. Activation of the JAK/STAT pathway leads to increased caspase activity and increased transcription of ubiquitin ligases through NF-kB activation. REDD-1 is a stress-induced protein and acts as an inhibitor of mTOR. By controlling mTOR activity, REDD-1 regulates the insulin signaling pathway. TNF- $\alpha$; tumor necrosis factor alpha, PIF; proteolysis-inducing factor, IL-6; interleukin-6, IGF-1; insulin-like growth factor 1, JAK; Janus kinase, STAT; signal transducer and activator of transcription proteins, $\mathrm{PI} 3 \mathrm{~K}$; Phosphoinositide 3-kinase, NF-kB; nuclear factor kappa-B, FOXO; forkhead box protein, Akt; protein kinase $B$, mTOR; mechanistic target of rapamycin, REDD-1; regulated in development and DNA damage responses 1, MuRF 1; muscle RING finger protein 1. Arrows indicate an activation. Lines with a dot indicate suppression. 


\section{Future perspectives}

The exact causality between low lean body mass and ovarian cancer outcomes has not yet been fully established. Decreased treatment efficacy due to adverse body composition might be an explanation for the observed effect of low skeletal muscle mass and worse outcome in ovarian cancer patients. It is plausible that sarcopenia-associated dose limiting toxicities (DLTs) lead to a reduction of treatment cycles and a decrease of treatment dose density which eventually result in decreased treatment efficacy. Several studies have already established associations between toxicities and low lean body mass. [14-16] Dose limiting toxicities were established in cohorts of patients with gastro-intestinal and renal malignancies treated with systemic therapies.[45-47] However, evidence in ovarian cancer patients is scarce. It was demonstrated in chapter 5 that a decrease in skeletal muscle mass during neo-adjuvant chemotherapy was strongly associated with adverse events. Currently, chemotherapy dosing is based on the patient's height and weight, or body surface area (BSA), a formula devised over a century ago.[48] BSA does not take fat mass into consideration which might make up a large and unpredictable part of body weight. Most cytotoxic agents are nonlipophilic and their volume of distribution consists of the fat free mass.[49-51] It was additionally demonstrated by Prado et al. that fat free mass is poorly associated with BSA in obese cancer patients and its variation could account for an up to three-fold variation in effective volume of distribution for administered chemotherapy per unit of BSA.[52] Based on these results, there is a risk of overestimation of the administered dose of cytotoxic agents, especially in patients with high $\mathrm{BMI}$ or low lean body mass. A feasibility study in lung cancer patients has been performed to assess whether carboplatin dose could be calculated based on body composition assessment with DEXA.[53] As a next step, these results should be used in a randomized controlled trial in which ovarian cancer patients are randomized for either traditional dosing of chemotherapy vs. a method which takes body composition into account with the aim of reducing (dose limiting) toxicities without impairing the prognosis of the patient.

To further elucidate the relationship between physical activity, body measurements, and treatment related toxicities, the prospective observational study using 'patient recorded intratreatment measurements' (PRIMs) was designed. The protocol is currently under revision by the medical ethics committee. These PRIMs measure the physical activity and body composition at baseline and during treatment with objective internet-based home measurements. These measurements will be correlated to treatment related toxicity with the aim to more accurately predict DLTs. The gained knowledge might enable us to optimize and individualize therapy. Additionally, objective data regarding body weight fluctuations, physical exercise, and nutritional intake will be collected to gain more insight into the severity and evolution of cancer cachexia during treatment. The data gathered during this study might serve as a blueprint for the suggested randomized controlled trial evaluating traditional BSAbased dosing of chemotherapy vs. dosing based on body composition in ovarian cancer patients. Furthermore, these data can also be used in interventional studies, in which the effect of dietary changes or physical exercises on cachexia, DLTs, and/or survival can be studied. 


\section{Defining cachexia}

The current definition of cachexia, based on retrospective patient reported weight loss, loss of skeletal muscle, and $\mathrm{BMI}$, gives clinicians and researchers a valuable though relatively inaccurate tool to distinguish cachectic patients from non-cachectic patients.[54] Moreover, in ovarian cancer patients, weight loss and change in BMI can be masked by the accumulation of ascites. Objective data such as skeletal muscle mass is taken into consideration for defining cachectic patients from non-cachectic patients but usually only a single measurement is taken. This single measurement does not take other internal and external influencing factors into account. Genetic predisposition, age, gender, diet, and physical exercise all may influence body composition, and a single measurement of low SMI does not always indicate cachexia. For example, in a study with healthy kidney donors, the SMI was found to range from 28.2 $\mathrm{cm}^{2} / \mathrm{m}^{2}$ to $54.8 \mathrm{~cm}^{2} / \mathrm{m}^{2}$ amongst all age, BMI, and gender groups.[55] Similar results were observed in a cohort of 1145 healthy kidney donors of all genders and BMI categories, aged 18-86 years, whose median SMI was $47.4 \mathrm{~cm}^{2} / \mathrm{m}^{2}$ (interquartile range 41.9-54.6).[56] In chapter 4, a SMI of $37.90 \mathrm{~cm}^{2} / \mathrm{m}^{2}$ to $40.25 \mathrm{~cm}^{2} / \mathrm{m}^{2}$ would have yielded significant results in univariate Kaplan-Meier survival analysis. This broad range of SMI would be indicative of an effect caused by an effect modifier like age. When no correction is applied for these effect modifiers in survival analyses, less strict cut-offs could be used. It is advisable to perform body composition measurements and stratify for known effect modifiers like age since skeletal muscle mass diminishes with advancing age.[55] In line, stratification for effect modifiers like age has long been performed in anthropometric measurements such as grip strength, which is also majorly influenced by advancing age.[57] However, the 'best' clinical variable that distinguishes cachectic from non-cachectic individuals is a change in skeletal muscle mass over time. This eliminates the age related, genetic, and environmental influence on baseline skeletal muscle measurements, and gives a better insight into body composition alterations through treatment and/or disease progression. I would suggest to define new criteria for cancer induced cachexia and sarcopenia based on longitudinal clinical and radiological data, similar to the RECIST[58] criteria which are used to define tumor response to treatment. Furthermore, standardization of body composition measurements and scanning protocols should be prioritized in order to acquire methodological sound data. Standardization of body composition measurements should consist of reaching consensus on which lumbar or thoracic level to assess (L3), which specific muscle groups to assess (total skeletal muscle area), and which contrast phase to use. Body composition assessment should be incorporated into the work-up of any patient undergoing $\mathrm{CT}$ or $\mathrm{MRI}$ in order to collect accurate data that might aid in the development of uniform thresholds for body composition parameters, shared decision making with regards to therapy, and develop more accurate treatment regimens for patients receiving systemic cytotoxic therapy.

The major drawback in defining cachexia or sarcopenia based on changes in body composition over time is that it cannot be used as a predictive factor. In order to intervene, we need to be able to predict which patients are at risk of losing skeletal muscle mass over time. The socalled radiomics approach might be able to assist in this prediction. CT-scans harbor a vast quantity of information which is invisible to the naked eye and which is therefore not used in regular assessments of body composition. These so-called radiomics features can be categorized into semantic and agnostic features. Semantic features such as shape, size, and location can be qualitatively defined by a radiologist. Agnostic features can be further divided 
into first, second, and higher order features. First order features examine gray level intensity of a region of interest independent of the spatial relationship with adjacent voxels. A voxel is a combination of volume and pixel, representing a value on a regular grid in a threedimensional space. Second order features examine the spatial relationship between gray level intensities. Higher order features aim to identify patterns within the image that are initially not perceivable or hard to interpret.[59,60] Radiomics features can be extracted using data processing algorithms and they can be used to predict treatment response or prognosis. [61] It is hypothesized that quantitative analysis of radiomics features derived from medical imaging data through (semi) automated software can provide better and more information than a physician.[61] They could possibly also be used to predict changes in body composition in the future.[62] If baseline radiomics features are able to predict changes in body composition, this might aid in early detection of deterioration of skeletal muscle and also be of use in shared decision making.

\section{Interventions}

Interventions for cancer cachexia are aimed at nutritional, exercise, or combined therapies. A recent systematic review on nutritional support in cancer-associated cachexia revealed inconclusive and conflicting results.[63] Agents such as eicosapentaenoic acid (EPA) have gained attention in the past years. EPA, an omega-3-fatty acid, favors production of prostaglandins and leukotrienes which are associated with a decreased inflammatory response and improved immunocompetence. Patients receiving oncological therapy and EPA showed preservation of lean body mass and weight when compared to patients receiving no EPA.[64-66] EPA has also been linked to suppression of the ubiquitin proteasome pathway, one of the principal mechanisms of protein catabolism in cachectic cancer patients. $[67,68]$ The effect of EPA suppletion on survival is unknown. Pre-clinical results show that amino acid enriched diets can promote protein synthesis[69, 70] and reduce protein breakdown in cachectic cancer patients.[71] Physical exercise is only effective when combined with protein supplementation in the post-exercise phase.[72] A recent Cochrane review classified the effectiveness of physical exercise therapy in cancer cachexia as uncertain. [73] However, it is promising that four randomized controlled trials on exercise interventions in cancer cachexia were identified whereas the previous Cochrane review identified none.[74] Although beneficial effects of exercise therapy on skeletal muscle mass in oncologic patients have been described [75], this did not necessarily reflect beneficial effects on skeletal muscle function.[76] A recent retrospective review of a multi-disciplinary clinical service for cancer cachexia with a multi modal approach consisting of medication, education, nutritional supplements, and exercise, revealed significant improvements in quality of life.[77] However,

clinical evidence in oncologic patients is very scarce. A randomized phase-II feasibility trial evaluating a multimodal approach (celecoxib, nutritional support, exercise) for cachexia in lung- and pancreatic cancer revealed very low compliance. Oral nutritional supplementation was only taken by $48 \%$ of patients, since it was not found palatable. There was no difference in skeletal muscle mass, physical function, or adverse events.[78] A phase III trial is now underway.[79] Although interventions for cachexia might be of clinical relevance, evidence is too scarce to come to definitive conclusions. 


\section{The gut microbiota}

Another interesting avenue to explore in the context of cancer cachexia is the gut microbiota. It is known that gut microbiota have a significant impact on host metabolism.[80] It is thought that gut barrier function, microbial defense, and Paneth cell function are disrupted because of cancer related inflammation, leading to dysbiosis. [81] Detrimental bacteria arising from this shift are thought to produce additional pro-inflammatory compounds which can be translocated along the disrupted gut barrier thereby contributing to systemic inflammation and cancer cachexia, leading to a vicious circle.[81] Recent pre-clinical data suggest that gut bacteria can contribute to the development of cancer cachexia. Reduction of systemic inflammation and expression of muscle atrophy markers was caused by oral supplementation of Lactobaccillus spp. in a mouse model of leukemia.[82] Evidence suggests direct implications of gut microbiota dysbiosis on skeletal muscle homeostasis. Colonization of the gut by an Escherichia coli strain prevented skeletal muscle atrophy in mice through sustained signaling of the insulin-like growth factor 1/phosphatidylinositol 3-kinase/AKT pathway.[83] Recently, it was shown that gut microbiota depletion in mice led to decreased running endurance and decreased muscle fatigue indices in ex vivo contractile testing. These effects were reversed by natural reseeding. It was hypothesized that these results were attributable to the impact of gut microbiota metabolites on glycogen availability and glucose metabolism in muscle.[84] Indeed, gut microbiota-derived phenolic compounds were found to promote glucose uptake in cultured differentiated human skeletal muscle myotubes in a dose dependent manner. [85] Gut microbiota composition is also known to influence the skeletal muscle fiber type. Oral supplementation of a Lactobacillus strain promoted the type I muscle fiber type in mice which is associated with higher muscle endurance and grip strength.[86] Additionally, microbial production of short chain fatty acids (SCFA) by gut bacteria can affect body weight gain through regulation of insulin sensitivity, energy harvesting, substrate metabolism, and energy intake.[87] There is mounting evidence that the gut microbiota affects anti-cancer treatment efficacy.[88] It was demonstrated that cyclophosphamide induced alterations of the gut microbiota and promoted selective translocation of Gram-positive species to secondary lymphoid organs. There they stimulated the generation of specific $T$ helper cells and immune responses. Germ-free mice and mice treated with antibiotics showed a reduction in the specific response and their tumors (sarcoma and lung adenocarcinoma) were resistant to chemotherapy.[89] Another study showed decreased response of subcutaneous tumors (lymphoma, colon carcinoma, melanoma) to immuno- and platinum based chemotherapy in mice with disrupted gut microbiota due to antibiotics.[90] Bacteria can directly influence drug metabolism through enzymatic degradation. Reduction, hydrolysis, alkylation, dihydroxylation, and many more metabolic processes are harbored by the gut microbiota which influence drug metabolism. Secondary metabolites produced by the gut microbiota can cause toxicity.[91] Since gut bacteria seem to be implicated in the development of cancer cachexia and reduced treatment efficacy, their role in ovarian cancer patients is in need of exploration. 


\section{Conclusion}

Body composition analyses can play an important role in the assessment of ovarian cancer patients at risk of adverse outcome. However, the heterogeneity in methods to assess body composition leads to conflicting outcomes. Additionally, conflicting results with regards to body composition and ovarian cancer survival are possibly due to shortcomings in the current definition of cachexia. The greatest challenges now lie in 1) identifying patients at risk; 2) establishing standardized methods for quantification of body composition; and 3) establishing new uniform criteria for cancer cachexia based on longitudinal data. Novel methods as proposed in PRIMs should aid in making an advanced prediction model for identifying patients at risk. Meanwhile, it is of pivotal importance to explore new possible therapeutic targets like the gut microbiota and to identify tumor mediated compounds that drive mechanisms behind cancer cachexia. For the latter, tumor-derived ascites provides for a new experimental approach. 


\section{References}

1. Mourtzakis, M., et al., A practical and precise approach to quantification of body composition in cancer patients using computed tomography images acquired during routine care. Appl Physiol Nutr Metab, 2008. 33(5): p. 997-1006.

2. Torres, M.L., et al., Nutritional status, CT body composition measures and survival in ovarian cancer. Gynecologic Oncology, 2013. 129(3): p. 548-553.

3. Aust, S., et al., Skeletal muscle depletion and markers for cancer cachexia are strong prognostic factors in epithelial ovarian cancer. PLoS ONE, 2015. 10 (10) (no pagination)(e0140403).

4. Rutten, I.J., et al., Loss of skeletal muscle during neoadjuvant chemotherapy is related to decreased survival in ovarian cancer patients. J Cachexia Sarcopenia Muscle, 2016. 7(4): p. 458-66.

5. Kumar, A., et al., Muscle composition measured by CT scan is a measurable predictor of overall survival in advanced ovarian cancer. Gynecologic Oncology, 2016. 142(2): p. 311-316.

6. Bronger, H., et al., Sarcopenia in Advanced Serous Ovarian Cancer. International Journal of Gynecological Cancer, 2017. 27(2): p. 223-232.

7. Rutten, I.J., et al., The influence of sarcopenia on survival and surgical complications in ovarian cancer patients undergoing primary debulking surgery. Eur J Surg Oncol, 2017. 43(4): p. 717-724.

8. Conrad, L.B., et al., Pre-operative core muscle index in combination with hypoalbuminemia is associated with poor prognosis in advanced ovarian cancer. Journal of Surgical Oncology, 2018. 117(5): p. 1020-1028.

9. Silva De Paula, N., et al., Sarcopenia and skeletal muscle quality as predictors of postoperative complication and early mortality in gynecologic cancer. International Journal of Gynecological Cancer, 2018. 28(2): p. 412-420.

10. Ataseven, B., et al., Skeletal Muscle Attenuation (Sarcopenia) Predicts Reduced Overall Survival in Patients with Advanced Epithelial Ovarian Cancer Undergoing Primary Debulking Surgery. Annals of Surgical Oncology, 2018. 25(11): p. 3372-3379.

11. Uccella, S., et al., Assessment of preoperative nutritional status using BIA-derived phase angle $(P h A)$ in patients with advanced ovarian cancer: Correlation with the extent of cytoreduction and complications. Gynecologic Oncology, 2018. 149(2): p. 263-269.

12. van Vugt, J.L.A., et al., Contrast-enhancement influences skeletal muscle density, but not skeletal muscle mass, measurements on computed tomography. Clin Nutr, 2018. 37(5): p. 1707-1714.

13. Rollins, K.E., et al., Body composition measurement using computed tomography: Does the phase of the scan matter? Nutrition, 2017. 41: p. 37-44.

14. Prado, C.M., et al., Sarcopenia as a determinant of chemotherapy toxicity and time to tumor progression in metastatic breast cancer patients receiving capecitabine treatment. Clin Cancer Res, 2009. 15(8): p. 2920-6.

15. Prado, C.M., et al., The association between body composition and toxicities from the combination of Doxil and trabectedin in patients with advanced relapsed ovarian cancer. Applied physiology, nutrition, and metabolism = Physiologie appliquee, nutrition et metabolisme, 2014. 39(6): p. 693-698.

16. Hirsch, L., et al., The impact of body composition parameters on severe toxicity of nivolumab. Eur J Cancer, 2020. 124: p. 170-177.

17. Ali, R., et al., Lean body mass as an independent determinant of dose-limiting toxicity and neuropathy in patients with colon cancer treated with FOLFOX regimens. Cancer Med, 2016. 5(4): p. 607-16. 
18. Karayiannakis, A.J., et al., Serum levels of tumor necrosis factor-alpha and nutritional status in pancreatic cancer patients. Anticancer Res, 2001. 21(2B): p. 1355-8.

19. Pfitzenmaier, J., et al., Elevation of cytokine levels in cachectic patients with prostate carcinoma. Cancer, 2003. 97(5): p. 1211-6.

20. Nakashima, J., et al., Association between tumor necrosis factor in serum and cachexia in patients with prostate cancer. Clin Cancer Res, 1998. 4(7): p. 1743-8.

21. Tisdale, M.J., Mechanisms of cancer cachexia. Physiol Rev, 2009. 89(2): p. 381-410.

22. Carson, J.A. and K.A. Baltgalvis, Interleukin 6 as a key regulator of muscle mass during cachexia. Exerc Sport Sci Rev, 2010. 38(4): p. 168-76.

23. Ramsey, M.L., et al., Circulating interleukin-6 is associated with disease progression, but not cachexia in pancreatic cancer. Pancreatology, 2019. 19(1): p. 80-87.

24. Baltgalvis, K.A., et al., Muscle wasting and interleukin-6-induced atrogin-l expression in the cachectic Apc ( Min/+ ) mouse. Pflugers Arch, 2009. 457(5): p. 989-1001.

25. Bonetto, A., et al., STAT3 activation in skeletal muscle links muscle wasting and the acute phase response in cancer cachexia. PLoS One, 2011. 6(7): p. e22538.

26. Bonetto, A., et al., JAK/STAT3 pathway inhibition blocks skeletal muscle wasting downstream of IL-6 and in experimental cancer cachexia. Am J Physiol Endocrinol Metab, 2012. 303(3): p. E410-21.

27. Prado, B.L. and Y. Qian, Anti-cytokines in the treatment of cancer cachexia. Ann Palliat Med, 2019. 8(1): p. 67-79.

28. Jatoi, A., et al., A placebo-controlled double blind trial of etanercept for the cancer anorexia/weight loss syndrome: results from NOOC1 from the North Central Cancer Treatment Group. Cancer, 2007. 110(6): p. 1396-403.

29. Jatoi, A., et al., A placebo-controlled, double-blind trial of infliximab for cancer-associated weight loss in elderly and/or poor performance non-small cell lung cancer patients (N01C9). Lung Cancer, 2010. 68(2): p. 234-9.

30. Wiedenmann, B., et al., A multicenter, phase II study of infliximab plus gemcitabine in pancreatic cancer cachexia. J Support Oncol, 2008. 6(1): p. 18-25.

31. Mankhong, S., et al., Experimental Models of Sarcopenia: Bridging Molecular Mechanism and Therapeutic Strategy. Cells, 2020. 9(6).

32. Onesti, J.K. and D.C. Guttridge, Inflammation based regulation of cancer cachexia. Biomed Res Int, 2014. 2014: p. 168407.

33. Yuan, L., et al., Muscle-specific E3 ubiquitin ligases are involved in muscle atrophy of cancer cachexia: an in vitro and in vivo study. Oncol Rep, 2015. 33(5): p. 2261-8.

34. Bodine, S.C. and L.M. Baehr, Skeletal muscle atrophy and the E3 ubiquitin ligases MuRF1 and MAFbx/atrogin-1. Am J Physiol Endocrinol Metab, 2014. 307(6): p. E469-84.

35. Ladner, K.J., M.A. Caligiuri, and D.C. Guttridge, Tumor necrosis factor-regulated biphasic activation of NF-kappa B is required for cytokine-induced loss of skeletal muscle gene products. J Biol Chem, 2003. 278(4): p. 2294-303.

36. Cella, P.S., et al., Creatine supplementation in Walker-256 tumor-bearing rats prevents skeletal muscle atrophy by attenuating systemic inflammation and protein degradation signaling. Eur J Nutr, 2020. 59(2): p. 661-669.

37. Talbert, E.E., et al., Modeling Human Cancer-induced Cachexia. Cell Rep, 2019. 28(6): p. 16121622 e4.

38. Op den Kamp, C.M., et al., Nuclear transcription factor kappa B activation and protein turnover adaptations in skeletal muscle of patients with progressive stages of lung cancer cachexia. Am J Clin Nutr, 2013. 98(3): p. 738-48.

39. Pettersen, K., et al., Cancer cachexia associates with a systemic autophagy-inducing activity mimicked by cancer cell-derived IL-6 trans-signaling. Sci Rep, 2017. 7(1): p. 2046.

40. Guigni, B.A., et al., Effects of conditioned media from murine lung cancer cells and human tumor cells on cultured myotubes. Am J Physiol Endocrinol Metab, 2020. 318(1): p. E22-E32. 
41. Jackman, R.W., et al., Continuous Release of Tumor-Derived Factors Improves the Modeling of Cachexia in Muscle Cell Culture. Front Physiol, 2017. 8: p. 738.

42. Zhang, G., B. Jin, and Y.P. Li, C/EBPbeta mediates tumour-induced ubiquitin ligase atrogin1/MAFbx upregulation and muscle wasting. EMBO J, 2011. 30(20): p. 4323-35.

43. Zhang, G., et al., Toll-like receptor 4 mediates Lewis lung carcinoma-induced muscle wasting via coordinate activation of protein degradation pathways. Sci Rep, 2017. 7(1): p. 2273.

44. Penson, R.T., et al., Cytokines IL-1beta, IL-2, IL-6, IL-8, MCP-1, GM-CSF and TNFalpha in patients with epithelial ovarian cancer and their relationship to treatment with paclitaxel. Int J Gynecol Cancer, 2000. 10(1): p. 33-41.

45. Cushen, S.J., et al., Body Composition by Computed Tomography as a Predictor of Toxicity in Patients With Renal Cell Carcinoma Treated With Sunitinib. Am J Clin Oncol, 2017. 40(1): p. 47-52.

46. Anandavadivelan, P., et al., Sarcopenic obesity: A probable risk factor for dose limiting toxicity during neo-adjuvant chemotherapy in oesophageal cancer patients. Clin Nutr, 2016. 35(3): p. 724-30.

47. Tan, B.H., et al., Sarcopenia is associated with toxicity in patients undergoing neo-adjuvant chemotherapy for oesophago-gastric cancer. Eur J Surg Oncol, 2015. 41(3): p. 333-8.

48. Du Bois D. Du Bois EF. Clinical calorimetry: tenth paper. A formula to estimate the approximate surface area if height and weight be known.Arch Intern Med (Chic). 1916; 17: 863-871

49. Gusella, M., et al., Relationships between body composition parameters and fluorouracil pharmacokinetics. Br J Clin Pharmacol, 2002. 54(2): p. 131-9.

50. Morgan, D.J. and K.M. Bray, Lean body mass as a predictor of drug dosage. Implications for drug therapy. Clin Pharmacokinet, 1994. 26(4): p. 292-307.

51. Sjoblom, B., et al., Drug Dose Per Kilogram Lean Body Mass Predicts Hematologic Toxicity From Carboplatin-Doublet Chemotherapy in Advanced Non-Small-Cell Lung Cancer. Clin Lung Cancer, 2017. 18(2): p. e129-e136.

52. Prado, C.M., et al., Prevalence and clinical implications of sarcopenic obesity in patients with solid tumours of the respiratory and gastrointestinal tracts: a population-based study. Lancet Oncol, 2008. 9(7): p. 629-35.

53. Crosby, V., et al., Can body composition be used to optimize the dose of platinum chemotherapy in lung cancer? A feasibility study. Support Care Cancer, 2017. 25(4): p. 12571261.

54. Fearon, K., et al., Definition and classification of cancer cachexia: an international consensus. Lancet Oncol, 2011. 12(5): p. 489-95.

55. van der Werf, A., et al., Percentiles for skeletal muscle index, area and radiation attenuation based on computed tomography imaging in a healthy Caucasian population. Eur J Clin Nutr, 2018. 72(2): p. 288-296.

56. van Vugt, J.L.A., et al., Estimated skeletal muscle mass and density values measured on computed tomography examinations in over 1000 living kidney donors. Eur J Clin Nutr, 2019. 73(6): p. 879-886.

57. Dodds RM, Syddall HE, Cooper R, Benzeval M, Deary IJ, Dennison EM, Der G, Gale CR, Inskip HM, Jagger C, Kirkwood TB, Lawlor DA, Robinson SM, Starr JM, Steptoe A, Tilling K, Kuh D, Cooper C, Sayer AA (2014) Grip strength across the life course: normative data from twelve British studies. PLoS One 9:e113637.

58. Eisenhauer, E.A., et al., New response evaluation criteria in solid tumours: revised RECIST guideline (version 1.1). Eur J Cancer, 2009. 45(2): p. 228-47.

59. Rizzo, S., et al., Radiomics: the facts and the challenges of image analysis. Eur Radiol Exp, 2018. 2(1): p. 36.

60. Gillies, R.J., P.E. Kinahan, and H. Hricak, Radiomics: Images Are More than Pictures, They Are Data. Radiology, 2016. 278(2): p. 563-77. 
61. Lambin, P., et al., Radiomics: extracting more information from medical images using advanced feature analysis. Eur J Cancer, 2012. 48(4): p. 441-6.

62. de Jong, E.E.C., et al., Can radiomics help to predict skeletal muscle response to chemotherapy in stage IV non-small cell lung cancer? Eur J Cancer, 2019. 120: p. 107-113.

63. Kim, A.J., D.S. Hong, and G.C. George, Diet-related interventions for cancer-associated cachexia. J Cancer Res Clin Oncol, 2021.

64. Ryan, A.M., et al., Enteral nutrition enriched with eicosapentaenoic acid (EPA) preserves lean body mass following esophageal cancer surgery: results of a double-blinded randomized controlled trial. Ann Surg, 2009. 249(3): p. 355-63.

65. van der Meij, B.S., et al., Oral nutritional supplements containing n-3 polyunsaturated fatty acids affect quality of life and functional status in lung cancer patients during multimodality treatment: an RCT. Eur J Clin Nutr, 2012. 66(3): p. 399-404.

66. Vasson, M.P., et al., Immunonutrition improves functional capacities in head and neck and esophageal cancer patients undergoing radiochemotherapy: a randomized clinical trial. Clin Nutr, 2014. 33(2): p. 204-10.

67. Fearon, K.C., et al., Effect of a protein and energy dense $\mathrm{N}-3$ fatty acid enriched oral supplement on loss of weight and lean tissue in cancer cachexia: a randomised double blind trial. Gut, 2003. 52(10): p. 1479-86.

68. Tisdale, M.J., The ubiquitin-proteasome pathway as a therapeutic target for muscle wasting. J Support Oncol, 2005. 3(3): p. 209-17.

69. Deutz, N.E., et al., Muscle protein synthesis in cancer patients can be stimulated with a specially formulated medical food. Clin Nutr, 2011. 30(6): p. 759-68.

70. Dillon, E.L., et al., Amino acid metabolism and inflammatory burden in ovarian cancer patients undergoing intense oncological therapy. Clin Nutr, 2007. 26(6): p. 736-43.

71. van Dijk, D.P., et al., Effects of oral meal feeding on whole body protein breakdown and protein synthesis in cachectic pancreatic cancer patients. J Cachexia Sarcopenia Muscle, 2015. 6(3): p. 212-21.

72. Atherton, P.J., et al., Muscle full effect after oral protein: time-dependent concordance and discordance between human muscle protein synthesis and mTORC1 signaling. Am J Clin Nutr, 2010. 92(5): p. 1080-8.

73. Grande, A.J., et al., Exercise for cancer cachexia in adults. Cochrane Database Syst Rev, 2021. 3: p. CD010804.

74. Grande, A.J., V. Silva, and M. Maddocks, Exercise for cancer cachexia in adults: Executive summary of a Cochrane Collaboration systematic review. J Cachexia Sarcopenia Muscle, 2015. 6(3): p. 208-11.

75. Focht, B.C., et al., Resistance exercise interventions during and following cancer treatment: $a$ systematic review. J Support Oncol, 2013. 11(2): p. 45-60.

76. Cermak, N.M., et al., Protein supplementation augments the adaptive response of skeletal muscle to resistance-type exercise training: a meta-analysis. Am J Clin Nutr, 2012. 96(6): p. 1454-64.

77. Kelcey A. Bland, et.al., Quality of life and symptom burden improve in patients attending a multidisciplinary clinical service for cancer cachexia: a retrospective observational review, Journal of Pain and Symptom Management, 2021, epub ahead of print.

78. Solheim, T.S., et al., A randomized phase II feasibility trial of a multimodal intervention for the management of cachexia in lung and pancreatic cancer. J Cachexia Sarcopenia Muscle, 2017. 8(5): p. 778-788.

79. Solheim, T.S., et al., Cancer cachexia: rationale for the MENAC (Multimodal-Exercise, Nutrition and Anti-inflammatory medication for Cachexia) trial. BMJ Support Palliat Care, 2018. 8(3): p. 258-265.

80. Sonnenburg, J.L. and F. Backhed, Diet-microbiota interactions as moderators of human metabolism. Nature, 2016. 535(7610): p. 56-64. 
81. Bindels, L.B. and N.M. Delzenne, Muscle wasting: the gut microbiota as a new therapeutic target? Int J Biochem Cell Biol, 2013. 45(10): p. 2186-90.

82. Bindels, L.B., et al., Synbiotic approach restores intestinal homeostasis and prolongs survival in leukaemic mice with cachexia. ISME J, 2016. 10(6): p. 1456-70.

83. Schieber, A.M., et al., Disease tolerance mediated by microbiome E. coli involves inflammasome and IGF-1 signaling. Science, 2015. 350(6260): p. 558-63.

84. Nay, K., et al., Gut bacteria are critical for optimal muscle function: a potential link with glucose homeostasis. Am J Physiol Endocrinol Metab, 2019. 317(1): p. E158-E171.

85. Houghton, M.J., et al., Gut microbiome catabolites as novel modulators of muscle cell glucose metabolism. FASEB J, 2019. 33(2): p. 1887-1898.

86. Chen, Y.M., et al., Lactobacillus plantarum TWK10 Supplementation Improves Exercise Performance and Increases Muscle Mass in Mice. Nutrients, 2016. 8(4): p. 205.

87. Canfora, E.E., et al., Colonic infusions of short-chain fatty acid mixtures promote energy metabolism in overweight/obese men: a randomized crossover trial. Sci Rep, 2017. 7(1): p. 2360.

88. Aarnoutse, R., et al., The Clinical Link between Human Intestinal Microbiota and Systemic Cancer Therapy. Int J Mol Sci, 2019. 20(17).

89. Viaud, S., et al., The intestinal microbiota modulates the anticancer immune effects of cyclophosphamide. Science, 2013. 342(6161): p. 971-6.

90. lida, N., et al., Commensal bacteria control cancer response to therapy by modulating the tumor microenvironment. Science, 2013. 342(6161): p. 967-70.

91. Alexander, J.L., et al., Gut microbiota modulation of chemotherapy efficacy and toxicity. Nat Rev Gastroenterol Hepatol, 2017. 14(6): p. 356-365. 


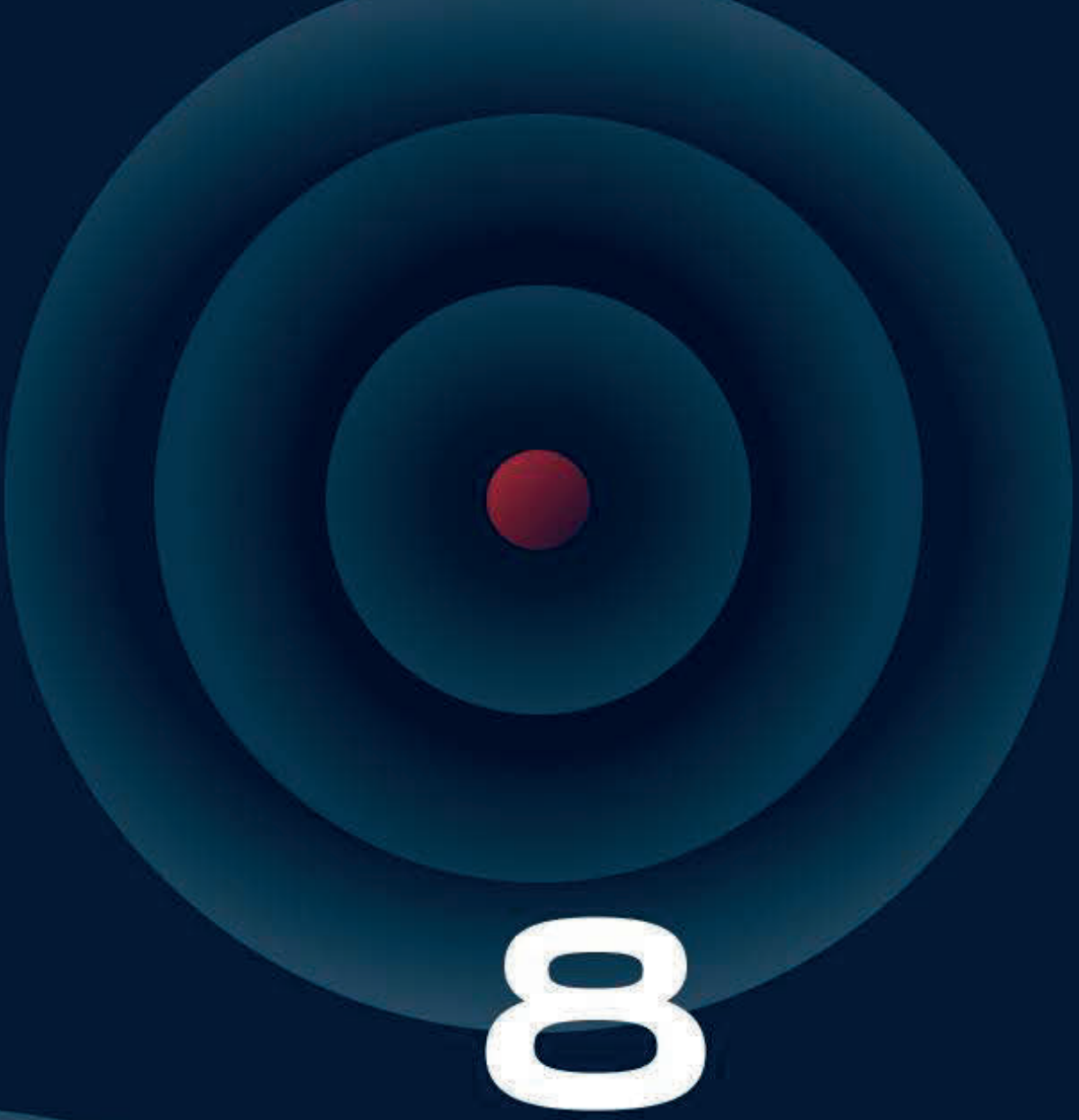


Summary 


\section{Summary}

Around three-quarters of all newly-diagnosed ovarian cancer patients present themselves with intraperitoneal or systemic metastasized disease. Since most patients have metastasized disease at first presentation overall five-year survival rates are poor. The main surgical and systemic treatments have not changed much over the past 25-years and therefore overall survival has neither. Recent developments have led to improvements in short term overall survival, but it is yet unclear what their effect on long term overall survival is. To explore new therapeutic targets the relationship between the tumor and the host (the patient) have been explored. Tumors can alter the metabolism of the host by influencing uptake of nutrients, demand for nutrients, and inflammation. This altered metabolism can lead to a syndrome called cancer cachexia. Cancer cachexia is highly prevalent in ovarian cancer patients and is associated with negative outcomes. This syndrome is characterized by weight loss, changes in body composition, functional decline and adverse psychological symptoms. Body composition analysis in cancer patients is most frequently performed with CT-scans. Out of all body composition parameters skeletal muscle is probably the most important one since it has been associated with functional decline and worse outcomes. A decline or low skeletal muscle mass is called sarcopenia and it is part of the cancer cachexia syndrome. Myosteatosis is frequently used for determining skeletal muscle quality and also associated with worse outcome in cancer patients. However, the drivers of cancer cachexia have not yet been fully elucidated and there is an ongoing debate on how to accurately perform body composition analysis with applicable cut-off values.

In chapter $\mathbf{2}$ we provide a systematic overview of all available literature on the subject of body composition and its association with outcome in ovarian cancer patients. Ten studies were identified in which skeletal muscle was related to outcome in ovarian cancer patients. Eight of these studies were included in the meta-analysis for outcome. Sarcopenia and myosteatosis were significantly associated with worse overall survival in ovarian cancer patients. Sarcopenia was associated with the development of surgical complications with borderline significance. Sarcopenia and myosteatosis are related to outcome in ovarian cancer patients. However, the high risk of bias and low quality of the source data makes it difficult to come to a definitive conclusion.

In chapter $\mathbf{3}$ we determined whether total skeletal muscle area measurements (CT-scan) can be substituted with psoas area measurements only. Patients $(n=150)$ with ovarian cancer who had undergone neo-adjuvant chemotherapy were included. Pre- and post-chemotherapy body composition analysis was performed on the abdominal CT-scan. Total skeletal muscle area was compared to psoas muscle area and psoas length and width. The correlation between total skeletal muscle area, psoas area and psoas length and width was poor. Patients were divided in muscle loss and gain groups. Kappa agreement between al methods was also 
poor. Psoas muscle measurements should not be used to substitute total skeletal muscle area measurements

In chapter 4 we assessed whether low baseline skeletal muscle was associated with worse outcome in ovarian cancer patients $(n=216)$ undergoing primary debulking surgery. Body composition analysis was performed on the pre-operative CT-scan. Instead of using a previously published cut-off for determining sarcopenia we performed optimum stratification analysis for determining a cut-off. Sarcopenia was significantly associated with worse overall survival in univariate analysis but the effect was lost in multivariate analysis. The completeness of surgery, treatment in a specialized centre, and occurrence of a major complication were stronger predictors for outcome. Myosteatosis was significantly associated with the development of surgical complications in univariate analysis, but the effect was lost again in multivariate analysis. Although a strong trend between sarcopenia, myosteatosis, and worse outcome was found in this cohort, there were other stronger predictors for outcome.

In chapter $\mathbf{5}$ we aimed to validate the previously published finding that a decrease in skeletal muscle index during neoadjuvant chemotherapy in ovarian cancer patients leads to worse overall survival. Body composition analysis was performed on abdominal CT-scans of patients $(n=212)$ who participated in the OVHIPEC randomized controlled trial. All patients had FIGO stage III disease and similar performance score and they were prospectively enrolled, making this the most homogeneous cohort to date. Patients underwent neoadjuvant chemotherapy and were eligible for interval debulking surgery. CT-scans were performed before and after two cycles of neoadjuvant chemotherapy. The difference in SMI between both scans was related to overall survival, recurrence free survival and number of adverse events. The cohort was divided into two groups based on a previously published cut-off of $2 \%$ decrease in SMI per 100 days. Median overall survival and median recurrence free survival was not different between both groups. Mean and total number of adverse events was higher in the groups of patients who lost skeletal muscle mass when compared to the patients who maintained or gained muscle mass.

In chapter 6 we aimed to establish a model for ovarian cancer cachexia to isolate factors responsible for skeletal muscle breakdown. Patients with suspected malignancy of the ovary $(n=15)$ who had not yet undergone treatment were prospectively enrolled. Extensive physical screening was performed before treatment and ascites and blood were collected. Body composition analysis was performed on abdominal CT-scans. Based on body composition analysis and histopathologic outcome patients were divided into three groups: sarcopenia, no sarcopenia and benign control. Ascites was collected and concentrations of cachexiaassociated factors were assessed by ELISA. Subsequently, ascites was used for in vitro exposure of $\mathrm{C} 2 \mathrm{C} 12$ myotubes followed by measurements of protein synthesis and breakdown by radioactive isotope tracing, qPCR-based analysis of atrophy-related gene expression, and NF-kB activity reporter assays. Ascites of sarcopenic ovarian cancer patients induces 
pronounced skeletal muscle protein metabolism changes in $\mathrm{C} 2 \mathrm{C} 12$ cells that correlate with clinical muscle measures of the patient and that are characteristic of cachexia. The use of ascites offers a new experimental tool to study the impact of both tumor-derived and systemic factors in various cachexia model systems, enabling identification of novel drivers of tissue wasting in ovarian cancer. 
Samenvatting 


\section{Samenvatting}

Ongeveer driekwart van de patienten met een ovariumcarcinoom presenteert zich met intraperitoneale of gemetastaseerde ziekte. Omdat het gros van de patienten zich presenteert met gemetastaseerde ziekte bij de eerste presentatie is de vijfjaarsoverleving slecht. De hoeksteen van de behandeling bestaat uit chirurgie en aanvullende chemotherapie. De behandelingopties zijn de afgelopen 25 jaar weinig veranderd en daarmee ook de overleving. Recente ontwikkelingen hebben geleid tot een verbeterde overleving op korte termijn, maar hun effect op de lange termijn is nog onzeker. Om nieuwe behandelingsopties te verkennen is de relatie tussen de tumor en de patient onderwerp van onderzoek geworden. Tumoren kunnen het metabolisme van een patient beinvloeden en spelen een rol in opname van voedingsstoffen, vraag naar voedingsstoffen en inflammatie. Deze beinvloeding van het metabolisme door de tumor kan leiden tot een syndroom dat kankercachexie heet. Cachexie is veelvoorkomend bij patienten met een ovariumcarcinoom en geassocieerd met slechte uitkomsten. Het syndroom kenmerkt zich door gewichtverlies, veranderingen in lichaamssamenstelling, afname van fysieke functie en psychologische symptomen. Veranderingen in lichaamssamenstelling worden bij patienten met kanker waargenomen door middel van CT scans. Van alle waarneembare compartimenten is skeletspier de meest belangrijke en geassocieerd met slechtere uitkomstmaten en afname van fysieke functie. Een afname van skeletspiermassa of een lage skeletspiermassa wordt sarcopenie genoemd, en sarcopenie is onderdeel van het syndroom cachexie. Myosteatose is een term die wordt gebruikt om vervetting van skeletspieren aan te duiden. Vervetting van de spieren is ook geassocieerd met slechtere uitkomstmaten bij patiente met kanker. Echter, welke processen er precies een rol spelen bij het ontstaan van cachexie en sarcopenie is nog niet helemaal duidelijk. Voorts is het een onderwerp van discussie hoe metingen van lichaamssamenstelling met behulp van CT scans het best verricht kunnen worden en welke afkapwaarden er gebruikt moeten worden.

In hoofdstuk 2 wordt er een systematisch overzicht van alle beschikbare wetenschappelijke literatuur over de relatie tussen skeletspiermassa en uitkomstmaten bij patienten met een ovariumcarcinoom weergegeven. Tien studies welke de relatie tussen skeletspiermassa en uitkomstmaten zoals overleving en complicaties van de behandeling hadden onderzocht bij patienten met een ovariumcarcinoom werden beschreven. Sarcopenie en myosteatose waren significant geassocieerd met slechtere overleving in patienten met een ovariumcarcinoom. Sarcopenie was ook gerelateerd aan het ontwikkelen van chirurgische complicaties. Echter, de lage kwaliteit van de brondata en hoge risico op bias maakt het moeilijk om tot een definitieve conclusie te komen.

In hoofdstuk 3 wordt beschreven of metingen van het totale skeletspieroppervlak middels een CT scan vervangen kunnen worden door metingen van alleen de psoas spier. Patienten $(n=150)$ met een ovariumcarcinoom welke neo-adjuvante chemotherapie ondergingen 
werden meegenomen in dit onderzoek. Voorafgaand aan de behandeling en nadien werd er een CT scan verricht waarmee de lichaamssamenstelling van de patienten werd berekend. Het totale oppervlak van de skeletspieren werd vergeleken met het oppervlak de psoas spier en de lengte $x$ breedte van de psoas spier. De correlatie tussen de verschillende metingen was slecht. Patienten werden verdeeld in twee groepen. Een groep liet een afname van de spiermassa zien, de andere groep bleef stabiel of liet een toename van de spiermassa zien. De kappa statistiek tussen de vergeleken methoden liet blijken dat de metingen slecht overeenkwamen. Een afname van de totale skeletspiermassa was geassocieerd met een slechtere overleving. Metingen van de psoas spier moeten niet gebruikt worden voor metingen van de skeletspiermassa.

In hoofdstuk 4 wordt onderzocht of sarcopenie vastgesteld voorafgaand aan primaire debulking chirurgie gerelateerd is aan slechtere uitkomstmaten bij patienten $(n=216)$ met een ovariumcarcinoom. Middels CT scan werd de lichaamssamenstelling geanalyseerd voorafgaand aan de operatie. Optimum stratificatie analyse werd toegepast om een afkapwaarde voor een lage spiermassa te bepalen. Sarcopenie was geassocieerd met overleving in univariate analyse, maar het effect ging verloren in multivariate analyse. Resterend tumor residue na de operatie, behandeling in een oncologisch centrum en het optreden van een grote complicatie waren betere voorspellers voor overleving. Myosteatose was geassocieerd met het ontstaan van chirurgische complicaties in univariate analyse, maar ook dit effect ging verloren in multivariate analyse. Concluderend was er een trend zichtbaar voor de relatie van sarcopenie en myosteatose met overleving en complicaties. Echter, er waren krachtigere voorspellers voor overleving.

In hoofdstuk 5 werd getracht de eerder geobserveerde associatie tussen een afname van spiermassa en slechtere overleving bij patienten met een ovariumcarcinoom die neoadjuvante chemotherapie ondergingen te bevestigen. Analyse van lichaamssamenstelling werd verricht op abdominale CT scans van patienten $(n=212)$ die hadden deelgenomen aan het OVHIPEC onderzoek. Omdat alle patienten prospectief werden geincludeerd en daarom allemaal een FIGO stadium 3 ovariumcarcinoom hadden evenals een vergelijkbare performance score was dit het meest homogene cohort tot nu toe. Patienten ondergingen neo-adjuvante chemotherapie en kwamen nadien in aanmerking voor interval debulking chirurgie. CT scans werden voorafgaand en na twee cycli kuren chemoterapie verricht. Het verschil in berekende spiermassa tussen beide scans werd gerelateerd aan overleving, ziektevrije overleving en het aantal geobserveerde adverse events. In het eerder gepubliceerde cohort werden patienten verdeeld in twee groepen gebaseerd op een stabiele of toenemende spiermassa vs. afname met een foutmarge van $2 \%$. De afkapwaarde was daarom in dit cohort $2 \%$ of meer afname van skeletspiermassa per 100 dagen. Mediane overleving en mediane ziektevrije overleving was niet verschillend tussen beide groepen. 
Echter, er was een sterke associatie tussen het gemiddelde en totale aantal adverse events en een afname van de spiermassa.

In hoofdstuk 6 wordt een model beschreven om factoren die een rol spelen in de ontwikkeling van cachexie bij het ovariumcarcinoom te kunnen isoleren. Patienten $(n=15)$ die verdacht werden van een maligniteit van het ovarium en nog geen behandeling hadden ondergaan werden prospectief geincludeerd. Voorafgaand aan hun behandeling werden patienten uitgebreid fysiek gescreend en werd er bloed en ascites afgenomen. Analyse van lichaamssamenstelling werd verricht op abdominale CT scans. Gebaseerd op analyse van lichaamssamenstelling en histopathologie werden patienten verdeeld in de drie groepen; sarcopeen, niet-sarcopeen en benigne controle. Concentraties van cachexie geassocieerde factoren in ascites werden bepaald middels ELISA. Ascites werd gebruikt voor in vitro blootstelling van skeletspiercellen om eiwitsynthese en afbraak te meten door middel van tracing met radioactieve istopen, expressie van atrofie gerelateerde genen door middel van qPCR en NF-kB activiteit middels reporter assays. Ascites afkomstig van sarcopene patienten induceerde uitgesproken veranderingen in eiwitmetabolisme welke correleren met de klinische uitkomstmaten van de patienten en die karakteristiek zijn voor cachexie. Het gebruik van ascites biedt een nieuwe experimentele opzet voor het bestuderen van cachexie en kan helpen met het identificeren van factoren verantwoordelijk voor het ontstaan van cachexie bij het ovariumcarcinoom. 

Impact paragraph 


\section{Introduction and results}

Ovarian cancer is a highly lethal malignancy. Around 1300 women are diagnosed with ovarian cancer on a yearly basis in the Netherlands, with an approximate mortality of around 1000 . Treatment consists of surgery supplemented with chemotherapy. Although there have been some improvements in survival rates, chances for cure remain slim. Five-year survival is estimated at $20-60 \%$ for advanced stage ovarian cancer. Therefore, the search for therapeutic targets remains ongoing. The patients' metabolic phenotype has recently gained considerable attention in this context. It was recently seen that ovarian cancer patients who lost skeletal muscle during chemotherapy experience shorter overall survival. Although these are intriguing results, the potential causality between diminishing skeletal muscle tissue and worse overall survival has yet to be determined. Additionally, guidelines or protocols for determining skeletal muscle mass and quality based on radiological imaging were lacking.

This dissertation focuses on body composition measurements and on unravelling skeletal muscle pathophysiology in ovarian cancer patients. The aim of this dissertation was to gain more insight into the association between skeletal muscle measurements and outcome in ovarian cancer patients undergoing surgery and/or chemotherapy. Patients undergoing primary debulking surgery with low baseline skeletal muscle mass as seen on CT-scan experienced worse overall survival when compared to patients with normal skeletal muscle mass. Although previous results showed a clear survival disadvantage for ovarian cancer patients losing skeletal muscle during neoadjuvant chemotherapy, these results were not reproducible in the largest validation cohort to date. However, an interesting association between diminishing skeletal muscle mass and adverse events was detected. A review and meta-analysis confirmed the association between low skeletal muscle index and ovarian cancer survival. However, the validity of this conclusion was hampered by the heterogeneity in used measurement protocols and cut-offs for body composition precluded drawing definitive conclusions. Evidence produced in this thesis provided a sound method for calculating skeletal muscle using CT-scanning. Efforts were made to elucidate mechanisms underlying skeletal muscle tissue loss and ovarian cancer outcome. Cultured skeletal muscle cells were exposed to ascites derived from ovarian cancer patients. Cells exposed to ascites derived from cachectic patients had a lower capacity for protein synthesis. CT-scans were supplemented with extensive physical screening to distinguish cachectic from non-cachectic patients. Data extracted from the physical fitness screening revealed several intriguing correlations between radiological imaging and experimental outcomes. These results underline the importance of complementing radiological imaging with screening for physical fitness. 


\section{Scientific impact}

Based on data provided in this thesis, it was proposed to use total skeletal muscle area at the third lumbar vertebra on CT-scans to assess skeletal muscle in ovarian cancer patients. These results were highly cited (73 times) since their publication. This method of assessment has since been widely accepted as a valid way of estimating skeletal muscle mass in (ovarian) cancer patients. Other results of this thesis contributed to determining the relationships between skeletal muscle mass and ovarian cancer survival. It was proposed to use changes in skeletal muscle mass during neo-adjuvant chemotherapy to distinguish sarcopenic from nonsarcopenic patients instead of single measurements in time with a clearly defined cut-off. Although a prospective validation study failed to reproduce a relationship between overall survival and skeletal muscle, a strong correlation between skeletal muscle mass and chemotherapy related toxicity was observed. Based on the observed correlation between skeletal muscle mass and toxicity a future trial should investigate the dosing of chemotherapy based on body composition and its potential impact on the prevention of dose limiting toxicities, thereby translating scientific results to clinical practice. Additionally, skeletal muscle protein synthesis in cultured skeletal muscle cells was negatively affected by exposure to ascites from ovarian cancer patients, introducing a new and accessible tool in translational cachexia research. This approach will shed new light on how skeletal muscle aberrations may originate in cancer patients, since the known genes in skeletal muscle catabolism were not affected by factors in ascites. All the results of this thesis were presented at international conferences and were published in internationally peer reviewed journals.

\section{Societal impact}

Cancer cachexia is a severe and debilitating condition which severely impacts quality of life, survival, treatment efficacy, and psychosocial symptoms and distress. This thesis has revealed relationships between low skeletal muscle mass and impaired outcome in ovarian cancer patients. These outcomes are highly relevant to ovarian cancer patients and their physicians. Body composition analyses could serve as a tool for identifying patients at risk of adverse outcomes in the future work-up of ovarian cancer patients. Body composition measurements could be used for calculating dose administration for systemic cytotoxic therapy and for better shared decision-making for therapy. They could additionally serve as a gateway to improve peri-operative intervention programs, the likes of which have already been implemented in several healthcare institutions. Special attention should be directed at the psychosocial impact of cachexia in ovarian cancer patients. Although very prevalent in cancer patients in general, cachectic patients are even more affected by the psychological and physical aspects of their disease. In conclusion, body composition measurements are not only useful in the context of developing anti-tumor treatment strategies but also have an important role in defining patients at risk of developing adverse psychosocial symptoms and low quality of life related 
to their disease. Hence, this thesis also serves to raise awareness about cancer cachexia and its debilitating effects on ovarian cancer patients.

Cancer cachexia also has a profound societal impact. Recent analyses have shown that (cancer) cachexia carries a heavy burden on healthcare costs and hospitalization. Length of stay for cachectic patients was twice as high in comparison to non-cachectic patients, and costs of hospitalization were increased with 4600 euros per hospitalization. In this age of everincreasing healthcare expenditure, reduction of costs requires special attention. Intervention programs might aid in reducing length of stay and decrease healthcare expenditure for cachectic patients. The methods described in this thesis will aid in distinguishing patients eligible for intervention programs.

\section{Activity}

The results of this thesis provided a blueprint for a prospective study evaluating skeletal muscle fluctuations and adverse events in ovarian cancer patients receiving chemotherapy, thereby reaching out to healthcare professionals and patients alike. Efforts have been put into raising awareness and attention to the issue of cancer cachexia. The need for awareness and the effect of intervention studies was addressed in the recent 'kankerkachexie, het belang van het behoud van functie' symposium, where results of this thesis were also presented. Physicians, physician assistants, movement scientists, physiotherapists, and other paramedical staff participated in the symposium. A multidisciplinary approach of addressing cancer cachexia seems to be the way forward. Preservation and maintaining physical functioning, sensory functioning, and personalized medicine in oncologic patients were also embraced by the MUMC+ future perspectives which were addressed in the 'Gezond leven 2025 ' article. Since the inception of the "Journal of Cachexia, Sarcopenia, and Muscle" in 2010, which focuses on wasting diseases, the number of publications on (cancer) cachexia rose significantly. Its annual conference gained the topic of wasting diseases in oncology significant attention. Results from this thesis were presented on a yearly basis at this conference. However, translation of pre-clinical results to clinical intervention trials is still lacking, a statement which was also expressed by the society on "Cachexia, Sarcopenia, and Wasting Disorders". The PRIMs protocol presented in this thesis is a step in the right direction. However, more efforts could be made towards the inception of new trials and communication towards patients and patient organizations. Much is potentially to be gained in terms of healthcare expenditure, quality of life, psychosocial functioning, and survival of cancer patients. Therefore, the issue of wasting disease in oncology will hopefully gain more attention from patient organizations and healthcare providers as well as insurance companies. Researchers should meanwhile continue addressing the relevance of cancer cachexia. 
Chapter 9 | 155 


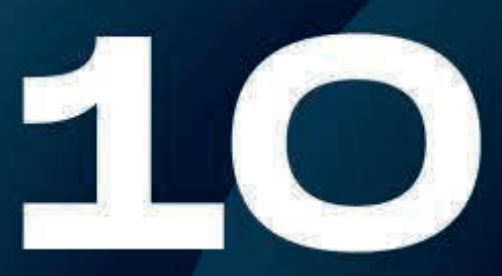


Epilogue 


\section{Dankwoord}

Met dit dankwoord komt er een einde aan mijn promotietraject. Met recht het einde, omdat ik het dankwoord als laatste heb bewaard. Het dankwoord is volgens sommige van mijn collega's het belangrijkste hoofdstuk uit het hele 'boekje', aangezien dit het enige hoofdstuk is dat door iedereen gelezen wordt. Ik weet niet of dat helemaal waar is. Ik denk wel dat het dankwoord het leukste hoofdstuk is om te schrijven. Het is een mooie terugblik op alle prachtige momenten en alle fantastische collega's die ik heb leren kennen tijdens de afgelopen jaren. Ik heb ontzettend veel geleerd en wil iedereen die de totstandkoming van dit proefschrift mede heeft gerealiseerd heel erg bedanken.

Professor Kruitwagen, beste Roy, de eerste promotor. Altijd stond je deur open. De afgelopen jaren ging je naast klinische taken ook managementtaken op je nemen. Dat moet onvoorstelbaar druk zijn. En toch, als ik je nodig had, dan had je altijd tijd voor me. Ik kon op elk willekeurig moment bij je binnenlopen met de meest triviale vragen en jij zorgde dan dat het direct geregeld werd. Je was ook heel erg praktisch ingesteld, ongetwijfeld door je jarenlange wetenschappelijke ervaring. Deadlines werden goed bewakkt (gelukkig maar!) en je zorgde ervoor dat ik het hele project gefocust bleef. Daarnaast heb je altijd in mij geloofd en ben je mij blijven steunen, ook toen ik het MUMC reeds verlaten had, bedankt daarvoor!

Professor Olde Damink, beste Steven, ik zal de eerste keer dat ik je kantoor verliet nooit vergeten. Het duizelde mij van alle plannen. Geen idee was te wild. Waar anderen moeilijkheden verwachtten, zag jij kansen. Ondanks je drukke bestaan maakte je altijd tijd vrij voor mij en toonde je steevast oprechte interesse in mij. Tijdens een voortgangsgesprek vroeg je aan mij hoe het met mij ging, waarop ik de voortgang van het project begon op te dreunen. Daarop zei je, "ik bedoel hoe het met jou gaat". En dat is natuurlijk ook veel belangrijker. Naast je drukke bezigheden was er ook genoeg tijd voor ontspanning. Zo was er de jaarlijkse PhD barbecue op je landgoed en heb ik met Lindsay mogen genieten van een weekend op je Alpaca's passen. Je bent een onuitputtelijke bron van energie en ik hoop dat je je oprechte interesse in de mensen om je heen nooit verliest. Bedankt voor je steun!

Professor Van Gorp, beste Toon, ik leerde je kennen in het derde jaar van de geneeskundeopleiding. Je was mijn mentor tijdens het blok 'abdomen'. Ik heb ontzettend veel van je geleerd. Wat was ik onder de indruk toen ik een keer mocht meekijken tijdens een robot Wertheim. Daarnaast zal ik me altijd blijven herinneren hoe je voortdurend het belang van de patiënt vooropstelde. Nadien zouden onze wegen zich nog enkele keren kruisen. Jij was degene die voorstelde om mijn geneeskundeopleiding vervolg te gaan geven middels een promotietraject. Wat vond ik het jammer dat je naar Leuven vertrok! Jouw kritische blik was van onschatbare waarde. Toon, bedankt voor alles.

Doctor Rensen, beste Sander, last but not least. Er wordt in dit dankwoord over veel mensen geschreven hoe belangrijk hun bijdrage was, maar zonder jouw hulp zou het echt een lastig 
verhaal zijn geworden! Je onuitputtelijke kennis was ontzettend waardevol voor de voltooiing van dit proefschrift. Toen ik in het laboratorium begon, wist ik net wat het 'business-end' van een pipet was. Als ik een manuscript terugkreeg van jou, was er vaak weinig van de originele tekst over. Dit leidde er echter wel toe dat het verrichte werk onder jouw supervisie uiteindelijk kwalitatief beter werd. Toen ik twijfelde of ik een huis moest kopen, zei je dat ik ervoor moest gaan en dat ik van zo'n belangrijke stap in mijn leven moest genieten. Je verloor nooit uit het oog wat er echt belangrijk is in het leven. Dankjewel voor alles!

Ik wil ook graag de leden van de leescommissie hartelijk bedanken voor de tijd en de energie die zij hebben gestoken in het kritisch beoordelen van mijn proefschrift. Prof. dr. Wildberger, prof. dr. Nijman, prof. dr. De Jong, dr. Lalisang en prof. dr. Van de Vijver.

Sjoerd en Denise, bedankt dat jullie mijn paranifmen willen zijn. Sjoerd, ik kwam je de eerste keer tegen in India en je dacht dat ik een Indiër was. Ik was nogal gebruind en had me een tijd niet geschoren. Dat was het begin van een mooie vriendschap. Latere reizen zouden ons nog langs Bolivia, Chili en Peru brengen, en minder ver maar niet minder mooi, het Oktoberfest! Altijd was er tijd voor een biertje om even stoom af te blazen. Bedankt maat voor de mooie avonturen! Denise, ik herinner me onze eerste ontmoeting nog goed. Plotseling een nieuw gezicht in het 'hok' van onderzoekers. We zouden uiteindelijk ruim drie jaar samen doorbengen. Drie jaren waarin ik heb gezien dat jij altijd voor iedereen klaarstond. In het begin was je bang dat je de kamer moest delen 'met een over het paard getild figuur' (jouw eigen woorden). Gelukkig bleek niks minder waar (ook jouw eigen woorden). Wat hebben we een lol gehad. Lief en leed werd er gedeeld en ik had met niemand liever de kamer willen delen. Lieve Denise, dankjewel!

Iris en Evelyne, wat keek ik tegen jullie op! Iris, jij was mijn dagelijkse begeleider tijdens mijn wetenschappelijke afstudeerstage. Bedankt voor de fijne begeleiding en de kansen die je mij hebt gegeven. Evelyne, jij was al een flink eind op weg met je promotie toen ik begon. Dank voor je hulp en gezelligheid! Wat hebben jullie de lat ontzettend hoog gelegd voor jullie opvolgers! Dankzij jullie beider begeleiding heb ik een vliegende start met mijn promotie kunnen maken. Veronique, Esther, Laura en Emma, soms lunchten we samen, soms deelden we een kamer, helaas te weinig omdat we zo verspreid door heel het gebouw zaten. Natuurlijk lieten we ons daardoor niet uit het veld slaan en volgden er regelmatig borrels, barbecues en etentjes. Mooi was ook de sushi avond waarop Robert-Jan sushi rollen maakte die zo groot waren als een rol keukenpapier. Pim en Robert-Jan, de mannelijke sterkhouders in het vrouwenbastion. Hopelijk volgen er nog veel mooi speciaalbieravonden.

Beste Gaby, altijd staat je deur open voor iedereen. De belangrijkste gesprekken werden bij jou aan je bureau gevoerd. De liefde, het kopen van een huis, alles kon er besproken worden. Altijd heb je oog voor je medewerkers en vind je het belangrijk dat zij goed in hun vel zitten. Gaby, bedankt voor je hulp en de fijne gesprekken. 
Monique, Petra, Trudy en Manon. De stille drijvende krachten achter het Vrouw, Moeder, Kind centrum. Hoe vaak heb ik jullie hulp nodig gehad. Jullie zijn onmisbaar! Geen vraag te groot of te klein. Soms was het om de professoren op hetzelfde tijdstip in dezelfde kamer te krijgen voor overleg (geen gemakkelijk klus!), soms waren de nietjes op, soms kwam ik drop stelen uit Trudy's pot en soms kwam ik gewoon even binnenwandelen om een praatje te maken. Bedankt voor de fijne tijd en jullie hulp!

Collega's van het cachexiegroepje. Rianne, dankjewel dat je mij op sleeptouw hebt genomen in het lab en alle ins en outs hebt geleerd van de celkweken. Heel veel succes met je post-doc! Merel, ik ken weinig mensen met zo'n uitgesproken mening als jij, dat wordt erg gewaardeerd! Succes met de voltooiing van je proefschrift. Gregory, excuses dat ik je tijdens een congres in Praag slapeloze nachten heb bezorgd met mijn gesnurk (en een scheet als ik je moet geloven). Het was een mooie tijd! Marjolein, bedankt voor de fijne samenwerking met het PRIMs project, succes met de voltooiing. David, de godfather van de body composition analyses. Bedankt voor alles wat je me hebt geleerd.

Collega's van de chirurgie: Annet, bedankt voor de gezelligheid, we hebben elkaar gevonden in onze voorliefde voor speciaalbier en gamen. Inmiddels heb je een PS5, daar ben ik best een beetje jaloers op en ik kom hem graag een keer uitproberen $-\mathcal{-}$. Mirjam, kamergenoot van Rianne en Annet, het was gezellig, met name altijd stiekem even roddelen op jullie kamer. Succes met de voltooiing van je proefschrift. Rob, een van de weinige mannen op het lab. Ik kon heel erg genieten van je humor. Zita, bedankt voor je hulp bij de analyses van de microbioom studie! Ralph, bedankt voor de gezelligheid en bijdrage aan de radiomics. Ik kom graag nog een keer een biertje drinken in je nieuwe huis als je kids me niet helemaal natspuiten met hun waterpistolen $(-)$. Romy, heel erg bedankt voor de fijne samenwerking en het vele werk dat je hebt verricht voor de microbioomstudie! Alle andere collega's van de chirurgie: Sara, Loes, Cathelijne, Evie, Caitlin, Kim, Renée, Aurelia, Anne, Kees, Jos, Patrick, Jacqueline, Remon, Tom, Ilse, Kiran, Claire, Dennis, Junfang, Lin, Xinwei, Hong, Gloria, Lara, Anjali, Chantal, Cathy, Tessa en Min heel erg bedankt voor de mooie tijd!

Lieve collega's uit Venlo, de plek waar ik mijn eerste klinische functie ging beoefenen, het was een bewogen periode. Ik wil jullie bedanken voor de steun en de fijne en gezellige werksfeer.

Bas, Mo, Hans en Annemarie, de drijvende krachten achter het laboratorium. Vanuit jullie kantoor waken jullie over het laboratorium zodat alle onervaren studenten en PhD kandidaten er geen puinhoop van maken. Ook jullie deur stond altijd open, en dat was ook nodig. Jullie expertise was onmisbaar bij de voltooiing van dit proefschrift. De koffieautomaat net voor jullie kantoor was regelmatig de ontmoetingsplek voor een vlotte babbel en om het weekend even door te nemen, te sparren over resultaten of flink te ventileren. Wanneer ik te laat aansloot bij de koffie was de bulderende lach van Mo al aan het begin van de gang te horen. Bedankt voor alles! 
Ramon, Wouter, Kenneth, Ruth, Sandrijne, Simone, Willemien, Gabe, Ardy, Brigitte en Jos, jullie wil ik bedanken voor het delen van jullie expertise, mentoring, en alle praktische hulp die jullie hebben geboden bij de voltooiing van dit proefschrift.

Janine, wat was jij een ontzettend goede student. Alles liep bij jou van een leien dakje. Altijd perfect voorbereid, goede kennis, secuur en vriendelijk. Daarnaast was je ook altijd wel te porren voor een lekker kopje koffie. Al je harde werk resulteerde niet alleen in enkele publicaties, maar ook in je eigen promotietraject. Dat had je ook verdiend! Heel erg bedankt voor je harde werk en hulp. Jacco, je komt soms mensen tegen in het leven waarvan je in een oogopslag weet dat je wel samen door een deur kunt. Jij was zo iemand. Eigenlijk was ik niet je begeleider, maar we hebben toch een mooie tijd gehad! We deelden een voorliefde voor speciaalbier en Roda JC en dat resulteerde in menig brakke ochtend. Het was niet alleen maar lol, want ook jij hebt laten zien dat je een ontzettend goede student was met inmiddels je eigen promotietraject!

Sander, Jim, Marijn, Marnik, Raymond, Rick, Thomas, Tobias, Tyas, de mannen van het 'weekendje'. We hebben elkaar leren kennen in het eerste jaar van de geneeskundeopleiding. Inmiddels is iedereen in opleiding, en sommigen zelfs al klaar met hun opleiding. Wat hebben we een mooie groep. In de loop der jaren sloten er regelmatige nieuwe mensen aan, die gemakkelijk integreerden in onze groep. Ondanks het drukke bestaan vinden we nog regelmatig de tijd voor een borreltje in, wat inmiddels misschien wel ons stamcafé genoemd mag worden, de Poshoorn. Onze jaarlijkse traditie van een of meerdere weekendjes weg houden we ook in stand (vandaar de naam), en dat blijven we hopelijk ook doen!

Tom, Erwin, Frank: de middelbare schoolvrienden. Inmiddels uitgewaaierd over heel het land. We zien elkaar soms lange tijd niet, maar áls wij elkaar zien, voelt die lange tijd als de dag van gisteren. Carnaval blijft elk jaar een mooie traditie, jullie ook bedankt mannen voor de nodige ontspanning. Tom, bedankt voor het prachtige ontwerp van dit boekje!

Mijn broer, Jelmer, ook jou wil ik ook bedanken. Het hoogtepunt van de afgelopen jaren was onze roadtrip door Scandinavië (jouw idee!). Het was een prachtige reis door Denemarken, Noorwegen en Zweden. Na een nacht had je denk ik wel al spijt, want ik snurkte de hele camping bij elkaar. Ook waren we niet al te best voorbereid, per slot van rekening gingen we in juni naar Noorwegen. Wisten wij veel dat we plots op onze sneakers en in spijkerbroek tot onze knieën in de sneeuw stonden. We hebben de afgelopen jaren heel wat tenniswedstrijden gespeeld met ons tennisteam. Jelmer, Peter, Glenn, Didier, Robin, Bryan, jullie ook bedankt, niet alleen voor de sportieve prestaties met als hoogtepunt ons kampioenschap in de eerste klasse in 2019, maar met name voor de gezelligheid en ontspanning.

Pap en Mam. Pap was altijd een beetje ongeduldig. Telkens als ik weer eens op bezoek kwam was hij in de veronderstelling dat mijn proefschrift al af was. Nu is het dan eindelijk echt af. Ook kon ik er altijd op rekenen dat er iets lekkers uit eigen keuken op tafel stond, hoe 
onverwacht ik ook binnenwipte. Zodra ik de gang instapte kon ik al ruiken, dankzij jouw geweldige kookkunsten mam, dat er weer gebakken of gebraden werd. Mijn persoonlijke favoriet natuurlijk je Limburgs zoervleesj. Bedankt voor jullie steun en bedankt dat jullie mij altijd de vrijheid hebben gegeven om mij te ontwikkelen zoals ik dat graag wilde. Zonder jullie was het niet gelukt. Opa's en oma, soms wisten jullie niet wat mijn onderzoek nou precies inhield. Nooit waren jullie daarom minder enthousiast, en wat waren jullie als koempels (opa Heinz 21 jaar op de Oranje Nassau III, opa Wiel 28 jaar op de Laura en Julia) trots dat jullie kleinzoon dokter werd. Opa, al 25 jaar gaan we naar alle wedstrijden van Roda JC, met hoogteen dieptepunten. Hopelijk mogen we binnenkort weer eens gaan. Tot die tijd zullen we ons vermaken met het leggen van een kaartje. Dank voor jullie steun en enthousiasme.

Lieve Lindsay, d'r letzte d'r betzte. Inmiddels ben je zelf ook al bijna klaar met je promotie. Samen promoveren kan leuk zijn, maar soms ook niet. Je weet precies van elkaar waar je tegen aanloopt. Een blik kan genoeg zijn om te weten dat de ander een dag vol tegenslagen heeft gehad. Altijd zorgde je er dan toch weer voor dat die tegenslagen snel weer vergeten werden. Niet in het minst omdat we aardig wat mooie reizen achter de rug hebben die ons onder andere langs Vietnam, Thailand, Cambodja en Amerika brachten. Hopelijk kunnen we snel weer eens samen op reis! Inmiddels hebben we een huis gekocht. Dat was nogal een bewogen periode, maar nu hebben we een prachtig plekje. Ik heb van veel mensen in dit dankwoord geschreven over hun belangrijke bijdrage, maar ondanks dat je niet (letterlijk) hebt bijgedragen aan het proefschrift was het zonder jouw steun écht niet gelukt. Lieve Lindsay, bedankt voor je liefde, vertrouwen en support. 


\section{About Jorne}

Jorne Ubachs was born on February $6^{\text {th }}, 1990$ in Landgraaf, the Netherlands. He attended secondary school at Eijkhagen college in Landgraaf. After completion of secondary school he went on to study Medicine at Maastricht University in Maastricht. He performed a clinical rotation in dermatology in Manipal, India, in 2014. His masters' thesis called "The influence of sarcopenia on survival and surgical complications in ovarian cancer patients undergoing primary debulking surgery" was supervised by professor Roy Kruitwagen. After completion of his senior clinical internship at the department of obstetrics and gynecology, supervised by dr. Brigitte Slangen, he went on to become a full-time

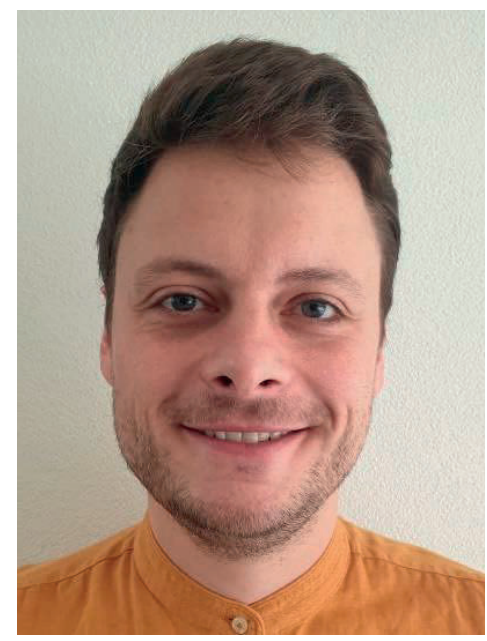
PhD-student. His thesis focuses on body composition alterations and homeostasis in ovarian cancer patients and their influence on outcome. The results of his research are published in this thesis. He was supervised by professor Roy Kruitwagen, professor Steven Olde Damink, professor Toon Van Gorp, and doctor Sander Rensen. In January 2020 Jorne became a resident in the department of obstetrics and gynecology at VieCuri hospital in Venlo and has been working there since. 


\section{List of publications (full papers)}

- Ubachs J, et.al., Ovarian cancer ascites induces skeletal muscle wasting in vitro and reflects sarcopenia in patients; revisions, Journal of cachexia, sarcopenia and muscle, 2021.

- Ubachs J, et.al., Gut microbiota and short chain fatty acid alterations in cachectic cancer patients; Journal of cachexia, sarcopenia and muscle, epub ahead of print 2021.

- Ubachs J, et.al., No influence of sarcopenia on survival of ovarian cancer patients in a prospective validation study. Gynecologic Oncology, 2020. 159(3), 706-711.

- Ubachs J., et.al., Sarcopenia and ovarian cancer survival: a systematic review and meta-analysis. Journal of cachexia, sarcopenia and muscle, 2019. 10(6), 1165-1174.

- Ubachs, J., et al., The influence of sarcopenia on survival and surgical complications in ovarian cancer patients undergoing primary debulking surgery. European journal of surgical oncology, 2017. 43(4): p. 717-724.

- Rutten, I.J.G., Ubachs. J. et al., Psoas muscle area is not representative of total skeletal muscle area in the assessment of sarcopenia in ovarian cancer. J Cachexia Sarcopenia Muscle, 2017. 8(4): p. 630-638.

- Ubachs, J,. et.al., Cachexie en veranderingen in lichaamssamenstelling zijn onderbelichte factoren bij de behandeling van kanker. Nederlands Tijdschrift voor Oncologie, 2018. 15 (5) 180-188.

\section{Conference abstracts}

- Jorne Ubachs, Ovarian cancer ascites-induced skeletal muscle cell wasting in vitro correlates to clinical muscle measures of the patient. Cancer cachexia conference, Montreal 2020. Abstract published in: Current Opinion in Supportive and Palliative Care.

- Jorne Ubachs et al; Is sarcopenia an independent predictive factor for survival in ovarian cancer? A validation study, Sarcopenia, Cachexia and Wasting Disorders conference 2019, Berlin.

- Jorne Ubachs, Koole S, et al. Loss of skeletal muscle mass during neo-adjuvant chemotherapy and the relation to survival in patients with ovarian cancer; a prospective analysis of the OVHIPEC-1 cohort International Journal of Gynecologic Cancer 2019;29:A532 
- Jorne Ubachs; 'Gut Microbiota Composition and Short-chain Fatty Acid Levels in Human Cancer Cachexia: A Pilot Study', International Medical Postgraduate Conference 2018, Hradec Kralove - Prague.

- Jorne Ubachs, Gut microbiota composition and short-chain fatty acid levels in human cancer cachexia: a pilot study; J Cachexia Sarcopenia Muscle. 2018 Dec; 9(6): 11211184.doi: 10.1002/jcsm.12365

- Jorne Ubachs, et al 'Sarcopenia negatively affects overall survival in ovarian cancer patients undergoing primary debulking surgery', 20th International Meeting of the European Society of Gynaecological Oncology (ESGO) 2017, Vienna.

- Iris J. Rutten, Jorne Ubachs, Psoas muscle measurements are inferior to total skeletal muscle measurements in the assessment of sarcopenia in ovarian cancer J Cachexia Sarcopenia Muscle. 2017 Feb; 8(1): 161-183. doi: 10.1002/jcsm.12182 


\section{List of abbreviations}

- Akt

- ALAT

- ASA

- ASAT

- AUC

- $\mathrm{BCA}$

- BIA

- BMI

- BRCA

- BSA

- cDNA

- $\mathrm{Cl}$

- CINAHL

- CMI

- CRP

- CRS

- CT

- CTCAE

- DEXA

- Df

- DLT

- DM

- DMEM

- DNA

- ELISA

- EPA

- ERK

- FBS

- FIGO

- FOXO

- $\mathrm{GCIG}$

- GDF-15

- GDF-8

- GGT

- $\mathrm{GI}$

- GM

- $\mathrm{Gr}$

- gRADE Evaluation

- $\mathrm{Hb}$ protein kinase $b$

alanine transaminase

American Society of Anesthesiologists

aspartate transaminase

area under the curve

bicinchoninic acid assay

body impedance analysis

body mass index

breast cancer susceptibility gene

body surface are

complementary DNA

confidence interval

Cumulative Index to Nursing and Allied Health Literature

core muscle index

c-reactive protein

cytoreductive surgery

computed tomography

Common Terminology Criteria for Adverse Events

dual-energy X-ray absorptiometry

degrees of freedom

dose limiting toxicity

differentiation medium

Dulbecco's modified Eagle's medium

deoxyribonucleic acid

enzyme linked immunosorbent assay

eicosapentaenoic acid

extracellular signal-regulated kinases

fetal bovine serum

International federation of obstetrics and gynecology

forkhead box protein

Gynecological Cancer InterGroup

growth differentiation factor 15

growth differentiation factor 8

gamma-glutamyltransferase

gastrointestinal

growth medium

grade

Grading of Recommendations Assessment, Development and

Hemoglobin 
- HBSS

- HDL

- HEPES

- HIPEC

- $\mathrm{HR}$

- $\mathrm{HU}$

- ICC

- iCRS

- ICU

- IDS

- IGF-1

- IL-1

- IL-1 $\beta$

- IL-6

- IL-8

- IMAT

- IV

- JAK

- L3

- LALSA

- LDL

- LIF

- LSC

- MA

- MAPK

- MCP-1

- MDRD

- MNA

- MRI

- mTORC

- MuRF-1

- $\mathrm{N}$

- $\mathrm{Na}$

- NACT

- NF-KB

- NPY

- NS

- OR

- OS

- OVHIPEC

- PA

- parp
Hanks' balanced salt solution

high density lipoprotein

4-(2-hydroxyethyl)-1-piperazineethanesulfonic acid

hyperthermic intraperitoneal chemotherapy

hazard ratio

Hounsfield unit

intraclass correlation coefficient

interval cytoreductive surgery

intensive care unit

interval debulking surgery

insulin-like growth factor 1

interleukin 1

interleukin 1 beta

interleukin 6

interleukin 8

intramuscular adipose tissue

inverse variance

janus kinase

third lumbar level

low activity liquid scintillation analyzer

low density lipoprotein

leukemia inhibitory factor

liquid scintillation cocktail

muscle attenuation

mitogen-activated protein kinase

monocyte chemoattractant protein 1

Modification of Diet in Renal Disease formula

mini nutritional assessment

magnetic resonance imaging

mechanistic target of rapamycin complex 1

Muscle RING-finger protein-1

number

not applicable

neoadjuvant chemotherapy

nuclear factor-kB

neuropeptide-Y

not significant

odds ratio

overall survival

ovarian hyperthermic intraperitoneal chemotherapy

psoas area

poly ADP ribose polymerase 
- $\mathrm{PBS}$

- PCA

- PDS

- PEDro

- PG-SGA

- PI3K

- PIF

- PLW

- PRIMS

- PRISMA

- qPCR

- QUIPS

- RECIST

- REDD-1

- RFS

- RNA

- ROC

- SAT

- SCFA

- SD

- SDS

- SE

- SM

- SMA

- SMI

- SMRA

- STAT3

- TNF- $\alpha$

- TSA

- UAC

- VAT

- WC

- WHO phosphate buffered saline

perchloric acid

primary debulking surgery

Physiotherapy Evidence Database

Scored Patient-Generated Subjective Global Assessment

phosphoinositide 3-kinase

proteolysis inducing factor

psoas length $x$ width

patient recorded integrated measurements

Transparent reporting of systematic reviews and meta-analyses

quantitative polymerase chain reaction

Quality In Prognosis Studies

Response evaluation criteria in solid tumors

regulated in development and DNA damage responses 1

recurrence free survival

ribonucleic acid

receiver operating characteristic

subcutaneous adipose tissue

short chain fatty acid

standard deviation

sodium dodecyl sulfate

standard error

skeletal muscle

skeletal muscle area

skeletal muscle index

skeletal muscle radiation attenuation

signal transducer and activator of transcription 3

tumor necrosis factor alpha

triceps skinfold assessment

upper arm circumference

visceral adipose tissue

wrist circumference

world health organization 


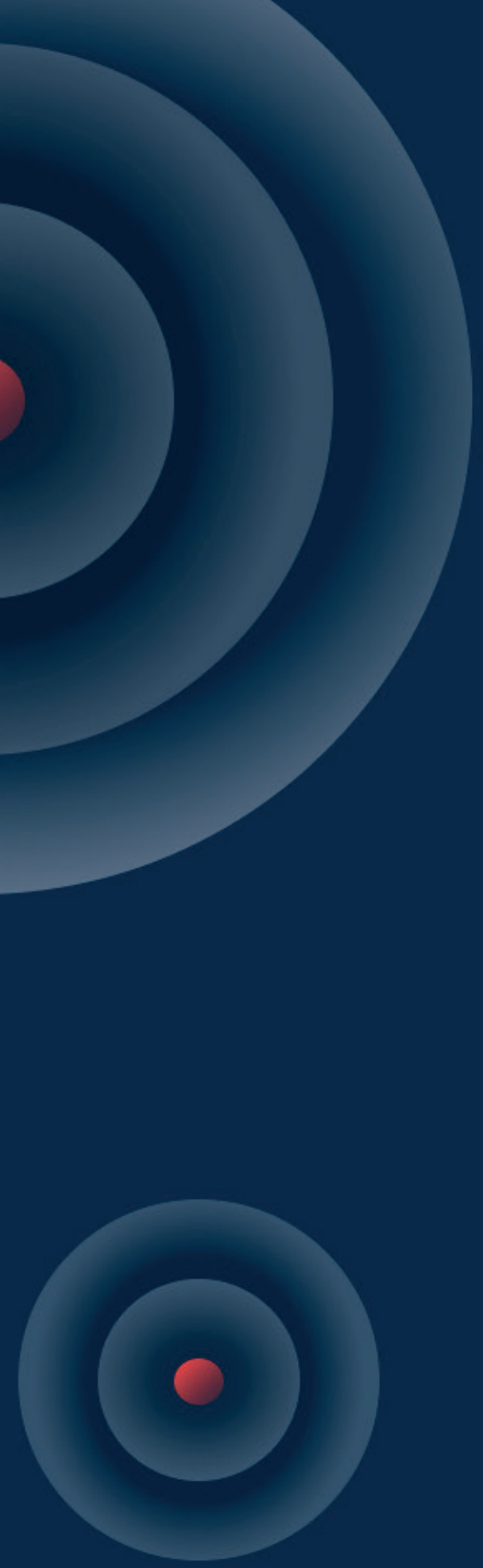